\title{
Lenidopterporum Calalogous
}

editus $a b$

\author{
Embrik Strand
}

Pars 61:

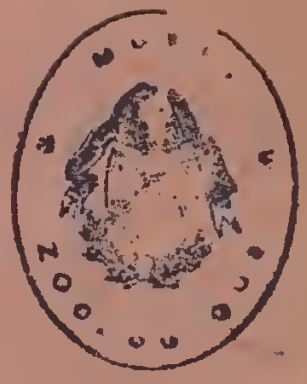

L. B. Prout

Geometridae:

Subfamilia Sterrhinae I.

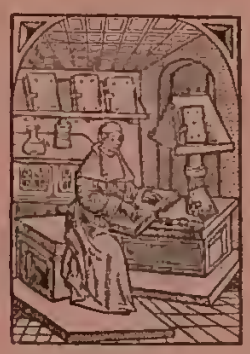

W. Junk

Berlin W. 15

22. III. 1934. 


\section{W. J U N K, Verlag und Antiquariat für Entomologie Berlin W. 15}

\section{Lepidopterorum Catalogus}

Pars 1: Ch. Anrivillius, Chrysopolomidae. 1911. 4 p.

2: \. Pagensterlier, Callislulidae. 1911. $14 \mathrm{p}$.

3: А. Pagenstecher, Libytheidac. 1911. $12 \mathrm{p}$.

4: H. Waguer ro R. Pfltzmer, Hepialidae. 1911. 26 p.

5: K. Strand, Noctuidae: Agaristinac. 1912. $82 \mathrm{p}$.

6: H. Meyrick, Adelidae, Mieropteregidae, Cracilariadae. 1912. 68 p.

7: 11. Zexny, Srutomidac. 1912. 179 p.

8: 1. B. Prout, Geometrilae: Brephinat, ()enocluminar. 1912. 91 p.

9: P. Mabille, Hesperilae: Sulf. Pyrthopgsinae. - MeDunnough, Megathpinidae. 1912. $22 \mathrm{p}$.

10: E. Meyrick, Tortricilate. 1912. 86 p.

11: H. Eltrintham of K. Jordan, Nymphatidae: Subfam. Acracinae. 1913. $65 \mathrm{p}$.

12, 18, 21, 23: II. Waguer, Ephingidae. 1913-1919. 420 p.

13: E. Meyrick, Camosinidae, Hcliodinidac, Glyphipterggidae. 1913. $53 \mathrm{p}$.

14: L. B. Prout, Genmetridae- Subfiun. Hemitheinae. 1913. 192 p.

15: K. W. von 1)alla Torre, (astuiidae: Subfam. Castniinae, Ycocastniinae. Pempligostolinae. 1913. $28 \mathrm{p}$.

16: E. Straud, Brahmacirlac. - H. G. Dyar et E. Strand: Megalop̧gidae, Daleeridae, Lpipyrop.jac. 1913. 35 p.

17: E. Jeyrick, Pterophoridat, Ormeodidae. 1913. $44 \mathrm{p}$.

19: E. Meyrick, II p̣onomeutidae, Plutellidae, Amphithoridne. 1914. $64 \mathrm{p}$.

20: K. W. von Dalla Torre, Thrstididae. 1914. $55 \mathrm{p}$.

22, 24, 26: E. Strand, Aretiidale [excl. Pericopinac]. 1919-1922. 900 p. - 45: F. Bryk, Perieupinate. 19:1. 5 T p.

25: h. W. rou lalla Torre, Cymatophordae. 1921. 38 p.

27: F. Bryk, Barouidae, Teinopalpidke, l'anassidae. 1923. 247 p.

28: K. W. vou Wnlla 'Turre el E. Strand, Lepidarbeliclae. - E. Strand, Heterogruididae. 1923. $14 \mathrm{p}$.

29: K. W. ron Halla 'Torre, Considae. 1923. $63 \mathrm{p}$.

30: K. W. ron Dalla Torre, Epiplemidae. Iraniiclae. 1924. $57 \mathrm{p}$.

31: K. W. von Malla Torre ef E. Straud, Aegeridae. 1925. 282 p.

32: 1R. ran Eeclic, (ochlidionidae (Limacoliclae). 1925. 81 p.

33: H. 13urgeli, Kyganiula I: Generis Zygaena palacaretica pars. 1926. $91 \mathrm{p}$.

34: K. W. vou Dalla 'Torre et E. Strand, Psychidae. 1929. 215 p. 


\title{
Octravo $8^{\circ}$ \\ ZMLA 612
}

\section{Lenidopterorum Cataloglus}

editus $a b$

\section{Embrik Strand}

\author{
Pars 61: \\ L. B. Prout
}

Geometridae:

Subfamilia Sterrhinae I.

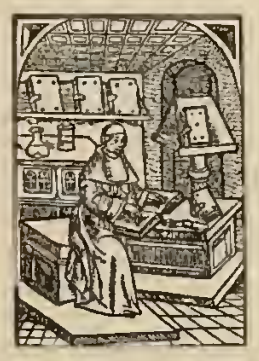

W. Junk

Berlin W. 15

1934.

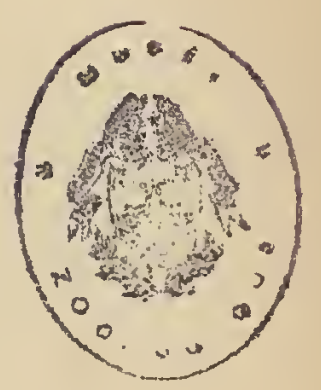



Zoologie. 


\title{
Fam. Geomefridae Subfam. Sferrhinae
}

\author{
Auct. L. B. Prouf
}

E rastriae Hb. (Tent., p. [2], ined.), Verz. Bek. Schmett., p. 299-301 (1825).

Cyclophorae Hb. (Tent., p. [2], ined.), Verz. Bek.

Schmett., p. 302, 303 (1825).

$S$ p h e coda e Hb. (Tent., p. [2], ined.), Verz. Bek. Schmett, p. 308-312 (1826).

Caberidi (part.) (Guen.) Dup., Cat. Méth. Lép. Eur., p. 268-271 (1845).

Acidalidi (Guen.) Dup., t. c., p. 271-277 (1845).

Fi idonidae (part.) Brd., Mém. Soc. Emul. Doubs, 2:2, p.137-140 (sep., p. 119-122) (1846).

Timandridi (part.) Steph., List Brit. Anim. Brit. Mus., 5, p. 226, $227(1850)$.

Dendrometrides (part.) H.-Sch., Syst. Bearb. Schmett. Eur., $3: 8$, p. 7 (1844); 3:18, p. 12-16 (1846); $3: 24$, p. $17-32$ (1847).

Dendrometridae (part.) II.-Sch., op. cit., pars 55, Syst. Lep. Eur., p. 28, 29 (1852) (Acidalin, Ephyra, Emmiltis). W. F. Kirby, Eur. Butt. \& Moths, p. $338-356$, t. 44, 47, 59 (1882). - Ch. Auriv., Nord. Fjär., p. 199-207 (1891).

Dendrometrina (part.) H.-Sch., Samml. Außereur. Schmett., 1, p. 25 (1856).

Ge o metrides sect. 2, Kayser, Deutschl. Schmett., p. 464479 (1857-58).

Ephyrida e Guen., Spec. Gén. Lép., 9, p. 402-421 (1858). - Walk., List Lep. Ins. Br. Mus., 22, p. 622-651 (1861); id., l. c., 26, p. 1573-1586 (1862). - Depuiset, Hist. Nat. Lóp. Eur. (in Sand, Monde des Pap.), p. 126 (1867).

Acidalidae Guen., t. c., p. 422-514; 10, p. $1-20$ (1858). - Walk., op. cit., 22, p. 651-755; id., 1. c., 23; p. $757-813$ (1861); id., l. c., 26, p. $1587-1617$ (1862); id., f. c., 35, p. 1618-1634, 1979 (1866). - Depuiset in Sand, 1. c., p. 126, 127 (1867). - F. O. Morris, Brit. Moths, 1, p. 148-171 (1872). - Meyr., Tr. N. Z. Inst., 16, p. 57 (1884). - Poppius, Acta Soc. F. F. Fenn., 
3 :3, p. 113-143 (1887). - V. Ström, Danm. Sommerf., p. 336-346 (1891) (Phytometridae a.).

E phyradae J. G. Morris, Cat. Lep. N. Amer. (Smithson. Inst. Misc. Coll., 3), p. 59 (1860).

Goniacidalinae (part.) Pack., Mon. Geom. U. S., p. 311 (1876).

A cidalinae Pack., t. c., p. 315-366 (1876). - Gmpbg.p Nova Acta Acad. Leop. Nat., $54: 5$, p. 479-544 (1890); id., 1. c., $58: 4$, p. $223-298$ (1892). - Strand, Archiv f. Naturg., 82 A : 3 , p. 33 (1916).

Zonosomatidae F. B. White, Scot. Nat., 3, p. 361 (1876).

I da eidae Butl., Pap. 1, p. 221 (1881). - Moore, Lop. Ceyl., 3, p. 447, 448 (1887). - W. F. Kirby, Handb. Lep., 5, p. 224-226 (1897); id., Butt. \& Moths Eur., p. 323 , t. $44,45,51$ (1903); id. in Hb. Zutr. (ed. Wytsman) (1912).

Ephyrina Plötz, Syst." Schmett. (Mitt. Nat. Ver. NeuVorpomm., 17), p. 3, 32 (1885).

A cidalina Plötz, l. c. (1885).

Timandrina Plötz, 1. c. (1885).

A cidaliadae Meyr., Tr. N. Z. Inst., 17, p. 63 (1885); id., Tr. Hnt. Suc. Lond., 1886, p. 203 (1886). - Turn.,

Tr. Roy. Soc. S. Austral., 46, p. 261-270 (1922).

Cyclophoridae Moore, Lep. Ceyl., 3, p. 441-446 (1887).

I d o e id a e Cotes \& Swink., Cat. Moths Ind., 4, p. 574 (1888).

- Hampson, Ill. Het., 8, p. 32 (1891).

Cyllopodidae (part.) W. F. Kirby, Syn. Cat. Lep. Het.,

1, p. 398-410 (1892); id., Handb. Lep., 3, p. 186 (1897).

Sterrhidae Meyr., Tr. Ent. Soc. Lond., 1892, p. 84-92 (1892). - Comstock, Man. Stud. Ins,, p. 273 (1895). (part.) H. Schultz, Deutsch. Ent. Zeits., 1914, p. 24 (1914). - Tillyard, Ins. Austral. \& N. Z., p. 451 (1926).

I do einae Warr., Proc. Zool. Soc. Lond., 1893, p. 358-362 (1893).

Cyllopodinae Warr., Nov. Zool., 2, p. 84 (1895). Prout, Tr. City Lond. Ent. Soc., 20, p. 23 (1911).

A cidaliinae Hampson, Faun. Ind., Moths, 3, p. 425-466 (1895). - Tutt, Brit. Moths, p. 239-247 (1896). - O. Nickerl, Spanner Böhmen, p. 3-11 (1907). - Reb., Berge's Schmetterlingsb. (ed. 9), p. 310-327 (1910). - Slevogt, Arb. Nat. Ver. Riga, (n. s.) 12, p. 167-172 (1910). Salay, Bul. Soc. Sti. Bucuresci, 19 :3, p. 506-520 (1910). - Prout, Tr. City Lond. Ent. Soc., 20, p. 21-32 (1911); id. in Seitz, Macrolep., 4, p. 34-152 (1913). - Galv. \& Preissecker, Jahresb. Wien. Int. Ver., 23, p. 63-80 (1913). Pience, Genit. Geom. Brit., p. 32-38 (1914). - Klöcker, Danm. Faun., 17, p. 9-24 (1915). - Forbes, Lep. New York, p. 24, 25 (1923). - Gaede, Tierwelt Deutsch., 14 (Schmett. 2: Nachtfalter), p. 227-240 (1929). - Romaniszyn, Faun. Motyli Polski, 1 (Praco Mon. Kom. Fizyog. Krakow, 6), p. 388-405 (1929). - Sterneck, Zeits. Oesterr. Ent.Ver., 17, p. 67 seq. (1932). - Hering, Tierwelt Mitteleur., Frgänz.-Bd. 1: Schmett., p. 320-332 (1932). - John \& Krizu, Latr. Noteic. Taurini, p. 120-124 (1932). 
Sterrhinae Warr., Nov. Zool,, 2, p. 90-101 (1895). Hulst, Tr. Amer. Ent. Soc., 23, p. 296-309 (1896). - Turn., Proc. Linn. Soc. N. Sth. Wales, $32: 4$, p. 635-700 (1908). - Prout, Nov. Zool., 23, p. 16 (1916). - Forbes, Journ. N. Y. Ent. Soc., 25, p. 47, 52 (1917); id., Psyche, 32, p. 112 (1925). - Lhomme, Amat. Pap., 3, p. 214 (1927). Derenne-Meyers, Faun. Belg., Géom., p. 10-20 (1929).

Ephyrinae Tutt, Brit. Moths, p. 247, 248 (1896).

A cidaliidae Bonhote \& N. C. Rothsch., Harrow Butt. \& Moths, 2, p. 18 (1897). - Aigner-Abafi, Magyar. Lepkéi, p. 91-96 (1907). - Furneaux, Butt. \& Moths, p. 275, 276, f. 183-185 (1911). - Brues \& Melander, Classif. Ins. (Bull. Mus. Comp. Zool. Harvard, 73), p. 210 (1932).

Monocteniinae Hulst, Tr. Amer. Ent. Soc., 23, p. 294, 295 (1896) (neo alior.).

Zonosomidae W. F. Kirby, Butt. \& Moths Eur., p. 322, t. 45 (1903).

Geometridae (part.) Lampert, Großschmett. u. Raup. Mitteleur., p. $216-224$, t. 65, 66 (1907). - Eckstein, Das Naturreich: Schmett., p. 222-224, t. 17, f. 2, 3 (1922).

Micropinidae W. F. Kirby in Hb. Zutr. (ed. Wytsman), p. 69 (1912).

Cyllopodicae Prout, Ann. Mag. Nat. Hist., (8) 6, p. 239 (1910).

A cidali icae Prout, Ann. Mag. Nat. Hist., (8) 6, p. 240 (1910).

Co symbi inae Prout, Tr. City Lond. Ent. Soc., 20, p. 23 (1911). - Pieroe, Genit. Geom. Brit., p. 35-38 (1914).

Ptychopodinae Pierce, Genit. Geom. Brit., p. 26-32 (1914).

Scop ulinae Hampson, Nov. Zool., 25, p. 384 (1918).

Acidali ini Handlirsch in Sehröder, Handb. Ent., $3: 15 / 16$, p. 929,930 (1924). - Janse, S. Afr. Journ. Sci., 22, p. 323, 339 (1925); id., Moths S. Afr., 1, p. 81, 92 (1932).

Scopuli ina Blackmoro, Check-List Macrolep. Brit. Col., p. $34,35(1927)$.

Kataloge: Steph., Cat. Brit. Ins., 2, p. 127, 128, 150-154 (1829); iḍ., List Brit. Anim. Brit. Mus., 5, p. 178-180, 218-223, 226, 227 (1850). - Curt., Guide Br. Ins., p. 165 (1830?); (ed. 2), p. 194 (1837). - Bsd., Gen. et Ind. Meth. Eur. Lop., p. 220-227 (1840). - H. Dbld., Syn. List Brit. Lop., p. 19, 20 (1849); id., Zool. Syn. List, (ed. 2), p. 6 (1859). - Heydenreich, Syst. Verz. Eur. Schmett., (ed. 3), p. 54, 55, 59, 60 (1851). - H.-Sch., Verz. Eur. Schmett. (Corr.-Bl. Zool.-Min. Ver. Regensb., 9), p. 29 (1855). - J. G. Morris, Cat. Lep. N. Amer., p. 59 (1860). - Stdgr., Cat. Lep. Eur. (ed. 1), p. 64-67 (1861); (ed. 2), p. 145-154 (1871). - Tengstr., Cat. Lop. Faun. Fenn. (Acta Soc. F. F. Fenn, 10), p. 25, 26 (1869). Knaggs, Cab. List Brit. Lep., p. 8 (1870). - Nillière, Mém. Soc. Sci. Nat. Cannes, 3, p. 186-201 (1873) (Alpes-Marit.). - Wllgr., Index Noct. et Geom. (Bih. Sv. Vet.-Ak. Handl. 2:4), p. 21, 22 (1874). - Sintenis, Neu. Verz. Estl. Schmett., p. 20, 21 (1876). - Porritt, List Yorks. Lop., p. 36-40 (1883). - South, Ent. Syn. List Brit. Lep., p. 13 (1884). - Cotes \& Swinh., Cat. Moths Ind., 4, p. 
529-533, 574-586 (1888). - Mina-Palumbo \& FaillaTedaldi, Nat. Sicil., 8:3, p. 58-61 (sep. p. 84-87) (1888) (Sizilien). - Hulst in J. B. Sm. et al., List. Lop. Bor. Amer., p. 68, 69 (1891). - Panis, Pap. France Cat. Méth, p. $194-196$ (1894). - Strand, Nyt Mag. f. Naturvid., 37, p. 46-56 (1899); id., I. c., 39 , p. $25-72$ (1901); id., 1. c., 40, p. $135-192$ (1902); id., l. c., 42, p. $109-179$ (1904). Strand, Archiv Math. Naturvid., 22, Nr. 5, p. 1-62 (1900). - Stdgr. \& Reb., Cat. Lep. Palaearct., 1, p. $265-279$ (1901). Kollmorgen, Iris, $13: 2$, p. 197-199 (1901) (Corsika). Kane, Cat. Lep. Ireland, p. 102-104 (1901). - Dyar, List N. Amer. Lep. (Bull. U. S. Nat. Mus., 52), p. 290-298 (1902). - Rothe, Verz. Schmett. Oesterreich-Ungarns (ed. 2), p. 34-36 (1902). - Bachmetjew, Hor. Soc. Ent. Ross., 35, p. 444-447 (1902) (Bulgarien). - J. B. Sm., Skinner \& Kearfott, Check List Lep. Bor. Amer., p. 70, 71 (1903). Spröngerts, Iris, $17: 2$, p. 187, 188 (1905) (Ostpyreneen). - Riodel, Iris, $18: 1$, p. 146-153 (1905) (Sachsen). Siepi, Cat. Lép. Bouches-du-Rhône (Ann. Mus. Marseille, Zoo!., 9), p. 102-105 (1905). - Matsumura, Cat. Lop. Japan, p. 119-124 (1905). - Barnes \& Mo Dunnough, Check List Iep. Bor. Aner., p. 101-103 (1917). - Janse, Check-List S. Afr. Lep. Het., p. $95-100$ (1917). - Strand, Archiv f. Naturg., 85 $\Lambda$ :4, p. 1-82 (1919). - Ch. Oberth., Et. Lép. Comp., $21: 2$, p. 19-30 (1923) '(Pyrénées-Orientales). - H. J. Turn., Ent. Rec, 37, supp., p. 3-5 (1927) (Britannien). - Stauder, Ent. Anz., 9, p. 70-72, 88-94, 115117, 131-136 (1929) (Fauna Ilyro-Adriatica).

Tabollen: Heinem., Tab. Bestimm. Schmett. Deutschl., p. 25, 26, 100-104 (1859). - Pack., Mon. Geom. U. S., p. 316 (1876). - Gmpbg., Syst. Geom. Zon. Temp. Sept., pars 4, p. 50, 51 (Nova Acta Acad. Lreop. Nat., 54, p. 482, 483) (1890). - V. Ström, Danm. Sommerf,, p. 337 (1891). - Meyr., Classif. Geom. Eur. Faun. (Tr. Ent. Soc. Lond., 1892), p. 85 (1892). - Hampson, Faun. Ind. Moths, 3, p. 425,426 (1895). - Hulst, Classif. Geom. N. Amer, (Tr. Amer. Eut, Soc, 23), p. 296, 297 (1896). - Ter Haar, Onze Vlind, p. 250 (1904). - Lameere, Man. Faun. Belg., 3, p. $704-708$ (1907) (Acidaliiens [sic]), - Turn., Rovis. Austral. Lep., IV (Proc. Linn. Soc. N. Sth. Wales $32: 4)$, p. 636-638 (1908). - D. Luc., Tabl. Det. Lép. France (Mise. Ent, 20, 21, supp.), p. 240-252, 313 (1913). Hoffmeyer \& Knudsen, Flora og Fauna, p. 133 (1931) (Acidalia spp., Bornholm Isl, Dania). - Hering, Tierwelt Mitteleur., Ergänz.-Bd. I: Schmett,, p. $320-330$ (1932) (Acidalia).

B i o log i e: Wilde, Pflanz. u. Raup. Deutschl., 2, p. 361-377 (1861). - Rouast, Ann. Soc. Linn. Lyon (n. s.), 29, p. 334-338 (1883). - R. Rössler, Raup. d. Grolschmett. Deutschl., p. 129-159, passim (Bestimmungstabellen) (1900). - Frionnet, Chenilles Macrolep. Franç. Géom., p. 11, 20, 48-222 (Bestimmungstabelleu), 227-232 (1904). - Blaschke, Raup. Eur., p. XII, XIII, 1-208 passim (1914).

I c on ographie: Berge, Schmetterlingsb. (ed. 2), t. 10, 11 (1851); (ed. 3), t. 47 (1863); (ed. 8), t. 43, 44 (1899). Praun, Abbild. Eur. Schmett., Geom., t. 4, 5 (1869). 


\section{Genus Rhodostrophia $\mathrm{Hb}$.}

Hb., Verz. Beik. Schmett., p. 300 (1825) (typus calabra Pet.). Walk., List Lrep. Ins., 23, p. 803 (1861). - Gmpbg., Nova Acta Acad. Leop. Nat. $49: 4$, p. 328 (1887); $58: 4$, p. 283 (1892). Meyr., Tr. Ent. Soc. Lond,, p. 92 (1892), - Swinh., Tr. Ent. Soc. Lond., 1894, p. 181 (1894). - Hampson, Faun. Ind. Moths, 3, p. 455 (1895). - Stdgr. \& Reb., Cat. Lep. Pal., 1, p. 278 (1901). - Reb. in Spulcr, Schmett. Eur. (Е. Hofm. Großschmett., orl. 3), 2, p. 28 (1904); id., Bergc's Schmetterlingsb. (ed. 9), p. 326 (1910). Prout in Seitz, Macrolop., 4, p. 35 (1913). - Eckstein, Schmett. Deutschl., 4, p. 14, t. 49, f. 6 (1923). - Hering, Tierwelt Mitteleur., Schmett., p. 332 (1932).

Pellon ia Dup., Hist. Nat. Lép. Eur., 7 : 2, p. 109 (1829); $8: 1$, p. 132 (1830); id., Cat. Méth., p. 243 (1845) (typus vibicaria Clerck). - Led., Verh. Zool.-Bot. Ver. Wien, 3, p .175, 195 (sep. p. 11, 31) (1853). - Ad. Spr., Deutsche Schmettkunde., p. 192 (1856). - H.-Sch., Samml. Außereur. Schmett., $1: 18 / 22$, p. 25 (1856). - Guen., Spec. Gén. Lép., 10, p. 6 (1858). - Heinem., Sclimett. Deutschl., 1, p. 707 (1859). - Stdgr., Cat. Lep. Eur., p. 67 (1861). - Constant, Cat. Lép. Saone-ct-Loire, p. 207 (1866). Snell., Vlind. Ned., Macrolep., p. 549 (1867). - Berce, Faun. Ent. Franç., Lép., 5, p. 183 (1873). - Fritsch, Denks. Akad. Wien, Math.-Nat. CI., 41 : 1, p. 94 (1879) (Phänologie). - Poppius, Acta Soc. F. F. Fenn., 3:3, p. 118 (1887). - Gmpbg., Nova Acta $\Lambda$ cad. Loop. Nat., $49: 4$, p. 330 (1887); 58:4, p. 280 (1892). - Ch. Auriv., Nord. Fjär., p. 205 (1891). - V. Ström, Danm. Somuncrf., p. 338 (1891). - Strgr., Tris, 5, p. 205 (1892) (Morphologie). Korb, Schmett. Mittel-Eur., p. 177 (1894). - Cholodkovsky, E. Hofm. Atlas Bab. Evr., p. 244, 245, t. 58 (1897).

I d a e a (part.) Children, Philos. Mag., (n. s.) 16, p. 464 (1839).

A spilates (part.) Bsd., Gen. et Ind. Meth., p. 187 (1840). - Ad. Spr., Isis, 1843, p. 264 (1843).

A spilades (part.) Heydenreich, Syst. Verz. Eur. Schmett. (ed. 2), p. 14 (1846).

A cidalia (part.) H.-Sch., Syst. Bearb. Schmett. Eur., $3: 18$, p. 13 (1846).

I déa (part.) Bertoloni, Nov. Comment. Bonon., 9, p. 149 (1849).

E u s a r c a (part.) H.-Sch., Syst. Bearb. Schmett. Eur., $3: 24$, p. 34 (1847).

E u s a r ca Led., Verh. Zool.-Bot. Ver. Wien, 3, p. 180, 235 (sep. p. 16, 71) (1853). - Gucn., Spec. Gén. Lép., 10, p. 143 (1858). - Walk., List Lop. Ins., 24, p. 1025 (1862). Gmpbg., Nova Acta $\Lambda$ cad. Leop. Nat., $49: 4$, p. 328 (1887); $65: 3$, p. 264 (1896). - Stdgr., Iris, 5, p. 206 (1892) (nec Chevrolat).

Pydn a H.-Sch., Samml. AuBereur. Schmett., 1, p. 47 (1856) (typus badiaria Freyer).

Deiocharis Butl., Proc. Zool. Soc. Lond., 1883, p. 172 (1883) (typus herbicolens Butl.).

Le p to sidia Hmpsn., Journ. Bomb. Soc. Nat. Hist., 14, p. 653 (1903) (typus anomala Warr.). 
jacularia Hb., Samml. Eur. Schmett., Geom., t. 84, f. 431 (1808-14); id., Syst.-Alph. Verz. Eur. Schmett., p. 44 (1822) (Eusarca dubia) [Südrußland]. - Eversm., Bull. Mose., $10: 6$, p. 55 (1837); id., Faun. Lep. Volg.-Ural., p. 373 (1844) (Asp.). - Bsd, Gen. et Ind. Meth. Eur. Lep., p. 188 (1840) (Asp.). Dup., Hist. Nat. Lép. Eur., supp. 4, p. 240, t. 71, f. 2 (1842) (Asp.). - H.-Sch., Syst. Bearb. Schmett. Eur., $3: 24$, p. 35 (1847); pars 31, t. 59, f. 365 (1848) (Eu.).: id., Samml. Außeneur. Sehmett., 1, p. 47 (1856) (Pyd.). - Heydenreich, Syst. Verz. Eur. Schmett. (ed. 3), p. 54 (1851) (Fidonia). - Guen., Spec. Gén. Lép., 10, p. 144 (1858) (Eu.). - Becker, Bull. Mosc., $31: 2$ (3), p. 165 (1858) (Asp.). - Walk., List Lep. Ins., 24, p. 1025 (1862) (Eu.). - Stdgr.; Cat. Lep. Eur, (ed. 2), 1, p. 175 (1871) (Eu.); (ed. 3), 1, p. 279 (1901). - Meyr., Tr. Ent. Soc. Lond., 1892, p. 92 (1892). Gmpbg., Nova Acta Acad. Ieop. Nat., 65, p. 266, t. 18, f. 236 (1896) (Eu.). - Reb. in Spuler, Schmett. Eur. (E. Hofm., GroBschmett., ed. 3) 2, p. 29, t. 71a, f. 26 (1904). - Prout in Seitz, Macrolep., 4, p. 36, t. 3e (1913). - Culot, Noct. \& Géom. Tur., 3, p. 99, t. 15, f. 295 (1918).

ab. carnosaria Stdgx., Iris, 12 , p. 345 (1900) (Eusarca).

var. minor Alph. in Romff., Móm. Lép., 6, p. 71 (1892) (Eu.). - Stdgr. \& Reb., Cat. Lep. Pal., 1, p. 279 (1901). - Prout in Seitz, Macrolep., 4, p. 36 (1913).

vastaria Christoph, Hor. Soc. Ent. Ross., 12, p. 261, t. 7 , f. 31,32 (1876) [Transeaspien]; id. in Romff., Mém. Lép., 2, p. 126, t. 6, f. 7 (1885) (Eu.). - Meyr., Tr. Ent. Soc. Lond., 1892 , p. 92 (1892). - Gmpbg., Nova Acta Acad. Loop. Nat., 65 : 3, p. 265 (1896) (Eu.). - Prout in Seitz, Macrolep., 4, p. 36 (1913).

badiaria Freyer, Neu. Beitr. Schmettk., $4: 59$, p. 115 , t. 354 ; f. 2 (1841) (Fidonia) (Sanepta). - H.-Sch., Syst. Bearb. Schmett. Eur., $3: 8$, t. 5 , f. 30 (1844) (non binom.). - Heydenreich, Syst. Verz. Eur. Schmett., (ed. 3) p. 54 (1851) (Hibernia). - Guen., Spec. Gén. Lép., 10, p. 144 (1858). (Eu.). Walk., List Lop. Ins., 24, p. 1026 (1862) (Eusarca). - Stdgr., Cat. Lep. Eur. (ed. 2), 1, p. 175 (1871) (Eu.); (ed. 3) 1, p. 279 (1901). - Romff., Mém. Lép., 2, p. 107 (1885) (Eu.). - Meyr., Tr. Ent. Soc. Lond., 1892, p. 92 (1892). - Gmpbg., Nova Acta Acad. Leop. Nat., $65: 3$, p. 265 (1896) (Eusarca). - Reb. in Spuler, Schmett. Eur. (E. Hofm., Großschmett., ed. 3), 2, p. 29, t. '65a, f. 10 (1904). - Stichel, Zeits. Wiss. Insbiol., 7 : 5/6, p. 162 (1911). - Prout

Südrußland, Thian-Schan, Mongolei

('Thian-Schan; Ordos

(Mongolei)

Transcaspien, Thian-Schan

Asiat

Türkei, Südrußland, ? Persien, Mesopotamien 
in Seitz, Macrolep., 4, p. 36 (1913). - Culot, Noct. \& Géom.' Eur., 3, p. 98, t. 15, f. 294 (1918). - Wehrli, Iris, $41: 1$, p. 52 (1927). e muc $i d a r i a$ Eversm., Bull. Mose., 10 :6, p. 58 (1837); id., Faun. Volg.-Ural., p. 388 (1844) (Fidonia) (err. det., neo. Hb.). prae canaria Eversm., Bull. Mose., 20:2, p. 81, t. 6, f. 4, 5 (1847) (Fidonia) (nom. nov.).

t e la ria H.-Sch., Syst. Bearb. Schmett. Eur., $3: 24$, p. 34 (1847) (Eu.); Samml. Außereur. Schmott., 1, p. 47 (1856) (Pyd.). Led., Verh. Zool.-Bot. Ver. Wien, 3, p. 180 (sep. p. .16) (1853) (Eu.). - Becker, Bull. Mosc., $31: 2$ (3), p. 163 (1858) (Ac.).

cervinaria (Redorsky, M. S.) H.-Sch., Syst. Bearb. Schmett. Eur., $3: 24$, p. 34 (1847) (Eu.).

pollenaria Freyer, Neu. Beitr., 7, p. 61 (1854) (Fidonia) (nom. nov. pro badiaria). praecisaria Prout in Seitz, Macrolep., 4, t. $3 \mathrm{~d}$ (non binom.) (1913) (nec Stdgr.).

terrestraria Led., Hor. Soc. Ent. Rass; 6, p. 90, Nord-Ostt. 5, f. 9 (1869) (Eu.). - Gmpbg., Nova Persien Acta Acad. Isop. Nat., 65:3, p. 265 (1896) (Eu.). - Stdgr., Cat. Lep. Eur., (ed. 2), 1, p. 175 (1871) (Eu.); (ed. 3), 1, p. 278 (1901). - Meyr., Tr. Ent. Soc. Lond., 1892, p. 92 (1892). - Prout in Seitz, Macrolep., 4, p. 37, t. $3 \mathrm{~d}$ (1913).

var. pellonaria (Stdgr., M. S.) Christoph in Romff., MǴm. Lép., 2; p. 126, t. 6, f. 6a, b (1885) (Eu.). - Stdgr., Cat. Lep. Pal,, 1, p. 278 (1901). - Prout in Seitz, Macrolep., 4 , p. 37 (1913). dispar Stdgr., Iris, 5, p. $205(1892)(E u$.$) (Zeraf-$
schan). - Meyr., Tr. Ent. Soc. Lond., 1892, p. 92 (1892). - Prout in Seitz, Macrolep., 4, p. 37 , t. $3 \mathrm{~d}(1913)$.

pudorata F., Ent. Syst., 3:2, p. 179 (1794) (Bar- Nord-Afrika, baria).

c a labraria H. Luc., Anim. Artic., 3, in Expl. Algiers, p. 389 (1849) (Asp.). Zell., Stett. Ent. Zeit., 15, p. 300 (1854) (Asp.) - G. Koch. Geogr. Verbr. Eur. Schmett., p. 118 (1854) (Asp.) '(part.). (err. det, nec calabra Petag.).

sicanaria (part.) Guen., Spec. Gén. Lép., 10, p. 10 (1885) (,nota") (Pell.). - Stdgr., Hor. Soc. Ent. Ross, 7, p. 156 (1870); id., Iris, 5, p. 291 (1892) (Pell.). - Zapater \& Korb, Mem. Soc. Esp. Hist. Nat., 21, p. 146 (1892) (Pellonia). - Ribbe, Lep.-Faun. Andal. (Iris 23, 3/4 Beih.), p. 312 (1912). Rothsch., Nov. Zool., 21, p. 342 (1914). Culot, Noct. \& Géom. Eur., 3, p. 97 (1918). - Ch. Oberth., Et. Lép. Comp., $19: 1$, p. 293 (1922).

(Unter-
Wolga)

(Wolga)

(sine loc.)

Transcaspien

West-

Turkestan

Spanien 
quadricalcarata Prout in Seitz, Macrolep., 4, p. 36, 38, t. 5 c (1913). - Zerny, Iris, 41 :2, p. 117 (1927); id., Eos 3, p. 397 (1927).

quadricalearata (err. typogr.) Rothsch., Bull. Soc. Maroc, 5 : 7/8, p. 341 (1926).

ab. perezaria Ch. Oberth., An. Soc. Esp. Hist. Nat., 4, p. 372, t. 17, f. 5, 6 (1875) (Pell.) (Carthagena). - Moyr., Tr. Ent. Soc. Lond., 1892, p. 92 (1892). - Gmpbg., Nova Acta Acad. Leop. Nat, $58: 4$, p. 282 (1892) (Pell.). - Strdgr., Cat. Lep. Pal. (ed. 3), 1, p. 278 (1901). - Prout in Seitz, Macrolep., 4, p. 38 (1913).

var. s i c a n a ria Zell., Stett. Ent. Zeit., 13, p. 180 (1852) (Geom.): id., Bull. Mose., $26: 2$ (3), p. 36 (1854) (Id. (Ac.)) (Sizilien). Lad., Verh. Zool-Bot. Ver. Wien, 3, p. 175 (1853) (Pell.). - H.-Sch., Syst. Bearb. Eur. Schmett., pars 63, t. 89, f. 548, 549 (1854) (non binom.); 6:68, p. 133 (1855) (Pell.). - Guen., Spec. Gén. Lép., 10, p. 10 (1858) (Pell.). - Bellier, Ann. Soc. Ent. Fr., (3) $8: 4$, p. 702 (1861) (Pell.). - Walk., List Lep. Ins., 23, p. 805 (1861). - Stdgr., Hor. Soc. Ent. Ross., 7, p. 156 (1870); id., Cat. Lep. Eur., (ed. 2), 1, p. 154 (1871) (Pell.j; (ed. 3) 1, p. 278 (1901). - Mina-Palumbo \& Failla-Tedaldi, Nat. Sicil., $8: 3$, p. 61 (1888) (Pell.). - Meyr., Tr. Ent. Soc. Iond., 1892, p. 92 (1892). - Gmpbg., Nova Acta Acad. Leop. Nat., 58 : 4, p. 281 (1892) (Pell.). - Rob. in Spuler, Schmett. Eur. (E. Hofm., Großschmett., ed. 3) 2, p. 29, t. 58, f. 29 (1904). - ? Torbrodt \& Müll.-Rutz, Sohmett. Schweiz, $2: 1$, p. 32 (1913). Prout in Seitz, Macrolep., 4, p. 38, t. $2 \mathrm{k}$ (1913). - Culot, Noct. \& Géom. Eur., 3, p. 97 , t. 14 , f. 290 (1918).

calabraria Hb.-Gey., Samml. Eur. Schmett., Geom.. t. 105, f. 547 (1828) (Geometra) (err. det., nec calabra Petag.).

cretacaria Ieb., Ann. Hofmus. Wien, 30, p. 135, f. Kreta 5 (1916) (sicanaria c.). - Caradja, Mem. Sti. Acad. Romana (3) $7: 8$, p. 28 (1931). calabra subsp. (s. n.) Prout in Seitz, Macrolep., 4, p. 38 (1913).

Morphologie: Caradja, 1. c. (1931).

Sieversi Christoph, Hor. Soc. Ent. Ross., 17, p. 115 (1882) (Aspilates) (Ordubad); id., Stett. Ent. Zeit., 53, p. 245 (1852) (Pell.). - Romff., Mém. Lép., 2, p. 93, t. 5, f. 5 (1885) (Pell.). Meyr., Tr. Ent. Soc. Lond., 1892, p. 92 (1892). - Stdgr., Cat. Lep. Pal., (ed. 3), 1, p. 278 (1901). - Prout in Seitz, Macrolep.,

Sizilien,

Süd-Italien 4, p. 39 , t. 2 k (1913).

Armenien, N.-W.Persien 
calabra Petag., Sp. Ins. Calabr., p. 38, t. 1, f. 32 (1787). - Led., Verh. Zool.-Bot. Ver. Wien, 3 , p. 259 (sep. p. 95) (1853) (Pell.). Wernb., Beitr. Schmettk., 2, p. 54 (1864) (Geometra). - Prout in Seitz, Macrolep., 4, p . 37, t. $2 \mathrm{k}$ (1913). - Zerny, Eos, 3, p. 397 (1927). - Reb. \& Zerny, Denks. Akad. Wien, Math.-Nat. Kl. 103, p. 103 (1931). irifasciata Cyr., Ent. Neap., p. 2, t. 1, f. 6 (1787) (nom. praeoce, nec. Scop.).

calabraria Hb., Beitr. Gesch. Schmett., $2: 4$, p. 9 , t. 2 , f. M (? 1791); id., Samml. Eur. Schmett., Geom., t. 10, f. 49 (1798), t. 70, f. $365(1800-08)$; id., Verz. Bek. Schmett., p. 300 (1825). - Bkh., Naturg. Eur. Schmett., 5, p. 500 (1794). - Esp., Schmett. in Abbild., 5, p. 33 , t. 4, f. 1,2 (1794). - Lep. de St. F. \& Serv. in Encycl. Méth. Hist. Nat., 10, p. 90 (1825) (Phalaena). - Tr., Schmett. Eur., 5:2, p. 446 (1825); $6: 2$, p. 262 (1828) (1d.). Meigen, Handb. Schmett., p. 222, t. 15, f. 2 (1827) (Geometra). - Dup., Hist. Nat. Lép. Eur., $8: 1$, p. 132 , t. 179 , f. 4, 5 (1830), p. [598] addenda (1831); id., Cat. Méth., p. 244 (1845) (Pell.). - Ménétr., Cat. Obj. Zool. Cauc., p. 267 (1832) (Phalaena). - Bsd., Gen. et Ind. Meth., p. 187 (1840) (Asp.).Brd., Móm. Soc. Emul. Doubs, 1, p. 20 (1841) (Asp.). - H.-Sch., Syst. Bearb. Schmett., Eur., $3: 18$, p. 14 (1846) (Ac.); $6: 68$, p. 133 (1855) (Pell.). - Zell., Isis, 1847, p. 505 (1847) (Id.); id., Stett. Int. Zeit., 13, p. 180 (1852) (Geometra). - O. Costa, Faun. Nap., Irep. Geom. p. 47, t. 4, f. 4 (1848) (Asp.). - Bertoloni, Hist. Lep. Bonon., p. 149 (1849) (Idèa). - Schläger, Stett. Ent. Zeit., 11, p. 37 (1850) (Idaea). - G. Koch, Geog. Verbr. Eur. Schmett., p. 118 (1854) (part.) (Asp.). - Guen., Speo. Gén. Lép., 10, p. 8 (1885) (Pell.). - Stdgr., Cat. Lep. Eur., p. 66 (1861); (ed. 2) 1, p. 154 (1871) (Pell.): (ed: 3) 1, p. 278 (1901). — Walk., List Lep. Ins., 23, p. 804 (1861). - Rössl., Jahrb. Nass. Ver. Nat., 19/20, p. 215 (1866) (Pell.). - Const., Cat. Lép. Saone-ot-Loire (Mém. Hist. Nat. Soc. Edu. 2) p. 207 (1866) (Pell.). - Millière, Mém. Soc. Cannes, 3, p. 201 (1873). - Berce, Faun. Ent. Franç., Lép., 5, p. 184, t. 51, f. 7 (1873). Cuni y Mart., Cat. Lep. Barcel., p. 135 (1874) (Pell.). - Curò, Bull. Soc. Ent. Ital., 10, p. 191 (1878) (Pell.). - A. Fuchs, Stett. Ent. Zeit., 39, p. 338 (1878); id., 1. c., 40, p. 40 (1879) (Pell.). - Sand, Cat. Íép. Berry et Auv., p. 89 (1879) (Pell.). - Kef., Betracht. Entwick. Schmett., p. 83 (1880) (Geometra). - Calberla, Iris 3, p. 61 (1890) (Pell.). - Gmpbg., Nova Áta

Süd-

Europa

bis

Ungarn,

Süd-Rußland 
Acad. Leop. Nat., $58: 4$, p. 280 (1892); id., l. c., 65, t. 15, f. 96 (1896) (Pell.). - Meyr., Tr. Ent. Soc. Lond., 1892, p. 92 (1892). - Caradja, Iris, 9, p. 69 (1896) (Pell.); Mem. Sti. Acad. Romana (3) $7: 8$, p. 28 (1931). - Reb., Ann. Hofmus. Wien, 18, p. 250 (1903); id. in Spuler's Schmett. Eur. (E. Hofm., Großschmett., ed. 3), 2, p. 29, t. 58, f. 30 (1904), id. in Berge's Schmetterlingsb. (ed. 9), p. 326, t. 40, f. 13 (1910). - Galv., Mitt. Nat. Ver. Univ. Wien, 7, p. 224 (1909). - Hafner, Ent. Zeits. (Frankf.), 24, p. 101 (1910). - Ribbe, Lep.-Faun. Andal. (Iris 23, Beil. 3/4), p. 312 (1912). Vorbrodt \& Müller-Rutz, Schmett. Schweiz, $2: 1$, p. 32 (1913). - Hoffmann \& Klos, Mitt. Nat. Ver. Steier., 53, p. 84, 98 (1917). - Culot, Noct. \& Géom. Eur., 3, p. 97, t. 14 , f. 291 (1918). - Heinrich, Beitr. Macrolep. Digne (Deutsch. Ent. Zeits. Beiheft), p. 99 (1923). - Dannehl, Ent. Zeits. (Frankf.), $40: 21$, p. 460 (1927). - Vorbrodt, Mitt. Schweiz. Ent. Ges., $14: 6$, p. 328 (1930).

rubrofasciata Dannehl, Ent. Zeits. (Frankf.), $47: 10$, p. 82 (1933).

ab. ta e n ia ria Freyer, Neu. Beitr. Schmettk., 2, p. 59, t. 131, f." 3 (Asp.). - Bsd., Gen. et Ind. Meth., p. 188 (1840) (Asp.). Dup., Cat. Mréth. Lép. Eur., p. 244 (1845) (Pell.). - H.-Sch., Syst. Bearb, Schmett., Eur., 3 : 18, p. 14 (1846) (Acidalia calabraria var.). - Led, Verh. Zool.-Bot. Ver. Wien 3, p. 175 (sep. p. 11) (1853) (Pell. calabra var.). - Prout in Seitz, Macrolep., 4, p. 38 (1913).

ab. 8 e parata Th.-Mieg, Le Nat., 11, p. 197 (1889). - Prout, Ent. Rec., 16, p. 287 (1904); id. in Seitz, Macrolep., 4, p. 38 (1913). - Culot, Noct. \& Géom. Eur., 3, p. 97 , t. 14, f. 292 (1918).

ab. 8 ang u ine a Th.-Mieg, 1. c. - Prout, 1. c. (1913).

ab. $m$ us cos a Bastelb., Ent. Zeits. (Stuttg.) 21, p. 217 (1908). - Prout, l. c. (1913).

ab. unilinea Prout in Seitz, Macrolep., 4, p. 38 (1913).

ab. languida Dannehl, Ent. Zeits. (Frankf.), $40: 21$, p. 460 (1927).

ab. violettaria Vorbrodt, Mitt. Schweiz. Ent. Ges., $12: 9 / 10$, p. 471 (1917); $14: 6$, p. 328 (1930).

ab. $p u n$ ct a ta Caradja, Mem. Sti. Acad. Romana (3) $7: 8$, p. 130 (1931). - tabidaria (part.), Stdgr., Cat. Iep. Eur. (ed. 2), 1, p. 154 (1871); (ed. 3) 1, p. 278 (1901). Ribbe, Lep.-Faun. Andal. (Iris 23, Beih. 3/4), p. 312 (1912) (et al. auct. pro parte) (nec

(Dalmatien)

(Drome)

(Pyrénées or.)

(Kreuznach)

(Spanien)

(Südtirol)

(Pte. Brolla)

(sine loc.) Zell.). 
var. c y priaria Reb., Jahresb. Wien. Ent. Cypern Ver., 26, p. 107 (1916) (sicanaria var.). cypria Prout, Nov. Zool., 27, p. 268 (1920) (calabra var.).

var. transcaucas ica Prout, Nov. Zool., 27, Transp. 268 (1920). - calabra Iod, Wien. Ent. caucasien Monats., 8, p. 169 (1864) (Pell.).

Morphologie: H.-Sch., op. cit., $3: 8$, Umrißtaf. Erkl., p. 10, t. 8, f. 20 (1841) (Ac.). - Zell., l. c. (1852). - Led., t. c., f. 8 (1853). - Guen., l. c., (1858). - Caradja, l. c. (1931).

Bi ol og ie: Bsd., Coll. Icon. Chen., Geom., to 2, f. 3,4 - Lod., t. c., p. 260 (sep. p. 96) (1853). - Mann, Verh. Zool.-Bot. Ver. Wien, 4, p. 563 (1854). - A. Fuchs, Stett. Ent. Zeit., 39, p. $333(1878), 40$, p. 40 (1880). - Rössl., Jahrb. Nass. Ver. Nat., 33/34, p. 168 (1881). - E. Hofm., Raup. Großschmett. Eur., 178, t. 40, f. 15 (1893).

tabidaria Zell., Isis, 1847, p. 506 (1847) (Id.); id., Stett. Fint. Zeit., 13, p. 182, 184 (1852) (Geometra). - Heydenreich, Syst. Verz. Eur. Schnett. (ed. 3), p. 59 (1851) (Pell.). - H.-Sch., Syst. Bearb. Schmett. Eur., $6: 55$, p. 64 (1852); pars 63 , t. 89 , f. $545-547$ (1854); pars 68, p. 133 (1855) (Pell.). Iod., Verh. Zool.-Bot. Ver. Wien, 3, p. 175, 259 (sep., p. 11, 95) (1853); 5, p. 211 (1855) (Pell. calabra var.). - Guen., Spec. Gén. Lép., 10, p. 9 (1858) (Pell.). - Bellier, Bull. Soc. Ent. Fr., (4) 1, p. 40 (1861). (Asp.). - Walk., List Lop. Ins., 23, p. 805 (1861). - Stdgr., Cat. Lep. Eur., p. 66 (1861); (ed. 2), 1, p. 154 (1871) (Pell.); (ed. 3) 1, p. 278 (1901); id., Hor. Soc. Ent. Ross., 7 , p. 156 (1870) (P. calabraria var.). - Kef., Betracht. Entwick. Schmett., p. 83 (1880) (Geometra). - Gmpbg., Nova Acta Acad. Leop. Nat, $58: 4$, p. 282 (1892) ( $P$. calabraria var.). - Caradja, Iris, 9, p. 69 (1896) ( $P$. calabraria var.). - Reb., Ann. Hofmus. Wien, 18, p. 250 (1903), 19, p. 256 (1904) (calabraria var.); id., Berge's Schmetterlingsb. (ed. 9), p. 326 (1910) (calabraria ab. (var.), part.). - Prout in Seitz, Macrolep., 4, p. 38 (1913) (calabraria var., ? sp.). - F. Wagn., Mitt. Münchn. Ent. Ges., 19, p. 180 (1929) (Rodostrophia calabraria var.). - Turati \& Fiori, Mem. Soc. Ent. Ital., 9 : 2, p. 207 (1930). - Caradja, Mem. Sti. Acad. Romana, (3) $7: 8$, p. 27 (1931). - Zerny, Iris, $47: 2$, p. 85 (1933). c a la bra ria Zell., Isis, 1847, p. 20 (1847) (Idaea) (nec calabra Petag.).

Süd-Ungarn,

Balkan-

länder,

Kleinasien, Syrien 
ab. su a vis Caradja, l. c., p. 28 (1931).

(Rumänien)

ab. ten uistrigat a Caradja, ibid. (1931).

Morphol ogie: Zell., I. c. (1852). - Guen.,

l. c. (1858). - Caradja, 1. c. (1931).

vibicaria Clerck, Ic. Ins. Rar., 1, t. 3, f. 2 (1759)

[Suecia]. - L., Faun. Suec. (ed. 2), p. 323 (1761); id., Syst. Nat. (ed. 12), $1: 2$, p. 859 (1767). - F., Syst. Ent., p. 623 (1775); id., Spec. Ins., 2, p. 246 (1781); id., Mant. Ins., 2, p. 187 (1787); id., Ent. Syst., $3: 2$, p. 139 (1794). - [Schiff.] Schmett. Wien., p. 117 (1775); (ed. IIl.), p. 478 (1801). Rott., Naturf., 11, p. 84 (1777). - Göze, Ent. Beytr., $3: 3$, p. 279 (1781). - De Villers, Linn. Ent., 2, p. 291 (1789). Scharfenberg in Scriba, Journ., 3, p. 230 (1791). - Brahm, Ins.-Kal., 2:1, p. 443 (1791). - Schwarz in Kleem., Beytr., $2: 2$, p. 173 , t. 22 , f. $1-8$ (1794). - Bkh., Naturg. Eur. Schmett., 5, p. 503 (1794). Esp., Sehmett. in Abbild., $5: 1$, p. 30, t. 3, f. 3-8 (1794). - Schrank, Faun. Boica, $2: 2$, p. 54 (1802). - Bchst., Getreue Abbild. Nat. Gegenst, 6, p. 78, t. 87 (1805). - Rossi, Faun. Etrusc. (ed. Tll.), 2, p. 304 (1808). - Haw., Lep. Brit., 2, p. 310 (1809) (Ceometra). - Tr., Schmett. Eur., $5: 2$, p. 446 (1825), 6:2, p. 263 (1828). (Id.). - Lep de St. F. \& Serv. in Encycl. Méth. Hist. Nat., 10, p. 90 (1825) (Phalaena). - Hb., Verz. Bek. Schmett., p. 300 (1825). - Dup., Hist. Nat. Lép., $7: 2$, p. 109 (1829), $8: 1$, p. 134, t. 179 , f. 6,7 (1830) (Pell.). - Steph., Cat. Brit. Ins., 2, p. 153 (1829) (Ptychopoda); id., III. Haust., 3, p. 207 (1831) (Pell.). - Curt., Guide Brit. Ins., p. 158 (1831) (Timandra). - Wd., Index Ent., p. 89, t. 20, f. 538 (1835) (Phalaena). - Ad. Spr., Isis 1839, p. 110 (1839) (Id.); 1843, p. 264 (1843) (Asp.). - Bsd., Gen. et Ind. Meth., p. 187 (1840) (Asp.). - Berge, Schmetterlingsb., p. 31, t. 2, f. 5 (1842) (Geometra). - Eversm., Faun. Iep. Volg.-Ural., p. 438 (1844) (Id.).

- Herold, Teutsch. Schmett., p. 45, t. 1, f. 13 (1844) (Geometra fibicaria). - H.-Sch., Syst. Bearb. Schmett. Eur., $3: 8$, Umrißtaf. Erkl., p. 10 (1841); $3: 18$, p. 13 (1846) (Acidalia). - O. Costa, Faun. Napoli, Lep. Geom., p. 44, t. 5, f. 3 (1848) (Asp.). Led., Verh. Zool.-Bot. Ver. Wien, 3, p. 175 (1853) (Pell.). - Guen., Spec. Gén. Lép., 10, p. 7 (1858) (Pell.). - Wernb., Stett. Ent. Zeit., 19, p. 284 (1858) [Geometra]. Heinem., Schmett. Deutschl., 1, p. 707 (1859) (Pell.). - Walk., List Lep. Ins., 23, p. 804 (1861). - Bellier, Ann. Soc. Ent. Fr., (3)

Europa u. NordKleinasien bis Sibirien 
$8: 4$, p. 702 (1861) (Pell.). - Peyrhff.,

Cat. Lép. Als., 1 (Bull. Soc. Colmar, 2), p. 103 (1862); (ed. 2), 1, p. 117 (1880) (Pell.). - Snell., Vlind. Ned., Macrolep., p. 550 (1867) (Pell.). - Stdgr., Cat. Iep. Eur. (ed. 2), 1, p. 154 (1871); id., Stett. Ent. Zeit., 43, p. 59 (1882) (Pell.).; id., Cat. Lep. Pal. (ed. 3), 1, p. 278 (1901). Beroe, Faun. Ent. Franç., Lép., 5, p. 183 (1873) (Pell.). - Zell., Stett. Ent. Zeit., 38, p. 453 (1877) (Pell.). - Poppius, Acta Soc. F. F. Fenn., 3:3, p. 118 (1887) (Pell.). De Graaf in Sepp, Ned. Tns. (ser. 2), 4, p. 197 , t. 35, 36, f. 15,16 (1887) (Pell.). Snell., ibid., p. 200 (1887) (Pell.). Calberla, Iris, 3, p. 61 (1890) (Pell.). - Ch. Auriv., Nord. Fjär., p. 205 (1891) (Pell.). - V. Ström, Danm. Sommerf., p. 338 (1891) (Pell.). - Gmpbg., Nova Acta Acad. Leop. Nat., $58: 4$, p. 284 (1892); 65:3, t. 15, f. 97 (1896). - Meyr., Tr. Ent. Soc. Lond., 1892, p. 92 (1892). Delahaye, Mém. Soc. Agric. Angers, 5 :2, p. 183 (1900). - Naufock, Jahresb. Wien. Ent. Ver., 12, p. 72 (1902). - W. Petersen, Lep. Estl., p. 116 (1902); (ed. 2), 1, p. 230 (1924). - Reb. in Spuler, Schmett. Eur. (E. Hofm., Großschmett., ed. 3), 2, p. 28 , t. 58, f. 28 (1904); id., Berge's Schmetterlingsb. (ed. 9), p. 326, t. 40, f. 12 (1910). - Hoyningen-Huene, Berl. Ent. Zeits., 49, p. 29 (1904). - Strand, Int. Ent Zeitschr., 3, p. 78 (1909). - Ribbe, Lep.Faun. Andal. (Iris 23, Beih. 3/4), p. 312 (1912): - Vorbrodt \& Müll.-Rutz, Schmett. Schweiz, $2: 1$, p. 32 (1913). - Prout in Seitz, Macrolep., 4, p. 39, t. $2 \mathrm{k}$ (1913). Hellweger, Jahresb. Vicent. Brixen, 39, p. 11 (1914). - Klöcker, Danm. Fauna, 17, p. 23, t. 2, f. 40 (1915). - Hoffmann \& Klos, Mitt. Nat. Ver. Steier., 53, p. 83 (1917). - Culot, Noct. \& Géom. Eur., 3, p. 96, t. 14, f. 287 (1918). - Strand, A rohiv f. Naturg., 85 A : 4, p. 5 (1919). - Stauder, Ent. Anz., 2, p. 81 (1922). - Zerkowitz, Beitr. Kennt. Lep. Ungarns (Buchbeil., Int. Ent. Zeits.), p. 46 (1927). - Reisser, Zeits. Oesterr. Ent.-Ver., 12, p. 106 (1927); id., Int. Ent. Zeits., $23: 42$, p. 180, f. 11 (1930) (ab., s. n.). - Sterneck, Prodr. Schmett. Böhm., p. 161 (1929). - Derenne-Meyers, Faun. Belg., G6́om., p. 10 (1929). Osthelder, Schmett. Südbayerns, $1: 3$, (Mitt. Münchn. Ent. Ges., 19, Beilage), p. 395 (1929). - Reb. \& Zerny, Denks. Akad. Wien, Math.-Nat. Kl., 103, p. 103 (1931). cruentata Scop., Ent. Carn., p. 226 (1763). a $r t r i$ os a [E. Geoffr.] Fourc., Ent. Paris, 2, p. 275 (1785). 
? cruentataria De Villers, Linn. Ent., 2, p. 323 (1789).

c a la braria Lep. de St. F. in Encycl. Méth., 10, p. 90 (1825) (Phalaena) (nec. Hb.).

(s. n.) .J. O. Sch., Icon. Ins. Ratisb., 1, t. 12 f. 5 (1767).

ab. $r u b r i f a s c i a t$ a Hufn., Bexl. Mag., 4, p. 612 (1767). - Prout in Seitz, Macrolep., 4, p. 39 (1913). - rubrociliata (Kühn, Naturf., 3, p. 7, t. 1, f. 3, 1774, s. n.) Göze, Ent. Beytr., 3:3, p. 387 (1781). - Jung, Alph. Verz. Schmott., 2, p. 176 (1792). vibicaria Hb., Samml. Eur. Schmett., Geom., t. 10, f. 50 (1798). - Guen., Spec. Gén. Lép., 10, p. 8 (1858) (var. A.). - fasciata Reb., Berge's Schmetterlingsb., (ed. 9), p. $326(1910)$.

ab. ro seata Erschoff, Hor. Soc. Ent. Ross., 22 , p. 201, t. 11, f. 2 (1888) (Pell.) (Irkutsk). - Snell. in Sepp, Ned. Ins., (2) 4, t. 36, f. 18 (1887) (s. n.). - Prout in Seitz, Macrolep., 4, p. 39 (1913).

ab, intermedia Kempny, Jahresb. Wien. Ent. Ver., 6, p. 63 (1896). - Prout, 1. c. Schawerda, Verh. Zool.-Bot. Ges. Wien, 70, p. (96) (1920).

ab. re ctilin e a ria Meves, Ent. Tidskr. (20, p. 221, 1899, s. n.), 35, p. 124 (1914).

ab. augustiniaria Fernandez, Eos, 7, p. 215, t. 2 , f. 7 (1931) (Rodostrophia vibicaria var.) (Sierra Nevada).

var. strig a ta Stdgr., Cat. Lep. Eur., (ed. 2), Süd-Europa,

1, p. 154 (1871) (Andalusien, Nordpersien);

1, p. 278 (1901). - Calberla, Iris, 3, p. 61 (1890). - Zapater \& Korb, Mem. Soc. Esp. Hist. Nat., 21, p. 146 (1892). - Caradje, Tris, $6: 2$, p. 230 (1894). - Alph. in Romff., Mém. Lép., 9, p. 50 (1897). - Ribbe, Lep.Faun. Andal. (Iris, 23, Beih. 3/4), p. 312 (1912). - Prout in Seitz, Macrolep., 4, p. 39, t. $2 \mathrm{k}$ (1913). - Vorbrodt \& Müll.Rutz, Schmett. Schweiz, 2:1, p. 32 (1913). - Culot, Noct. \& Géom. Eur., 3, p. 96, t. 14, f. 288 (1918). - Ch. Oberth., Et. Lép. Comp., $19: 1$, p. 293 (1922). - Zerny, Eos, 3, p. 398 (1927). - Rothsch., Nov. Zool., $38: 2$, p. 328 (1933). - vibicaria Schwarz in Kleem. Beytr., $2: 2$, t. 22, f. 8 (1794). - De Graaf \& Snell. in Sepp, Ned. Ins. (2) 4, t. 36, f. 17 (1887). - Rothsch., Nov. Zool., $38: 2$, p. 328 (1933).

var. unicolorata Stdgr., Cat. Iop. Eur. Ala-Tau, (ed. 2), 1. p. 154 (1871) (Altai); id., Iris, 5, Juldus, p. 207 (1892) (Pell.); id., Cat. Lep. Pal, (ed. Altai 3), 1, p. 278 (1901). - Prout in Seitz, Macrolep., 4, p. 39 (1913) (? sp. div.). Culot, Noct. et Géom. Eur., 3, p. 96, t. 14, f. 289 (1918).

(Berlin)

(Eisenach)

(sine loc.)

(Schneeberggebiet)

(Stockholm)

Nord-Afrika,

Persien,

Al.-Tau 
Morphologie: H.-Sch., Syst. Bearb. Schmett. Eur., 3:8, Umrißtaf., t. 8, f. 18, 19 (1844). - Hayek, Handb. Zool., 2 :2, p. p. 323, f. 1580 B (1880). - Snell. in Sepp, 1. c. (1187). - Peyron, K. Vet. Ak. Handl., $44: 1$, p. 208, t. 8, f. 20 (1909) (ovum).

B i olog i e: Réaumur, Mém. Hist. Ins,, 1, p. 104 , t. 1 , f. $14-16(1734)$; 2 , p. 385 , t. 28 , f. 18 (1736). - Brahm 1. c. (1791) - - Bkh., 1. c. (1794). - Schwarz, 1. c. (1794). Esp., 1. c. (1794). - Tisch., Taschenb. Schmett., p. 122 (1804). - Guen., l.c. (1858). - Wilde, Pflanz. u. Raup. Deutschl., 2, p. 377 (1861). - Assmuss, Stett. Fnt. Zeit., 24, p. 401 (1863). - Rössl., Jahrb. Nass. Ver. Nat., 33/34, p. 168 (1881). - De Graaf in Sepp, 1. c. (1887). - E. Hofm., Raup. Großschmett. Eur., p. 177, t. 40, f. 14 (1893). - De Rougemont, Bull. Soc. Neuchatel Sci. Nat., 31, p. 15 (1903). - Frionnet, Chen. Macrolep. Franç., p. 163 (1904). C. Schneider, Ent. Anz., 9:23, p. 443 (1929).

auctata Stdgr., Hor. Soc. Ent. Ross., 14, p. 442 (1878) (Pell.) (Kleinasien). - Romff., Mém. Lép., 2, p. 93, t. 5, f. 4 (1885) (Pell.). Meyr., Tr. Ent. Soc. Lond., 1892, p. 92 (1892). - Gmpbg., Nova Acta Acad. Leop. Nat, $58: 4$, p. 283 (1892). - Prout in Seitz, Macrolep., 4 , p. 39, t. 2 k (1913).

adauctata Stdgr., Iris, 5, p. 160 (1892) (Pell.) Zerafschan (Margelan). - Meyr., Tr. Ent. Soc. Iond., bis Ili 1892, p. 92 (1892). - Prout in Seitz, Macrolep., 4, p. 40, t. 3 d (1913) (sequ. subsp.?).

cinerascens Moore, Lep. Coll. Atk., p. 264 (1888) (Phyletis) (West-Kaschmir). - Hampson, Faun. Ind., Moths, 3, p. 458 (1893). - Prout in Seitz, Macrolep., 4, p. 40, t. 5 d (1913).

s u b fla vida Warr., Proc. Zool. Soc. Lond., 1893, p. 360 (1893) (Kashmix).

ab. rufilinea Prout in Seitz, 1. c. (1913) (s. 1.).

var. b o re a li s Swinh., Proc. Zool. Soc. Lond., Ostkaschmir, 1889 , p. 427 , t. 44, f. 8 (1889) (Phyletis) Punjab, (Kulu). - Hampson, Faun. Ind., Moths, 3, ? Sikkim p. 457 (1893). - bicolor rhoda Prout in Seitz, Macrolep., 4, p. 41, t. 7 a (1913) (err. det., nec bicolor Warr. 1895).

bicolor Warr., Nov. Zool., 2, p. 98 (1895) (Kukli, N.-W.[N.-W.-Indien]). - Hampson, Faun. Ind., Indien Moths, 4, p. 563 (1896) (inconspicua form. bicolor). - Prout in Seitz, Macrolep., 4, p. 40 (1913).

ab. $s u f f u s$ a Prout, t. c., p. 41 (1913). $\quad$ (N. W. India)

plesiochora Prout, Nov. Zool., 24, p. 306 (1917) (W. Westchina, China).

Tibet 
pelloniaria Leech, Ann. Mag. Nat. Hist. (6) 20, p. 108 (1897) (err. det., nec Guen.). meonaria (part.) Prout in Seitz, Macrolep. 4 , p. 40 (1913) (err. det., nec. Guen.).

monbeigi Oh. Oberth. Et. Lép. Comp., 20, p. 278, Westchina t. 561, f. 4829 (1921) (Phyletis).

meonaria Guen., Spec. Gén. Lóp., 10, p. 170 (1858) N.-W.(Phyletis) (Nord de l'Inde). - Walk., Iist Indien Lep. Ins., 24, p. 1053 (1862). - Prout in Seitz, Macrolep., 4, p. 40, t. 7 a (1913). Ch. Oberth., Et. Lép. Comp., 20, p. 276, 277, t. 561, f. 4826 (1921) (Phyletis).

pellaniaria Warr., Proc. Zool. Soc. Iond., 1888, p. 323 (1888) (Phyletis). - Hampson, Faun. Ind.' Moths, 3, p. 456, f. 205 (1895) (part.?) (err. det., nec Guen.).

grumarla Alph., Hor. Soc. Ent. Ross., $26: 3 / 4$, p. Kuku-Nor, 454 (1892) (Amdo); id. in Romff., Mém. Ĺ́p., Amdo 9, p. 50, t. 4, f. 1 (1897). (Pell.). - Stdgr., Cat. Iep. Pal. (ed. 3), 1, p. 278 (1901). Prout in Seitz, Macrolep., 4, p. 41 (1913).

glaucofusa Hampson, Journ. Bomb. Nat. Hist. Soc., Quetta $18: 1$, p. 46 (1907); $18: 2$, t. E, f. 40 (1908) (Dysethia). - Prout in Seitz, Macrolep., 4, p. 41, t. 5 d (1913).

poliaria Hampson, Journ. Bomb. Nat. Hist. Soc., Kaschmir $14: 4$, p. 653 (1903). - Prout in Seitz, Macrolep., 4, p. 41 (1913).

ab. roseat a Prout iu Seitz, 1. c. (1913).

anomala Warr., Nov. Zool., 2, p. 98 (1895) [Kasch- Kaschmir mir]. - Mampson, Journ. Bomb. Nat. Hist. Soc., $18: 1$, p. 52 (1907) (Lept.).

a r a e $a$ ria Hampson, Journ. Bomb. Nat. Hist. Soc., 14:4, p. 653 (1903); $18: 1$, p. 52 (1907) (aeariaar [sic!]) (Lept.).

praccisaria Stdgr., Iris, 5, p. 205 (1892) (Eusarca badiaria var.) (Zentralasien); id., Cat. Isep. Pal. (ed. 3), 1, p. 279 (1901) (badiaria var.). - Meyr., Tr. Ent. Soc. Lond,, 1892, p. 92 (1892). - Prout in Seitz, Macrolep., 4, p. 40 (1913).

ba di aria Christoph in Romff., Mém. Lép., 2, p. 126 (1885) (Eu.). - Prout, t. c., t. 3 (1913) (err. det., nec Frejer).

erythema Prout, Bull. Hill Mus., 1 : 3, p. 479 (1924) (? pr. Q⿱一一由. $f_{0}$ ).

Bokhara

Staudingeri Alph., Hor. Soc. Ent. Ross., 17, p. 186, Ferghana t. 8 , f. 82 (1882) (Eu.) (Kuldscha). Meyr., Tr. Fnt. Soc. Lond., 1892, p. 92 (1892). - Stdgr., Cat. Lep. Pal. (ed. 3), 1, p. 278 (1901). - Prout in Seitz, Macrolep,, 4, p. 41 , t. 3 d (1913).

Inconspicua Butl., Proc. Zool. Soc. Lond., 1886, p. N.-W.391 (1886) (Phyletis) (Murree); id., Ill. Het., Indien, 7, p. 22 (1889). - Warr., Proc. Zool. Soc. Lond., 1888, p. 323 (1888) (Phyletis). -

\author{
bis \\ Kuldscha \\ Afghanistan
}


Hampson, Faun. Ind., Moths, 3, p. 457 (1895). - Prout in Seitz, Macrolep., 4, p. 41 (1913).

var. $s u b c$ ons $p$ ic и a Prout, 1. c. (1913).

Afghanistan,

Chitral,

Kaschmir

cuprinaria Christoph, Hor. Soc. Ent. Ross., 12, p. 261, t. 7, f. 33 (1876) (Eusarca) (Nondpersien). - Romff., Mém. Lép., 2, p. 107, 126 , t. 6 , f. 8 a, b (1885) (Eu.). - Meyr., Tr. Ent. Soc. Lond., 1892, p. 92 (1892). -Gmpbg., Nova Acta Acad. Leop. Nat., 65 : 3, p. 264 (1896) (Eu.). - Stdgr. \& Reb., Cat. Lep. Pal. (od. 3), 1, p. 279 (1901). Prout in Seitz, Macrolep., 4, p. 42, t. 7 a (1913).

phaenicearia Hampson, Journ. Bomb. Nat. Hist. Soc., $18: 1$, p. 50 (1907); $18: 2$, t. E, f. 39 (1908) (Ac.).

cauquenensis Butl., Tr. Ent. Soc. Lond., 1882, 382 (1882) (Psamatodes) (sequ. form. ?).

dentiline a ta Warr., Nov. Zool., 2, p. 99 (1895).

Morphologie: Warr., 1. c.

Perruginaria Blanch. in Gay, Hist. Chile, 7sool., 7, Chile p. 96 , t. 7, f. 11 (1852) (Ac.) - Butl., Tr. Ent. Soc. Lond., 1882 , p. 381 , t. 16 , f. 7 (1882) (Psamatodes).

chilenaria Blanch, in Gay, Hist. Chile, Zool., 7, p. Chile 95 (1852) (Ac.). - Butl., Tr. Fint. Soc. Lond., 1882, p .382, t. 16, f. 6 (1882) (Psamatodes).

o b s cura Warr., Nov. Zool., 7, p. 164 (1900).

acidaria Stdgr., Iris, 5, p. 204 (1892) (Eu.) (Ferghana); id., Cat. Lep. Pal. (ed. 3), 1, p. 279 (1901). - Meyr., Tr. Ent. Soc. Lond., 1892, p. 92 (1892). - Prout in Seitz, Macrolep., 4 , p. 42 , t. 3 d (1913).

var. gris e aria Strgr., Iris, 5, p. 204 (1892); Transalai Cat. Lep. Pal. (ed. 3), 1, p. 279 (1901). Prout in Seitz, Macrolep., 4, p. 42, t. $3 \mathrm{~d}$ (1913).

var. a lexandraria Stdgr., Iris, 5, p. 205 (1892); id., Cat. Lep. Pal. (ed. 3), 1, p. 279 (1901), - Prout in Seitz, Macrolep., 4 , p. 42 (1913).

herbicolens Butl., Proc. Zool. Soc. Iond., 1883, p. Nord-Wiest172 (1883) (Del.) (Solun); 1886, p. 391 Himalaya (1886) (Phyletis); id., II. Het., 7, p. 22 (1889). - Hampson, Faun. Ind., Moths, 3, p. 457 (1895); id., Journ. Bomb. Nat. Hist. Soc., $12: 1$, p. 87 (1898). - Prout in Seitz, Macrolep., 4, p. 42 (1913).

muricolor Warr., Nov. Zool., 4, p. 61 (1897) (Sim- Kasauli, la). - Prout in Seitz, Macrolep., 4, p. 42 Simla, (1913) (herbicolens ab.) (pr. form. ?).

Kumaon 
subrufa Warr., Nov. Zool., 4, p. 224 (1897). - Simla Hampson, Journ. Bomb. Nat. Hist. Soc., $14: 4$, p. 653 (1903).

tristrigalis Butl., Ill. Het., 7 , p. 110 , t. 137, f. 1 Nord-West(1889) (Dharmsala). - Hampson, Faun. Ind., Indien, Moths, 3, p. 456 (part.) (1895). - Prout Bhutan in Seitz, Macrolep., 4, p. 42, t. 3e (1913).

peregrina Koll. in Hügel, Kaschmir, 4, p. 487 (1844) (Asp.) (Masuri).

Nord-West-

rara Butl., Ill. Het., 7, p. 111, t. 137, f. 2 (1889). - Hampson, Faun. Ind., Moths, 3, p. 456 (1895). - Prout in Seitz, 4, p. 42, t. $3 \in(1913)$.

olivacea Warr., Nov. Zool., 2, p. 99 (1895) (Dar- Sikkim jiling) (? pr. subsp.). Indien

(Dharmsala)

$14: 4$, p. 653 (1903).

haematozona Hampson, Faun. Ind., Moths 3, p. 457 Sikkim, (1895) (Sikkim).

Sikkim-

Tibet

pelloniaria Guen., Spec. Gén. Lép., 10, p. 169 (1858). (Phyletis) (Ind. or.). - Walk, List Lep. Ins., 24, p. 1053 (1862) (Phyletis). Swinh., Tr. Ent. Soc. Lond., 1894, p. 181 (1894); id., Cat. Lep. Het. Oxf. Mus., 2, p. 372 (1900). - Oh. Oberth., Itt. Lép. Comp., 20, p. 276, t. 560, f. 4825 (1923) (Phyletis).

th has iana Moore, Lep. Coll. Atk., p. 264 (1888) (Phyletis). - Swinh., Tr. Ent. Soc. Lond., 1894, p. 182 (1894).

meonaria Hampson, Faun. Ind., Moths, 3, p. 456 (1895) (err. det., nec Guen.).

yunnanaria $\mathrm{Ch}$. Oberth., Et. Lép. Comp., 20, p. 277, Chinat. 561, f. 4827 (1923) (Phyletis) (Tsekou). Tibet-

stigmalica Butl., TII. Het., 7, p. 110, t. 136, f. Nord-West19, 20 (1889) (Dharmsala). - Prout in Seitz, Macrolep., 4, p. 43, t. 3 e (1913) (vinacearia ab. stigmatica).

vinacearia (part.) Hampson, Faun. Ind. Moths, 3, p. 455 (1895).

similala Moore, Lep. Coll. Atk., p. 264 (1888) Sikkim (Phyletis) (Khasis). - Swinh., Tr. Ent. Soc. (var.?), Lond., 1894, p. 182 (1894). - Hampson, Faun. Ind., Moths, 3, p. 457 (1895). Prout, Journ. Bomb. Nat. Hist. Soc., $31: 1$, p. 135 (1926).

P stigmatic a Swinh., Tr. Ent. Soc. Lond., 1894, p. 181 (1894) (nec Butl.).

P vinacearia Swinh., Cat. Lep. Het. Oxf. Mus., 2, p. 272 (1900) (nec Moore).
Kumaon,

Assam

Indien

Khasiberge,

Birma 
vinacearia Moore, Proc, 7ool. Soc. Lond., 1867, p. Sikkim, 642 (1867) (Anisodes?) (Bengal). - Hamp- ? Tibet son, Faun. Ind., Moths, 3, p. 455 (1895) (var.?) (part.). - Prout in Seitz, Macrolep., 4, p. 43 (1913).

bisinuata Warr., Nov. Zool., 2, p. 98 (1895) ㅇ Zentral("Japan" [Westchina]). - Prout in Seitz, Macrolep., 4, p. 43 (1913); id., Nov. Zool., 24, p. 306 (1917).

vinacearia Leech, Ann. Mag. Nat. Hist., (6) 20, p. 108 (1897) (err. det., nec Moore). s $i$ ne $n$ s $i$ s Prout in Seitz, Macrolep., 4, p. 43 ơ (vinacearia var.) (1913).

China

bis

Yunnan

u. Tibet,

Formosa

(Moupin)

tremiscens Prout, Nov, Zool., 25, p. 77 (1918).

SüdI.

Szechuan

philolaches Ch. Oberth., Et. Ent., 15, p. 22, t. 3, Süd-Westf. 26 (1891) (Onophos). - Leech, Ann. Mag. China Nat. Hist., (6) 20, p. 108 (1897). - Stdgr., Cat. Lop. Pal. (ed. 3), 1, p. 279 (1901). Prout in Seitz, Macrolep., 4, p. 43 (1913): Sterneck, Iris, 41 : 2, p. 152 (1927).

farinosa Q Warr., Nov. Zool., 4, p. 394 (1897).

var. tibetaria Stdgr., Tris, 8:2, p. 331 (1896) (Eusarca) (Kuku-Nor); Cat. Lep. Pal. (od. 3), 1, p. 279 (1901). - Prout in Seitz, Macrolep., 4, p, 43 (1913). - farinosa o' Warr., Nov. Zool., 4, p. 394 (1897) 44 (Kuku-Nor).

\section{Genus Apostates Warr.}

Warr., Nov. Zool., 4, p. 214 (1897). - Prout in Seitz, Macrolep; 4, p. 43 (1913).

solitaria Christoph in Romff., Mém. Lép., 3, p. 101, Transcaspien, t. 5, f. 4 (1887) (Fidonia?) (Transcaspien). Bokhara, - Stdgr., Cat. Lop. Pal. (ed. 3), 1, p. 278 Ili (1901) (Rhodostrophia?). - Prout in Seitz, Macrolep., 4, p. 44, t. 7a (1913). albiclathrata Warr., Nov. Zool., 4, p. 1 214 (1897) (Merv).

\section{Genus Tanaofrichia Warr.}

Warr., Proc. Zool. Soc. Lond., 1893, p. 360 (1893) (typus trilineata Warr.). - Prout in Seitz, Macrolep., 4, p. 44 (1913); id., Nov. Zoold, 22 , p. 324 (1915). 
prasonaria Swinh., Trans. Ent. Soc. Lond., 1892, Khasiberge p. 12 (1892) (Pligletis); id., l. c., 1894, p. 181 (1894) (Rhodostrophia). - Prout in Seitz, Macrolep., 4, p. 44 (1913).

pras o narius Hampson, Faun. Ind., Moths, 3, p. 455 (1895) (Erythrolophus). - Swinh., Cat. Iop. Het. Oxf. Mus., 2, p. 372 (1900) (Erythrolophus).

var. trilineata Warr., Proc. Zool. Soc. Sikkim, Lond., 1893, p. 361, t. $32, \quad$ f. 2 (1893) Nord-West(Sikkim); id., Nov. Zool., 2, p. 98 (1895) Indien [Rhodostrophia].

curvata Warr., Nov. Zool., 2, p. 98 (1895) (Rhodo- Bhutan strophia). - Prout, Nov. Zool., 22, p. 324 (1915).

orieutis Prout in Seitz, Macrolep., 4, p. 44, t. 7 a West(1913) (Chetu). - Sterneck, Iris, $41: 2$, p. China $152(1927)$.

trilineata Leech, Ann. Mag. Nat. Hist., (6) 20, p. 108 (1897) (nec Warr.).

bisinuata Prout, Nov. Zool., 22, p. 324 3 (1915) (nec Warr.).

\section{Genus Discoglypha Warr.}

Warr., Nov. Zool., 3, p. 110 (1896) (typus aureifloris Warr.). Prout, Nov. Zool., 22, p. 324 (1915).

Triorisma Warr., Nov. Zool., 4, (p. 226, indescr.) p. 395 (1897) (typus genuflexus Hampson).

Erythrolophus (part.) Hampson, Journ. Bomb. Nat. IHist. Soc., $12: 1$, p. 86 (1898).

genuflexus Hampson, Faun. Ind., Moths, 3, p. 453 Sikkim, (1895) (Erythrolophus) (Sikkim). - Warr., Khasiberge Nov. Zool., 4, p. 395 (1897) (Triorisma).

Hampsoni Swinh., Tr. Ent. Soc. Lond., 1892, p. Khasiberge, 14 (1892) (part.) (Idaea) (Khasiberge); id., Coylon l. c., 1894 , p. 179 (1894) (Dithalma); id., Cat. Lep. Het., Oxf. Mus., 2, p. 371 (1900). - Hampson, Faun. Ind., Moths, 3, p. 453 (1895) (Er.). - Warr., Nov. Zool., 3, p. $111(1896)$.

punctimargo Hampson, Faun. Ind., Moths, 3, p. Sikkim 453 (1895) (Er.) (Sikkim). - Warr., Nov. bis Zool., 3, p. 111 (1896).

Assam

sanguinata Warr., Nov. Zool., 3, p. 116 (1896) /Or- Khasiberge ganopoda).

sang u inatus Hampson, Journ. Bomb. Nat.

Hist. Soc., $12: 1$, p. 87 (1898) (Er.).

variostigma Warr., Nov. Zool., 3, p. 311 (1896). Khasiberge plenorbis Warr., MS. in Mus. Tring.

inflammata Warr., Nov. Zool., 3, p. 111 (1896). Khasiberge infl a m matus Hampson, Journ. Bomb. Nat. Hist. Soc., $12: 1$, p. 87 (1898) (Er.).

locupletata Prout, Nov. Zool., 24, p. 307 (1917). Khasiberge 
aureifloris Warr., Nov. Zool., 3, p. 111 (1896). - Khasiberge Hampson, t. c., p. 86 (1898) (Er.). var. parvifloris Prout, Nov. Zool., 24, p. Sikkim 306 (1917).

\section{Genus Organopoda Hampson}

Hampson, IIl. Het., 9, p. 147 (1893); id., Faun. Ind., Moths, 3, p. $4 \overline{5} 1$ (1895) (typus carnearia Walk.).

atrisparsaria Wehrli, Iris, 37, p. 62, t. 1, f. 10, 21 China

(1924) (Ostchina).

brunnearia Ch. Oberth., Et. Lép. Comp.,

12, p. 156 , t. 395 , f. 3356 (1916) (Ephyra)

(err. det., nec Leech).

brevipalpis Prout, Journ. Bomb. Nat. Hist. Soc., Ober-

$31: 1$, p. 136, $31: 2$, t. 1, f. 7 (1926). Birma

annulifera Butl., Ill. Het., 7, p. 107, t. 136, f. 10 Ceylon,

(1889) (Anisodes) (Dharmsala). - Hamp- Nord-West-

son, Faun. Ind., Moths, 3, p. 452 (1895). Indien,

Sikkim

carncaria Walk., List Lep. Ins., 22, p. 644 (1861) Ceylon,

(Anisodes) (Ceylon). - Moore, Lep. Ceyl., Nord-Indien,

3, p. 444 (1857) (Anis.). - ? A. Pagenst., Liu-Kiu u.

Jahrb. Nass. Ver. Nat., 41, p. 171 (1888) Formosa,

(Anis.). - Hampson, Ill. Het., 9, p. 148 Sumatra,

(1893); Faun. Ind., Moths, 3, p. 451, f. bis

204 (1895). - Swinh., Tr. Ent. Soc. Lond., Mentawi-

1894, p. 180 (1894). (Erithrolophrs); id., Inseln,

Cat. Lep. Het. Oxf. Mus, 2, p. 370 (1900). Luzon,

- Prout, Bull. Hill Mus., 4:1, p. 128 ?Amboina (1930).

? carnaria Hagen in Maass, Bei Liobensw.

Wilden, p. 210 (1902) (Anis.).

subbrunnca Warr., Nov. Zool., 4, p. 393 (1897) (S. Süd-

Celebes) (pr. var.?).

Celebes,

Sumbawa

(var.?)

olivescens Warr., Nov. Zool., 3, p. 374 (1896) Queensland, (Queensland). - Turn., Proc. Linn. Soc. Luisiaden, N. S. Wales, $32: 4$, p. 683 (1908). Bismarek-

Archipel,

? Luzon

var. orbic ulat a Prout, Nov. Zool., 27, p. Goliathberg 268 (1920).

(Holl.-Neuguinea)

orbata Warr., Nov. Zool., 14, p. 142 (1907).

Brit.-Neuguinea

cnecosticta Prout, Journ. F. M. S. Mus., $17: 1$, p. Kinabalu 


\section{Genus Mefallaxis Prout}

Prout, Nov. Zool., $37: 2$, p. 229 (1932) (typus semiustus Swinh.); id. in Seitz, Macrolep., 16, p. 49 (1833).

seminstus Swinh., Ann. Mag. Nat. Hist., (6) 14, Khasiberge p. 433 (1894) (Erithrolophus). - Hampson, Faun. Ind., Moths, 3, p. 452 (1895) (Erythrolophus). - Prout, Tr. Ent. Soc. Lond., 1910, p. 221 (1910) ("Erythrolophus"). Strand, Archiv f. Naturg., $82 \mathrm{~A}: 3$, p. 33 (1916) (Erythrolophus). - Prout, Nov. Zool., $37: 2$, p. 229 (1932).

semipurpurascens Hampson, Faun. Ind., Moths, 4, Sikkim, p. 563 (1896) (Rhodostrophia) (Bhutan). - Bhutan, Prout, 1. c. (1932). Malayische Halbinsol (var.?)

Icledlapa Prout, 1. c. (1932); id. in Seitz, Macrolep, Madagaskar 3 16 , p. 49 , t. 6 a (1933)

\section{Genus Pseuderythrolophus Prout}

Prout, Nov. Zool., 37 : 2, p. 229 (1932).

bipunctalus Warr., Nov. Zool., 6, p. 334 (1899) Neu-Guinea, (Erythrolophus) (Brit. New Guinea). - DampierProut, Ann. Mag. Nat. Hist., (10) 6, p. 690 Insel (1930) (Erythrolophus); id., Nov. Zool., $37: 2$, p. 229 (1932).

ge minipuncta Warr. MS. in Mus. Tring (Organopoda).

var. idmon Prout, Ann. Mag. Nat. Hist., (10) Fidji 1

6, p. 690 (1930).

\section{Genus Apostegania Prout}

Prout, Nov. Zool., $37: 2$, p. 230 (1932) (typus crina Swinh.).

reetilineata Swinh., Ann. Mag. Nat. Hist., (7) 17, Sumatra, p. 554 (1906) (Ptochophyle) (W. Sumatra). Sarawak - Prout, Nov. Zool., $37: 2$, p. 230 (1932).

crina Swinh., Tr. Ent. Soc. Lond., 1892, p. 14, t. Khasiberge, 1, f. 14 (1892) (Stegania) (Khasia Hills); Schan-Staa t. id., l. c., 1894, p. 179 (1894) (Dithalma). Cochinchina

(1895) (Stegania). - Prout, 1. c. (1932). 


\section{Genus Zalissolepis Warr.}

Warr., Nov. Zool., 2, p. 100 (1895).

subviolaria Guen., Spec. Gén. Lép., 9, p. 386 (1858) Cayenne (Amaurinia) (Cayenne). - Warr., Nov. bỉs SüdZool., 2, p. 100, 101 (1895). - Ch. Oberth. Brasilien Et. Lép. Comp., 1.2, p. 94, t. 390 , f. 3298 1 (1916) (Amaurinia).

\section{Genus Difhecodes Warr.}

Warr., Nov. Zool., 7, p. 102 (1900, Mar.) (typus erasa Warr.). Prout, Gen. Ins., 129, p. 249 (1912); id. in Seitz, Macrolep., 4, p. 46 (1913); 16, p. 49 (1933); id., Nov. Zool., 22, p. 324 (1915).

Neosterrha Warr., Nov. Zool., 7, p. 158 (1900, Aug.) (subgen.?) (typus thetis Warr.).

Mnesithetis (Warr., Nov. Zool., 4, p. 396, 6, p. 295, indeser.). - Swinh., Cat. Lep. Het. Oxf. Mus., 2, p. 370 (1900, Sep.) (typus idaea Swinh.).

Euthysana Warr., Nov. Zool., 14, p. 141 (1907) (typus phaenomeris Prout).

Dithecodes subg. Pseudacidalia Sterneck, Iris, $41: 2$, p. 154 (1927) (nom. praeocc., nec Pseudacidalia Hampson 1894).

Morphologie: Prout, 1. c. (1913); id., Nov. Zool., 25, p. 78 (1918); id., Mém. Soc. Zool. Fr., 29:5; p. 394 (1932).

pseudaeidalia Sterneck, Iris, $41: 2$, p. 152 (1927) West-

(subg. Pseudacidalia); $42: 2$, p. 137 (1928) China (pseudoacidalia, err. typogr.).

erasa Warr., Nov. Zool., 7, p. 102 (1900). - Prout Japan in Seitz, Macrolep., 4, p. 46 (1913); id., Nov. Zool., $35: 4$, p. 295 (1930).

v a с и a Swinh., Tr. Ent. Soc. Lond., 1902, p. 671 (1902) (Hemithea). - Prout, l. c. (1913).

ornithospila Prout, Entom., 44, p. 292 (1911) (Südkamerun); id., Mém. Soc. Zool. Fr., $29: 5$, p. 393 (1932); id. in Seitz, Macrolep., 16, p. 49 , t. 6 a (1933).

Principe,

Fr.-Guinea

bis

Kamerun,

Abyssinien

bis

Nyassa

delieata Warr., Nov. Zool., 6, p. 295 (1899) (Nn.) Uganda, (Masindi). - Prout, Mém. Soc. Zool. Fr., Kikuyu, $29: 5$, p. 394 (1932); id. in Seitz, Macro- ? Nigeria lep., 16, p. 49 (1933).

var. (?) brunne ifrons Hampson, Tr. Zool. Ruwenzori Soc. Lond., $19: 2$, p. 126, t. 4, f. 59 (1909) (Nemoria). - Prout, Gen. Ins., 129, p. 175 (1912) (Nemoria); id., Mém. Soc. Zool. Fr., 29 : 5, p. 394 (1932); id. in Seitz, Macrolep., l. c., t. 6 a $(1933)$.

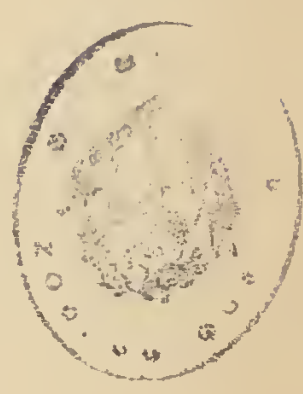


purpuraria J. Joann., Lép. Hét. Mascar. (Livre Mauritius Cent. Soc. Ent. Fr.), p. 438 (1932).

distracta Walk., List Lep. Ins., 22, p. 594 (1861) Zentral(Thalera) (Brazil).

? ol ivaria Snell., Tijd. Ent., 17, p. 46, t. Amerika bis Peru, Brasilien 3 , f. 8 (1874) (Zonosoma) (sine loc. [?Columbien]. - Druoe, Biol. Centr.-Amer., Lep. Het., 2, p. 102 (1892) (Ephyra). - Kaye \& Lamont, Trin. Lep. Het., p. 109 (1927) (Mn.).

the tis part. Warr., Nov. Zool., 7, p. 158 (1900).

cryptereuthus Prout, Ann. Mag. Nat. Hist., $8: 8$, p. 705 (1911) (N.).

(Colombia)

var. (?) thet is Warr., Nov. Zool., 7, p. 158 Costa-Rica (1900) (Costa Rica).

bis

Ecuador

deaurata Warr., Nov. Zool., 11, p. 34 (1904) (Craspedia) (N. W. Ecuador).

simplicearia Druce, Biol. Centr.-Amer.,

Zentral-

Amerika

bis

Ecuador Lep. Het., 2, p. 94 (1892) (Amaurinia) (err. det., nec Walk.).

rufipuncta Warr., Proc. U. S. Nat. Mus., 30, p. Süidost452 (1906) (Mn.). - Prout, Nov. Zool., 25, Brasilien p. $86(1918)$.

mys Prout, Nov. Zool., 25, p. 87 (1918).

Fr.-

Guayana

dentatilinca Warr., Nov. Zool., 11, p. 515 (1904) Jamaika, (Trichosterrha?) (Venezuela).

Panama

bis

Ecuador,

Venezuela

idaea Swinh., Tr. Ent. Soc. Lond., 1892, p. 6 (1892) (Hemithea) (Khasia Hills); 1894, id., l. c., 1894, p. 179 (1894) (Dithalma); id., Cat. Lep. Het., Oxf. Mus., 2, p. 370 (1900) $\left(M n_{0}\right)$. - Hampson, Faun. Ind., Moths, 3, p. 452 (1895) (Erythrolophus). - $\Lambda$. Pagenst., Jahrb. Nass. Ver. Nat., 51, p. 200 (1898) (Erythrolophus). - Rothsch., Journ. F. M. S. Mus., 8 : 3, p. 139 (1920).

inornata Warr., Nov. Zool., 3, p. 379 (1896) (Rho- Java, dostrophia) (Java). - Rothsch., Journ. F. Sumatra M. S. Mus., $8: 3$, p. 139 (1920).

phacnomeris Prout, Nov. Zool., 25, p. 77 (1918). Br.-Neu-

inconspicua Warr., Nov. Zool., 14, p. 142 Sikkim, Assan, Pahang, Sumatra, Lombok (1907) (Eu.) (err. det., nec. Warr. 1899). guinea 
inconspicua Warr., Nov. Zool., 6, p. 338 (1899) (Sym- Holl.- u. macra) (Milne Bay). - Prout, Nov. Zool., Br.-Neu25, p. 78 (1918); id., Ins. Samoa, 3:3, p. guinea 16 123 (1928).

\section{Genus Neonemoria Warr.}

Warr., Nov. Zool., 11, p. 23 (1904). - Prout, Gen. Ins., 129, p. 249 (1912).

thalassinata Snell., Tijd. Ent., 17, p. 54, t. 4, f. 3 Columbien (1874) (Acidatia) (Rio Magdalena).

virescens Th.-Mieg, Lo Nat., 14, p. 235 (1892) (Acidalia) (Colombie).

plana Warr., Nov. Zool., 11, p. 24 (1904) (Bolivia).

var. (?) ras a Warr., Nov. Zool., 7, p. 150 Vienezuela, (1900) (Venezuela).

ab. (?) decolor Warr., Proc. U. S. Mus., 30, p. 452 (1906) (Mnesithetis).

bis

Br.-

Guayana, Amazonas

(Demerara)

\section{Genus Symmacra Warr.}

Warr., Nov. Zool., 3, p. 116 (1896) (typus regularis Warr.). Prout, Ins. Samoa, 3 : 3, p. 123 (1928).

regularis Warr., Nov. Zool., 3, p. 116 (1896). Hampson, Journ. Bomb. Nat. Hist Soc.,
$12: 4$, p. 83 (1898) (Craspedia). - Prout, Ins. Samoa, $3: 3$, p. 123 (1928).

inobtrusa Warr., Nov. Zool., 4, p. 393 (1897) (Mnesithetis). - Prout, Ins. Samoa, $3: 3$, p. 123 (1928) (pr. var.?).

solidaria Guen., Spec. Gén. Lép., 9, p. 348 (1858) (Nemoria) (Ceylon). - Ch. Oberth., Et. Lép. Comp., 12, p. 79 , t. 383 , f. 3215 (1916) (Nemoria). - Prout, Ins. Samoa, 3:3, p. 123 (1928). - Sterneck, Iris, $42: 2$, p. 138 (1928).

quadra q a ta Walk., List Lep. Ins., 23, Khasiberge

Süd-

Celebes

Ceylon,

Assam u.

West-

China

bis

Queensland

und

Gundalcanar

p. 762 (1861) (Acidalia) (Borneo). - Swinh., Cat. Lep. Het. Oxf. Mus., 2, p. 360 (1900) (Craspedia).

va lidaria Walk., List Lep. Ins., 35, p. 1607 (1866) (Thalassodes) (Celebes). - Hampson, Faun. Ind., Moths, 3, p. 446 (1895); id., Journ. Bomb. Nat. Hist. Soc., $21: 4$, p. 1248 (1912) (Ephyra). - Swinh., Cat. Lep. Het. Oxf. Mus., 2, p. 370 (1900) (Mnesithetis). - Turn., Proc. Linn. Soc. N. Sth. 
Wales, 32, p. 671 (1908) (Sterrha). - Prout, Gen. Ins., 129, p. 73 (1912) (Sterrhu?).

tha la s sica Moore, Lep. Ceyl., 3, p. 430, t. 194, f. 6 (1887) (Jodis). - Swinh., Tr. Ent. Soc. Lond., 1894, p. 175 (1894) (Euchloris). o chre a Warr., Nov. Zool., 4, p. 393 (1897) (Mnesithetis) (Woodlark I.).

rufifrontaria Hampson, Journ. Bomb. Nat. Hist. Soc., $14: 4$, p. 655 (1907) (Geometra) (India).

plumbearia Sterneck, Iris, $41: 2$, p. 159 (1927) (Acidalia) (err. det., nec Leech) (Westchina) (var.?).

var. b a pt at a Wart., Nov. Zool., 4, p. 224 Fidji, (1897) (Sterrha?) (Apia). - Prout, Ins. Samoa Samoa $3: 3$, p. 123 (1928).

Craspedia sp., Reb., Denks. Akad. Wien, Math.-Nat. Kl., 85, p. 429 (1910). - Prout, 3 Ins. Samoa, $3: 3$, p. 123 (1928).

\section{Genus Pylargosceles Prout}

Prout, Nov. Zool., 35 : 4, p. 296, figg. (1930).

steganioides Butl., Пl. Het., 2, p. IX, 51, t. 37, China, f. 8 (1878) (Acidalia) (Japan). - Leech, Korea, Ann. Mag. Nat. Hist., (6) 20, p. 103 (1897) Japan (Acidalia). - Prout in Seitz, Macrolep., 4, p. 54, t. $4 \mathrm{~m} \mathrm{(1913)} \mathrm{(Acidalia);} \mathrm{id.,} \mathrm{Nov.}$ Zool., $35: 4$, p. 296 (1930). - Sterneck, Iris, $41: 2$, p. 156 (1927) (Acid. steganoides [err. typogr.]). - Marumo, Journ. Coll. Agric. Tokyo, 8 (2), p. 157 (1927) '(Scopula). ab. $u$ nic ol or Prout in Seitz, Macrolep., 4, p. 55 (1913) (Acidalia) (sine loc.).

Bi ol og i e: Matsumura, Oyo Konclingaku (ed. $2)$, t. 29 , f. 5 (1920).

limbaria Wileman, Entom., 48, p. 81 (1915) (Pty- Formosa chopoda). - Prout, Nov. Zool., $35: 4$, p. 2 296 (1930) (pr. subsp.?).

\section{Genus Tricentra Warr.}

Warr., Nov. Zool., 7, p. 164 (1900) (typus flavimarginata Warr.). Pa m meris Warr., Proc. U. S. Mus., 30, p. 454 (1906) (typus albiguttata Warr.).

fumata Warr., Proc. U. S. Mus., 30, p. 454 (1906) Fr.(Pammeris).

Guayana

albiguttata Warr., Proc. U. S. Mus., 30, p. 454 Fr.(1906) (Pammeris) (French Guiana). Guayana bis Peru 
albigutta Dyar, Proc. U. S. Mus., 47, p. 235 (1914) (Pammeris).

euriopis Dyar, Proc. U. S. Mus., 47, p. 235 (1914) Panama, (Pammeris).

Guayana

? Fr.-

mimula Warr., Nov. Zool., 14, p. 223 (1907). Süd-Ost-

Peru

aurilimbata Warr., Proc. U. S. Mus., 30, p. 462 Fr.-

(1906).

vinosata Warr., Proc. U. S. Mus., 30, p. 466 (1906). Fr.-

Guayana

flavimarginata Warr., Nov. Zool., 7, p. 165 (1900). Ecuador

subplumbea Bastelb., Ent. Zeits. Stuttg., 22, p. 58 Bolivien (1908).

angulisigna Dogn., Ann. Soc. Ent. Belg., 52, p. 265 Guayana, (1908) (Guyane franȩ.).

Amazonas

bisignata Warr., Nov. Zool., 14, p. 222 (1907). Süd-Ost-

quadrigata $\mathrm{C}$. Felder, Reise Novara, Irep. Het., t. Panama, 128 , f. 9 (1875) (Acidalia) (Amazon). - Venezuela, Dogn., Ann. Soc. Ent. Belg., 52, p. 266 Guiana, (1906).

Amazonas,

? Nord-

albipunctata Druoe, Biol. Centr.-Amer.,

Argentinien

Iep. Het., 2, p. 539, t. 99, f. 14 (1899) (Eois) (Panama).

unimaeula Dogn., Hét. Nouv. Amér. Sud, 4, p. 8 Fr.(1911).

Guayana

ignefumosa Warr., Proc. U. S. Mus., 30, p. 465 Holl.(1906) (Tranz.-Guiana). u. Fr.-

Guayana

debilis Dogn., Ann: Soc. Ent. Belg., 50, p. 111 Holl.(1906) (Guyane franç.).

Guayana

fulvifera Dogn., Ann. Soc. Ent. Belg., 52, p. 265 Fr.(1908).

eonsequens Warr., Proc. U. S. Mus., 30, p. 464 Fr.(1906) (sequ. var.?).

aseantia Druce, Biol. Centr.-Amer., Lep. Het., 2, Guatemala p. 109, t. 51, f. 20 (1892) (Eois).

ellima Schaus, Tr. Amer. Ent. Soc., 27, p. 259 (1901) Mexiko (Eois).

anctidisea Prout, Nov. Zool., 25, p. 82 (1918). Fr. 
llavimargo Warr., Nov. Zool., 12, p. 326 (1905) (Co- Panama, lombia).

carnaria Druce, Biol. Centr.-Amer., Lep. Het., 2, p. 108 (1892) (Eois) (err. det., neo H.-Sch.).

gibbimargo Prout, Nov. Zool., 25, p. 83 (1918).

Fr.-

Guayana

commixta Wart., Nov. Zool., 12, p. 325 (1905). Peru

cambogiata Warr., Nov. Zool., 4, p. 437 (1897) Trinidad, (Eois?) (Brit.-Guiana). Venezuela, Guayana, Amazon

citrinaria Warr., Nov. Zool., 14, p. 222 (1907) (S. T. Ecuador, Peru).

Peru

var. grisescens Prout, Nov. Zool., 24, p. Amazonas, 388 (1917) (Fonteboa). Fr.-

Guayana

carnaria H.-Sch., Samml. Außereur. Schmett., $1: 5 /$ 10, t. [39], f. 194 (1854) (Acidalia) (SuIfinam); $18 / 22$, p. 36 (1856) (Arrhostia); Schluß-Feft, p. 61 (Arrhostia), p. 80 (1858) (Acidalia). - Walk., List Lep. Ins., 22, p. 662 (1861) (Hyria?).

carnearia Guen., Spec. Gén. Lép., 9, p. 431 (1858) (Hyria?).

laciniata Warr., Nov. Zool., 14, p. 223 (1907) (S. E. Peru).

allotmeta Prout, Nov. Zool., 24, p. 387 (1917). Süd-Ost-

Surinam,

Süd-Ost-

Peru

Peru

flavicurvata Dogn., Ann. Soc. Ent. Belg., 54, p. Bolivien $115(1910)$.

flavifigurata Prout, Nov. Zool., 24, p. 388 (1917). Süd-Ost-

Peru

devigescens Prout, Nov. Zool., 24, p. 387 (1917) Surinam, (Surinam). Peru

benevisio Prout, Nov. Zool., 37, p. 230 (1932). Ost-

Bolivien

gavisata Walk., List Lep. Ins., 26, p. 1587 (1862) Costa-Rica (IIyria) (Amazon).

bis

Guayana,

Amazonas,

ne c u la Druce, Biol. Centr.-Amer., Lep. Het. u. Peru 2 , p. 114 , t. 52 , f. 9 (1892) (Cambogia?) (Panama).

brunneomarginata Warr., Proo. U. S. Mus., 30, p. Surinam, 463 (1906) (French Guiana).

Fr.-

Guayana,

Nord-Ost-

Brasilien 
decorata Warr., Nov. Zool., 12, p. 326 (1905). Ecuador

oeno Druce, Biol. Centr-Amer., Lep. Het., 2, p. Mexiko 110 , t. 51, f. 28 (1892) (Eois?).

biguttata Warr., Proc. U. S. Nat. Mus., 30, p. 462 Fr.(1906).

Guayana

apicala Dogn., Ann. Soc. Ent. Belg., 54, p. 115 Bolivien (1910).

protuberans Dogn., Ann. Soc. Ent. Belg., 54, p. Bolivien 114 (1910).

flavitornata Prout, Bull. Hill Mus., $1: 2$, p. 260 Fr.(1922); $1: 3$, p. 624 , t. 25 , f. 9 (1924). Guayana

ocrisia Druce, Biol. Centr.-Amer., Lep. Het., 2, p. Guatemala 122 , t. 52 , f. 27 (1892) (Acidalia?).

amibomena Prout, Nov. Zrool., 25, p. 83 (1918) Fr.(Tricentra?) (French Guiana). Guayana,

Amazon,

Ost-

Bolivien

percrocea Warr., Proc. U. S. Nat. Mus., 30, p. 465 Fr.41 (1906) (French Guiana) (huj. gen.?).

Guayana, Peru, ? OstBrasilien

\section{Genus Anthemoctena Warr.}

Warr., Nov. Zool., 2, p. 102 (1895). - Janse, Check-List S. Afr. Lep. Het., p. 105 (1917). - Prout in Seitz, Macrolep., 16, p. 49 (1933).

textilis Wllgr., Ofv. K. Vet. Ak. Förh., 29:3, p. Transvaal 60 (1872) (Mesotype) (Caffraria or.). - bis Janse, Check-List S. Afr. Lep. Het., p. 105 Kapland (1917). - Prout in Seitz, Macrolep., 16, p. 49 , t. 6 a (1933).

line a ta Warr., Nov. Zool., 2, p. 102 (1895) 1

(S. Africa). - Janse, 1. c. (1917).

\section{Genus Eryfhrolophus Swinh.}

Swinh., Tr. Ent. Soc. Lond., 1892, p. 10 (1892).

Erythrolophus sect. O. (part.) Hampson, Faun. Ind., Moths, 3, p. 454 (1895).

Iasclcorpus Swinh., Tr. Ent. Soc. Lond., 1892, p. Assam, 11 (1892) (Khasia); 1894, p. 180 (1894) Tonkin, (Erithrolophus). - Hampson, Faun. Ind., Malay.Moths, 3, p. 454 (1895). - J. Joann., Ann. Halbinsel, Soc. Ent. Fr., $98: 4$, p. 477 (1929). Java $f$ u s cicor $p$ us Candèze, Encycl. Lép., $2: 3 / 4$, 


\section{Genus Semaeopus H.-Sch.}

H.-Sch., Samml. Außereur. Schmett., 1 : (13/17, f. 327, indescr.); $18 / 22$, p. 25, 36 (1856) (typus serrilinearia H.-Sch.). - Warr., Nov. Zool., 2, p. 91 (1895). - Prout, Nov. Zool., 24, p. 378 (1917).

Cyphoptery $x$ Guen., Spec. Gén. Lép., 9, p. 404 (1858) (typus incurvaria Guen.).

$C n$ e $m$ odes Guen., op. cit. 10, p. 5 (1858) (typus indignaria Guen.). - Walk., List Lep. Ins., 23, p. 802 (1861). - Warr., Nov. Zool., 2, p. 91 (1895). - Prout, Ann. Mag. Nat. Hist., (8) 6, p. 242 (1910) (Semaeopus sect. Cnemodes).

I s s a Walk, Journ. Linn. Soc. Zool., 9, p. 198 (1867) (typus munda Walk.).

L ig o n i a Möschl., Verh. Zool.-Bot. Ges. Wien, 31, p. 399 (1881) (typus exquisitata Möschl.).

Dichromatopodia Warr., Nov. Zool., 2, p. 94 (1895) (typus sigillata Wulk.).

Dysephyra Warr., 1. c. (1895) (typus discopunctaria H.Sch.).

Heterephyra Warr., t. c., p. 95 (1895) (typus lateritiaria Warr., nec H.-Sch.).

Ch a r o m m a ta e a Hulst, Tr. Amer. Ent. Soc., 23, p. 302 (1896) (typus ella Hulst.).

Xenostigma Warr., Nov. Zool., p. 165 (1900) (typus perfusaria Walk.).

Schistocolpia Warr., Proc. U. S. Mus., 35, p. 460 (1906) (typus crinita Warr.).

Paradmeta Warr., Nov. Zool., 14, p. 221 (1907) (typus perstrigata Warr.).

Parazeux is Warr., Nov. Zool., 14, p. 221 (1907) (typus punctigera Dogn.).

$D a s y c o s y m b i a$ Großbeck, Journ. N. Y. Ent. Soc., 20, p. 283 (1912) (typus gracilata Großbeck).

Inficeta Dogn., Ann. Soc. Ent. Belg., 41, p. 216 Ecuador (1900) (Acidalia). - Wari., Proc. U. S. Mus., 30, p. 461 (1906) (Sterrha).

punctigera Dogn., Ann. Soc. Ent. Belg., 44, p. 440 Columbien (1900) (Anisodes) (Ecuador). - Warr., Nov. bis Zool., 14, p. 221 (1907) (Parazeuxis). - Ost-Peru Prout, Nov. Zool., 24, p. 378 (1917) (puntigera, err. typogr.).

itcolorata Warr., Nov. Zool., 11, p. 514 (1904). Bolivien

noverca Dogn., Ann. Soc. Ent. Belg., 44, p. 441 Ecuador (1900) (Dysephyra).

Irygodata Warr., Nov, Zool., 11, p. 36 (1904) Peru, (Craspedia) (Bolivia). - Prout, Nov. Zool, Bolivien 24, p. 378 (1917).

verbena Dogn., Ann. Soc. Ent. Belg., 37, p. 158 Columbien, (1893) (Anisodes) (Ecuador). Ecuador

subtranslucens Prout, Ann. Mag. Nat. Hist., (9) 2, p. 413 (1918).

(Colombia)

serrilinearia H.-Sch., Samml. Außereur. Schmett., Brasilien $1: 13 / 17$, t. [59], f. 327 (1855); 18/22, p. 36 (1856). - Warr., Nov. Zool., 2, p. 91 (1895). 
h i r ca ria Guen., Spec. Gén. Lép., 10, p. 5 (1858) (Cn.). - Walk., List Lep. Ins., 23, p. 803 (1861) (Cn.).

castaria Guen., Spec. Gén. Lép., 10, p. 5 (1858) Cuba, (Cn.) (Haiti). - Walk., List Lep. Ins., Jamaika, 23, p. 802 (1861) (Cn.). - Warr., Nov. Haiti Zool., 2, p. 91 (1895). - Cl. Oberth., Et. Lép. Comp., 20, p. 216, t. 549, f. 4642 (1923) (Cn.).

so litaria Walk., List Lep. Ins., 22, p. 728 (1861) (Acidalia) (S. Domingo).

con c in nata C. Felder, Reise Novara, Lep. Het., 2, t. 128, f. 15 (1875) (Acidalia) (Haiti).

malefidaria Möschl., Abl. Senck. Nat. Ges., 16, p. Porto-Rico 241 (1890) (Cn.) (= praec.?).

dentilinea Warr., Nov. Zool., 7, p. 149 (1900) (Cn.) Costa-Rica (W. Ecuador).

ab. carnearia Warr., Nov. Zool., 11, p.

Bolivien,

Süd-Ost-

Brasilien

513 (1904) (S. E. Peru). - simplex Warr., Nov. Zool., 12, p. 320 (1905) (Cn.) (S. E. Peru).

ladrilla Dogn., Ann. Soc. Ent. Belg., 37, p. 161 (1893) (Ephyra); id., Lép. Loja, 3, p. 75 (1894) (Anisodes) (Ecuador).

directilinea Schaus, Ann. Mag. Nat. Hist., (8) 10, p. 297 (1912) (H.) (Costa Rica).

indignaria Guen., Spec. Gén. Lép., 10, p. 6 (1858) Cuba, (Cn.) (Brésil? [err. loc.]). - Walk., List Haiti Lep. Ins., 23, p. 803 (1861) (Cn.). - Ch. Oberth., Ft. Lép. Comp., 20, p. 216, t. 549, f. 4645 (1923) (Cn.). - Prout, Nov. Zool., $36: 2$, p. 175 (1931).

a b s conditaria Walk., List Lep. Ins., 26, p. 1488 (1862) (Drepanodes) (S. Domingo).

var. (?) filifer $a t a$ Walk., List Lep. Ins., Jamaika 26, p. 1573 (1862) (Ephyra).

mieropis Hampson, Ann. Mag. Nat. Hist., (7) 14, Bahamap. 179 (1904).

Inseln

fuseifrons Warr., Proc. U. S. Nat. Mus., 30, p. Cuba 436 (1906) (Cremodes) (err. typogr.) (sequ. syn.?).

perletaria Möschl., Abh. Senck. Nat. Ges., 16, p. Porto-Rico 240 (1890) (Cn.) (Porto-Rico).

exypna Prout, Nov. Zool., 24, p. 382 (1917) (sequ. Ost-Peru var. vel syn.?).

alleia Schaus, Tr. Amer. Ent. Soc., 27, p. 191 Süd-Ost(1901) (Ephyra).

Brasilien

perspeetaria Walk., List Lep. Ins., 22, p. 634 (1861). Panama, (Ephyra?) (Venezuela).

ab. flavida Warr., Nov. Zool., 14, p. 217 (1907) (Dys.) (Venezuela)

eoncomitans Warr., Proc. U. S. Mus., 30, p. 435 Cuba (1906) (Cremodes) (err. typogr.). 
curta Warr., 1. c. (1906) (Cremodes) (err. ty- Cuba pogr.).

plerta Schaus, Tr. Amer. Ent. Soc., 27, p. 192 (1901)i Mexiko (Cn.).

sabuloides Schaus, 1. c. (1901) (Cn.). Mexiko

pertumna Schaus, t. c., p. 191 ( $C_{n}$.) (Peru). Venezuela,

Columbien

bis

Bolivien

viridiplaga Walk., List Lep. Ins., 23, p. 799 (1861) Brasilien, (Timandra) (Brazil). - Schaus, Proc. Zool. Paraguay, Soc. Lond., 1896, p. 645 (1896) (Timandra). Argentinien - Warr., Nov. Zool., 14, p. 216 (1907) (Cn.). - Prout, Ann. Mag. Nat. Hist., (8) 6, p. $242(1910)$.

ab. vacuat a Warr., 1. c. (1907) (Cn.) (Paraguay).

ab. a nfractata. Prout, t. c., p. 241 (1910). (Paraguay)

enodiflexa Prout, Nov. Zool, $36: 2$, p. 175 (1931) Paraguay, (Paraguay).

Süd-Ost-

Brasilien

? Nord-

indignaria Prout, Ann. Mag. Nat. Hist., Argentinien

(8) 6, p. 241 (1910); id., 1. c., (9) 1, p. 20

(1918) (err. det., nec Guen.).

ciliata Prout, Ann. Mag. Nat. Hist., (9) 1, p. 20 Panama

(1918) (Panama); id., Nov. Zool., $36: 7$, p. 175 (1931).

\section{Bolivien,} Paraguay,

Brasilien

? indignaria Warr., Nov. Zool., 2, p. 91 (1895) (Cn.). - ? Dyar, Proc. U. S. Nat. Mus., 47, p. 231 (1914) (Cn.) (err. det., nec Guen.).

rubripuncta Dogn., Ann. Soc. Ent. Belg., 46, p. Nond344 (1902) (Cn.) (Tucuman). - Prout, Tr. Argentinien, Ent. Soc. Lond., 1910, p. 214 (1910); id., Minas Mitt. Münchn. Ent. Ges., 21, p. 18 (1931). Geraes

semieaeca Prout, Nov. Zool., 24, p. 378 (1917). AmazonenStrom

anomala Dogn., Ann. Soc. Ent. Belg., 46, p. 343 Süd(1902) (S. ?).

Columbien

errabunda Dogn., Ann. Soc. Ent. Belg., 44, p. 441 Ecuador $(1900)$ (Dys.); id., l. c., 46, p. 344 (1902) (S.).

munda Walk., Journ. Linn. Soc. Zool., 9, p. 198 Columbien (1867) (Issa) (Bogota).

f. (?) signifer Warr., Nov. Zool., 11, p. Venezuela, 42 (1904) (Bolivien).

Columbien

bis

Bolivien 
vinodiscata Dogn., Mém. Sac. Ent. Belg., 22, p. 10 Ost(1913) (Cn.) (pr. f.?).

Columbien

Smithi Prout, Ann. Mag. Nat. Hist., (9) 1, p. 20 Columbien (1918).

illimitata Warr., Nov. Zool., 7, p. 156 (1900) (Hete- Zentralrephyra) (W. Ecuador). - Dyar, Proc. U. Amerika S. Nat. Mus., 47, p. 231 (1914) (Metere- bis Peru, phyra). - Kaye \& Lamont, Cat. Trin. Lep. Trinidad, Het., p. 108 (1927).

lateritiaria? Butl., Tr. Ent. Sac. Lond., Guayana, Ámazonas, Matto-Grosso 1881, p. 333 (1881) (Anisodes) (nec H.-Sch.). globaria ? Butl., l. c., p. 334 (1881) (Anisodes) (nec Guen.).

ple norbis (Warr. M. S.) Dogn., Ann. Soc. Ent. Belg., 37, p. 161 (1893) (Ephyra) (indescr.).

a tz idiscata Warr., Proc. U. S. Mus., 30, p. 442 (1906) (Dys.) (Cayenne).

ab. nigridiscata Warr., ibid. (1906) (Dys.) (Cayenne).

var. fl a vicans Prout, Nov. Zool., 24, p. 379 Nord(ladrilla subsp.) (1917).

Venezuela

discosa Dyar, Ins. Ins. Mens., 6, p. 136 (1918) Mexiko (? sequ. ab.; ? concatenans ab.).

cltrina Druce, Biol. Centr-Amer, Iep. Het., 2, Mexiko p. 102, t. 51, f. 2 (1892) (Ephyra).

concatenans Dyar, Ins. Ins. Mens., 6, p. 136 (1918). Mexiko

testacea Dogn., Mém. Soc. Ent. Belg., 22, p. 10 Columbien (1913) (Het.).

lutea Dogn., Ann. Soc. Ent. Belg., 53, p. 88 (1909) Ecuador (Dys.).

cuthyoria Prout, Nov. Zool., 24, p. 379 (1917). Amazonas

pustulata Warr., Nov. Zool., 11, p. 377 (1904) (Ilet.). Süd-Ost- Prout, Nov. Zool., 25, p. 85 (1918). Peru, ? Columbien

var. a urantirufa Prout, 1. c. (1918) Brit.(Costa-Rica).

Honduras, Costa-Rica

perpolitaria Möschl., Verh. Zool.-Bot. Ges. Wien, Venezuela 31, p. 406, t. 17, f. 16 (1881) (Anisodes) bis Fr.(Surinam). - Dogn., Ann. Soc. Ent. Belg., Guayana, 57, p. 63 (1913) (Dys.).

Matto-

Grosso,

Peru,

Bolivien

irmat a Ch. Oberth., Et. Lép. Comp., 6, p. 228,346 , t. 44, f. 1348 (1912) (Drepanodes?) (Matto-Grosso).

gracilata Grossbeck, Journ. N. Y. Ent. Soc., $20: 4$, Arizona p. 283 (1912) (Dasy.). - Barnes \& Mc Dunnough, Check List Lep. Bor. Amer., p. 101 (1917) (Dasy.). 
caecaria Hb., Verz. Bek. Schmett., p. 302 (1825) Mexiko (Codonia) [Surinam]. - Prout, Nov. Zool., bis 24, p. 383 (1917).

Peru, Venezuela bis Fr.-

Guayana,

Brasilien,

Nord-

pun c ta ta Stoll, Aanh. Oramers Uitl. Kap., 2, Argentinien p. 58, t. 12 , f. 10 (1790) (nom. praeocc., nec Clerck, 1759), - Verloren, Cat. Ins. Iep. Cram., p. 156 (1837) (Phalaena). Druce, Biol. Centr-Amer., Lep. Het., 2, p. 101 (1892) (Ephyra punctaria). - Kaye \& Lamont, Cat. Trin. Lep. Het., p. 108 (1927).

fartaria Guen., Spec. Gén. Lép., 9, p. 406 (1858) (Ephyra). - Oh. Oberth., Et. Lép. Comp., 12, p. 150, t. 394, f. 3342 (1916) (Ephyra).

ab. grisea Warr., Proc. U. S. Nat. Mus., 30, p. 448 (1906) (Het.) (Trinidad).

var. (?) occipitaria H.-Sch., O.-B. Zool.- Cuba,

Min. Ver. Regensb., 24, p. 180 (1870) (Zonosoma) (Cuba). - Gdl., Ent. Cub., 1, p. 381 (1881) (Zonosoma). - occipitraria Möschl., Abh. Senck. Nat. Ges, 16, p. 235 (1890) (Zonosoma).

val. distinctata Warr., Nov. Zool., 7, p. Dominica, 155 (1900) (Het.) (Dominica). - rubella Sta. Lucia, Warr., Nov. Zool., 14, p. 220 (1907) (Het.) St. Vincent, (Dominica) (ab.).

Grenada

nossis Prout, Nov. Zool., 25, p. 84 (1918).

Venezuela,

subrubra Kaye, Tr. Ent. Soc. Lond., 1901, p. 148, t. 6, f. 19 (1901) (Het.) (Trinidad). Prout, Nov. Zool., 25, p. 84 (1918). - Kaye \& Lamont, Cat. Trin. Lep. Het., p. 108 (1927).

Panama,

Trinidad,

Guayana,

Amazon,

Paraguay

var. dominicana Prout, Nov. Zool., 25, p. Dominica 84 (1918) (sp. div.?). - pallida ab. purpurata (Warr. M. S.) Prout, 1. c. (1918).

argentipuncta Warr., Nov. Zool., 4, p. 435 (1897) Venezuela (Dichr.).

fuscicosta Warr., Nov. Zool., 7, p. 155 (1900) (Het.) Costa-Rica, (Ecuador). $\quad$ Fcuador

johannis Schaus, Ann. Mag. Nat. Hist., (8) 10, p. 297 (1912) (Het.) (Costa Rica).

compularia Snell., Tiju. Ent., 17, p. 55, t. 4, f. Columbien 4 (1874) (Acidalia).

dorsiornata Prout, Nov. Zool., 24, p. 380 (1917). Goyaz

mesoturbata Dyar, Proc. U. S. Mus., 44, p. 313 Mexiko (1913) (Anisodes).

palliata Warr., Proc. U. S. Mus., 34, p. 108 (1908) Fr.(Thysanopyga). 
orbifcra Prout, Nov. Zool., 27, p. 269 (1920). Fr.-

Guayana

decorata Dogn., Ann. Soc. Ent. Belg., 53, p. 88 Fr.(1909) (Het.) (Guyane franç.).

Peru,

Bolivien,

Matto-

Grosso

semibrunnea Warr., Proc. U. S. Mus,, 30, p. 449 Fr.(1906) (Ilet.) (French Guiana). - Dyar, Guayana, ibid., 47, p. 231 (1914) (Heterephyra). Panama

fulvescens Warr., Proc. U. S. Mus., 30, p. 448 Costa-Rica (1906) (Heterephyra) (French Guiana). bis

Amazon, ? Paraguay (var.?)

lunifera (part.) Warr., Nov. Zool., 4, p. 436 (1897) (Dysephyra).

e rastus Schaus, Ann. Mag. Nat. Hist., (8) 10, p. 292 (1912) (Anisodes).

fusilinca Dogn., Ann. Soc. Ent. Belg., 53, p. 88 Fr.(Costa Rica) (1909) (Het.).

Guayana

aurata Warr., Proc. U. S. Mus., 30, p. 447 (1906) Fr.(Het.A.

bobaria Dogn., Le Nat., 12, p. 58 (1890); id., Lép. Ecuador Loja, 2, p. 61, t. 6, f. 12 (1891) (Melinodes).

lunifera Warr., Nov. Zool., 4, p. 436 (1897) (Dys- Columbien ephyra) (Columbien); id., Proc. I. S. Nat. bis Mus., 30, p. 448 (1906) (Het.) (praec, Bolivien, form.?).

Amazonas, Brasilien

luridata Warr., Nov. Zool., 14, p. 219 (1907) (Het.). Süd-Ost- Prout, Nov. Zool., 24, p. 380 (1917). Peru

subrugosa Prout, Nov. Zool., 24, p. 380 (1917) (lu- Amazonas, ridata var:) (Amazons).

Bolivien,

Matto-

Grosso

commaculata Warr., Nov. Zool., 4, p. 435 (1897) Brit.-

(Dys.) (Brit.-Guiana); 8, p. 457 (1901) Guayana

(Het.). - Prout, Nov. Zool., 24, p. 381 bis

(1917).

Bolivion,

Brasilien

hypoderis Prout, Nov. Zool., 24, p. 380 (19177) Süd-Ost-

(S. E. Peru).

Trinidad

(var.),

Süd-Ost-

Brasilien

(var.?) 
duplicata Warr., Nov. Zool., 12, p. 323 (1905) Columbien (Het.).

peplumaria Schaus, Ann. Mag. Nat. Hist., (8) 10, Honduras, p. 293 (1912) (Costa Rica). Costa-Rica

catamompha Prout, Nov. Zool., 24, p. 381 (1917). Ost-

Bolivien

neximargo Warr., Nov. Zool., 8, p. 457 (1901) (Het- Ost-Peru, erephyra) (Ostperu).

Ost-

Bolivien

mira Prout, Nov. Zool., $36: 2$, p. 176 (1931). Ost-Peru

exquisitata Mösch1., Verh. Zool.-Bot. Gres. Wien, 31, Panama, p. 399 (1881); id., l. c., 33, p. 358, t. 18, f. Guayana, 41 (1883) (Ligonia) (Surinam). - Dyar, UnterProc. U. S. Nat. Mus., 47, p. 232 (19̃14) Amazon ( Lig.).

plumbeostrola Prout, Nov. Zool., 24, p. 382 (1917) Triuidad, (Surinam).

Venezuela,

Guayana,

Amazonas,

Ost-Peru,

Bolivien

geminilinca Prout, Nov. Zool., 24, p. 382 (1917) Amazonas, (Amazons).

mitranaria Walk., List Lep. Ins., 20, p. 241 (1860) Fr.-

(Epione?) (Brazil). - Warr., Nov. Zool., Guayana, 2, p. 97 (1895) (Lipomelia). Amazon,

Süd-Ost-

Peru

simplicilinea Prout, Nov. Zool., 27, p. 269 (1920) Ost-Peru, (Bolivien).

Bolivien

seriptilinea Schaus, Ann. Mag. Nat. Hist., (8) 10, Costa-Rica, p. 293 (1912) (Costa-Rica). Tenezuela

purpurcoplaga Prout, Nov. Zool., 24, p. 385 (1917) Brasil.(S.?). $\quad$ Amazon

pallida Warr., Nov. Zool., 2, p. 94 (1895) (Dich.). Amazon

discors Prout, Nov. Zool., 25, p. 85 (1918) (Co- Columbien, lumbien).

Brit.-

Guayana,

Amazonas,

Bolivien

incurvaria Guen., Spec. Gén. Lép., 9, p. 404 (1858) Brasilien

(Cyph.). - Ch. Oberth., Et. Lép. Comp.,

12, p. 150 , t. 394 , f. 3339 (1916). - Prout,

Nov. Zool., 25, p. 86 (1918). (Cyph.).

olivaceonotăta Warr., Nor. Zool., 4, p. 435 (1897) Venezuela, (Dichr.) (Venezuela). 
paulena Schaus, Tr. Amer. Ent. Soc., 27, p. 189 Süd-Ost(I90I) (Dichr.) (S.-O. Brasilien). Brasilien,

Paraguay

deflexa Warr., Nov. Zool., 7, p. 152 (1900) (Dichr.) Columbien, (Venezuela). - Prout, Nov. Zool., 25, p. 86 Venezuela (i918).

canidiscata Warr., Nov. Zool., 14, p. 217 (1907) Columbien, (Dichr.) (Paraguay) (praec. form.?). Guayana,

Ecuador,

Bolivien,

Argentinien

bis

Süd-Ost-

deflexa ab. griseomaculata Dogn., Ann. Soc. Ent. Belg., 54, p. 114 (1910) (Dichr.).

sigillata ab. flexilinea Warr., M. S.

naltona Schaus, Tr. Amer. Ent. Soc., 27, p. 189 Mexico (1901) (Dichr.) (Venezuela). - Dyar, Proc. bis U. S. Nat. Mus., 47, p. 232 (1914) (Het.) Panama, (praec. form.?).

Venezuela

offlexa Prout, Nov. Zool., 25, p. 86 (1918).

Amazonas

concomitata nom. nov.

concomitans Warr., Nov. Zool,, 14, p. 217

(1907) (Dichr.). - Prout, Tr. Ent. Soc. Lond., 1910, p. 216 (1910) (Dichr.) (nom. praeocc.):

Nord-

Argentinien,

? Bolivien,

? Matto-

Grosso

oaxacana Schaus, Tr. Amer. Ent. Soc., 27, p. 189 (1901) (Dichr.) (Mexico). - Prout, Nov. Zool., 25, p. 86 (1918) (sequ. ab.?).

Mexico,

Columbien,

Venezuela,

distans Warr., Nov. Zool., 11, p. 36 (1904) (Dichr.) (Santa Marta).

osteria Druce, Biol. Centr.-Amer., Lep. Het., 2, p. Mexiko 537 , t. 99 , f. 9 (1899) (Anisodes? $)$. Prout, Nov. Zool., 25, p. 86 (1918).

masinissa Schaus, Ann. Mag. Nat. Hist., (8) 10, Costa-Rica, p. 298 (1912) (Dichr.) (Costa-Rica). Peru

Iodillaria Möschl., Verh. Zool.-Bot. Ges. Wien, 31, Venezuela p. 399 (1881) (Scardamia) (Surinam). bis Fr.-

rufimedia Warr., Proc. U. S. Mus., 30, p. 441 (1906) (Dichr.).

Guayana

(Cayenne)

ascia Prout, Nov. Zool., 25, p. 86 (1918) (Venezuela). Venezuela, Surinam,

Nord-

Argentinien,

Paraguay

decalvaria Möschl., Abh. Senck. Ges., $14: 3$, p. 70 Jamaika (1886) (Anisodes). 
r u be lla Warr., Nov. Zool., 2, p. 94 (1895) (Dichr.).

zova Schaus, Tr. Amer. Ent. Soc., 27, p. 189 (1901) Mexiko (Dichr.).

sigillata Walk., List Lep. Ins., 26, p. 1491 (1862) Amazonen(Pyrinia). - Warr., Nov. Zool., 2, p. 94 strom (1895) (Dichr.). - ?? Prout, Tr. Ent. Soc. Lond., 1910, p. 216 (1910) (Dichr.).

preplocyela Prout, Ann. Mag. Nat. Hist., (9) 1, p. Venezuela, 21 (1918) (Peru).

Amazonas, Peru

micipsa Schaus, Ann. Mag. Nat. Hist., (8) 10, p. Costa-Rica 298 (1912) (Dichromatopodia).

Hoffmannsi Prout, Nov. Zrool., 25, p. 86 (1918). Amazonas

ccrvina Warr., Proc. U. S. Mus:, 30, p. 441 (1906) Taboga(Dichr.) (Venezuela). - ? Dyar, ibid., 47, Insel, p. 231 (1914) (Dichromatopodia). Venezuela,

PEcuador

purpurea Warr., l. c. (1906) (Dichr.) (Fr. Guayana). Fr.-

Guayana, Amazonenstrom

miniata Druce, Biol. Centr.-Amer., Lep. Hot., 2, p. Panama, 538, t. 99, f. 12 (1899) (Anisodes?) (Pa- ?Guayana nama). - Schaus, Ann. Mag. Nat. Hist, bis (8) 10, p. 298 (1912) (Dichr.).

m un at a (err. typogr.) Warr., Proc. U. S. Nat. Mus., 30, p. 441 (1906) (Dichr.).

calavera Dogn., Ann. Soc. Ent. Belg., 41, p. 31 Columbien (1897) (Anisodes) (Ecuador).

terdilinea Prout, Nov. Zool., 24, p. 384 (1917). Venezuela, Bolivien (var.?)

hepaticata Warr., Nov. Zool., 12, p. 321 (1905) Columbien, (Dichr.) (Columbien).

Brit.-

Guayana,

Amazon

orbona Schaus, Ann. Mag. Nat. Hist,, (8) 10, p. Costa-Rica 298 (1912) (Dichr.).

fuscidiscaria Warr., Nov. Zool., 7, p. 156 (1900) S.-O.(Het.).

Brasilien,

F Fr:-

Guayana

(var.?)

subrosea Dogn., Ann. Soc. Ent. Belg., 44, p. 442 Ecuador (1900) (Dys.).

vestita Prout, Nov. Zool., 24, p. 383 (1917) (Vene- Columbien, zuela). 
var. asymphora Prout, t. c., p. 384 (1917) Peru, (Peru). - punctulifera Prout, 1. c. (1917) Bolivien, (Peru) (ab. vel. sp.?). Amazonas

maculimargo Dyar, Proc. U. S. Mus., 51, p. 30 Mexiko (1916) (Cn.).

ella Hulst, Tr. Amer. Ent. Soc., 23, p. 302 (1896) Arizona, (Char.) (Texas). - Barnes \& Mc Dun- Texas, nough, Check List Lep. Bor. Amer., p. Mexiko 101 (1917) (Dys.). - Prout, Nov. Zool., 24, p. 378 (1917).

pyraloides (part.), Druce, Biol. Centr.Amer., Lep. Het., 1, p. 412 (1890) (Hemeroplanis) (Chilpancingo) (err. det.!).

ab. ellatina Hulst, l. c., p. 303 (1896). (Char.).

dudisea Schaus, Tr. Amer. Ent. Soc, 27, p. 192 Mexiko (1901) (Cn.) (praec. form.?).

mizteca Schaus, l. c. (1901) (Cn.).

Mexiko

florera Dogn., Ann. Soc. Ent. Belg., 38, p. 132 Ecuador (1894) (Pellonia).

rubida Warr., Nov. Zool., 4, p. 439 (1897) (Het.). Venezuela - Prout, Nov. Zool., 24, p. 378 (1917).

var. orbistigma Prout, Nov. Zool., 24, p. Ecuador 383 (1917) (Peru). - roscigera Dogn., Iuép. bis Loja, 3, p. 75 (1894) (Anisodes) (err.det., Bolivien nec Walk.).

discopunctaria H.-Sch., Samml. Außereur. Schmett., Brasilien $1: 13 / 17$, t. [59], f. 331 (1855) (Dosithea); $18 / 22$, p. 36 (1856) (Arrhostia). - Guen., Spec. Génì Lép., 9, p. 417, t. 15, f. 5 (1858) (Anisodes). - ?? Dogn., Lép. Ioja, 3, p. 75 (1894) (Anisodes).

roseigera Walk., List Lep. Ins., 26, p. 1496 (1862) (Epione?).

trophinus Schaus, Ann. Mag. Nat. Hist., (8) 10, Costa-Rica, p. 296 (1912) (Anisodes) (Costa Rica). Peru

ephippiata Dogn., Ann. Soc. Ent. Belg., 57, p. 382 Panama (1914) (Het.) (praec. ab.?).

terlullus Schaus, t: c., p. 295 (1912) (Arisodes). Costa-Rica

rubellula Th.-Mieg, Le Nat., 14, p. 235 (1892) (Aci- Cayenne dalia).

justata Walk., List Lep. Ins., 22, p. 742 (1861) Brasilien (Acidalia). - Prout, Nov. Zool., 24, p. 378 (1917).

te p id a ta Walk., List Lep. Ins., 26, p. 1600 (1862) (Acidalia).

ochratipennis Warr., Nov. Zool., 12, p. 322 (1905) Venczucla, (Emmiltis) (Venezuela).

Mexiko,

Costa-Rica,

? St.

Vincent,

? Sta.

Lucia 
citrina Druce (part.), Biol. Centr.-Amer., Lep. Het., 2, p. 102 (1892) (Ephyra) (nec typ.).

ambagifera Warr., Nov. Zool., 4, p. 433 (1897) /Craspedia) (Venezuela); 12, p. 322 (1905) (Emmiltis). - Prout, Mitt. Münchn. Ent. Ges., 21 , p. 18 (1931).

Venezuela, Bolivien, Paraguay, Brasilien

var. (?) consobrinata Warr., Nov. Zool., Venezuela 7, p. 150 (1900) (Craspedia); 12, p. 322 (1905) (Emmiltis).

var. (?) e ublemmaria Ch. Oberth., Et. Bahia Lép. Comp., 20, p. 217, t. 549, f. 4643 (1923) (Somatina).

bimacula Warr., Nov. Zool., 4, p. 438 (1897) (Hae- Honduras, malea) (Brit.-Guiana). - Prout, Nov.Zool., Trinidad, 25 , p. 85 (1918).

Guayana,

Amazon,

Bolivien,

Süd-

Brasilien

redundala Prout, Nov. Zool., 25, p. 85 (1918). Süd-Ost-

Peru,

?Venozuela,

Prit.-

Guayana

Böltgeri Warr., Nov. Zool., 11, p. 513 (1904) (Li- Ost-Peru, gonia).

? Fr:-

Guayana

(var.?)

albipunctulata Dogn., Ann. Soc. Ent. Belg., 52, p. 17 (1908) (Heterephyra).

marginata Schaus, Tr. Amer. Ent. Soc., 27, p. 191 Mexiko (1901) (Char.).

nisa Druce, Biol. Centr.-Amer., Lep. Het., 2, p. Guatemala, 49 , t. 45, f. 24 (1892) (Acrotomia?) (Gu- Panama atemala). - Warr., Nov. Zool., 4, p. 439 bis (1897) (Lipomelia). - ? Kaye \& Lamont, Maranhao, Cat. Trin. Lrep. Het., p. 109 (1927) (Lipo- Mattomelia).

Grosso,

Peru,

Bolivien,

Paraguay

varia Warr., Nov. Zool., 2, p. 96 (1895) (Lipomelia?) (Br. Guiana).

\section{Guayana,}

Brasilien,

decorata Warr. M. S. (Autoglyphia Warr. M.S.).

sublineta Warr., Nov. Zool., 4, p. 439 (1897) (Li- Columbien, pomelia) (Bolivia).

Venezuela,

Amazonas,

Bolivien,

Paraguay

s u b f u s cata Warr., Nov. Zool., 11, p. 38 (1904) (Lipomelia).

(Bolivia) 
scripturata Warr., Proc. U. S. Mus., 30, p. 448 Fr.(1906) (Heterephyra) (Fr. Guiana). - Guayana, Prout, Nov. Zool., $37: 2$, p. 230 (1932). Amazonas

maleformata Prout, Nov. Zool., 37 :2, p. 230 (1932) Columbien, (Uruguay). Amazonas,

Uruguay

vizaria Schaus, Tr. Amer. Ent. Soc., 27, p. 194 Süd-Ost(1901) (Haemalea). Brasilion

perfusaria Walk., List Lep. Ins., 22, p. 634 (1861) Venezuela, (Ephyra?) (Venezuela). - Warr., Nov.Zool,, Matto7, p. 165 (1900) (X.). Grosso,

atridiscata Warr., Nov. Zool., 11, p. 33

Bolivien (1904) (Craspedia) (Bolivia) (nom. praeocc., nec. Warr. (1897).

atrisignata Warr., M.S. (Craspedia) (nom. nov., vice atridiscata).

perstrigala Warr., Nov. Zool., 14, p. 221 (1907) Paraguay, (Paradmeta) (Paraguay). Amazonas

(var.),

Süd-Ost-

Brasilien

viridipunctata Warr., Nov. Zool., 7, p. 152 (1900) Süd-Ost(Craspedia) (S. E. Brazil). Brasilien,

mucosaria Schaus, M.S. (Anisodes).

Paraguay

vigoraria E. D. Jones, Proc. Zool. Soc. Lond, Süd-Ost1921, p. 345, t. 3, f. 8 (Anisodes). Brasilien

ab. (?) grisaria Schaus, M.S. (Anisodes).

cantona Schaus, Tr. Amer. Ent. Soc., 27, p. 192 Mexiko (1901) (Cn.).

sticticata Warr., Nov. Zool., 4, p. 434 (1897) (Cra- Paraguay, spedia).

?Bolivien

oenopodiata Guen., Spec. Gén. Lép., 9, p. 509 Brasilien, (1858) (Acidalia) (Brasil.). - Prout, Tr. NordEnt. Soc. Lond., 1910, p. 214 (1910). - Argentinien, Ch. Oberth., Et. Lép. Comp., 12, p. 173, t. Bolivien 401, f. 3450 (1916) (Acidalia).

fissaria Guen., Spec. Gén. Lép., 9, p. 404 (1858) Cayenne, (Cyphopteryx) (Cayenne).

crinita Warr., Proc. U. S. Mus., 30, p. 461

Amazon 139 (1906) (Schistocolpia) (French Guiana).

\section{Genus Hemipferodes Warr.}

Warr., Proc. U. S. Mus., 30, p. 446 (1906) (typus subnigrata Warr.).

camma Druce, Biol. Centr.-Amer., Lep. Het., 2, p. Guatemala 109 , t. 51 , f. 22 (1892) (Eois).

divarieata Warr., Nov. Zool., 4, p. 439 (1897) Costa-Rica (Lipomelia) (Venezuela). - Prout, Ann. bis Mag. Nat. Hist., (8) 6, p. 243 (1910) (Ha- Columbien, malia). - Dyar, Proc. U. S. Mus., 47, p. Venezuela, 233 (1914) (Lipomelia).

J'rinidad 
rotundata Dogn., Mém. Soc. Ent. Belg., 18, p. 164 Venezuela (1911) (Guyane franç.). bis Fr.Guayana

flavida Prout, Ann. Mag. Nat. Hist., (8) 6, p. 242 Ost-Peru (1910) (Hamalia).

curviplena Warr., Nov. Zool., 2, p. 96 (1895) (Lipo- Franz.melia?) (S. E. Brazil).

Guayana,

Brasilien,

?Bolivien

(var.?)

malvina Druce, Biol. Centr.-Amer., Lep. Het., 2, Mexiko, p. 599, t. 99, f. 13 (1899) (Asthena?) (Pa- Panama nama).

nubilala Schaus, Tr. Amer. Ent. Soc., 27, p. 194 Peru (1901) (Lipomelia).

brumneoslicta Warr., Nov. Zool., 14, p. 219 (1907). S.-O.-Peru

subnigrala Warr., Proc. U. S. Mus., 30, p. 446 Columbien 9 (1906) (Fr. Guiana). bis Fr.-

Trinidad

\section{Genus Lipofaxia Prout}

Prout, Nov. Zool., 25, p. 87 (1918) (typus rubicunda Warr.).

rubicunda Warr., Nov. Zool., 12, p. 324 (1905) Columbien (Lipomelia) (Trinidad). - Prout, Nov. Zool., bis Fr.25, p. 87 (1918). - Kaye \& Iamont, Cat Grayana Trin. Lep. Het., p. 108 (1927). u. UnterAmazon

perpulverosa Prout, Nov. Zool., 27, p. 270 (1920) Peru (pr. var.?).

irregularis Prout, Nov. Zool.j 27, p. 269 (1920) Venezuela, (Maroni).

Fr.-

Guayana,

Unter-

Amazon

rolundala Schaus, Tr. Amer. Ent. Soc., 27, p. 256 Venezuela (1901) (Haemalea).

segmentata Warr., Nov. Zool., 14, p. 220 (1907) Ost-Peru (Lipomelia). - Prout, Nov. Zool., 25, p. 87 (1918) (Lipotaxia?).

subvestita Prout, 1. c. (1918).

6

\section{Genus Trygodes Guen.}

Guen., Spec. Gén. Lép., 9, p. 426 (1858) (typus musivaria H.-Sch.). - Walk., List Lep. Ins., 22, p. 655 (1861).

physciata C. Felder, Reise Novara, Lep. Het., 2, t. Amazonen128 , f. 8 (1875). 
basisignata Prout, Nov. Zool., 24, p. 385 (1917). Süd-OstPeru

spoliataria Möschl., Verh. Zool.-Bot. Ges. Wien, 31, Columbien p. 407 , t. 17 , f. 18 (1881) (Surinam). - bis ? Druce, Biol. Centr.-Amer., Lep. Het., 2, Surinam, p. 106 (1892) (Chiriqui).

Amazonas, Ost-Peru (var.?)

c olumbaris Butl., Tr. Fint. Soc. Lond., 1881 : 3, p. 347 (1881, Sept.) (Eutropa?).

niobe Drume Biol Centr-Amer (Amazon) 106, t. 51, f. 9, 10 (1892) (Panama). Panama,

Peru

var. de sol a ta Prout, Nov. Zool., 24, p. 387 Nord(1917).

Venezuela

amphion Schaus, Ann. Mag. Nat. Hist., (8) 9, p. Costa-Rica 429 (1912).

viridiplena Prout, Nov. Zool., 24, p. 386 (1917) Panama (Venezuela).

m us ivaria Druce, Biol. Centr.-Amer., Lep. Het., 2, p. 105 , t. 51 , f. 6,7 (1892). -Thering, Borbol.-Marip.-Tras. (Bol. $\Lambda$ gric. Sao Paulo, no. $7 / 8$ ), p. 16 , t. 2 , f. 10 (1930) (err. det.).'

musivaria H.-Sch., Samml. Außereur. Schmett., 1 : Columbien, $13 / 17$, t. [61], f. 340 (1855); Schlußheft, p. 62 (1858) (Geometra) (Brasilien). - Guen., Spec. Gén. Lép., 9, p. 426 (1858). - Walk., List Lep. Ins., 22, p. 656 (1861). - Hmpsn., Faun. Ind. Moths, 3, p. 460 (1895) (muscivaria). - Ch. Oberth., Et. Lép. Comp., 12, p. 162 (part.) (1916). - Kaye \& Lamont, Cat. Trin. Lep. Het., p. 109 (1927).

herbiferata Guen., Spec. Gén. Lép., 9, p. 426 , t. 15, f. 4 (1858). - Walk., List Lep. Ins., 22, p. 655 (1861). - Mabilde, Guia Prat. Coll. Ins., p. 225, t. 23 , f. 10 (1896). - Ch. Oberth., E.t. Lép. Comp., 12, p. 162 (1916).

B i o log ie: Mabilde, 1. c. (1896).

solaniferata Guen., Spec. Gén. Lép., 9, p. 427 (1858) - Walk., List Lep. Ins., 22, p. 656 (1861). - Ch. Oberth., Et. Lép. Comp., 12, p. 162 t. 398, f. 3383 (1916) (praec. form.?).

? var. herbida Oh. Oberth., 1. c., f. 3384 (1916).

merla Druce, Biol. Centr.-Amer., Iep. Het., 2, p. Mexiko, 106 , t. 51, f. 11 (1892) (Guatemala). - Guatemala, Dyar, Proc. U. S. Mius., 47, p. 232 (1914). Honduras, - Ch. Oberth., Et. Lép. Comp., 12, p. 162 Columbien (1916) (praec. syn.?).

Brasil.-

Amazon

Venezuela, Trinidad, Paraguay, Brasilien 
dissuasa Prout, Nov. Zool., 27, p. 270 (1920).

West-

Ecuador,

? Bolivien

simplicissima Dyar, Proc. U. S. Mus., 42, p. 88 Mexiko 11

(1912) (huj. gen.?).

\section{Genus Neothysanis Dogn.}

Dogn., Hét. Nouv. Amér. Sud, 9, p. 19 (1916) (typus bicolor Dogn.).

aloxogramma Prout, Nov. Zool, 37 : 2, p. 231 (1932). Bolivien

imella Druce, Biol. Centr.-Amer., Lep. Het., 2, p. Guatemala, 540 , t. 99 , f. 18 (1899) (Erosia?) (Guate- Fr.mala). - Prout, Nov. Zool., $37: 2$, p. 231 Guayana, (1932).

Süd-Peru,

Bolivien,

Brasilien

bicolor Dogn., Ann. Soc. Ent. Belg., 44, p. 444 Ecuador (1900) (Erythrolophus); id., Hét. Nouv.

3 Ámér. Sud, 9, p. 19 (1916).

\section{Genus Proufoscia Schaus}

Schaus, Ann. Mag. Nat. Hist., (8) 10, p. 286 (1912).

mirifiea Schaus, Ann. Mag. Nat. Hist., (8) 10, p. Costa-Rica 1 206 (1912).

\section{Genus Asellodes Guen.}

Guen., Spec. Gén. Lép., 9, p. 424 (1858). - Walk., List Lep. Ins., 22, p. 654 (1861). - Warr., Nov. Zool., 7, p. 148 (1900). - Schaus, Tr. Amer. Ent. Soc., 27, p. 194 (1901). - Prout, Tr. Ent. Soc. Lond., 1910, p. 213 (1910) (typus laternaria Guen.).

Pseudasellodes Warr., Nov. Zool., 11, p. 39 (1904) (typus constellata Warr.).

Morphologie: Warr., l. c. (1900). - Prout, 1. c. (1910).

constellata Warr., Nov. Zool., 11, p. 40 (1911) Peru (Pseudasellodes).

d a pal is Ch. Oberth., Et. Lép. Comp., 12, p. 161, t. 397, f. 3381 (1916).

lacunata Dogn., Ann. Soc. Ent. Belg., 50, p. 109 Nord(1906) (Pseudasellodes) (Rép. Argentine). Argentinien,

daphnogethes Ch. Oberth., Et. Lép. Paraguay Comp., 12 , p. 161,162 , t. 398 , f. 3382 (1916) (Paraguay). 
schadei Schaus (M.S.) (Hydratoscia) (Paraguay).

cassiopeia Bastelb., Ent. Zeits. Stuttgart, 22, p. 59 Nord(1908) (Pseudasellodes) (pr. f.?). Argentinien

vitraria Schaus, Tr. Amer. Ent. Soc., 27, p. 194 Ecuador (1901) (Ecuador).

Bolivien

lhebetior Warr., Proc. U. S. Mus., 30, p. 432 (1906). Fr.-

d a u lias Ch. Oberth., Et. Lép. Comp., 12, p. 161 , t. 397 , f. 3380 (1916).

laternaria Guen., Spec. Gén. Lép., 9, p. 425, t. 21, Panama, f. 3 (1858) (Brésil). - Walk., List Lep. TabogaIns., 22, p. 654 (1861). - Sand, Monde des Insel, Pap., p. 124 (1867). - Druce, Biol. Centr.- Trinidad, Amer., Lep. Het., 2, p. 105, t. 51, f. 8 Venezuela, (1892). - Prout, Tr. Ent. Soc. Lond,, 1910, Bolivien, p. 213 (1910). - Kaye \& Lamont, Cat. Trin. Argentinien, Lep. Het., p. 109 (1927). Brasilien

platygymna Prout, Nov. Zool., $37: 2$, p. 231 (1932). Bolivien

nigrofasciaria H.-Sch., Corr.-Bl. Zool.-Min. Ver. Re- Cuba gensb., 24, p. 183 (1870). - Gdl., Fint. Cubana, 1, p. 389 (1881).

fenestraria (Zell., M.S.) Guen., Spec. Gén. Lép., 9, Cuba, p. 425 (1858) (Colombie?). - Walk., List Trinidad, Lep. Ins., 22, p. 655 (1861). - H.-Sch., Mexiko, Cor.-Bl. Zool.-Min. Ver. Regensb., 24, p. Costa-Rica, 183 (1870). - Gdl., Ent. Cubana, 1, p. Süd388 (1881). - Druce, Biol. Centr.-Amer., Amerika Iep. Het., 2, p. 105 (1892), p. 538 (1899). - bis SüdDogn., Lép. Loja, 2, p. 42 (1891). - Oh. Brasilien Oberth., Et. Lép. Comp., 12, p. 160, t. 397, f. 3877,3878 (1916).

O thyreat a C. Felder, Reise Novara, Lep. Het., 2, t. 128, f. 6 (1875) (Venezuela). Möschl., Verh. Zool.-Bot. Ges. Wien, 31, p. 487 (1881).

da $p h n$ ite $s$ Ch. Oberth., Et. Lép. Comp., 12, 9 p. 160, t. 397, f. 3379 (1916) (Peru).

\section{Genus Synegiodes Swinh.}

Swinh., Tr. Ent. Soc. Lond., 1892, p. 11, fig. (1892) (excl. cit. typ.). Hampson, Faun. Ind., Moths, 3, p. 453 (Erythrolophus

Synegioides Swinh., Ann. Mag. Nat. Hist., (6) 12, p. 150 (1893) (typus sanguinaria Moore).

Morphologie: Swinh., Tr. Ent. Soc. Lond., 1892, p. 11. - Prout, Nov. Zool., 25, p. 78 (1918). 
hyriaria Walk., List Lop. Ins., 35, p. 1617 (1866) (Anisodes) (N. Hindostan). - Moore, Proc. Zool. Soc. Lond., 1867, p. 641 (1867). Butl., Ill. Het., 6, p. 75, t. 118, f. 5 (1886) (Anisodes). - Swinh., Cat. Lep. Het. Oxf. Mus,, 2, p. 371 (1900). - Hampson, Proc. Zool. Soc. Lond., 1906, p. 492 (Erythrolophus). - Prout, Nov. Zool., 25, p. 78. (1918); id., Journ. Bomb. Nat. Hist. Soc., $31: 1$, p. 136 (1926).

hyriarius Hampson, Faun. Ind, Moths, 3, p. 453 (1895) (Erythrolophus). - Longstaff, Butt.-Hunting, p. 77 (1912) (Erythrolophus).

obliquifascia Prout, Nov. Zool., 25, p. 78 (1918) Nond-Ost(Khasiberge).

Himalaya

diffusilascia Swinh., Tr. Int. Soc. Lond., 1892, p. Sikkim, 11 , t. 1, f. 9 (1892); 1894, p. 180 (1894) Assam, (Khasiberge). - Hampson, Faun. Ind., Tonkin Moths, 3, p. 454 (1895) (Erythrolophus). Prout, Nov. Zool., 25, p. 78 (1918). - J. Joann., Ann. Soc. Ent. Fr., 98, p. 477 (1929).

diffusaria Swinh., Tr. Ent. Soc. Iond., 1892, p. 11 (1892) (crr. det.).

histrionaria Swinh., Tr. Ent. Soc. Lond,, 1892, p. Sikkim, 12 (1892) (Khasiberge), 1894, p. 180 (1894); id., Cat. Lep. Het. Oxf. Mus., 2, p. 371 (1900). - Hampson, Fuun. Ind., Moths, 3, p. 453 (1895) (hyriarius var.). - Prout, Nov. Zool., 25, p. 78 (1918). - J. Joann., Ann. Soc. Int. Fr., 98, p. 476 (1929).

var. o r n a ta Bastelb., Ent. Zeits. (Stuttg.), 23 , p. 34 (Spilopera), p. 39 (1909); id., Iris, 22, p. 174 (1909). - Prout, Ent. Mitt., 3, p. 240 (1914).

lentiginosaria Wileman, Entom., 44, p. 401 (1911) (Anisodes) (omata syn.).

sanguinaria Moore, Proc. Zool. Soc. Lond., 1867, p. Sikkim, 641 (1867) (Anisodes) (Sikkim). - Swinl., Naga Tr. Int. Soc. Lond., 1892, p. 12 (1892); id., Ann. Mag. Nat. Hist., (6) 12, p. 150 (1893) (Synegiodes); id., Cat. Lep. Het. Oxf. Mus., 2, p. 365 (1900) (Chrysolene). - Hampson, Faun. Ind., MLoths, 3, p. 442 (1895) (Acidalia). - Prout, Nov. Zool., 25, p. 78 (1918).

brunnearia Leech, Ann. Mag. Nat. Hist., (6) 20 , p. West107 (1897) (Ephyra). - Prout in Seitz, Ma- China crolep., 4, p. 46, t. 5, f. (1913) (Anisephyra). - Sterneck, Iris, $41: 2$, p. 154 (1927) (Anisephyra) (huj. gen.?).

Morphologie: Sterneck, l. c. (1927). 6 


\section{Genus Anisephyra Warr.}

Warr.s Nov. Zool., 3, p. 369 (1896) (typus rufaria Warr.). Prout in Seitz, Macrolep., 4, p. 46 (1913).

rufaria Warr., Nor. Zool., 3, p. 370 (1896). - Rajputana Hampson, Journ. Bomb. Nat. Hist. Soc., $12: 1$, p. 85 (1898) (Ephyra).

ocularia F., Syst. Ent., p. 625 (1775); id., Spec. Ins., Ceylon, 2, p. 248 (1781); id., Mant. Ins., 2, p. 190 Indien bis (1787); id., Ent. Syst., $3: 2$, p. 146 (1794) Formosa (India). - Prout, Bull. Hill Mus., 4:1, p. 129 (1930).

a $l b a n n u l a r i a$ Walk., List Lep. Ins., 22, p. 554 (1861) (Thabassodes). - Moore, Lep. Ceyl., 3, p. 429 (1887) (Thalera). - Hampson, Faun. Ind., Moths, 4, p. 563 (1896) (Ephyra). - Swinh., Cat. Lep. Het. Oxf. Mus., 2, p. 366 (1900); id., Tr. Ent. Soc. Lond., 1902, p. 662 (1902).

albiannularia Warr., Nov. Zool., 3, p. 369 (1896).

inve xa ta Walk., List Lep. Ins., 26, p. 1497 (1862) (Epione?). - Swinh., Proc. Zool. Soc. Lond., 1885, p. 856 (1885) (Ephyra); id.; 1. c., 1886, p. 455 (1886) (Ephyra inexacta [err. typogr.]). - Moore, Lep. Ceyl., 3, p. 442, t. 199, f. 2 (1887) (Cyclophora). - Hampson, Ill. Het., 9, p. 39 (1893); id., Faun. Ind., Moths, 3, p. 445 (1895) (Ephyra). monochromata Walk., List Lep. Ins., 26, p. 1754 (1862) (Ephyra). Moore, (India) Proc. Zool. Soc. Lond., 1866, p. 365 (1866) (Ephyra).

ab. maculifascia Hampson, Mll. Het., 8, p. 111, t. 151, f. 9 (1891); id., Faun. Ind., (Nilgiris) Moths, 3, p. 445 (1895) (Ephyra).

ab. qu i e ta Swinh., Proc. Zool. Soc. Lond., 1885 , p. 856 , t. 56, f. 1 (1885) (Ephyra). - (Bombay) Moore, Lep. Ceyl., 3, p. 443 (Cyclophora). Hampson, 1. c. (1895) (Ephyra).

var. incorrupta Warx., Nov. Zool., 3, p. Java bis 370 (1896) (Timor). Timor

var. (ab.?) a urata Warr., Nov. Zool., 4, Kalao 2 p. 47 (1897).

\section{Genus Palaeaspilates Warr.}

Warr., Nov. Zool., 1, p. 370 (1894); id., 1. c., 5, p. 10 (1898); id., Ann. S. Afr. Mus., 10:12, p. 484 (1914). - Prout, Gen. Ins., 104, p. 4, 38 (1910); id. in Seitz, Macrolep., 16, p. 49 (1933). 
inoffensa Warr., Nov. Zool., 1, p. 370 (1894) (Knys- Kapland, na). - Prout in Seitz, Macrolep., 16, t. 6 a Natal (1933).

ab. $r$ u $b_{i}$ d $a$ Warr., Ann. S. Afr. Mus., $10: 12$, p. 484 (1914).

mansueta Prout, Ann. S. Afr. Mus., 17, p. 50 (1917); Buschmann19, t. 16, f. 5 (1925); id. in Seitz, Macrolep., Land 2 16 , p. 50 , t. 6 a $(1933)$.

\section{Genus Haemafopis $\mathrm{Hb}$.}

Hb. (Zutr. Exot. Schmett., 2, p. 26, indescr.); id., Torz. Bek. Schmett., p. 301 (1825) (typus grataria F.). - Guen., Spec. Gén. Lép., 10, p. 170 (1858). - Pack., Mon. Geom. U. S., p. 200, 218; t. 2, f. 6 (1876). - Gmpbg., Nova Acta Acad. Leop. Nat., 49 : 4, p. 333 (1887); $58: 4$, p. 297 (1892).

II a ematopsis Hulst, Tr. Amer. Ent. Soc., 23, p. 295 (1896). - Dyar, List N. Amer. Lep., p. 290 (1902). Mosher, Bull. Ill. Lab. Nat. Hist., 12, p. 126, 127, 129, 131 (1916).

grataria (Weber, Nomencl. Ent., p. 132, 1795, nom. Nordnud.), F., Supp. Ent. Syst., p. 453 (1798). Amerika - Guen., Spec. Gén. Lép., 10, p. 171, t. 19, f. 6 (1858). - Walk., List Lep. Ins., 24, p. 1054 (1862). - Riley, Rep. Ins. Missouri, 1, p. 179 , t. 2, f. 18-21 (1869). - Pack:; Mon. Geom. U. S., p. 219, t. 9, 1. 41 (1876). - Bowles, Can. Ent., 15, p. 165 (1883). Gmpbg., Nova Aeta Acad. Leop. Nat., 58 : 4, p. 297 (1892); $65: 3$, t. 15 , f. 103 (1896). Comst., Man. Stud. Ins, p. 287, f. 342 (1895). - Hulst, Tr. Amer. Ent. Soc., 23, p. 295 (1896) (Ilaematopsis). - Denton, Moths \& Butts. U. S., 1, p. 53, fig. (1899). - Barnes \& Mrc Dunnough, Check List Lep. Bor. Amer., p. $103(1917)$.

s a n ia ria Hb., Zutr. Exot. Schmett., 2, p. 26, f. 345,346 (1822); id., Verz. Bek. Schmett., p. 301 (1825). - H.-Sch., Samml. AuBereur. Schmett., $1: 18 / 22$, p. 34 (1856) (Timandra) (Surinam [err. loc.]).

successaria Walk., Can. Nat., 5, p. 262

(1860) (Pellonia); id., List Brit. Ins., 26, (Montreal)

p. 1617 (1862) (Rhodostrophia).

ab. annettearia Haimbach, Ent. Nows, 18, p. 45 (1907) (Haematopsis) (Ohio).

Bi ol og i e: Riley, l. c. (1869). - Pack., l. c. (1876). - Dyar, Psyche, 9, p. 155 (1901). F. M. Jones, Trans. Ent. Soc. Lond., $80: 2$, 1 p. 352 , t. 18 , f. 19 (1932). 


\section{Genus Calofhysanis $\mathrm{Hb}$.}

Hb., Verz. Bek. Schmett., p. 301 (1825) (typus amataria L.). Pack., Mon. Geom. U. S., p. 316 (1876). - Rghfr. in Beck, Faun. Hernstein, 2:2, p. 37 (1885). - Meyr., Tr. Ent. Soe. Lond., 1892, p. 91 (1892). - Hulst, Tr. Amer. Ent. Soc., 27, p. 297 (1896). Prout, Tr. City Lond. Ent. Soc., 20, p. 24 (1911).

T i mandra Dup., Hist. Nat. Lép. Eur., 7 : 2, p. 224 (1829); id., Cat. Méth., p. 223 (part.) (tvpus amataria L.). - Bsd., Gen. et Ind. Meth., p. 227 (1840). - H.-Sch., Samml. Außereur. Schmett, $1: 18 / 22$, p. 35 (1856). - Heinem., Schmett. Deutschl., 1, p. 706 (1859). - Walk., List Lop. Ins., 23, p. 797 (1861). - Snell., Vlind. Ned, Macrolep., p. 567 (1867). - Fritsch, Denks. Akad. Wien, Math,-Nat. Kl., 41 : 1, p. 94 (1881) (Phänologie). - Poppius, Acta Soc. F. F. Fenn., $3: 3$, p. 119 (1887). - V. Ström, Danm. Sommerf., p. 337 (1891). - Korb, Schmett. Mittel-Eur., p. 177 (1894). - Kappel \& W. E. Kirb., Br. \& Eur. Butt. \& Moths, p. 188 (1895). - Hampson, Faun. Ind., Moths, 3, p. 458 (sect. 1) (1895). - Gmpbg., Nova Act. Acad. Leop. Nat., $65: 3$, p. 315 (1896). - Oudemans, Ned. Ins., p. 284 (1900). - Reb., Berge's Schmetterlingsb. (ed. 9), p. 327 (1910). - Prout in Seitz, Macrolep., 4, p. 47 (1913). Blaschke, Raupen Europas, p. 132, 201 (1914). - Eckstein, Schmett. Deutschl., 4, p. 14, t. 49, f. 7 (1923).

B radye petes Steph. (Nom. Brit. Ins., p. 44, 1829 et Cat., 2, p. 128, 1829, indescr.); id., Ill. Haust., 3, p. 201 (1831) (typus anataria L.). - Westw., Synops. Gen. Br. Ins., p. 101 (1840). - Staint., Man. Brit. Butt. \& Moths, 2, p. 50 (1858). - Barrett, Lep. Brit. Isl., 8, p. 81 (1901).

B radypetes Zell. in Agassiz, Nomen. Zool. Index Univ., p. $42(1846)$.

E rastria (Hb. Tent., p. 2, ined., Lep. Zutr., p. 12, 1820, indescr.) Moore, Lep. Ceyl., 3, p. 457 (1887). - Grote, Allg. Zeits. Ent., $7: 22$, p. 470 (1902). - Hulst in Dyar, List N. Amer. Lep., p. 290 (1902).

comae A. Schmidt, Int. Ent. Zoits., $25: 6$, p. 57, Südfig. (1931) (T. amata form., sp. n.?) (sequ. Spanien f.?).

amata L., Syst. Nat. (ed. 10), 1. p. 524 (1758) (Eu- Europa, ropa). - . Gleditsch, Forstwissensch., 1, p. 519 Kleinasien (1775). - Stdgr., Cat. Lep. Eur. (ed. 2), bis Altai, p. 153 (1871); (ed. 3), 1, p. 279 (1901); Nordid., Iris, 10, p. 23 (1897). - Ramann, Afrika Schmett. Deutschl., 4, p. 37 , t. 56, f. 44 (1876) (T.). - Frey, Lep. Schweiz, p. 198 (1880) (T.). - Poppius, Acta Soc. I. F. Fenn., $3: 3$, p. 120 (1887) (T.). - Ch. Auriv., Nord. Fjär., p. 206 (1891) (T.). - Garbsk., Jahresb. Akad. Wien, Math.-Nat. Cl., 101 : 1, p. 111 (1802). - Meyr., Tr. Ent. Soc. Lond., 1892, p. 91 (1892); id., Handb. Brit. Lep., p. 246 (1895); id., Rev. Handb., p. 208 (1927). - Gmpbg., Nova Acta Acad. Leop. Nat., $56: 3$, p. 316 , t. 18 , f. 262 (1896) (T.). - Cholodkovsky, E. Hofm. Atlas Babochek Evr., p. 244, t. 58, f. 26 (1897) (T.). 
- Püng., Iris, $10: 2$, p. 365 (1898) (T.). Reichert, Groß-Schm. Leipzig (ed. 3), p. 57 (1900) (T.). - Bartel, Iris, $15: 2$, p. 217 (1903) (T.). - Reb. in Spuler, Schmett. Eur. (E. Hofm., Großschmett., ed. 3), 2, p. 29, t. 58, f. 26 (1904); id., Berge's Schmetterlingsb. (ed. 9), p. 327, t. 40 , f. $11 \mathrm{a}-\mathrm{c}$ (1910) (T.). - Aigner-Abafi, Ann. Mus. Hung., 4, p. 524 (1906) (T.). - Lameere, Man. Faun. Belg., 3, p. 704, 708, f. 3 (1907) (T.). - South, Moths Brit. Isl., 2, p. 134, t. 50, f. 9, 10 (1908) (T.). - Krulikowsky, Lep. Wiatka, p. 142 (1908) (T.). - Slevogt, Arb. Nat. Ver. Riga (n. s.), 12 , p. 172 (1910) (T.). - Ribbe, Lep. Andal. (Iris 23), p. 312 (1912) (T.). - Vorbrodt \& Müll.-Rutz, Schmett. Schweiz, 2:1, p. 33 (1913) (T.). - Prout in Seitz, Macrolep., 4, p. 47 (1913) (T.). - Hellweger, Jahresb. Vicent. Brixell, 39, p. 11 (1914) (T.). Klöcker, Dunm. Faun., 17, p. 24, t. 2, f. 41 (1915) (T.). - Hoffmann \& Klos, Mitt. Nat. Ver. Steiermark, 53, p. 84 (1917) (T.). - Culot, Noct. Géom. Eur., 3, p. 99 (1918) (T.). - Strand, Archiv f. Naturg. $85 A: 4$, p. 5 (1919) (T!). - Strand, Int. Ent. Zeitschr., 15, p. 37 (1921) (T.). - Stephan, Iris, 39, p. 72 (1925) (T.). - Dannehl, Ent. Zeits. (Frankf.), $40: 21$, p. 460 (1927) (T.). a m a ta ria L., Faun. Suec., (ed. 2), p. 322 (1761); id., Syst. Nat., (ed. 12), 1 : 2, p. 867 (1767). - Müll., Faun. Fridr., p. 47 (1764); id., Zool. Dan. Prodr., p. 124 (1776). - F., Syst. Ent., p. 621 (1775); id., Mant. Ins., 2, p. 185 (1787); id., Ent. Syst., $3: 2$, p. $132(1794)$. - [Schiff.] Schmett. Wien., p. 103 (1775). - Rott., Naturf., 11, p. 68 (1777). - Göze, Ent. Beytr., 3:3, p. 281 (1781). - Knoch, Beytr. Ins., 3, p. 13, t. 1, f. 10, 11 (1783). - De Villers, Linn. Ent., 2, p. 292 (1789). - Rossi, Faun. Etrusc, 2, p. 191 (1790); (ed. Ill.), 2, p. 306 (1808). Hb., Beitr. Gesch. Schmett., 2, p. 191 (1791); id., Samml. Eur. Schmett., Geom., t. 10, f. 52 (1798); id., Tent., p. [2] (ined.) (Erastria); id., Lep. Zutr., p. 12 (1820) (Erastrin); id., Verz. Bek. Schmett., p. 301 (1825). - Schwarz, Kleem. Beytr., 2:1, p. 24, t. 3, f. 6, 7 (1793). - Bikh., Naturg. Eur. Schmett., 5, p. 118 (1794). - Esp, Schmett. in Abbild., $5: 7$, p. 47, t. 7, f. 3-6 (1795). — Schrank, Faun. Boica, 2 :2, p. 15 (1802). - Tisch., Taschenb. Schmett., p. 119 (1804). - Scharfenberg in Bchst. \& Scharfenb., Schädl. Forstins., 3, p. 666 (1805). - Latr., Hist. Nat. Crust. Ins., 14, p. 223 (1805). - Haw., Lep. Brit., 2, p. 296 (1809) (Geometra). - Tr., Schmett. Eur., 5:2, p. 427 (1825); 6:1, p. 20 
(1827) (Ennomos). - Lep. de St. F. \& Serv. in Encycl. Méth., 10, p. 92 (1825) (Phalaena). - Meig., Handb. Schinett., 194, t. 14, f. 4 (1825) (Geometra). - Freyer, Beitr. Gesch. Eur. Schmett., $2: 10$, p. 35 t. 60 , f. 1 (1828); id., Isis, 1842, p. 45,46 (1842) (Ennomos). - Dup., Hist. Nat. Lép. Eur., $7: 2$, p. 105, 226, t. 148, f. 3 (1829); id., Cat. Méth. Lép. Tur., p. 223 (1845) (T.). - Steph., Syst. Cat. Brit. Ins,, 2, p. 128 (1829); id., Ill. Haust., 3, p. 202 (1831) (B.). - De Haan in Lyonet, Anat. (Mém. Mus. Hist. Nat. Paris, 19), p. 454, t. 26, f. 12-17 (1830) (Ennomos); id., Rech. Anat. Ins., p. 565 (1832). - Wood, Index Ent., p. 88 , t. 20 , f. 533 (1835) (Phalaena). Thon, In- u. Ausl. Selimett., 178, t. 62, f. 921 (1837) (Ennomos). - H.-Sch. in Panz., Deutsclil. Ins., 172, p. 100 (1840) (Idaea); id., Syst. Bearb. Sehmett. Eur., 3: 18, p. 13 (1846) (Acidalia). - Westw. in Humphr. \& Westw., Brit. Moths, 2, p. 27, t.62, f. 1, 2 (1843?) (B.). - Bertol.g Nov. Comment. Bonon., 9, p. 135 (1849) (Ennomos). Led., Verh. Zool.-Bot. Ver. Wien, 3, p. 175 (sep. p. 11) (1853) (T.1. - Ad, Spr., Deutsch. Schmettkunde., p. 192 (1856) (T.). - Kayser, Deutschl. Schmett., p. 478, t. 139 , f. 1 (1857) (T.). - Staint., Ent. Weokl. Int. 2, p. 113, 114, fig. (1857); Man., 2, p. 50, fig. (1858); id., Brit. Butt. \& Moths, p. 219 , t. 110, f. 5 (1867) (B.). - Guen., Spec. Gén. Lép., 10, p. 2 (1858) (T.). - Crewe, Ent. Weekl. Int., †, p. 60 (1859) (T.). Heinem., Schmett. Deutschl. 1, p. 707 (T.). - Zebrawski, Motyl. Krakowa, p. 169, t. 10, f. 74 (1860) (Ennomos). - Walk., List Lep. Ins., 23, p. 797 (1861) (T.). - Stdgr., Cat. Lep. Eur. (ed. 1), p. 67 (1861) (T.) Peyrhft., Cat. Lép. Als., 1, p. 94 (1862); (od. 2) 1, p. 117 (1880) (T. - Snell., Vlind. Ned., Macrolep., p. 565 (1867) (T.).Newm., Brit. Moths, p. 84 (1869) (T.). Beroe, Faun. Ent. Franç. Lép., 5, p. 182, t. 51, f. 8 (1873) (T.). - Barr., Lep. Brit. Isl., 8, p. 81 , t. 335 , f. $2-2$ d (1901) (B.). - Hulme, Butt. \& Moths Countryside, p. 231 t. 24, f. 202 (1903) (T.). - Seitz, Macrolep., 4, t. $5 \mathrm{f}$ (1913) (non binom.). - Culot, Noct. \& Géom. Eur., 3, t. 15, f. 296 (1918).

vibicaria. Hofn., Berl. Mag., 4, p. 514 (nom. praeocc., nec Clerck).

a matoria F., Spec. Ins., 2, p. 242 (1781). - Westw., Synops. Gen. Brit. Ins., p. 101 (1840) (B.). - Ménétr., Bull. Acad. Sci. St. Pét., 17, p. 316 (1858) (T.).

ang u la ta [E. Geoffr.] Foure., Ent. Paris., 2 , p. 270 (1785) (nom. praeocc.). 
a matorio Donov., Nat. Hist. Brit. Ins., 1, p. 74, t. 33 , f. 2 (1793).

ab. effusaria Klemensiewicz, Verh. Zool.Bot. Ges. Wien, 44, p. 184 (1894). - Stdgr. \& Reb., Cat. Lep. Palaearct., 1, p. 279 (1901). - Prout in Seitz, Macrolep., 4, p. 48 (1913).

- (s. n.) De Roo van Westmaas in Sepp, Ned. Ins., (2) 2, p. 231, t. 49, 1. 2 (1872). ab. ros eata Hirshke, Verh. Zool-Bot. Ges. Wien, 60, p. 414 (1910). - Prout, 1. c. (sine loc.) (1913).

ab. nigra (Cupper, Entom., 26, p. 65, 1893, s. n.; Barr., Lep. Brit. Isl., 8, p. 82, t. 335, f. 2 c, 1901, s. n.). Reb., Berge's Schmettcrlingsb. (ed. 9), p. 327 (1910). - Prout, l. c. (1913).

ab. de leta (Hb.-Gey., Samml. Eur. Schmett. Geom., t. 101, f. $524,525,1827$, s. n.) Reb., 1. c. (1910) - Prout, l. c. (1913). Sterneck, Prodr. Schmett. Böhm., p. 162 (1929). ab. $s u f f u m$ at $a$ (Barr., Lep. Brit. Isl., 8, p. 82,1901 , var., s. n.) Prout, 1. c. (1913). ab. b i p a $\boldsymbol{r}_{\text {t }}$ t a (Riedel, Iris, 18, p. 153, t. 2, f. 5, 1905, s. n. - Aigner-Abafi, Ann. Mus. Hung., 4, p. 524, 1906, ab., s. n.) Prouts. 1. c. (1913). - Möbius, Iris, 36, p. 77 (1922).

ab. $l$ a $t$ is $t r i g a$ Reb., Rov. Lapok, 22, p. 174 (1915).

ab. s e re n a ta Dannehl, Fnt. Zeits. (Frankf.), $40: 21$, p. 461 (1927).

var. (?) griseata W. Petersen, Lop. Estl., p. 116 (1902); (ed. 2), 1, p. 231 (1924). grisearia Prout in Seitz, Macrolep., 4, p. 48 (1913).

var. recompta Prout, Nov. Zool., $35: 4$, p. 297 (1930) (Ussuri). - amataria Brem, Mém. Acad. Sci. St. Pét., (7), 8:1 (Lep. Ost-Sib.), p. 79 (1861). — amata Stdgr., Iris, 10, p. 23 (1897). - Lrech, Ann. Mag. Nat. Hist., (6) 20, p. 109 (pirt.) (1897). -comptaria (part.) Prout in Seitz, Macrolep., 4, p. 48 (amata var.) (1913).

I cones: Schaeffer, Ic. Ins. Ratisb., $2: 1$, $t$. 122 , 1. 4 (s. n.); id., 1. c., 3 , t. 214 , f. 3 (s. n.) (1767).

Morphologie: H.-Sch., Syst. Bearb. Schmett. Eur., Umrißtaf., p. VIII, X, t. 8, f. 15 (1844). - Berge, Schmetterlingsb., t. 2, f. 27 (1851). - Led., 1. c., f. 7 (1853). Walter, Palp. Maxill. Lep., p. 29 (1884) (Jena. Zeits. Nat., 18, p. 149, 1885). W. Petersen, Mém. Acad. Sci. St. Pét., (8) 9, p. 96 (1900). - Pierce, Genit. Geom. Brit.,

Baltische

Länder, Rußland (pr. p.)

Ussuri,

Korea,

Japan,

Zentral-

China,

? West-

China 
p. 36, t. 23 (1914). - Ch. Oberth., Et. Lép. Comp., 20, p. 214 (1923) (gynandro.).

Biologie: Merian, Fruc. Ortus, 2, t. 5, 43 (1683). - Ray, Hist. Ins., p. 286 (1710). Knoch', 1. c. (178.3). - Tisch., l. c. (1804). - Hb., Gesch. Eur. Schmett. Larv., 5, Geom., 1, Ampliss. J. b, f. 1 a, b. - Sepp, Besch. Wonder. Gods, 4, p. 85, t. 25 (181021). - Freyer, 1. c. (1828). - Die Haan in Lyonet, 1. c. (1830). - Newm., Entom., 4, p. 95 (1868). - Praun, Abbild. Eur. Schmett.-Raup., t. 1, f. 27 (1875). - Thalenhorst, Verh. Vor. Nat. Hamb., 2, p. 150 (1876). - Ramann, 1. c., t. 1, f. 31 (1876) (ovum). - O. Wilson, Iarv. Brit. Lep., p. 115, t. 21, f. $13(1880)$. - E. Hofm., Raup. Großschmett. Eur., p. 177, t. 40, f. 13 (1893). - Schröder, Entwickel. Raupenzeichn., p. 23,43, f. 20, 35 (1894). Barr. 1. c. (1901). - Frionnet, Chen. Macrolep. Fr., p. 141 (1904). - Meyer, Ins.Börse, 22, p. 99 (1905). - Uffeln, Großschmett. Westfal., p. 112 (1908). - Skala, Int. Ent. Zeits., $3: 42$, p. 225 (1910). - C. Schneider, Int. Ent. Zeits., $17: 20$, p. 159 (1924). - V. G. M. Schultz, Bcitr. Lippisch. Großschmett., p. 19 (1930). - L. P. Robert, Les Chenilles, p. 331, t. 52 (1931).

comptaria Walk., List Lep. Ins., 26, p. 1615 (1862) (T.) (China). - Butl., I1l. Het., 3, p. 40, t. 51, f. 2 (1879) (T.). - Leech, Ann. Mag. Nat. Hist., (6) 20, p. 109 (part.) (1897) (T.) - Prout in Seitz, Macrolep., 4 , p. 48 (1913) (T. amate c.); id., Nov. Zool., 35 : 4, p. 297 (1930). - Sterneck, Iris, $41: 2, \mathrm{p} .155$ (1927) (T. amata c.).

? a mataria Ch. Oborth., Et. Lép. Comp., 20, p. 214 (part.) (1933) (Timandra).

Morphologie: Prout, 1. c. (1930).

responsaria Moore, Lrep. Coll. Atk., p. 255 (1888) (T.) (Cherrapunji). - Swinh., Tr. Ent. Soc. Lond., 1894, p. 177 (1894) (T.). - Hampson, Faun. Ind., Moths, 3, p. 459 (1895); id., Journ. Bomb. Nat. Hist. Soc., $12: 1$, p. 87 (part.) (1898) (T.). - Swinh., Cat. Lop. Het. Oxf. Mus., 2, p. 373 (part.) (1900). J. Joann., Ann. Soc. Ent. Fr., 98, p. 476 (1929) (T.). - Prout, Nov. Zool., 37 (1), p. 18 (1931).

strigulata Warr., Nov. Zool., 3, p. 116 (1896) (T.).

var. a ga no p is Prout, Nov. Zool., 37, (1), p. Süd-Indien 18 (1931) (Malabar). - responsaria Hampson, Tll. Het., 8, p. 33 (1891) (T.).

Bhutan, Assam, Tonkin

Tonkin
(Khasia)

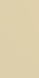

Ussuri,

Japan,

? Formosa

West-

China

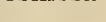


convectaria Walk., List Lep. Ins., 23, p. 800 (1861) (T.) (Silhet). - Moore, Proc. Zool. Soc. Lond., 1867, p. 644 (1867) (T.). - Swinh, Cat. Lep. Het. Oxf. Mus,, 2, p. 373 (1900). Prout in Seitz, Macrolep., 4, p. 48, t. $7 \mathrm{e}$ (1913) (T.); id., Ent. Mitt., 3, p. 240 (1914); id., Nov. Zool., 25, p. 78, 79 (1918) (T.); id., Bull. Hill Mus., $4: 1$, p. $129(1930)$. - J. Joan., Ann. Soc. Ent. Fr., 98, p. 476 (1929) (T.).

c om ptaria Swinh., Tr. Ent. Soc. Iond., 1890, p. 214 (1890) (T.) (err. det.).

a $m$ at aria (part.), Hampson, Faun. Ind., Moths, 3, p. 458 (1895) (T.) (nec L.).

Nelsoni Prout, Nov. Zool., 25, p. 78 (1918) (T.) (S. India).

convectaria Moowe, Lep. Ceyl., 3, p. 457, t. 202, f. 3, 3a. (1887) (Erastria) (urr. det.).

comptaria Hampson, Ill. Het., 8, p. 33 (1891) (T.) (ex'r. det.).

a mataria (part.), Hampson, Faun. Ind., Moths, 3, p. 459 (1895) (T.) (Nilgiris).

oligoscia Prout, Nov. Zool., 25, p. 79 (1918) (T.) (Tibet); id., Journ. Bomb. Nat. Hist. Soc., $31: 1$, p. 136 (1926).

correspondens Hampson, Faun. Ind., Moths, 3, p.
459 (1895) (Dharmsala); id., Proc. Zool. 459 (1895) (Dharmsala); id., Proc. Zool.
Soc. Lond., 1906, p. 492 (1906) (T.). Prout in Seitz, Macrolep., 4, p. 48, t. $7 \mathrm{e}$ (1913) (T.); id., Journ. Bomb. Nat. Hist. Soc., 31 : 1, p. 136 (1926). - J. Joann., Ann. Soc. Ent. Fr., 98, p. 476 (1929) (T.).

commixta Warr., Nov. Zool., 2, p. 100 (1895) (W. Java Java).

a mata var. convictaria (sic) Snell., Tijd. Ent., 25, p. CXXVII (1882) (T.) (err. det.).

punctinervis Prout, Nov. Zool., 23, p. 18 (1916) (T.) (W. Java).

obsoleta Warr., Nov. Zool., 4, p. 63 (1897) (T.). ruptilinea Warr., t. c., p. 64 (1897) (T.) (Khasia).

flavisponsaria Hampson, Journ. Bomb. Nat. Hist. Soc., 21 (4), p. 1248 (1912)' (T.) (Burma).
Nond-

Indien

bis

Tonkin,

Hainan,

Süd- u.

West-

China,

Formosa,

Liu-kiu-

Iuseln,

Luzon

(var.?)

Süd-Indien, Ceylon

Ober-

Birma,

West-

China

Nord-

West-

Indien

bis

Tonkin

Lombok

Java

Malay.-

Halbinsel

(var.?),

? Nord-

Borneo

Khasiberge

Nilghiri-

Berge,

Khasiberge,

Birma 
extremaria Walk., List Lep. Ins., 23, p. 801 (1861) (T.) (,N. Chiua" [Schanghai-Gebiet]). Butl., IIl. Het., 3, p. 40, t. 51, f. 1 (1879) (T.). - Leech, Ann. Mag. Nat. Hist., (6) 20, p. 109 (1897) (T.). - Prout in Seitz,

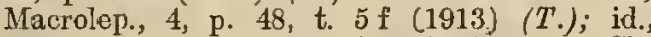
Ent. Mitt., 3, p. 240 (1914) (T.). - Ch. Oberth., Et. Lép. Comp., 20, p. 214 (1923) (Timandra). - Sterneck, Iris, $41: 2$, p. 155 (1927) (T.).

sordidaria Walk., List Lep. Ins., 26, p. 1615 (1862) (T.?) (,N. China").

rectistrigaria Eversm., Bull. Mosc., 24:2, p. 637 Ost(1851) (Ennomos) (E. Siberia). - I Jod., Verh., Zool.-Bot. Ver. Wien, 3, p. 175 (sep. p. 10) (1853) (Acidalia?). - Guen., Spec. Gén. Lép., 9, p. 507 (1858) (Acidalia). Stdgr., Cat. Lep. Eur. (ed. 2), 1, p. 152 (1871); id., 1. c., (ed. 3), 1, p. 279 (1901) (T.). - Erschoff, Bull. Mosc., 43:2, p. 315 (1871) (Acidalia). - Hedem., Hor. Soc. Ent. Ross., 16, p. 243 [bis, recto 259], t. 13, f. 2 b (1881) (T.). - Gmpbg., Nova Acta Acad. Leop. Nat., 54:5, p. 496 '(1890) (Arrhostia). - Meyr., Tr. Ent. Soc. Lond. 1892, p. 91 (1892). - Prout in Seitz, Macrolep., 4, p. 48 (1913) (T.). - Djakonov, Ark. Zool., 21 A : 1, p. 4 (1929); id., Ann. Mus. Zool. URSS., 32, p. 390 (1931) (T.). $P u z i l o i$ Erschoff, Trudy Soc. Ent. Ross., 4, p. 197 (1870) (Irkutzk). - Stdgr., Iris, 10 , p. 23 (1897) (T.). - Gmpbg., Nova Acta Acad. Leop. Nat., 65 : 3, p. 316 (1896) (T.).

Putziloi Graeser, Berl. Ent. Zeits., 32, p. 399 (1888) (T.). - Herz, Iris, $11: 2$, p. 260 (1899) (T.).

ab. obso le ta Prout in Seitz, Macrolep., 4, p. 49 , t. $5 \mathrm{~g}$ (1913). - Djakonov, 1. c. (1929). - puziloi Hedem., Hor. Soc. Fint. Ross., 16, p. 243. [bis, recto 259 ], t. 13, f. $2 \mathrm{a}$ (1881) (rectistrigaria or-f.). - Alph. in Romff., Mém. Lép.; 9, p. 340 (1897) (T.).

amaturaria Walk., List Iep. Ins., 35, p. 1634 (1866) (T.) (N. America). - Pack., Mon. Goom. U. S., p. 317 , t. 10, f. 34 (1876); id. Ins. Life, 4, p. 332 (1892). - Kellicott, Can. Ent., 24, p. 242 (1892). - Gmpbg., Nova Acta Acad. Leop. Nat., 65 : 3, p. 317, t. 17, f. 263 (1896) (T.). - Dyar, List N. Amer. Lep., p. 290 (1902) (Erastria). - Barnes \& Mc Dunnough, Check List Trep. Bor. Amer. p. $103(1917)$ (T.). - Forbes in Jeonand, Ins. New York, p. 592 (1928) (T.).

B i o l o g i e: Kellicott, 1. c. (1892). - Packard, 15 1. c., fig. 76,77 (1892).

Westchina

bis

Formosa

\section{Sibirien,}

Amur,

Kamtschatka
Ostl.-

Vieneinigte-

Staaten 


\section{Genus Plochophyle Warr.}

Warr., Nov. Zool., 3, p. 293 (1896) (typus notata Warr..). - Prout ill Seitz, Macrolep., 4, p. 47 (1913); 16, p. 50 (1933).

Chrysolene Warr., Nov. Zool., 4, p. 4 (1897) (typus deviaria Walk.).

Ptych ophyle Swinh., Tr. Ent. Soc. Lond., 1902, p. 660 (1902) (err. transer.).

marginata Warr., Nov. Zool., 4, p. 392 (1897) (Chry- Khasiberge socraspeda).

pulverulenta Warr., Nov. Zool., 4, p. 60 (1897) Perak (Pt.?).

inornata Warr., Nov. Zool., 3, p. 377 (1896) (Te- Tenimber-, nimber).

Ron-,

s o rdida Warr., Nov. Zool., 6, p. 334 (1899) Luisiaden-, (Dizuga) (Rossel I.).

strigata Warr., Nov. Zool., 9, p. 360 (1902) (err. det.).

Bismarck-

und

sto lida Warr. MS. (Zeugma) (Bougainville). Inseln

exitela Prout, Nov. Zool., 25, p. 79 (1918).

Celebes

laeta (part.) Warr., Nov. Zool., 6, p. 337 (1899) (err. det.).

Pronti Beth.-Baker, Ann. Mag. Nat. Hist., (8) 16, Holl.p. 193 (1915) (Dinawa).

u. Brit.-

Neuguinea

albidlisea Warr., Nov. Zool., 14, p. 145 (1907) Brit.(Zeugma).

Neuguinea

strigata Warr., Nov. Zool., 9, p. 360 (1902) (- Choiseul typus, nec $\left.\sigma^{7}\right)$ (Guadalcanar). bis S.-

Christoval

tristieula Swinh., Proc. Zool. Soc. Lond., 1885, p. Ceylon, 859 , t. 56, f. 17 (1885) (Asthena) (Bom- Indien, bay); id., Tr. Ent. Soc. Lond., 1890, p. 213 Birma, (1890) (Asthena); id., l. c., 1902, p. 660 Luzon, (1902) (Ptychophyle [sic]) (excl. synon.). Sumbawa - Hampson, Faun. Ind., Moths, 3, p. 442 (1895). (Acidalia volutaria form.). - Warr., Nov. Zool., 6, p. 33 (part.) (1899).

volutaria Swinh., Proc. Zool. Soc. Lond., 1885, p. Indien, 858 , t. 56, f. 14 (1886) (Hyria) (Bombay). Malay. - Hampson, Faun. Ind., Moths, 3, p. 442 Halbinsel (1895) (Acidatia). - Warr., Nov. Zool., 3, p. 293 (1896).

virgata West, Nov. Zooloy $35: 3$, p. 254 (1930). Luzon

lineata Warr., Nov. Zool., 3, p. 378 (1896). Timor

nebulifera Prout, Nov. Zool., 27, p. 270 (1920). Brit.- 
innotata Warr., Nov Zool., 3, p. 294 (1896) (volu- Brit. taria subsp.) (Kiriwini). Neuguinea, ab. fasciata Warr', Nov. Zool., 6, p. 33 Trobriand-, (1899) (tristicula ab.) (Goodenough I.). D'Entre-

\section{casteaux- u.}

Luisiaden-

Inseln,

Neu-

Mecklenburg

(var.?)

cyphosticha Turn., Proc. Linn. Soc. N. Sth. Wales, Nord$32: 4$, p. 680 (1908) (N. Queensland); id., Australion, Tr. Roy. Soc. S. Austral., 32, p. 270 (1922) Neuguinea, (Ptychophyle [sic]).

\section{Vulkan-}

Insel

ozophanes Prout, Ann. Mag. Nat. Hist., (9) 1, p. Perak, 22 (1918) (Perak).

Borneo

notata Warr., Nov. Zool., 3, p. 294 (1896) (Fer- Holl.- u. gusson I.). -- Swinh., Tr. Ent. Soc. Lond,, Brit.1902, p. 660 (1902) (Ptychophyle).

insolite Warr., Nov. Zool., 6, p. 331 (1899)

Neuguinea, FergussonInsel (Chr.) (Br. New Guinea).

apicirubra Prout, Nov. Zool., 24, p. 433 (1917); Madagaskar id. in Seitz, Macrolep., 16, p. 50, t. 6 b (1933).

orthogramma Prout, Tr. Ent. Soc. Lond., 1925, p. Madagaskar 307 , t. 36 , f. $9(1925)$; id in Seitz, Macrolep., 16 , p. 50 , t. 5 f $(1933)$.

volutisignata Prout, t. c., p. 309, f. 11 (1925); Madagaskar id. in Seitz, l. c, t. $5 \mathrm{~h}$ (1933).

polyniphes Prout, t. c., p. 310, f. 10 (1925); id. in Madagaskar Seitz, l. c., t. 5 h (1933).

peristoecha Prout, t. c., p. 307, f. 9 (1925); id. in Madagaskar Seitz, l. c., t. 5 h $(1933)$.

anthocroea Prout, t. c., p. 308, f. 10 (1925); id. in Madagaskar Seitz, 1. c., t. 5 h (1933).

hilaris Warr., Nov. Zool., 5, p. 242 (1898); Prout ị Niger Seitz, 1. c. (1933).

Dollmani Prout, Nov. Zool., 37 : 2, p. '232 (1932); Nord-W.id. in Seitz, Macrolep., 16, p. 50, t. 5 h Rhodesia (1933).

mubripennis Warr., Nov. Zool., 5, p. 238 (1898) Niger, (Chrysolene) (Congo). - Prout, Nov. Zool., Congo, 37 : 2. p. 232 (1932); id. in Seitz, Macro- Angola lep., 16 , t. 50 (1933).

ab. sanguinol e $n$ ta Warr.; 1. c. (1898). (Niger)

apseogramma Prout, Nov. Zool., $37: 2$, p. 232 Nord-W.(1932); id. in Seitz, Macrolep., 16, p. 50 Rhodesia (1933).

subminiosa Prout, 1. c. (1932); id. in Seitz, 1. c. Nord-W.(1933). 
liyalotypa Prout, l. c. (1932); id. in Seitz, t. c., p. Kivu 51, t. $5 \mathrm{~h}(1933)$.

miniosa Warr., Nov. Zool., 6, p. 332 (1899) (Chr.) Penang, (Penang). - Prout in Seitz, Macrolep., 4, p. Selangor, 47, t. 5 d (1913). - J. Joan., Ann. Sor. Tonkin, Ent. Fr., 98, p. 480 (1929). ? Ost-

China

medioplaga Swinh., Tr. Ent. Soc. Lond., 1902, p. Pulo-Laut 661 (1902) (Chrysocraspeda).

definita Prout, Bull. Hill Mus., 1 (2), p. 259 (1922); Madagaskar (3), p. 624 , t. 25 , 4. 8 (1924); id. in Seitz, Macrolep., 16, p. 51 , t. 6 a (1933).

rubida Swinh., Tr. Ent. Soc. Lond., 1904, p. 501 Madagaskar (1904) (Stegania). - Prout, Tr. Tnt. Soc. Lond., 1925, p. 309 (1925); id. in Seitz, l. c. (1933).

zaplileges Prout, 1. c., t. 36, f. 16 (1025); id. in Madagaskar Seitz, l. c. (1933) (pr. f.?).

doricaria Swinh., Tr. Ent. Soc. Lond., 1904, p. Madagaskar 563 (1904) (Chrysocraspeda). - Prout in Seitz, 1. c. (1933).

gnamptolowa Prout, Tr. Ent. Soc. Lond., 1925, p. Madagaskar 309 , t. 36 , f. 17 (1925); id. in Seitz, l. c., t. $5 \mathrm{~g}(1933)$.

Kenricki Prout, t. c., p. 310 , t. 36, f. 12 (1925); Madagaskar id. in Seitz, l. c., t. $5 \mathrm{~g}$ (1933).

sanguinipuncta Swiuh., Tr. Ent. Soc. Lond., 1902, Borneo p. 661 (Chrysocraspeda). - Prout, Journ. F. M. S. Mus., $17: 1$, p. 51 (1932).

porphyrochlamys Prout, Nov. Zool., $36: 2$, p. 155 Malayische (1931) (Kedah).

Halbinsel

zearia Swinh., Tr. Ent. Soc. Lond., 1904, p. 563 Madagaskar (1904) (Chrysocraspeda). - Prout in Seitz, Macrolep., 16, p. 51 (1933).

oophora Prout, Nov. Zool., 23, p. 19 (1916).

Sarawak

planaria Swinh., Tr. Ent. Soc. Lond., 1904, p. 464 Madagaskar (1901). - Prout in Seitz, Macrolep., 16, p. $51(1933)$ :

eclipsis Prout, Nov. Zool.s $37: 2$, p. 232 (1932); Madagaskar id. in Seitz, 1. c. (1933).

zombensis Prout, 1. c. (1932); id. in Seitz, 1. c., t. Nyasaland $5 \mathrm{~g}$ (1933).

flavipuncla Warr., Nov. Zool., 6, p. 331 (1899) 우 Philippinen (Chrysolene) (Samar). - G. Semp., Reisen Philipp., (2) 6:6, p.632 (1902) (Chrysolene). of Rothschildi West, Nov. Zool., 35:3, p. 256 (1930) (Chrysocraspeda) (nom. praeocc., nec Warr. 1903).

permutans Hampson, Ill. Het.; 8, p. 33, 123, t. 153, Nilghiri- u. f. 17-21 (1891) (Pseudasthena) (Nilgi- Palniris); id., Faun. Ind., Moths, 3, p. 441 (1895) Berge (Acidalia). - Prout, Ann. Mag. Nat. Hist., (9) 1, p. 24 (1918). 
a uricincta Swinh., Tr. Ent. Soc. Lond., 1902, p. 661 (1902) (Chrysocraspeda) (err. det., nee Hampson).

togata (Weber, Nomencl. Ent., p. 132, 1795, n. nud.) Indien, F., Supp. Ent. Syst., p. 454, O (1798). (Tran- Singapore, quebar). - Prout, Ann. Mag. Nat. Hist., Sumatra, (9) 1, p. 24 (1918):

Java

O faganaria Guen., Spec. Gén. Lép., 9, p. 430 (1858) (Hyria) (Brésil [err. loc.]). Ch. Oberth., Et. Lép. Comp., 12, p. 164, t. 398, f. 3390 (1916) (Hyria).

o deviaria Walk., List Lop. Ins., 22, p. 664 (1861) (Hyria) (Hindostan). - Hampson, Ill. Het., 8, p. 32 (1891) (Pseudasthena); id., Faun. Ind., Moths, 3, p. 442 (1895) (Acidalia). - Swinh., Tr. Ent. Soc. Lond., 1894, p. 192 (1894) (Psilocambogia); id., Cat. Lep. Het. Oxf. Mus., 2, p. 365 (1900) (Chrysolene). - Warr s, Nov. Zool., 4, p. 49 (1897) (Chrysolene).

$\sigma^{r} \boldsymbol{r} h$ od $i$ n a ria Walk., List Lep. Ins., 22, p. 666 (1861) (Hyria?) (Ceylon). - Moore, Iep. Ceyl., 3, p. 450 (1887) (Pseudasthena).

q a moenaria Snell., Tijd. Ent., $33: 3$, p. 222 (1890) (Acidalia) (Sumatra); id., 1. c., $38: 4$, p. 208 (1896) (Ac.).

O a uricincta Hampson, Hl. Het., 9, p. 149 , t. 170 , f. 1 (1893) (Iyria) (Ceylon).

O croceomarginata Warr., Nov. Zool., 6, p. 30 (1899) (Chrysocraspeda) (err. det., nec Warr. 1896).:

crypsaurea Bastelb., Jahrb. Nass. Ver. Nat., 61, p. Perak 78 (1908) "(Chrysocraspeda) (huj. gen.?)"

vinosa Prout, Ann. Mag. Nat. Hist., (9) 1, p. 24 Nord(1918).

Borneo

conversata Walk., List Lep. Ins., 22, p. 666 (1861) Borneo (Hyria). - Swinh., Cat. Lep. Het. Oxf. Mus., 2, p. 366 (1900) (Chrysocraspeda).

anisocosma Prout, Ann. Mag. Nat. Hist., (9) 1, p. Malayische 23 (1918).

Halbinsel

dilucida Warr., Nov. Zool., 4, p. 49 (1897) (Chry- Sarawak solene).

rubricata Swinh., Fascic. Malay. Zool., 1, p. 94 Siam (1903) (Chrysocraspeda).

tigrina Meyr., Tr! Ent. Soc. Lond., 1897, p. 72 Süd-Ost(1897) (Heteroctenis). Borneo

dipyramida Prout, Ann. Mag. Nat. Hist., $9: 1$, p. Tenasserim 22 (1918) (sect. Heteroctenis).

dracontias Meyr., Tr. Ent. Soc. Lond,, 1897, p. 72 Süd-Ost54 (1897) (Heteroctenis).

Borneo 


\section{Genus Chrysocraspeda Hampson}

Swinh., Ann. Mag. Nat. Hist.', (6) 12, p. 157, 1893 (indescr.). Hampson, Faun. Ind. Moths, 3, p. 443 (1895) (typus abhadraca Walk.). - Prout in Seitz, Macrolep., 16, p. 51 (1933).

Heteroctenis Prout, Nov. Zool., 23, p. 24 (1916) (nec Meyr:).

gibhosa Warr., Nov. Zool., 3, p. 370 (1896). Assam

praegriseata Warr., Nov. Zool., 14, p. 139 (1907). Brit.-

Neuguinea

flavimacula Prout, Nov. Zool., 23, p, 20 (1916). Holl.-

Neuguinea

eutmeta Prout, t. c., p. 21 (1916).

Holl.-

Neuguinea

perpicta Warr., Nov. Zool., 3, p. 109 (1896). - Khasiberge Hampson, Journ. Bomb. Nat. Hist. Soc., 12 (1), p. 85 (1898).

mitigata Walk., List Lep. Ins., 22, p. 664 (1861) Indien (IIyria) (Hindostan [Moulmein ]). - Hamp- bis Tonkin, son, Faun. Ind., Moths, 3, p. 443 (1895). - Perak, J. Joann., Ann. Soc. Ent. Fr., 98, p. 478 Borneo (1929).

dinawa Beth.-Baker, Ann. Mag. Nat. Hist., (8) 16, Holl.- u. p. 192 (1916) (Brit. Neu-Guinea).

Brit.-

informiplaga Prout, Nov. Zool., 23, p.

Neuguinea 23 (1916) (Dutch New Guinea).

sanguinea Warr., Nov. Zool., 3, p. 110 (1896). - Khasiberge

Hampson, Journ. Bomb. Nat. Hist. Soc., $12: 1$, p. 85 (1898).

conspicuaria Swinh., Ann. Mag. Nat. Hist,, (7) Sikkim, 15 , p. 165 (1905) (Ḱhasia). - Hampson, Assam, Journ. Bomb. Nat. Hist. Soc., $18: 1$, p. Ceylon 50 (1907) (prnec. ab.?; sequ. form.?)

abhadraca Walk., List Lep. Ins., 22, p. 636(1861) Ceylon, (Ephyra) (Ceylon). - Moore, Lep. Ceyl., Indien, 3, p. 442, t. 199, f. 3, 3a (1887) (Cyclo- Sarawak phora). - Frampson, IIl. Het., 8, p. 28 (1891) (Ephyra); id., 1. c., 9, p. 39 (1893) (IIyria); id., Faun. Ind., Moths, 3, p. 443, 444, f. 202 (1895). - Swinh., Ann. Mag. Nat. Hist., (6) 12, p. 157 (1893); id., Tr. Ent. Soc. Loud., 1894, p. 180 (1894); id., Cat. Lep. Het. Oxf. Mus., 2, p. 366 (1900).

Biologie: Moone, l. c. (1887).

callima Beth.-Bak., Ann. Mag. Nat. Hist., (8) 16, Holl.- u. p. 192 (1915) (Brit. New Guinea).

Brit.-

oxyporphyris Prout, Nov. Zool., 23, p. Neuguinea 22 (1916) (Dutch New Guinea). 
olearia Guen., Spec. Gén. Lép., 9, p. 430 (1858) Indien, (Hyria) (Nord de l'Inde). - Hampson, Hainan, Faun. Ind., Moth/s, 3, p. 444 (1895). - Ch. Oberth., Et. Lép. Comp., 12, p. 165, t. 398, f. 3391 (1916) (Hyria). - Prout, Bull. Hill Mus., $4: 1$, p. 129 (1930).

olenaria Walk., List Lep. Ins., 22, p. 664 (1861) (Hyria) (err. typogr.).

cerasina Swinh., Ann. Mag. Nat. Hist., (6), 12, p. 157 (1893); id., Cat. Lep. Het: Oxf. Musw 2, p. 366 (1900) I("Ceylon" [Ganjam, sec. specim. typ.]).

? croceomarginata Oo Warr., Nov. Zool., 6 , p. 30 (1899) (nec Warr. 1896).

croceomarginata Warr., Nov. Zool., 3, p. $370(1896)$ (S. Java). - ? J. Joann., Ann. Soc. Ent. Fr., 98, p. 478 (1929) (vix praec. var.?)d

? Sumbawa

Java,

Malayische

Halbinsel,

? Tonkin

rosina Warr., Nov. Zool., 5, p. 238 (1898) - - Prout Niger in Seitz, Macrolep., 16, p. 51 , t. 6 b (1933).

var. hete ror a Prout, Nov. Zool., 37:2, p. 233 (1932); id. in Seitz, 1. c. (1933).

Franz.-

Guinea

Ieigliala Warr., Nov, Zool., 11, p. 466 (1904). - Natal Prout in Seitz, 1. cl, t. 6 b (1933).

ab. dehonorat a Prout, Ann. Transv. Mus., 3, p. 196 , t. 12 , f. 13 (1913); id. in Seitz, t. c., p. 52 (1933).

var. holobapta Prout, Nov. Zool., 24, p. Congo, 433 (1917) (Congo); id. in Seitz, t. c., p. Nandi 51 (1933). - medjaria Holland, Bull. Amer. Mus. Nat, Hist., 43 , p. 312 , f. 6 (1920) (Acidalia?) (Congo).

nigribasalis Warr., Nov. Zool., 16, p. 112 (1909). Natal - Prout in Seitz, t. c., p. 52 (1933) (praec. ab.?).

erylluraria Mab., Ann. Soc. Ent. Belg., 27, p. 62 Madagaskar, (1883) (Amaurinia) (Madagascar). - Prout Comoroin Seitz, 1. c., t. 6 b (1933).

rosacea A. Pagenst. in Voeltzkow, Reise in Inseln Ostafrika, $2: 2$, p. 100 , t. 6, f. 9 (1907) (Comoro-Insèln).

nigrop un ctata A. Pagenst. (ex err.), t. c., Aufiki., t. 6, f. 9 (1907).

Heringi Prout, Nov. Zoold, $37: 2$, p. 233 (1932); Südid. in Seitz, 1. c., t. $5 \mathrm{~g}$ (1933). Kamerun

complaria Swinh., Tr. Ent. Soc. Lond., 1902, p. Malayische 662 (1902) (Singapore). Halbinsel, Luzon

var. pulverimargo Prout, Nov. Zool., 24, Brit.p. 313 (1917). Neuguinea

dysmothauma Prout, Journ. F. M. S. Mus., $17: 1$, Nordp. 51, t. 11 , f. 9 (1932) (Nordborneo). Borneo, 
cambogiodes Prout, Nov. Zool., 23, p. 24 (1916). Holl.-

Neuguinea

Rothschildi Warr., Nov. Zool., 10, p. 366 (1903). - Brit.Rothsch., Nov. Zool., 11, p. 322, t. 3, f. 21 Neuguinea (1904).

lilacina Warr., t. c., p. 365 (1903).

Brit.-

Neuguinea

iole Swinh., Tr. Ent. Soc. Lond., 1892, p. 3, t. 1, Khasiberge, f.) 7 (1892) 1894, p. 191 (1894) (Anthyria). Selangor - Hampson, Faun. Ind., Moths, 3, p. 444 (var.?) (1895).

fulviplaga Swinh., Ann. Mag. Nat. Hist., (7) 15, p. Khasiberge 165 (1905). - Hampson, Journ. Bomb. Nat. Hist. Soc., $18: 1$, p. 51 (1907).

enryodia Prout, Nov. Zool., 25, p. 80 (1918). Holl.-

Neuguinea

ignita Warr., Nov. Zool., 14, p. 139 (1907) (Brit. Holl.- u. Now Guinea).

Brit.-

Neuguinea

orgalea Meyr., Tr. Ent. Soc. Lond., 1897, p. 71 Pulo-Laut (1897).

cruoraria Warr., Nov. Zool., 4, p. 49 (1897) (Chry- Queensland, solene) (Queensland). - Turn., Proc. Linn. Brit.Soc. N. Sth. Wales, $32: 4$, p. 679 (1908); id., Neuguinea Tr. Roy. Soc. S. Austral., 46, p. 268 (1922). (var.?),

Neu-

Pommern

(var.?)

inundata Warr., Nov. Zool., 5, p. 238 (1898) (Brit. Holl.- u. New Guinea). - Turn., Proc. Linn. Soc. Brit.N. Sth. Wales, $32: 4$, p. 675 (1908). Neuguinea, cruoraria form., Turn., Tr. Roy. Soc. S. NordAustral., 46, p. 268 (1922).

auristigma Prout, Nov. Zool, 25, p. 80 (1918) (Dutch Holl.New Guinea).

Neuguinea,

Neu-

Pommern, ? VulkanInsel

phaedra Prout, t. c., p. 81 (1918).

Sudest-

Insel

charites Ch. Oberth., Et. Lép. Comp., 12, p. 165; t. Syrien 398, f. 3392 (1916) (Hyria).

subangulata Warr., Nov. Zool., 3, p. 110 (1896). Khasi-Berge

analiplaga Warr., Nov. Zool., 14, p. 138 (1907). Brit.-

Neuguinea

aurimargo Warr., Nov. Zool., 4, p. 216 (1897). - Nord-

Turn., Proc. Linn. Soc. N. Sth. Wales, $32: 4$, Queensland p. 679 (1908). - Tillyard, Ins. Austral. \& N. Z., p. 451 , t. 39 , f. 15 (1926). 
c r u o raria f. Turn., Tr. Roy. Soc. S. Austral., 46, p. 268 (1922).

lunulata Swinh., Tr. Ent. Soc. Irond., 1902, p. 661 Selangor, (1902) (N. Borneo).

Nord-

Borneo

tricolora Beth.-Baker, Ann. Mag. Nat. Hist., (8) Brit.16, p. 192 (1915)

Neuguinea

flavisparsa Prout, Nov. Zool., 23, p. 23 (1916). Holl.-

Neuguinea

elaeophragma Prout, Nov. Zool., 24, p. 314 (1917). Holl.-

Neuguinea

uncimargo Warr., Nov. Zool., 14, p. 140 (1907). Brit.-

Neuguinea

plumbeofusa Swinh., Ann. Mag. Nat. Hist., (6) 14, Khasi-Berge, p. 136 (1894) (Khasia). - Hampson, Faun. KedahInd., Moths, 3, p. 443 (1895); id., Journ. Singapore Bomb. Nat. Hist. Soc., $14: 4$, p. 652 (1903). (var.?)

altegradia Prout, Nov. Zool., 36 :2, p. 155 (1931). Luzon

semiocellata Prout, Nov. Zool, 23, p. 22 (1916) Holl.(sequ. var.?). Neuguinea

concentrica Warr., Nov. Zool., 6, p. $30 \sigma^{7}$ (1899) Brit.-

(St. Aignan). - Prout, Nov. Zool., $36: 2$, p. Neuguinea, 155 (1931). O regal is Warr., 1. c. (,O, $\mathrm{O}^{\text {r6 }}$ ex err.) (1899). Woodlark-, (St. Aignan). Bismarckund

Salomons-

Inseln

indopurpurea Prout, Nov. Zool., 23, p. 24 (1916) Holl.(Heteroctenis).

Neuguinea, ? Brit.-

Neuguinea

flavimedia Prout, t. c., p. 25 (1916) (Heteroctenis). Holl.-

ab. pers persa Prout, 1. c. (1916) (Heteroc-

Neuguinea 47

\section{Genus Pfomophyle Prout}

Prout, Nov. Zool., $37: 2$, p. 233 (1932); id. in Seitz, Macrolep., 16 , p. 52 (1933).

subcarnea Warr., Nov. Zool., 9, p. 505 (1902) Franz.(Traminda) (Congo). - Prout in Seitz, I. Guinea, 1 c., t. 6 b (1933).

Kamerun, Congo 


\section{Genus Chlorerythra Warr.}

Nov. Zool., 2, p. 91 (1895) (typus rubriplaga Warr.). - Prout in Seitz, Macrolep., 16, p. 52 (1933).

rubriplaga Warr., Nov. Zool., 2, p. 91 (1895) (S. Kapland Africa). - Prout in Seitz, 1. c., t. 6 b (1933). bis Süd-

Mossambique, ? Nyasa

var. exten uata Prout, Nov. Zool., 38:1, Kenya, p. 11 (1932) (Kenya); id. in Seitz, l. c. Brit.(1933). - rubriplaga Prout, Proc. Zool. Somaliland Soc. Lond., 1916, p. 148 (1916).

carnea Warr., Ann. S. Afr. Mus., $10: 12$, p. 495 Buschmann(1914). - - Prout in Seitz, l. c. (1933)' (huj. land 2 gen.?),

\section{Genus Traminda Warr.}

(Saalm., Lep., Madag., 2, p. 496, 1891, indescr.). - Warr., Nov. Zool., 2, p. 100 (1895) (typus atroviridata Saalm.). - Prout, Nov. Zool., 23, p. 278 (1916); id. in Seitz, Macrolep., 16, p. 52 (1933).

T' i mandra (part.) Meyr., Proc. Linn. Soc. N. Sth. Wales, (2) 2, p. 863 (1888). - (sect. 2 B) Hampson, Faun. Ind., Noths, 3, p. 460 (1895).

Gramptoloma (part.) Turn.y Proc. Linn. Soc, N. Sth. Wales, $32: 4$, p. 681 (1908).

rufistrigata Hampson, Proc. Zool. Soc. Lond., 1896, Aden, p. 267, t. 10, f. 3 (1896) (Ephyra) (Aden). Sokotra, - ? Swinh., Tr. Ent. Soc. Lond., 1904, p. Somaliland, 561 (1904) (Cosymbia). - Reb., Lep. Süd- ? Kenya arab. u. Sokotia, p. 69 (1907) (Ephyra). Prout, Proc. Zool. Soc. Lond., 1916, p. 148 (1916); id. in Seitz, Macrolep., 16, p. 53, 1. $6 \mathrm{c}$ (1933).

rufistrigula Prout in Seitz, t. c., p. 54 (1933) (er1. typogr.).

var. (?) marcida Warr., Nov. Zool., 12, p. Sudan, 27 (1905) (Cosymbia) (Sudan). - Reb. \& Kenya Zerny, Denks. Akad. Wien, 93, p. 433 (1917) (Cosymbia). - Prout in Seitz, 1. c. (1933).

acuta Warr., Nov. Zool., 4, p. 57 (1897) (Lycunges) Natal, (Natal). - Pront in Seitz, l. e. (1933). Süd-

Rhodesia

var. pallida Warr., Nov. Zool., 6, p. 296 (1899) (T.?) (Britj-Ostafrika). - Prout in Seitz, l, c., t. 6 b (1933). - ? anandaria Swinh., Tr. Ent. Soc. Lond., 1904, p. 561 (1904) (Cosymbia) (ab.?). - anandaria Hampson, Ti: Zool. Soc. Lond., $19: 2$, p. 127 (1909) (Ephyra); id., Proc. Zool. Soc. Lond., 1910, p. 476 (1910) (Ephyra). Prout, Nov. Zool., $38: 1$, p. 11 (1932); id. in Weißer Nil bis Nord-OstCongo,

NordRhodesia, ? Somaliland Seitz, l. c. (1933) (ab.?). 
falcala Warr., Nov. Zool., 4, p. 64 (1897). - Prout Natal, in Seitz, 1. c., t. $6 \mathrm{c}$ (1933).

P Portug.-

Ost-

Afrika

drepanodes Prout, Nov. Zool., 22, p. 335 (1915) $5 \mathrm{~g}(1933)$

Kamerun,

Congo,

vividaria Walk., List Lep. Ins., 23, p. 800 (1861) Senegal (Timandra) (Caffraria). - Wllgr., Ofv. Vet.Ak. Handl., 1872 : 3, p. 52 (1872) (Timandra). - Prout, Nov. Zool., 23, p. 278 (1916); id. in Ark. Zool., $18 \mathrm{~A} \mathrm{:25,} \mathrm{p.} 5$ (1926); id. in Seitz, Macrolep., 1. c., t. 6 c (1933).

Ledereri Wllgr., Wien. Ent. Monats., 7, p. 150 (1863) (Timandra) (Caffraria).

nigripunct a Warr., Nov. Zool., 4, p. 225 (1897) (Liberia). - Swinh., Tr, Ent. Soc. Irond., 1904, p. 562 (1904). - Hampson, Proc. Zool. Soc. Lond., 1910, p. 477 (1910) (Timandra).

neptunaria (part.) Swinh., 1. c. (1904) (nec Guen.).

viridaria Fawcett, Proc. Zool. Soc. Lond., 1916, p. 731 (err. pro viridaria).

ab. variegat a Swinh.| 1. c. (1904). - Prout (Macluakos) in Seitz, 1. c. (1933).

ab. $r u f a$ Prout, Nov, Zool., $37: 2$, p. 234 (1932).

(sine loc.)

atroviridaria Mab., O. R. Ent. Soc. Belg., 23, p. Unyoro, XXII (1881) (Thalera) (,Madagascar" [?err. loc.]) - Prout, Nov. Zool., 38:1, p. 11 (1932); id. in Seitz, 1. c. (1933).

o cell a ta Warr., Nov. Zool., 2, p. 100 (1895).

? Congo, Rhodesia bis Kapland

ab. $r$ u fa Warr., Nov. Zool., 4, p. 65 (1897). - (Natal) Hampson, Proc. Zool. Soc. Lond., 1910, p. 477 (1910). - Prout in Seitz, 1. c., t. $6 \mathrm{c}$ (1933).

observata Walk., List Lep. Ins., 23, p. 790 (1861). Sierra(Acidalia?) (Sierra Leone). - Prout, Nov. Leone bis Zool., 23, p. 278 (1916); id., Tr. Ent. Soc. Lond., 1927, p. 189 (1927); id. in Seitz, t. c., p. 54, t. $6 \mathrm{c}$ (1933).

gla l c a Warr., Nov. Zool., 4, p. 64 (1897) (Sierra Leone). - Hampson, Proc. Zool. Soc. Lond., 1910, p. 477 (1910) (Timandra).

striata Warr., t. c., p. 220 (1897) (Lipomelia).

\section{Angola,}

Tanganyika, Rhodesia

var. a troviridat a Saalm., Ber. Senck. Ges., Madagaskar $1879-80$, p. 293 (1880) (Timandra). decessata (nom. nov.) Saalm., Lep. Mad., 2, p. 496 , f. 262 (1891). 
syngenes Prout, Nov. Zool., 23, p. 277 (1916); Seitz, 1. c. (1933).

neptunaria Guen., Spec. Gén. Lép., 10 , p. 3, t. 18, f. 5 (1858) (Timandra) (Abyssinie), Walk., List Lep. Ins., 23, p. 799 (1861)
(Timandra). - Snell., Tijd. Ent., 15, p. 78 (1872) (Timandra). - Swinh., Tr. Ent. Soc. Lond., 1904, p. 562 (1904). - Prout, Nov. Zool., 23, p. 278 (1916); id., Proc. Zool. Soc. Lond., 1916, p. 148 (1916). - Strand, Ent. Rundsch., 35, p. 32 (1918). - Prout, Nov. Zool., $38: 1$, p. 12 (1932); id. in Seitz, l. c., t. $6 \mathrm{c}(1933)$.

ab. (?) viridipennaria Guen., 1. c. (1858) (Timandra) (Amér. sept. [err. loc.]). - Ch. Oberth., It. Isép. Comp., 20, p. 214 (1923), $22: 2$, p. 97 , t. 596 , f. 5058 (1925) (Tim.). - Prout in Seitz, 1. c. (1933).

Morphologie: Snell., l. c. (1872).

mundissima Walk., List Lep. Ins., 23, p. 795 (1861) (Acidalia) (S. Hindostan). - Cotes \& Swinh., Cat. Moths Ind., pars 4, p. 578 (1888) (Idoea). - Hampson, nl. Het., 8, p. 33 (1891) (Timandra); id., 1. c., 9, p. 40 (1893) (Gnamptoloma); id., Faun. Ind., Moths, 3, p. 460 (1895) (Timandra); id, Nat. Hist. Sokotra, p. 332 (1903) (Timandra). - Turn., Proc. Linn. Soc. N. Sth. Wales, $32: 4$, p. 682 (1908) (Gnamptoloma). - Longstaff, Butt. Hunting, p. 374 (1912). - Tams, Journ. Nat. Hist. Soc. Siam, 6:3, p. 274 (1924) (Mnesithetis). - Prout, Bull. Hill Mus., $4: 1$, p. 130 (1930).

se micompleta Walk., List Lep. Ins., 26, p. 1616 (1862) (Timandra).

dia to mata Walk., 1. c. (1862) (Timandra?) (Hindostan). - Swinh., Tr. Ent. Soc. Lond., 1884, p. 526 (1884) (Thalera).

dia to maria Swinh., Tr. Fnt: Soc. Lond, 1885 , p. 854 (1886); id., l. c., 1886, p. 454 (1886) (Timandra).

bu $u$ r m a n a Swinh., Tr. Ent. Soc. Lond., 1890, p. 214 (1890) (Timandra) (Rangoon) (ab.?). ahlorozonaria Hampson, Faun. Ind., Moths, 4, p. 563 (1896) (Gnamptoloma). Swinh., Cat. Lep. Het. Oxf. Mus., 2, p. 373 (1900) (Gnamptoloma). - Turn., T. Roy. Soe. S. Austral., 46, p. 268 (1922) (Gnamptoloma) (err. det., nec Walk.).

responsaria (part.) Swinh., Cat. Lep. Het. Oxf. Mus., 2, p. 373 (1900) (Calothysanis) (err. det.s).

ma la c o p is Lower, Tr. Roy. Soc. S. Austral,, 26, p. 228 (1902) (Timandra) (W. Australia).
Ubangi-

Schari-

Tschad

Tropisches u. Subtropisches Afrika, Natal

Solrotra

(var.?), Indien bis Hainan, Sunda-Inseln bis

Australien 
ab. submarginata Warr., Nov. Zool., 6,

p. 34 (1899) (Tambora).

var. (?) hemichroa Meyr., Tr. Ent. Soc. Brit.-

Lond., 1889, p. 488 (1889) (Timandra). Nouguinea

prasodes Meyr., Proc. Linn. Soc. N. Sth. Wales (2) Bali bis

2, p. 865 (1888) (Timandra) (Queensland). Queensland vir $i d a r i a$ Swinh., Tr. Ent. Soc. Lond., 1902, 11

p. 665 (1902) (Gnamptoloma) (Bali).

\section{Genus Gnampioloma Warr.}

Hampson, 11. Het., 9, p. 40, 1893 (indescr.). - Warr., Nov. Zool., 2, p. 95 (1895) (typus aventiaria Guen.').

Timandra sect. I[, Hampson, Faun. Ind., Moths, 3, p. 459 (1895); 4, p. 563 (1896):

aventiaria Guen., Spec. Gén. Lép., 10, p. 3, t. 18, Indion f. 5 (1858) (T.) (Australia). - Walk., List bis

Lep. Ins., 23, p. 801 (1861) (T.). - Moore, Luisiaden,

Proc. Zool. Soc. Lond., 1866, p. 365 (1866), Bismarck-

1867, p. 644 (1867) (T.); id., Lep. Ceyl., 3, Archipel

p. 458 , t. 202 , f. 4, 4a (1887) (Erastria). - (var.)

Butl., Proc. Zool. Soc. Lond., 1880, p. 687

(1880) (T.); id, Tr. Ent. Soc. Tond., 1881, p. 341 (1881) (Parasemia); id. Aun. Mag. Nat. Hist., (5) 20, p. 247 (1887) (Ochodontia). - Snell., Tijd. Ent., 24, p. 82 (1881) (T.). - A. Pagenst., Jahrb. Nass. Ver. Nat., 41, p. 173 (1888); id., ? Zoologica, $12: 29$, p. 149 (1900) (T.). - Meyr. Proc. Linn. Soc. N. Sth. Wales, (2) 2, p. 864 (1888) (T.); id., Tr. Ent. Soc. Lond., 1897, p. 73 (1897) (Calothysanis). - Tepper, Ins. S. Austrul., 2, p. 57 (1890) (T.). - Swinh., Tr. Ent. Soc. Lond., 1890 , p. 214 (1890); id., 1. c., 1894 , p. $177(1894)$ (T.); id., Cat. Lep. Het. Oxf. Mus., 2, p. 373 (1900). - Hampson. Ill. Het., 8, p. 32 (1891) (T.); 9, p. 40 (1893); id., Faun, Ind., Moths, 3, p. 459, f. 206 (1895) (T.). - Warr., Nov. Zool., 2, p. 95 (1895). - G. Semp., Reisen Philipp., (2) $6: 6$, p. 633 (1902). - Turn., Proc. Linn. Soc. N. Sth. Wales, $32: 4$, p. 682 (1908). Strand, Iris, 1910 , p. 199 (T.). - Rothsch., Iep. Br. Orn. Un. Exp., p. 95 (1915) (Gnamptolomia [sic]). - Ch. Oberth., Et. Lép. Comp., 20, p. 215, t. 549, f. 4636, 4637 (1923) (T.). - J. Joann., Ann. Soc. Ent. Fr., 98, p. 476 (1929). - Prout, Bull. Hill Mus., 4:1, p. 130 (1930); id., Journ. F. M. S. Mus., $17: 1$, p. 52 (1932).

molybdias Meyr., Tr. Ent. Soc. Lond., 1889 , p. 488 (1889) (T.) (Port Moresby) (var.?).

Morphologie: Snell., 1. c. (1881). 
B i ologie: Moore, l. c. (1887). - G. Semp., l. c., t. U, f. 12,13 (1902).

subochrea But1., Ann. Mag. Nat. Hist., (5) 20, p. Salomons245 (1887) (Ochodontia) (Alu).

? a ventiaria A. Pagenst., Zoologica, 12 : 29, Inseln, ? BismarckArchipel (var.?) 2 p. 149 (1900) (Timandra) (var.?).

\section{Genus Cosymbia Hb.}

Cyclophora (Hb., Tent.; p. [2] ined.). - Steph., Cat. Brit. Ins., 2, p. 127, 1829 (indescr.). - Moore, Lep. Ceyl., 3, p. 441 (1887) (typus pendularia Clerck). - Prout, Tr. City Lond. Ent. Soc., 10, p. 64, 65 (1901).

Cos ymbia Hb., Verz. Bek. Schnett., p. 302 (1825) (typus puppillaria Hb.). - Warr., Nov. Zool., 2, p. 92 (1895). Dyar, List N. Amer. Lop., p. 291 (1902). - Prout, Tr. City Lond. Ent. Soc., 20, p. 23 (1911); id. in Seitz, Macrolep., 4, p/ 141 (1913); id., l. c., 16, p. 54 (1933). Barnes \& Me Dunnough, Check List Lep. Bor. Amer., p. 103 (1917). - Derenne-Meyers, Faun. Belg., Géom., p. 20 (1929).

Le ucophthalmia Hb., Verz. Bek. Schmett., p. 302 (1825) (\& Leucopthalmia, err. typogr.); id., Anzeiger, p. 50 (1827) (typus ammulata Schulze). - Gmpbg." Nova Acta Acad. Leop. Nat., $49: 4$, p. 330 (1887); id., l. c., $58: 4$, p. 285 (1892) (Lencophtalmia, err. typogr.) (err. restr.). - Meyr., Tr. Ent. Soc. Lond., 1892, p. 91 (1892); Handb. Brit. Lep 27 , p. $300(1896)$ J

Cod onia Hb., Verz. Bek. Schmett., p. 302 (1825) (typus porata L.). - Rghfr. in Beck, Faun. Hernstein, $2: 2$, p. 36 (1885). - Garbsk, S. B. Akad. Wien, Math.-Nat. Cl., $101: 1$, p. 111 (1892). - Fuchs, Jahrb. Nass. Ver. Nat, 57, p. 40 (1904). - Reb. in Spuler, Schmett. Eur. (E. Hofm., Großschmett., ed. 3), 2, p. 27, f. 4 (1904); id., Berge's Schmotterlingsb. (ed. 9), p. 324 (1910). - Hoffmann \& Klos, Mitt. Nat. (Ver. Steier., 53, p. 78-82 (1917). - Hering, Tierwelt Mitteleur., Schmett.; p. 330 (1932).

Ca $\operatorname{c}$ er $a$ (part.), Tr., Schmett. Eur., 5:2, p. 437 (1825); $6: 1$, p. 343 (1827) (typus pusaria L.., Westw. sel.). Children, Philos. Mag. (n. s.) 6, p. 455 (part.) (1829). Ad. Spr., Isis, 1839, p. 124 (part.) (1839). - Hoydenreich, Syst. Verz. Eur. Sclimett., (ed. 2), p. 15 (part.) (1846): (ed. 3), p. 54 (part.) (1851).

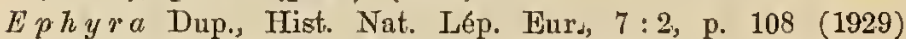
(nom. praeoce, nec Péron \& Insueur); id., Cat. Móth., p. 268 (1845). - Curt., Brit. Lep., 10, p. 447 (1833). - Bsd., Gen. et Ind. Meth., p. 220 (1840). - Brd., Mém. Soc. Emul. Doubs, 2:2, p. 137 (sep. p. 119) (1846). - H.-Sch., Syst. Bearb. Schmett. Eur., 3:24, p. 30 (1847). - Steph., List Brit. Anim. Brit. Mus., 5, 178 (1850). - Guen., Spec. 
Gén. Lép., 9, p. 405 (1858). - Staint., Man. Brit. Butt. \& Moths, 2, p. 37 (1858). - Walk., List Lep. Ins., 22, p. 624 (1861). - Peyrhff., Cat. Lép. Alsace (Bull. Soc. Colmar, 2), p. 108 (1862). - Berce, Faun. Ent. Franç., Lép., 5, p. 114 (1873). - Stdgr. \& Reb., Cat. Lep. Pal., 1, p. 276 (1901). - Barr., Lep. Brit. Isl., 7, p. 304 (1901). Eckstein, Schmett. Deutschl, 4, p. 14, t. 49, f. 6 (1923). Matella Gistl, Naturg. Thierr., p. IX (1848) (nom. nov. pro Ephyra).

Z o no s o ma Led., Verh. Zool.-Bot. Ver. Wien, 3, p. 174, 194 (sep. p. 10, 30), fig. 6 (1853). - H.-Sch., Syst. Bearb. Schmett. Eur., 6:68, p. 135 (1855); id., Samml. Außereur. Schmett., 1, p. 35 (1856). - Ad. Spr., Deutscho Schmett.kunde, p. 192 (1856). - Heinem., Schmett. Doutschl., 1, p. 708 (1859). - Stdgr.', Cat. Lep. Eur., p. 66 (1861); (ed. 2), 1, p. 152 (1871). - Constant, Cat. Lép. Saone-et-Loire, p. 205 (1866). - Suell., Vind. Ned. Macrolop., p. 568 (1867). - Bang-Haas, Nat. Tidskr., (3) 9, p. 479,480 (1874). - Ramann, Sehmett. Deutschl., 4, p. 336 (1876). - F. B. White, Scot. Nat., 3, p. 361 (1876). - Peyrliff:; Cat. Lép. Alsace (ed. 2), 1, p. 116, 117 (1880). - Rössl., Jahrb. Nass. Ver. Nat., 33/34, p. 132 (1881), - Bau, Handb. für Schmett.-Sammler, p. 236 (1886). - Poppius, Acta Soe. F. F. Fenn., 3 : 3, p. 121 (1887), - Gmpbg., Nova Acta Acad. Irop. Nat., $49: 4$, p. 329 (1887); id., l. c., $58: 4$, p. 255 (1892). - A. Fuchs, Jahrb. Nass. Ver. Nat., 46, p. 91-101 (1893). - Korb, Schmett. Mittel- Fur., p. 175, 176 (1894). - Cholodkovsky, E. Hofm., Atlas Babochek Eur., p. 243, 244, t. 58 (1897). - Bastelb., Iris, 13, p. 72, 88 (1900). - Tougemont, Cat. Lép.i Jura (Bull. Soc. Neuchatel, 29 \& 31), p. 171-173 (1203).

E u e phyra Pack., Rep. Peab. Acad. Sci., 5, p. 73 (1873); id., Mon. Geom. U. S.,.p. 365, t. 4, f. 5 (1876). - Gmpbg., Nova Acta Acad. Leop. Nat., 49:4, p. 328 (1887); $58: 4$, p. 295 (1892).

Morphologie: Breitenbach, Jena. Zeits. Naturw., 15, p. 195 (1882). - Bastelb., 1. c. (1900).

B iologie: Fritsch', Denks. Akad. Wien, Math.-Nat. Cl., $41: 1$, p. 94 (1879). - Poulton, Tr. Ent. Soc. Lond., 1884, p. 50, soq.s 57 (1884); id., Proc. Ent. Soc. Lond., 1904, p. 104, 105 (1904). - A. Fuchs, 1. c. (1893). - Tutt, Brit. Irep., 2, p. 24 (1900). - Blaschke, Raup. Eur., p. 131, 132 (1914). - Hering, Biol. Schmett., p. 97 (1926).

unocula Warr., Nov. Zool., 4, p. 217 (1897) (Dar- Tanganjika, es-Salaam). - Prout in Seitz, Macrolep., 16, Nyasa, p. 54 , t. 6 e (1933).

? Transvaal

pendularia Clerck, Ic. Ins. Rar., 1, t. 7, f. 5 (1759) Nord- u. [Suecia]. - I., Faun. Suec. (od. 2), p. 327 Zentral(1761). - [Schiff.] Schmett. Wien, p. 107 Europa, (1775). - Gözc, Degeer Abh. Gesch. Ins., Italien, $2: 1$, p. 263 (1778); id., Ent. Beytr., $3: 3$, Sibirien p. 361 (1781). - Rossi, Faun. Etrusca, 2, p. 192 (1790); (ed. ㅍll), 2, p. 308 (1808). Bkh., Naturg., Eur. Schmett., 5, p. 255 (1794). - Hb., Samml. Eur. Schmett., Geom., 
t. 13, f. 66 (1798); Tent., p. [2] (ined.) (Cy.); Verz. Bek. Schmett., p. 303 (1825) (Cod.). - Ill., Schmott. Wien (n. od.), p. 424 (1801). - Schrank, Faun. Boica, 2 :2, p. 25 (1802). - Scharfenberg in Bchst. \& Scharfenb., Schädl. Forstins., 3, p. 689 (1805). - Haw., Lop. Brit., 2, p. 311 (1809) (Geometra). - Tr., Schmett. Eur., 6:1, p. 361 (1827) (Cab.). - Freyer, Beitr. Gesch. Eur. Schmett., 2, p. 19, t. 54, f. 2 (1828) (Cab.); id., Isis, 1839 , p. 732 (1839) (Geometra). - Dup., Hist. Nat. Lép. Eur., $7: 2$, p. 108 $(1829) ; 8: 1$, p. 35, t. 172, f. 5 (1830) (E.). - Steph., Cat. Brit. Ins., 2, p. 127 (1829) (Oy.); id., Ill. Haust., 3, p. 199 (1831) (E.). - Wood, Index Ent., 87, t. 20, f. 528 (1835) (Phalaena). - Zoll., Isis, 1839, p. 299 (1839) (Geometra). - H.-Sch. in Panz., Deutschl. Ins., 172, p. 103 (1840) (Idaea). - Westw. in Humphr. \& Westw., Brit. Moths, 2, p. 25, t. 61 , f. $18,19,24,25$ (1843?) (E.). - Eversm., Faun. Lep. Volg.Ural., p. 396 (1844) (Cab.). - H.-Sch., Syst. Bearb. Schmett. Eur., 3 : 24, p. 31 (1847) (E.); $3: 31$, t. 58 , f. 357 (1848); $6: 55$, p. 70 (1852) (E. sp., s. n.); $6: 60$, t. 88 , f. 540 (1853) (pendularia [ab.]). - Led., Verh. Zool.-Bot. Vor. Wien, 3, p. 174 (sep. p. 10) (1853) (Z.). - Fologne, Ann. Soc. Ent. Belg., 3, p. 126 (1857) (E.). - Guen., Spec. Gén. Lép, 9, p. 413 (1858) (E.). - Kltb., Phytophagen (Verh. Pr. Rheinl. 15), p. 124 (1858) (Cab.). - Heinem., Schmett. Deutschl., 1, p. 711 (1859) (Z.). - Zebrawski, Motyl. Krakowa, p. 185 (1860) (Cab.) - Walk, List Lep. Ins., 22, p. 630 (1861) (E.). - Snell., Vlind. Ned. Macrolep., p. 570 (1867) (Z.). - Gavere, Tijd. Fint., 10, p. 212 (1867) (Z.). - Nolcken, Iep. Faun. Estl. (Arb. Nat. Ver. Riga), 1, p. 232 (1S68) (Z.). - Newm., Brit. Moths, p. 74 (1869) (E.). - Stdgr., Cat. Lep. Eur. (ed. 2), 1, p. 152 (1871) (Z.); (ed. 3) 1, p. 276 (1901) (E.). - Ramann, Schmett. Deutschl. 4, p. 336 , t. 59, f. 39 (1876) (Z.). - Frey, Lep. Schweiz, p. 196 (1880) (Z.). - Poppius, Acta Soc. F. F. Fenn., $3: 3$, p. 123 (1887) (Z.). - W. Potersen, Lep.-Faun. Arkt. Eur., in Beitr. Kenntn. Russ. Reiches, (3) 4, p. 33, 111 (1887) (Z.). - Ch. Auriv., Nord. Fjäril., p. 206 (1891) (Z.). - Meyr., Tr. Fint. Soc. Lond., 1892, p. 91 (1892) (L.); id., Handb. Brit. Lep., p. 245 (1895); id., Rev. Handb, p. 207 (1927) (L.). - Gmpbg., Nova Acta Acad. Leop. Nat, $58: 4$, p. 289 (1892) (Z.). - A. Fuchs, Jahrb. Nass. Vier. Nat., 46, p. 100 (1893) (Z.). - Kane, Entom., 30, p. 129 (1897); id., Lep. Ire- 
land, p. 102 (1901). - Herz, Iris, 11:2, p. 260 (1899) (Z.). - Strand, Nyt Mag. Naturvid., 39, p. 30 (1901) (Z.). - Barr.y Iep. Brit. Isl, 7 , p. 324 , t. 328, f. $2-2 \theta$ (1901) (E.). - Woodforde, Entom., 35, p. 275 (1902) (Z.). - A. Pagenst. in Roemer \& Schaudinn, Faun. Aret, 2:2, p. 306 (1902) (Z.). - Reb. in Spuler, Schmett. Eur. (E. Hofm., Großschmett., ed. 3) 2, p. 27 , t. 40 , f. 11 , t. 58 , f. 18 (1904) (Cod.); id., Berge's Schmetterlingsb. (ed. 9), p. 324, t. 40, f. 6 (1910) (Cod.). - Riedel, Iris, 18, p. 151 (1905) (E.). - South, Moths Br. Isl., 2, p. 139, t. 53, f. 1, 2 (1908) (E.). - Vorbrodt \& Müll.-Rutz, Lep. Schweiz, 2 : 1, p. 27 (1913) (Cod.). - Prout in Seitz, Macrolep., 4, p. 142, t. $4 \mathrm{n}$ (1913). - Klöcker, Danm. Fauna, 17, p. 21, t. 2, f. 35 (1915) (E.). - Bowman, Proc. S. Lond. Ent. Soc., $1916 / 17$, p. 116 (1917), 1917/18, p. $66(1918), 1920 / 21$, p. 85 (1921) (Z.). - Culot, Noct. \& Géom. Eur., 3, p. 86, t. 13, f. 255 (1918) (E.). - Stephan, Iris, 39 , p. 71 (1925) (Cod.). - Derenne-Meyers, Faun. Belg., Géom., p. 20 (1929). - Adkin, Moths Eastbourne, 1, p. 65, t. 24, f. 1-3 (1930) (E.). - Vorbrodt, Mitt. Schweiz. Ent. Ges., $14: 6$, p. 326 (1930) (Cod.). Hering, Tierwelt Mitteleur., Schmett., p. 330, f. 544 (1932) (Cod.).

albipunctata Hufn., Berl. Mag,, 4, p. 526 (1767). - Gleditsch, Forstwissensch., 1, p. 521 (1775). - Ratt., Naturf., 11, p. 74 (1777). - Scharfenberg in Scriba's Journ., 3 , p. 231, 239 (1791).

s us pens a Retz., Gen. et Spec. Ins., p. 43 (1783).

pupillaria Brahm, Ins.-Kal., 2:1, p. 385 (1791) (Mainz).

pandularia F., Supp. Ent. Syst., p. 451 (1798).

circularia F., l. c. (1798) (nom. prateoce.): a mataria. Vallot, Concord. Réaumur, p. 26 (1802) (nec Linn.).

v us armana Walk., List Lep. Ins., p. 637 (1861) (E.) („Ceylon" [err. loe.]).

pe ndularium Wllgr., Ind. Noct. \& Geom. Scand. (Bih. Sv. Vet.-Ak. Handl,, 2:4), p. 22 (1874) (Z.). - Reutti, Irep. Baden (ed. Meess \& Spuler), p. 115 (1898) (Z.).

ab. decoraria Newm., Zool., 19, p. 7798 (1861) (E.?) (S. England); 20, p. 7874 (1862). - Prout in Seitz, Macrolep., 4, p. 142 (part.) (1912); id., Entom., 53, p. 52 (1920). - L. W. Newm., Proc. Sth. Lond. Ent. Soc., 1916/17, p. 113 (1917). - Woodforde, Entom., 54, p. 286 (1921). - Möbius, Iris, 36, p. 77 (1922). - pendularia var. Miller, Zool., 19, p. 7803 (1861); id., 
Proc. Ent. Soc. Lond., (3) 1, p. 23 (1862). - Bond, Entom., 9, p. 217, fig. (1876). Barr., Lep. Brit. Isl., 7, p. 325 , t. 328 , f. $2 \mathrm{~d}$ (1901). - subroseata Bowman, Ent. Rec., 27, p. 141 (1915). - Schawerda, Zeits. Oesterr. Ent.-Ver.: 10, p. 47 (1925) (nec Woodforde). - nigroroseate H. WV. Wood, Entom., 49, p. 80 (1916). - nigrosubroseata Bowman, t. c., p. 117 (1917); 1917/18, p. 66 (1918); $1920 / 21$, p. 85 (1921).

ab. ra dia ta Delahaye, Mém. Soc. Angers, (5) 2, p. $180(1900)$ - Prout in Seitz, Macrolep., 4, p. 142 (1913) (Pignerolles).

ab. (var.?) subrose at a Woodforde, Entom., 35, p. 276 (1902). - South, Moths Brit. Isl., 2 , p. 139 , t. 53 , f. 3 (1908). - G. W. Mason, Entom., 44, p. 409 (1911). - Prout, Entom., 53, p. 54 (1920). - Bowman, Proe. Sth. Lond. Fint. Soc., 1920/21, p. 85 (1921). - ? Sterneck, Prodr. Sclimett. Böhn., p. 160 (1929). - decoraria (part.) Prout in Seitz, Macrolep., 4, p. 142, t. 5 c (1913).

ab. ianthinarium Stichel, Berl. Ent. Zeits., 1901, SB., p. 20 (1901) (pr. sub-ab.) - janthinaria Reb., Berge's Schmetterlingsb., (ed. 9), p. 324 (1910). - Prout, Entom., 53, p. 54 (1920):

ab. ob sole taria Lambillion, Cat. Lép. Belg., (16), p. 248 (1905). - Prout in Seitz, Macrolep., 4, p. 143 (1913). - ? Osthelder, Schmett. Südbayerns, 1:3 (Mitt. Münchn. Eint. Ges., 19, Beilage), p. 394 (1929).

ab. Linearia Lambillion, 1. c. (1905). Prout, t. c., p. 143 (1913).

ab. brunnearia Lambillion, 1. c. (1905). Prout, l. c. (1913).

ab. nigrostriata Lutzau, Korr.-Bl. Nat. Ver. Riga, 51, p. 30 (1908). - Prout, l. c. (Livland), (1913).

ab. de pu $l s$ a Bastelb., Iris, 20, p. 263 (1908).

- Prout, 1. c. (1913). - Vorbrodt \& Müll.Rutz, Schmett. Schweiz, $2: 6 / 8$,p. 632 (1914). - Nolcken, Arb. Nat. Ver. Riga, 2, p. 232 (1868) (s. n..).

ab. subochreata Woodforde, Ent. Mo. Mag., 46, p. 115 (1910). - Prout, t. c., p. 142 (1913).

ab. flavescens Prout, t. c., p. 143 (1913). - Schwarz, Kleem. Beytr., $2: 1$, p. 17, t. 3, f. 1,2 (1793) (s. n.).

ab. impictaria Meves, Ent. Tidskx., 35, p. 124 (1914).

ab. orbiculoides Woodforde, Ent. Mo. Mag., 55, p. 103 (1919).

ab. hatertica V. G. M. Schultz, Int. Ent. Zeits., $25: 18$, p. 180, t. 2, f. 35 (1931).

f. (gen. aest.) a estiva Vorbrodt, Mitt. Schweiz. Ent. Ges., $14: 6$, p. $326(1930)$. (Tessiu) 
var. griseolata Stdgr., Iris, 10, p. 23 Amur, (1897); id., Cat. Irep. Pal., 1, p. 276 (1901). - ? W. Petersen, Lep. Estl., p. 116 (1902); (ed. 2) 1, p. 229 (1924). - ? Ter Haar, Onze Vlind., p. 260 (1904) (ab.). - ? Slevogt, Arb. Nat. Ver. Riga (n. s.) 12, p. 171 (1910). - - ? Prout in Seitz, Macrolep., 4, p. 142 (1913) (ab.). - ? Culot, Noct. et Geom. Eur., 3, p. 87, t. 13, f. 256 (1918).

Morphologie: H.-Sch., System. Bearb. Schmett. Iur., 3:8, Umrißtaf., p. VIII, XXXIV, t. 8, f. 33 (1844). - Loed., 1. c.s f. 6 (1853). - Bastelb., Iris, 13, p. 80, t. 2, f. 2 (1900). - W. Petersen, Mém. Acad. Sci. St. Pét., (8) 9, p. $95(1900)$. - Peyron, K. Vet. Akad. Handl., 44:1, p. 206, t. 8, f. 19 (1909) (ovum). - E. Petersen; Jena. Zeits. f. Nat., 47, p. 182 (1911). Pieroe, Genit. Geom. Brit., p. 37, t. 23 (1913).

B i ologi e: Clerck, I. c. (1759). - Degeer, Mém. Hist. Ins., 2, p. 300, t. 6, f. 7 (1771). - Göze, l. c. (1778). - Hb., Gesch. Eur. Schmett., Larv., 5, Geom., 1, Ampliss. L. a, b, f. 1, a-c. - Freyer, 1. c. (1828). Trap in Sepp, Besch. Wond. Gods, 6, p. 107, t. 26 (1842?). - Gartner, Geom. u. Microlep. Brünn (Verh. Naturf. Ver. Brünn, 4), p. 23 (1866). - Nolcken, l. c. (1868). - Porritt, Ent. Mo. Mág., 10, p. 71 (1873). - Cole, The Ent., 8, p. 125 (1873). Praun, Abbild. Eur. Schmett.-Raupen, t. 2, f. 7 (1875). - Ramann, l. c., t. 1, f. 30 (1876). - O. Wilson, Larv. Brit. Lep., p. 103, t. 20, f. 8 (1880). - Poulton, Tr. Ent. Soc. Lond., 1884, p. 38 seq., t. 1 , f. 11 , 12 (1884); id., Philos. Tr. Roy. Soc., 178 B, p. 437 (1887). - Redclyffe, The Tint., 17, p. 280 (1884). - E. Hofm., Raupen Gr.-Schm. Eur., p. 175, t. 40, f. 11 (1893). - Buckler, Larv. Brit. Butt. \& Moths, 7, p. 66, t. 115, f. 10 (1897). - Barr., 1. c. (1901). Woodbridge, Entom., 34, p. 179 (1901). Uffeln, Großschmett. Westfal., p. 111 (1908). - Dadd, Int. Ent. Zeits., 5, p. 222 (1911); id., Berl. Ent. Zeits., S.B., p. 29 (1912). Aue, Ent. Anz., 10, p. 207 (1930).

Hy br. pendulo-orbicula (pendularia $0^{\circ} \times$ orbicularia of) Tutt, Brit. Lep., 5, p. 541 (1907).

Hybr. s. n. (pendularia $\mathrm{O}^{n} \times$ annulata क) L. W. Newm., Proc. Ent. Soc. Iond., 1912, p. 102 (1912); id., Entom., 46, p. 21 (1913) (E.) (ab.).

?Finnland, PBaltische

Länder 
pendulinaria Guen., Spec, Gén. Lép., 9, p. 414 Nond(1858) (E.). - Walk., List Lep. Ins., 22, Amerika p. 631 (1861) (E.). - Pack., Mon. Greom. U. S., p. 363 , t. 10 , f. 72 (1876). - Möschl., Stett. Fint. Zoit., 47, p. 175 (1886) (Acidalia). - Gmpbg., Nova Acta Acad. Leop. Nat., $58: 4$, p. 292 (1892) (Z.). - Ch. Oberth., Et. Lép. Comp., 12, p. 156, t. 395, f. 3354 (1916) (E.). - Blackmore, Can. Ent., 53, p. 168 (1921).

$q u a d r a n n u l$ at $a$ Walk., List Lep. Ins., 26, p. 1595 (1862) (Acidalia).

lumenaria Hulst, Ent. News, 6, p. 106

(1895) (Arrhostia) (err. det., nee Hb.-Gey.); id., Tr. Amer. Ent. Soc., 23, p. 300 (1896) (L.). - Dyar, List N. Amer. Lep., p. 292 (1902). - Barnes \& Mc Dunnough, Check List Lep. Bor. Amer., p. 103 (1917). Strand, Archiv f. Naturgi, 1922, A. 8, p. 271. - Forbes, Lep. New York, p. 13 (1923); in Leonard, etc., Ins. New York, p. $392(1928)$. var. (?) $g r i s e$ or Me Duunough, Can. Ent.,
59, p. 240 (1927). - lumenaria Dyar, Proc. U. S. Mus., 27, p. 901 (err. det., nec Hb.Gey.) (1904).

B i o logie: Pack., l. c., t. 13, f. 14-14 b (1876). - Dimmock, Psyche, $4: 132 / 134$, p. 176 (1885). - Scudd $v$ Psyche, 8, p. 103 (1897). - Dyar, 1. c. (1904); id,, Psyche, 13, p. 93 (1906): - Forbes, l. c. (1923); 1. c. (1928).

orbicularia Hb., Samml. Eur. Schmett., Geom., t. Zentral12, f. 60 (1798) (Europa); id, Verz. Bek. Europa, Schmett., p. 302 (1825). - Haw., Lep. NordBrit., 2, p. 111 (1809) (Geometra). - Tr., Spanien. Schmett. Eur., 5 : 2, p. 438 (1825), $6: 1$, p. bis Süd364 (1827) (Cab.). - Steph., Cat. Brit. Ins., Rußland 2, p. 127 (1829) (Cy.); id., Ill. Haust., 3 , p. 199,328 (1831) (E.). - Wood, Index Ent., 87, t. 20 , f. 529 (1835) (Phalaena). - H.-Sch. in Panzer, Deutschl. Ins., 172, p. 103 (1840) (Idaca); id., Syst. Bearb. Schmett. Eur., $3: 24$, p. 31 (1847) (E.). - Westw. in Humphr. \& Westw., Brit. Woths, 2, p. 25, t. 61 , f. 20 (1843?) (E.). - Eversm., Faun. Lep. Volg.-Ural., p. 396 (1844) (Cab.). - Led., Verh. Zool-Bot. Ver. Wien, 3, p. 174 (sep. p. 10) (1853) (Z.) - Guen., Spec. Gén. Lép., 9, p. $413(1858)$ (E.). Jenner, Zool., 16, p. 6103 (1858) (Ephyra). - Heinem., Schmett. Deutschl., 1, p. 710 (1859) (Z.). - A. B., Ent. Weekl. Int., 10, p. 48 (1861). - Snell., Vlind. Ned. Macrolep., p. 570 (Z.). - Nowm., Brit. Moths, p. $73(1869)(E$.$) - Heyl. in Sepp, Ned.$ Ins., (2) 3, p. 69 , t. 13 , f. $1-10$ (1872)

Brit.-

Columbia 
(2.). - Berce, Faun. Ent. Franç., Lép., 5, p. 120 (1873) (E.). - Frey, Lep. Schweiz, p. 73 (1869) (E.). - Heyl. in Sepp, Ned. Microlep., p. 1171 (1882) (Z.). Kolbe, Berl. Ent. Zeits., 27, p. 223 (1883) (Cab.). - Poppius, Acta Soc. F. F. Fenn., $3: 3$, p. 122 (1887) (Z.). - Ch. Auriv., Nond. Fjär.s p. 206 (1891) (Z.). - Gmpbg., Nova Act. Acad. Leop. Nat., $58: 4$, p. 290 (1892) (Z.). - Meyr., Tr. Ent. Soc. Lond., 1892, p. 91 (1892); id., Handb. Br. Lop., p. 244 (1895); id., Rev, Handb., p. 207 (1927) (L.). W. F. Kirby, Handb. Lep., 5, p. 223 (1897) (Cy.). - Barr., Lep. Brit. Isl., 7, p. 320, t. 328 , f. 1, 1a (1901) (E.). - Stdgr. \& Reb. Cat. Lep. Pal., 1, p. 276 (1901) (E.). Reb. in Spuler, Schmett. Eur. (E. Hofm., Großschmett., ed. 3), 2, p. 27, t. 58, f. 19 (1904) (Cod.); id., Berge's Schmetterlingsb., (ed. 9), p. 324 (1910) (Cod.). - South, Moths Brit. Isl., 2, p. 139, t. 53, f. 4, 5 (1908) (E.). - Uffeln, Großschmett. Westfalens, p. 111 (1908) (E.). - Ribbe, Lep.Faun. Andal. (Iris, 23, Beih. 3/4), p. 311 (1912) (Cod.). - Vorbrodt \& Müll.-Rutz, Schmett. Schweiz, $2: 1$, p. 27 (1913) (Cod.). - Prout in Seitz, Macrolep., 4, p. 143, $i$. 4 n (1913). - Culot, Noct. \& Géom. Eur., 3, p. 87 , t. 13 , f. 257 (1918) (E.). - DerenneMeyers, Faun. Belg., Géorn., p. 21 (1929).

orbicularium Wllgr., Index Noct. et Geom. Scand. (Bih. Vet.-Ak. Handl., $2: 4$ ), p. 22 (1874) Z.). - Reutti, Lep. Baden (ed. Meess \& Spuler), p. 116 (1898) (Z.).

ab. namurcensis (Snell., Tijd. Ent., 38, p. 53 , t. 4 , f. 4 , 1895 , в. n.) Lambillion, Ann. Soc. Ent. Namur, 5, p. 249, t. 2 (Cat. Lép. Belg. fasc. 16) (1905). - Prout, 1. c. (1913). - Derenne-Meyers, 1. c. (1929). ianthinarium Prout, Ent. Rec., 24, p. 25 (1912) (err. det., nec Stichel).

(Namur)

Morphologie: E. Brandt, Hor. Soc. Ent. Ross., 15, p. 79 (1879) (Cidaria). - W. Petersen, Móm. Acad. Sci. St. Pét., (9) 6, p. 11, schema O (1900). - Bastelb., Iris, 13, p. 80 , t. 2, f. 1 (1900). - Pierce, Genit. Geom. Brit., p. 37, t. 23 (1913).

B i ol o g i e: Wilde, Pflanz. u. Raup. Deutschl., 2, p. 374 (1861). - Burney, Ent. Weekly Int., 9, p. 166 (1861). - Greene, Zool., 19, p. 7760 (1861). - Heyl., l. c. (1872?). Porritt, 10, p. 97 (1877). - O. Wilson, Larv. Brit. Lep., p. 102, t. 20, f. 7 (1880). - Poulton, Tr. Fint. Soc. Lond., 1884, p. 38 seq. (1884); id., Philos. Tr. Roy. Soc., 178B, p. 437 (1887). - S. Walker, Ent. Rec., 4, p. 269 (1893). - E. Hofm., Raup. d. Groß- 
schmett. Eur., p. 175 (1893); Supp., tab. 6, 1. 12 (1910). - Buckler, Larv. Brit. Lep., 7, p, 65, t. 115, f. 9 (1897). - Barr. l. c. (1901). - South, 1. c., t. 51, f. 1-1b (1908). - Chapman, Tr. Ent. Soc. Lond., 1916, p. 305, t. 80, f. 2 (1916). - Harrison; Proc. Ent. SSoc. Lond., 3, p. 18 (1928).

Hybr. brightoni (orbicularia $\sigma^{7} \times$ linearia O) Tutt, Ent. Rec., 17, p. 284 (1905) (indescr.); id., Brit. Lep., 5, p. 29, 38 (1907). ? Vorbrodt \& Müll.-Rutz, Lep. Schweiz, $2: 1$, p. 28 (1913). - Staint., Ent. Weekl. Int., 8, p. 47 (1860) (s. n.); id., Ent. Ann. 1861, p. 100 (1860) (s. n.). - Cooke, Proc. Ent. Soc. Lond., (2) $5: 8$, p. 116 (1861) (s. n.).

H y b $x$. orbiculo-pendula (orbicularia on $\times$ pendularia Q), Tutt, Brit. Leps, 5, p. 541 (1907). - Prout in Seitz, Macrolep.' 4, p. 143 (1913).

H y b r. Headi (orbicularia or $\times$ annulata \&) Tutt, t. c., p. 542 (1907). - Prout, t. c., p. 144 (1913).

albiocellaria $\mathrm{Hb}$, Beitr. Gesch. Schmett., $1: 4$, p. Zentral3 , t. 1 A (1789) (Wien); 2 addenda, p. u. Süd118 ( 1793$)$. - Bkh., Naturg. Eur. Schmett., Europa, 5, p. 265 (1794). - Esp., Schmett. in Abbild., Kleinasien, $5: 9$, p. 240 , t. 43 , f. 7 (1803). - H.-Sch., Transcaspien Nene Schmett. Eur., 1, p. 4, f. 32 (1856) (Z.). - Guen., Spcc. Gén. Lép., 9, p. 412 (var.?) (1858) ( $E$, albiocellara). - Heinem., Schmett. Deutschl., 1, p. 710 (1859) (Z.). - Walk., List Lep. Ins., 22, p. 628 (1861) (E.). Stdgr., Cat. Lep. Eur., p. 66 (1861) (Z.); (ed. 2), 1, p. 153 (1871) (Z.); (ed. 3), 1, p. 276 (E.). - Bienert, Lep. Ergebn. Reis. Pers., p. 38 (1870) (Z.). - Sand, Cat. Lép. Berry \& Auv., p. 89 (1879) (Z.). Christoph in Romff., Mém. Lép., 3, p. 94 (1887); 5, p. 48 (1889) (Z.). - Gmpbg., Nova Acta Acad. Leop. Nat., $58: 4$, p. 287 (1892) (Z.). - Garbsk., SB. Akad. Wien, Math.-Nat. Cl., $101: 1$, p. 111 (1892) (Cod.). - Meyr., Tr. Ent. Soc. Lond., 1892, p. 91 (1892). - Bastelb., Jahrb. Nass. Ver, Nat., 53, D. 201 (1900) (Z.); id., Int. Ent. Zeits.. 20, t. 2, f. 4 (1906), - Reb., Ann. Hofm. Wien, 19 , p. 254 (1904) (Cod.); id. in Spuler's Schmett. Eur. (E. Hofm. Großschmett. ed. 3) 2, p. 27, t. 58, f. 21 (1904) (Cod.); id., Berge's Schmetterlingsb. (ed.9) p. 324 (1910) (Cod.). - Ribbe, Lep.-Faun. Andal. (Iris 23, Beih. 3/4), p. 311 (1912) (Cod.). - Vorbrodt \& Müll.-Rutz, Schmett. Schweiz, $2: 1$, p. 28 (1913), 6/8, p. 639 (1914) (Cod.). - Prout in Seitz, Macrolep., 4, p. 144, t. $4 \mathrm{n}$ (1913). - Ch. Oberth., Et. Lép. Comp., 12, p. 155 (1916). - Culot, 
Noct. \& Géom. Eur., 3, p. 87, t. 258 (1918)

(E.). - Zerny, Eos, 3, p. 406 (1927). Warnecke, Amat. Pap., 6, p. 86 (1932); id., Ent. Zeits. (Frankf.), 47, p. 53, 64, f. 1-6 (1933) (E.). - Lhomme, Amat. Pap., 6, p. 92 (1932). - Hering, Tierwelt Mitteleur., Schmett., p. 331, p. 545 (1932) (Cod.).

a c e llaria Hb., Hb., Samml. Eur. Schmet.t., Geom., t. 13, f. 64 (1798); id., Verz. Bek. Schmett., p. 302 (1825) (L.). - Tr., Schmett Eur., 5:2, p. 438 (1825) (Cab.); 6:1, p. 360 (1827) (Cab.). - Dup., Hist. Nat. Lép. Eur., $8: 1$, p. 33, t. 172, f. 4 (1830) (E.). - Steph., Ill. Haust., 3, p. 200 (1831) (E.); id., List Brit. Anim. Brit. Mus., 5, p. 299 (1850) (E.). - Wood, Index Ent., p. 251, t. 54, f. 60 (1839) (Phalaena): - H.-Sch. in Panzer, Deutschl. Ins., 172, t. 102 (1840) (Idaea). - Eversm., Taun. Lep. Volg.-Ural., p. 396 (1844) (Cab.). - Led., Verh. Zool.-Bot. Ver. Wien, 3, p. 174 (sep. p. 10) (1853) (Z.).

argusaria Bsd., Gen. et Ind. Meth., p. 220 (1840) (E.). - H.-Sch., Syst. Bearb. Schmett. Eur., $3: 8$, p. 3 (1844) (s. gen.); $3: 24$, p. 30 (184T) (E.). - Dup., Cat. Méth. Lép. Eur., p. 269 (1845) (E.).

gen. aest. the $v$ in $a$ t $a$ Bastelb., Tahrb. Nass. Ver. Nat., 53, p. 208 (1900). - Naufock, Jahresb. Wien. Ent. Ver., 12, p. 72 (1902). - Reb., Ann. Hofmus. Wien, 25, p. 354 (1911) (therinaria). - Culot, 1. c., f. 259 (1918). - Warmecke, 1. c. (1932). - thermata Bastelb., 1. c., f. 5 (1906).

Morphologie: Bastelb., Iris, 13, p. 81, t. 2 , f. 3 (1900).

B i ologie: Streckfuß, Berl. Ent. Zeits., 33 : 2 , p. 309 (1890). - H. May senr., Jahresb. Wien. Ent. Ver., 2, p. 27 (1892) - I. Hofm., Raup. Großschmett. Eur., p. 175 (1893); supp.-tab., 6, f. 13 (1910). - Bastelb., 1. c. $(1900)$.

Iennigiaria A. Fuchs, Stett. Fnt. Zeit., 41, p. 268 (1883) (Z.). - Gmpbg., Nova Acta Acad. Leop. Nat., $58: 4$, p. 290 (1892) (Z.). H. May senr., Jahresb. Wien. Ent. Ver., 2, p. 29 (1892) (Z.). - Bastelb., Iris, 13, p. 81 (1900); id., Jahrb. Nass. Ver. Nat., 53, p. 201 (1900) (Z. lenigiaria). - Stdgr. \& Reb., Cat. Lep. Pal., 1, p. 277 (E. albiocellaria var.). - Reb. in Spuler, Schmett. Eur., 2, p. 27, t. 71 a, f. 24 c (1903); id., Berge's Schmetter]ingsb. (ed. 9), p. 324 (1910) (Cod. albiocellaria var.). - Prout in 
Seitz, Macrolep., 4, p. 144, t. 4 n (1913). Heinrich, Macrolep. Digne, p. 99 (1923); id., Deutsch. Ent. Zeits., 1928, p. 202 (1928) (Cod.). - Warnecke, Amat. Pap., 6, p. 86 (1932). - Lhomme, ibid., p. 92 (1932). Hering, Tierwelt Mitteleur., Schmett., p. 331, f. 546 (1932) (Cod.). - Warnecke, Ent. Zeits. (Frankf:), 47, p. 53, 64, f. 7-12 (1933) (E.).

a lbi o cellaria A. Fuchs, Stett. Ent. Zeit., 41 , p. 94 (1880) (Z.). - Reichenau, Jahrb. Nass. Ver. Nat., 58, p. 275 (1905) (E.) (err. det.).

gen. aest. a e 8 tiva A. Fuchs, ibid., 44, p. 270 (1883); id., Jahrb. Nass. Ver. Nat, 46, p. 98 (1893). - Prout, 1. c. (1913).

var. (?) occidentalis D. Luc., Bull. Soc. Frankreich Ent. Fr., 37, p. 166 (1932).

Morphologie: Bastelb., Iris, 13, p. 81 (1900).

Biologie: A. Fuchs, Stett. Ent. Zeit., 44, p. 271 (1883). - E. Hofm., Raup. Großschmett. Eur., p. 176 (1893). - Bastelb., Jahrb. Nass. Ver. Nat., 53, p. 201 (1900).

annulata Schulze, Naturf., 6, p. 92, t. 4 (1775). - England u. Göze, Ent. Beytr., $3: 3$, p. 388 (1781). - Frankreich Guér. in Tabl. Encycl. et Méth., 18, Ins., p. 25, t. 89 , f. 3 (1818). - Wernb., Beitr. Schmettkunde., 1, p. 307 (1864) (Geometra). - Snell., Tijd. Ent., 8, p. 55 (1865) (Z.); id., Vlind. Ned. Macrolep., p. 570 (1867) (Z.). - Stdgr., Cat. Lep. Eur., (ed. 2) 1, p. 153 (1871) (Z.); (어. 3) 1, p. 277 (1901) (E.). - Borgmann, Anleit. Schmett. Cassels, p. 150 (1878) (Z.). - F. Schmidt, Arch. Fr. Nat. Mecklenb., 33, p. 167 (1880) (Z. amulata [err. typogr.(]). - Lampa, Ent. Tidskr., 6, p. 91 (1885) (Z.). - Gmpbg., Nova Acta Acad. Leop. Nat., $54: 5$, p. 522 (1890) (E.). - Ch. Auriv., Nord. Fjär., p. 206 (1891) (Z.). - Meyr., Tr. Ent. Soc. Lond., 1892, p. 91 (1892); id., Handb. Brit. Lep., p. 246 (1895); id., Rev. Handb., p. 208 (1927) (t..) - Hormuzaki, Unters. Lep.-Faun. Bukovina, p. 69 (1894) (Z.); id., Verh. Zool.-Bot. Ges. Wien, 49, p. 38 (1899) (Z. annullata). - Caradja, Iris, 9, p. 68 (1897) (Z.). - Tuit, Proc. Ent. Soc. Iond., 1898, p. 23 (1898) (Z.). - Schröder, Anch. Fr. Nat. Mecklenb., $57: 2$, p. 159 (1903) (E.). - Reb. in Spuler, Schmett. Eur. (E. Hofm., Großschmett., ed. 3), 2, p. 27 , t. 58 , If 20 (1904) (Cod.); id., Berge's Schmetterlingsb., (ed. 9) p. 325, tt. 40, f. 7 (1910) (Cod.). - South, Moths Brit. Isi., 2, p. 138, t. '55, 4. 6, 9 (1908) (E.). - Vorbr. \& Müll.-Rutz, Schmett. Schweiz, $2: 1, \mathrm{p}$. 
28 (1913) (Cod.). - Prout in Seitz, Macrolep., 4, p. 145 , t. 4 o (1913). - Klöcker, Danm. Fauna, 17, p. 21, t. 2, f. 36 (1915) (E.). - Culot, Noct. \& Géom. Eur., 3, p. 88, t. 13, f. 261 (1918) (E.).

a n $n$ u laria F., Syst. Ent, p. 625 (1775); id., Spec. Ins., 2, p. 249 (1781); id., Mant. Ins., 2, p. 190 (1787); id., Fnt. Syst., 3:2, p. 147 (1794). — De Villers, Linn. Ent., 2, p. 318 (1789). - Gm., Linn. Syst. Nat., $1: 5$, p. 2467 (1790). - Bork. in Scriba, Beitr. Ins.-Gesch., 1, p. 28, t. 3, f. 3 (1790). - Scharfenberg in Scriba, Journ, 3, p. 230 (1791). - Seriba, ibid., p. 243 (1791). - Wernb., Beitr. Schmettkunde., 2, p. 331 (1864) (Geometra).

o micronaria [Scbiff.] Schmett. Wien, p. 107 (1775); (ed. Пl.) p. 425 (1801). - Hb., Beitr. Gesch. Schmett., $1: 4$, p. 25, t. $4 \mathrm{U}$ (1789); id., Samml. Eur. Schmett., Geom., t. 13, f. 65 (1798); id., Iep. Zutr., p. 12 (1820) ( $\left(y_{y}\right)$; id., Verz. Bek. Schmett., p. 302 (1825) (L.). - Fibig \& Nau, Bibl. Ges. Naturg., $2: 4$, p. 695 (1791). — Bkh., Naturg. Eur. Schmett, 5, p. 262 (1794). Schrank, Samml. Naturhist. Aufs., p. 169 (1796); id., , Faun. Boica, $2: 2$, p. 25 (1802). - Esp., Sohmett. in Abbild., 5 : 6, p. 150, t. 26, f. 1, 2 (1799). - Scharfenberg in Bchst. \& Scharfenb., Schädl. Forstins., 3, p. 692 (1805). - Haw., Iøep. Brit., 2, p. 312 (1809) (Geometra). - Lep. de St. F. \& Serv. in Encycl. Méth., 10, p. 88 (1825) (Phalaena). - Tr., Schmett. Eur., $5: 2$, p. 438 (1825) (Cab.); 6:1, p. 387 (1827) (Cab.). - Steph., Syst. Cat. Brit. Ins., 2, p. 127 (1829) (Cy.); id., MI. Haust., 3, p. p. 198 (1831) (E.). - Dup., Hist. Nat. Lép. Eur., $8: 1$, p. 40 , t. 172 , f. 7 (1830) (E.). - Wood, Index Ent., p. 87, t. 20, f. 527 (1835) (Phalaena). - Zell., Isis, 1838, p. 694 (1838) (Cab.). - H.-Sch. in Panz., Deutschl. Ins., 172, p. 102 (1840) (Idaea); id., Syst. Bearb. Schmett. Eur., 3:24, p. 31 (1847) (E.). - Westw. in Humphr. \& Westw., Brit. Moths, 2, p. 25, t. 61, f. 17 (1843?) (E.). - Eversm., Faun. Iep. Volg.Ural., p. 395 (1844) (Idrea). - Led., Verh. Zool.-Bot. Ve1: Wien, 3, p. 174 (sep. p. 10) (1853) (2.). - Kayser, Deutseh. Schmett., p. 477 , t. 134 , f. 2 (1857-58) (Z.). - Guen., Spee. Gén. Lép., 9, p. 412 (1858) (E.). Staint., Man. Brit. Butt. \& Moths, 2, p. 39, fig. (1858) (E.). - Heinem., Schmett. Deutschl., 1, p. 711 (1859) (2.). - Walk., List Lop. Ins., 22, p. 629 (1861) (E.). Newm., Brit. Moths, p. 73 (1869) (E.). Frey, Lep. Schweiz, p. 197 (1880) (Z.). Barr., Lop. Brit. Isl., 7, p. 316, t. 327, f.

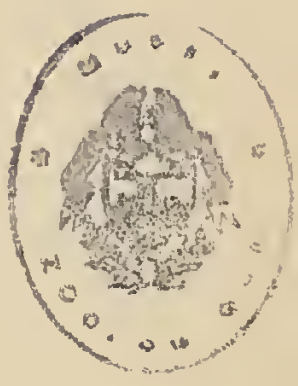


2-2 d (1901) (E.). - Oh. Oberth, Et. Lép. Comp., $12: 1$, p. 155 (1916) (E.).

circulifera [E. Geoffr.] Fourc. Ent. Paris., 2, p. 285 (1785).

denticulatu Thunb., D. D. Mus. Acad. Upsal., p. 75 (1788) (Suecia).

? p e nd ul $a$ ria Iang, Verz. Schmett., (ed. 2), p. 184 (1789) (err. det.).

a nn ulatum Reutti, Lep. Baden (ed. Mecss \& Spuler), p. 116 (1898) (Z.).

ab. of s o leta Riding, Ent. Rec., 10, p. 239 (1898); 11, p. 212, 289 (1899). - Tutt, Proc. Ent. Soc. Lond., 1898, p. 23 (1898). - Prout in Seitz, Macrolep., 4, p. 145 (1913). ab. biobs o le ta (Hellins, Ent. Mo. Mag.,

4, p. 181, 1868, s. n.) Riding, 1. c. (1898);

1. co (1899). - Tutt, I. c. (1898). - (Devon) Prout, l. c. (1913).

ab. s e rta ria Dannehl, Fnt. Zeits. (Frankf.), $40: 21$, p. 459 (1927).

gen. aest. a estiva Prout, l. c. (1913).

Morphologie: Bastelb., Iris, 13, p. 82, t. 2, f. 6 (1900). - Pierce, Genit. Geom. Brit., p. 37, t. 23 (1913).

B i olog i e: Réaumur, Mém. Hist. Ins., 2, p. 375 , t. 31 , f. 16 (1736). - LiAdmiral, Waarn. Verand. Ins., p. 8, t. 11 (1774). Schreiner, Zeits. Ges. Nat, 7, p. 244 (1856). - Porritt, Entom., 10, p. 137 (1877). -

O. Wilson, Larv. Brit. Lep., p. 102 (1880).

- Poulton, Tr. Ent. Soc. Lond., 1884, p. 38 seq., t. 1 , f. 9,10 (1884); id., l. c., 1892, p. 306 (1892); id., Philos. Tr. Roy. Soc., 178 B, p. 437 (1887). - Rühl, Soc. Ent., $8: 11$, p. 83 (1893). - E. Hofm.; Raup. Großschmett. Eur., p. 174 (1893); supp.-tab. 6, f. 14 (1910). - Buckler, Larv. Brit. Lep., 7, p. 63,173 , t. 115 , f. 8 (1897). - Riding, l. c. $(1898 / 99)$; id., Ent. Rec., 12, p. 80 (1900). - Barr., l. c. (1901).

hybr. s. n. (annulata of $\times$ pendularia H. J. Turn., Entorn., 46, p. 197 (1913) (ab.).

puppillaria Hb., Samml. Eur. Schmett., Geom., t. 13, Schweiz, f. 69 (1798) (Europa). - Prout in Seitz, SüdMacrolep., 4, p. 145, t. 40 (1913). Europa p u p ill laria [Charp.] Verz. Eur. Schmett., bis Syrien, p. 63 (1818) (Gcometra). - Tr., Schmett. Armenien, Eur., $5: 2$, p. 435 (1825) (Cab.); $6: 1$, p. Nond364 (1827) (Cab.). - Hb., Verz. , Bek. Afrika Schmett., p. 303 (1825) (Cod.). - Dup., Hist. Nat. Lép. Eur., 8:1, p. 30, t. 172, f. 2 (1830) (E.). - Bsd., Gen. et Ind. Meth., p. 220 (1840) (E.). - H.-Sch. in Panz., Deutschl. Ins., 172, p. 103 (1840) (Idaea); id., Syst. Bearb. Schmett. Eur., $3: 24$, p. 31 (1847) (E.). - II. Luc., Expl. 
Alg., Anim. Artic., 3, p. 394 (1849) (E.). Bertol., Nov. Comment. Bonon., 9, p. 165 (1849) (Cab.). - Zell., Stett. Ent. Zeit., 10, p. 309 (1849) (Geometra). - De la Harpe, Neue Denks. Schweiz. Ges. Nat. $13: 1$, p. 39 (1853); id., 1. e., $14: 7$, p. 6 (1854) (E.); id., Bull. Soc. Vaud., 6, p. $411(1860)$ (E.). - Led., Verh. Zool.-Bot. Ver. Wien, 3, p. 174 (sep. p. 10) (1853), 5, p. $211(1855)$ (Z.). - Guen., Spec. Gén. Lép., 9, p. 408 (1858) (E.). - Stdgl., Cat. Lep. Eur., p. 66 (1861) (Z.); (ed. 2) 1 p. 153 (1871) (\%.); (ed. 3) 1, p. 227 (1901) (E.); id., Hor. Soc. Ent. Ross., 7, p. 154 (1870) (Z.). - Millière, Ann. Soc. Linn. Lyon, 15, p .196 (Ic. 2, p. 288), t. 81, f. 9, 10 (1868) (E.); id., Mém. Soc. Sci. Nat. Cannes, 3, p. 186 (1873) (E.). - Berce, Faun. Ent. Franȩ., Lêp., 5, p. 115 (1873) (Ephyra). - Cuni y Martorell, Cat. Lep. Barcel., p. 134 (1874) (Z.). - Heyl., Tijd. Ent., 19, Verslag, p. 114 (1876) (Z.). Sand, Cat. Lép. Berry et Auv., p. 89 (1879) (Z.). - Frey, Lep. Schweiz, p. 197 (1880) (Z.). - Santos, Jorn. Sci. Lisboa, 10, p. 132 (1884) (E.). - Calberla, Iris, 3, p. 58 (1890) (Z.). - Meyr., Tr. Ent. Soc. Lond., 1892, p. 91 (1892) (L.). - Gmpbg., Nova Acta Ácad. Leop. Nat, 58 : 4, p. 288 (1892) (Z.). - Reb. \& Rogenh., Ann. Hofmus. Wien, 9, p. 72 (1894) (Z.). - Bastelb., Iris, 13, p. 82 (1900) (Z.). - Reb. in Spuler, Schmett. Eur. (E. Hofm., Großschmett. ed. 3) 2, p. 27, t. 58, f. 22 (1904); id., Berge's Schmetterlingsb., (ed. 9), p. 325 (1910) (Cod.). - Gatto, Faun. Lep. Malta, Eteroc., p. 21 (1905) (E.). - Ribbe, Lep.-Fauna Andal, (Iris, 23, Beih. 3/4), p. 311 (1912) (Cod.). - Vorbrodt \& Müll.Rutz, Schmett. Schweiz, $2: 1$, p. 28 (1913) (Cod.). - E. Turati, Atti Soc. Ital. Sci. Nat., 51, p. 326, t. 9, f. 11 (1913) (E.). Ch. Oberth., Et. Lép. Comp., $12: 1$, p. 151 (1916) (E.). - Culot, Noct. \& Géom. Eur., 3 , p. 88, t. 13 , f. 262,263 (1918) (E.). Schawerda, Iris, $35: 3 / 4$, p. 123 (1921) (E.). - Schwingenschuss \& Fr. Wagn. Zeits. Oesterr. Ent.-Ver., 12, p. 46 (1927) (E.). - Zerny, Iris, $41: 2$, p. 120 (1927); id., Eos, 3, p. 406 (1927). - Vorbrodt, Iris, 42 , p. 73 (1928) (Cod.). - Zingg \& Hoffmann, Mitt. Sohweir. Ent. Ges., $15: 11$, p. 462 (1933) (Cod.) (nee Brahm 1791).

porata Wernb., Beitr. Schmettkunde, 1, p. 225, 263 (1864) (Geom.). - Siepi, Cat. Lép. Bouches-du-Rhône, p. 105 (1905) (E.) (err. det., nec L.).

pupillarium Reutti, Lep. Baden (ed. Meess \& Spuler), p. 116 (1898) (Z.). 
pup ullaria Hulst, Tr. Amer. Ent. Soc. 23, p. 300 (1896).

ab. badiaria Stdgr., Cat. Lep. Eur., (ed. 2) 1, p. 153 (1871); (ed. 3) 1, p. 277 (1901). - Millière, 1. c., f. 12 (var. gyraria part.) (1868). - E. Turati, l. c., f. 12 (1913). - Prout, 1. c. (1913). - badiata Culot, t. c., p. 89, f. 264 (1918).

ab. gyrata Hb., Samml. Eur. Schmett., Geom.; t. 84, f. 434 (1814?); id., Verz. Bek. Schmett., p. 303 (Cod.). - Tr Schmett. Eur., 5:2, p. 435 (1825); 6:1, p. 364 (1827) (Cab.). - Stdgr., Cat. Lep. Eur., p. 66 (1861); (ed. 3) 1, p. 277 (1901); id. Hor. Soc. Ent. Ross., 7, p. 154 (1870). Cuni y Martorell, 1. c. (1874). - Vorbrodt \& Müll.-Rutz, t. c., p. 29 (1913). - Prout, l. c., t. 40 (1913). - Culot, t. c., p. 89 , t. 13 , f. 265, 266 (1918). - gyraria Hb.-Gey., Samml. Eur. Schmett., Geom., t. 104, f. 543 (1828). - Dup.y t. c., p. 31 , f. 3 (1830). - Bsd., 1. c., p. 220 (1840). - H.-Sch. in Panz, 1. c. $(1840)$; Syst. Bearb. Schmett. Eur., $6: 55$, p. 70 (1852). - Zell., Stett. Ent. Zeit., 10, p. 209 (1849). - Guend, t. c., p. 409 , No. $662 \mathrm{~A}$ et 663 (1858). Millière, l. c. f. 11 (1868); 1. e. (1873).

ab. nolaria Hb., Samml. Eur. Schmett. Geom., t. 63 , f. 327 (325 ex err.) (1800-08); id., Verz. Bek. Schmett., p. 302 (1825) (Cos.); id., Cat. Lép. Franck, p. 95 (1826) (Cy.) (France mérid.). - Bsd., 1. c. (1840). - Stclgr., 1. c. (1861); 1. c. (1901); id., Hor. Soc. Ent. Ross., 7, p. 155 (1870). Gmpbg., 1. c. (1892). - E. Turati, 1. c., f. 13 (1913). - Prout, 1. c., t. 40 (1913). (1913). - Ch. Oberth., Et. Lép. Comp., 12, p. 154 (1916). - Culot, t. c., p. 89, t. 13, f. 267 (1918). - mirtalis O. G. Costa, Cenni Zool., p. 74 (1835) (Aspilates) (Italia). - albipunctaria (Mann, M.S.) Heydenreich, Syst. Verz. Eur. Schmett., (ed. $3)$, p. 55 (1851) (Cab.) (Europa).

ab. simplex Th.-Mieg, Misa. Ent., 23, p. 51 (1916).

ab. a log a ria Schawerda, Zeits. Oesterr. Ent. Ver., 4, p. 67 (1919).

ab. scorteata Fr. Wagn., Verh. Zool-Bot. Ges. Wien, $74 / 75$, p. (101) (1926).

ab. fas ciat a Fr. Wagn., Zeits. Oesterr. Ent.Ver., 12, p. 46, fig. (1927).

(PyrénéesOr.)

(Pola)

(Dalmatien)

(Dalmatien)

var. (?) (gen. vern.) calaritan a Turati, Sardinien, Zeits. Wiss. Ins. Biol., 7, p. 210 (1911); id., Corsika, Atti Soc. Ital. Sci. Nat., $51: 3 / 4$, p. 326, t. 9, f. Capri 8 (1913) (Sardinia). - Schwingenschuss \& Fr. Wagn., Zeits. Oesterr. Ent.-Ver., 12, p. 46 (1927). - Sohn-Rethel, Iris, 43, p. 
15, t. 1, f. 19,20 (1929). — ab. badiaria E. T'urati, I. c.t f. 9 (1913). - ab. nolaria E. Turati, 1. c., f. 10 (1913).

Morphologie: Bastelb., 1. c. (1900). Pierce, Genit. Geom. Brit., p. 37 (1914).

B i o l o g i e: Millière, l. c. (1868). - E. Hofm., Raup. Großschmett. Eur., p. 175 (1893). Tutt, Ent. Rec., 11, p. 268 (1899).

lilacinipes Schaus \& Cockerell, Proc. Ent. Soc. Madeira, Wash., 25, p. 163 (1923) (praec. var.?). ? Azoren pu p illaria var. Beth.-Baker, Tr. Ent. Soc. Lond., 1891, p. 215 (1891) (Z.).

? p uppillaria form. Prout, Nov. Zool., 27, p. 271 (1920) (Azores).

maderensis Beth.-Baker, Tr. Ent. Soc. Lond., 1891, Madeira p. 216 (1891) (Z.). - Stdgr. \& Reb., Cat. Lep. Pal., 1, p. 278 (1901) (E.). - Warr., Nov. Zool., 12, p. 441 (1905). - Prout in Seitz, Macrolep., 4, p. 150, t. 5 c (1913).

ab. Wo llastoni Beth.-Baker, t. c., p. 215 (1891) (Acidalia). - Prout, 1. c. (1913).

ab. ir rufa $a^{-}$Warr., 1. c. (1905). - Prout, 1. c. (1913).

var. triline at a Prout in Seitz, Macrolep., Kanarische 4, p. 150 (1913) (maderensis ab.). - pu- Inseln pillario var.? Alph. in Romff., Mém. Iép., 5, p. 228 (1889). - Reb. \& Rogenh., Ann. Hofmus. Wien, 9, p. 72 (1894). - Reb., Ann. Hofmus. Wien, 11, p. 109 (1896); id., 1. c., 21, p. 32 (1906); id., 1. c, 31 , p. 39 (1917). - ab. latefasciata Prout, 1. c. (1913).

var. a zorensis Prout, Nov. Zool., 27, p. 271 (1920). - pupillaria Warr., Nov. Zool., 12 , p. 441 (1905) (err. det.).

B i o log i e: Beth.-Baker, 1. c. (1891).

porata $L$. Syst. Nat. (ed. 12), $1: 2$, p. 866 (1767) Süd- u. (Italia). - F., Syst. Ent., p. 631 (1775); Zentralid., Spec. Ins., 2, p. 286 (1781); id., Mant. Ins., 2, p. 200 (1787); id., Fnt. Syst., $3: 2$, p. 173 (1794). - Göze, Ent. Beytr., $3: 3$, p. 316 (1781). - do Villers, Linn. Ent., 2, p. 332 (1789). - Brez, Flore des Insectoph; p. 320 (1791) (prorata [err. typogr.]). Lasp. in Iil., Mag., 2, p. 161 (1803). Rossi, Faun. Etrusc. (ed. IIl.), 2, p. 310 (1808). - Steph., Ill. Haust., 3, p. 200 (1831) (E.). - Wood, Index Ent., p. 87, t. 20, f. 530 (1835) (Phalaena). - H.-Sch. in Panz., Deutschl. Ins., 172, p. 103 (1840) (Idaea). - Westw, in Humphr. \& Westw., Brit. Moths, 2, p. 25, t. 61, f. 21 (1843?) (E.). - Led, Verh. Zool.-Bot. Ver. Wien, 3, p. 175 (sep. p. 11) (1853) (Z.). - Helnem., Schmett. Deutschl., 1, p. 710 (Z.).

Europa

bis

Armenien 
Ad. Spr., Stett. Ent. Zoit., 24, p. 95 (1863) (Z.). - Snell., Vlind. Ned. Macrolep., p. 571 (1867) (Z.). - Newm., Brit. Moths, p. 72 (1869) (E.). - Stdgr., Cat. Lop. Eur., (ed. 2) 1, p. 153 (1871) (Z.); (ed. 3) 1, p. 277 (1901) (E.). - Berce, Faun. Ent. Franç., Lép., 5, p. 116 (1873) (E.). - Frey, Lep. Schweiz, p. 197 (1880) (Z.). - Ch. Auriv., Nord. Fjär., p. 206 (1891) (Z.). Gmpbg., Nova $\Lambda$ cta $\Lambda$ cad. Leop. Nat., $58: 4$, p. 285 (1892) (Leucophtalmia [sic]); $65: 3$, p. 403 , t. 18 , f. 81 (1896) (L.). - Meyr., Tr. Ent. Soc. Lond., 1892, p. 91 (1892); id., Handb. Brit. Lep., p. 245 (1895); id., Rev. Handb., p. 207 (1927) (L.). - Barr., Lep. Brit. Isl., 7, p. 304 , t. 328, f. $1-1 \mathrm{~d}$ (1901) (E.). - Reb. in Spuler, Schmett. Eur. (F. Hofm., Großschmett., od. 3) 2, p. 27, t. 58, f. 23 (1904) (Cod.); id., Berge's Sahmetterlingsb., (ed. 9), p. 325, t. 40, f. 8 (1910) (Cod.). - South, Moths Brit. Isl., 2 , p. 135 , t. 53 , f. 7,8 (1908). - Ribbe, Lep. Andal. (Iris 23, Beih. 3/4), p. 311 (1912) (Codonia). - Vorbrodt \& Müll.Rutz, Schmett. Schweiz, $2: 1$, p. 29 (1913) (Cod.). - Prout in Seitz, Macrolep., 4, p. 146 , t. 4 o (1913). - Klöcker, Danm. Fauna, 17 , p. 21 , t. 2 , f. 37 (1915) (E.). - Culot, Noot. \& Geom. Eur., 3, p. 90, t. 13, f. 268270 (191S) (E.). - Caradja, Mem. Sti. Acad. Romana, (3) $7: 8$, p. 27 (1931) (E.).

$p u n c t a r i a$ [Schiff.] Schmett. Wien, p. 107 (1775). - Hb., Samml. Eur. Schmett, Geom., t. 13, f. 67 (1798); id., Verz. Bek. Schmett., p. 303 (part? (1825) (Cod.). - Sepp, Besch. Wonder. Gods, 4, p. 31, t. 10 , f. 9 (1810-21) (s. ns); index (punctaria). C. S. Browne, Entom., 36, p. 308 (1903) (E.) (err. det., nec L.).

hӥbneraria Haw., Prodr. Lep. Brit., p. 24 (1802) (n. nud.); id., Lep. Brit., 2. p. 312 (1809) (Geometra).

o cellaria Haw., I. c. (1809) (Geometra). - Steph., Syst. Cat. Brit. Ins., 2, p. 127 (1829) (Cy.).

poraria Lep. de St. F. \& Serv. in Encycl. Méth., 10, p. 89 (1825) (Phalaena). - Tr.,

Schmett. Eur., $6: 1$, p. 356 (1827) (Cab.). Bsd., Gen. et Ind. Meth., p. 220 (1840) (E.). - H.-Sch., Syst. Bearb. Schmott. Eur., 3 : 24 , p. 31 (1847) (E.). - Zoll., Isis, 1847, p. 497 (1847) (Cab.). - H. Doubl., List Brit. Lep., p. 19 (1849) (E.). - Fologne, Ann. Soc. Ent. Bolg., 3, p. 126 (1857) (E.). Guen., Spoc. Gén. Lép., 9, p. 410 (1858) (:E.). - Walk., List Lep. Ins., 22, p. 626 (1861) (E.). - Millière, Mém. Soc. Sci. Nat. Cannes, 3, p. 186 (1873) (E.). - Ch. Oberth., Et. Lép. Comp., $12: 1$, p. 152 (1916) (E.). 
circularia Wernb., Beitr. Schmettkunde., 1, p. 433, 525; 2, p. 522 (1864) (Geometra) (err. det., nec F.).

poratum Reutti, Lop. Baden (ed. Meess \& Spuler), p. 116 (1898) (Z.).

ab. (gen. aest. pr. p.) vi \& peraria A. Fuchs, Stett. Ent. Zeit., 45, p. 266 (1884); id.,
Jahrb. Nass. Ver. Nat., 46, p. 93 (1893). Naufock, Jahresb. Wien. Ent. Ver., 12, p. 72 (1902). - F. Fuchs, Jahrb. Nass. Ver. Nat, 57, p. 40 (190t). - Vorbrodt \& Müll.-Rutz, 1. c. (1913). - Prout, 1. c., t. 40 (1913). Culot, 1. c., f. 271 (1918). - aestiva Hormuz., Verh. Zool.-Bot. Ges. Wien, 49, p. 39 (1899); 54, p. 4.40 (1904) (Bukowina).

ab. rubeuria Lambillion, Rev. Soc. Ent. Namur, 5, p. 250 (Cat. Lép. Belg. fasc. 16) (1905). - Prout, 1. c. (1913).

ab. $p u n c t u l a r i a$ Lambillion, l: c. (1905). - Prout, 1. c. (1913).

ab. linearia Lambillion, 1. c. (1905). Prout, 1. c. (1913).

ab. marginaria Lambillion, 1. c. (1905). Prout, 1. c. (1913).

ab. ve nat a Prout, t. c., p. 152 (1913). Gaul, Berl. Ent. Zeits, 51, S.B., p. 8 (1907) (s. n.) (Italien).

Morphologie: Bastelb., Iris, 13, p. 83, t. 3, t. 9, 14 (1900). - Pielce, Genit. Geom. Brit., p. 36 , t. 23 (1913).

B i ol o gie: Albin, Nat. Hist. Engl. Ins., t. 49 , f. a-d, t. 50 , f. a-d (1720). - Wilkes, Engl. Moths \& Butt., p. 37, t. 82 (1749). De Villers, Linn. Ent., 2, p. 653 (1789). IVilde, Pflanz. u. Raup. Deutschl., 2, p. 375 (1861). - Newm., Entom., 3, p. 355 (1867). - Redclyffe, Entom., 17, p. 280 (1884). Knatz, Jahresb. Ver. Nat. Cassel, 29/30, p. 85 (1884). - Riding, Ent. Rec., 4, p. 344 (1893); 12, p. 80 (1900). - E. Hofm., Raup. GroBschmett. Eur., p. 176 (1893); supp.-tab. 6, f. 15 (1910). - Barr., 1. c. (1901).

dataria Hulst, Eut. Amer., $2: 10$, p. 186 (1887) (Acidalia) (California); id., Tr. Amer. Ent. Soc., 23, p. 300 (1896) (L.). - Dyar, List Lep. N. Amer., p. 292 (1902). - Blackmore, Check-Iist Macrolep. Br. Col., p. 35 (1927).

piazzaria Wright, Ent. Nows, 35, p. 94 (1924).

(Wisperthal)

(Wisperthal) 
Ent. News, 6, p. 72 (1895) (E.); id., Tr. Amer.

Ent. Soc., 23, p. 300 (1896) (L.). - Dyar, List N. Amer. Lep., p. 292 (1902). - F. M. Jones, Ent. News, 21, p. 167 (1910). - Ch. Oberth., Et. Lép. Comp., 12, p. 150, t. 395, f. 3347 (1916) (Eph.). - Großbeck, Bull. Amer. Mus. Nat. Hist., 37, p. 89 (1917). - Forbos in Leonard, Ins. N. Y., p. 592 (1928).

? funginaria Gueu., Spec. Gén. Lóp., 9, p. 408 (1858) (Eph.) (s. loc.).

triseriata [Warr.] Hulst, Ent. News, 6, p. 72 (1895) (Eph.).

B i o log i e: Guen., l. c. (1858). - Goodell, Can. Ent., 9, p. 62 (1877).

culicaria Guen., Spec. Gén. Lép., 9, p. 407 (1858) Süd-Ost(E.) (Géorgie amér.). - Walk., List Lep. Ins., 22, p. 631 (1861) (E.). - Pack., Mon. Geom. U. S., p. 365 (1876) (E.). - Hulst, Ent. Amer., 2, p. 221 (1887) (E.); id., Ent. News, 6, p. 72 (1895) (E.); id., Tr. Amer. Ent. Soc., 23, p. 300 (1896) (L.). - Gmpbg., Nova Acta Acarl. Leop. Nat., 5S : 4, p. 291 (1892) (Z.). - Dyar, List Lep. N. Amer., p. 291 (1902). - Oh. Oberth., Et. Lép. Comp., 12 , p. 150 , t. 394 , f. 3345 (1916) ( $k$.$) .$

quercimontaria Bastelb., Stett. Ent. Zeit., $58: 1$, p. Holland, 120 (1897) (Z.) (Rheingau). - Riesen, Stett. Schweiz Ent. Zeit., $58: 3 / 4$, p. 322 (1898) (Z.). - bis Reb., Verh. Zool.-Bot. Ges. Wien, 48, SB., Herzegowina, p. (97) (1898) (Z.); id., Spuler's Schmett. NordEur. (E. Hofm., Großschmett., ed. 3) 2, p. Persion 28 , t. 71 a, f. 23 (1904) (Cod.); id., Ann. (var.?) Hofmus. Wien, 19, p. 255 (1904) (Cod.); id., Verh. Zool.-Bot. Ges. Wien, 58, SB., p. (78) (1908) (Cod.); id., Bergo's Sehmetterlingsb., (ed. 9), p. 325 (Cod.). - Bohatsch, Verh. Zool.-Bot. Ges. Wien, 48, SB., p. (170) (1898) (Z.). - A. Fuchs, Jahrb. Nass. Ver. Nat., 53, p. 50 (1900) (Z.). - Stdgr. \& Reb., Cat. Lep. Pal., 1, p. 277 (1901) (E.). - Naufock, Jahresb. Wien. Ent. Ver., 12, p. 72 (1902) (E.). - Rougemont, Cat. Lép. Jura, p. 172 (1903) (Z.). - F. Fuchs, Jahrb. Nass. Verh. Nat., 57, p. 40 (1904) (Cod.). Vorbrodt \& Müll.-Rutr, Schmett. Schweiz, $2: 1$, p. 30 (1913) (Cod.). - Prout in Seitz, Macrolep., 4, p. 147, t. 40 (1913). - Hoffmann \& Klos, Mitt. Nat. Ver. Steier., 53, p. 80 (1917) (Cod.). - Culot, Noct. \& G6om. Eur., 3, p. 91, t. 13, f. 272 , t. $1 \frac{1}{1}$, f. 273 (1918) (E.). - Strand, Archiv f. Naturg., 1919, A. 4, p. 5. - Meyer ot al., Stett. Ent. Zeit., 86, p. 104 (1925) (E.). - Heinrich, Deutsch. Ent. Zeits., 1928, p. 203 (192S). - Sterneck, Prodr., Faun. Böhm., p. 161 (1929) (E.) - Vorbrodt, Mitt. Schweiz. 
Ent. Ges., $14: 6$, p. 327 (1930) (Cod.). Lempke, Ent. Ber., 8, p. 462 (1933). - Deienne-Meyers, Lambill., 33, p. 70 (1933).

Mo 1. phologie: Bastelb., Iris, 13, p. 81, t. 2, f. 5, t. 3, f. 15 (1900). - Heydemann; Int. Ent. Zeits., $19: 47$, p. 369, fig. (1926); $22: 47$, p. 434 , fig. (1929).

B i o $l \circ$ g i e: Bastelb., l. c. (1897); t. c. : 2, p. 220 (1898)

ruficiliaria H.-Sch., Syst. Rearb. Schmett. Eur., Frankreich 6: 68, p. 135 (1855) (Z.). - Heinem., bis Crim Schmett. Deutschl., 1, p. 710 (1859) (Z.). - und Ad. Spr., Stett. Ent. Zeit., 21, p. 85 (1860), 23, p. 170 (1862) (Zonosoma). - Stigr., Cat. Lep. Fur. (ed. 2) 1, p. 153 (1871) ( $Z$. Kleinasien, punctaria ab.); (ed. 3) 1, p. 277 (1901) (E.). - Sand, Cat. Lép. Berry et Auv, p. 89 (1879) ( $Z$. punctroria ab:). - Calberla, Iris, 3, p. 60 (1890) (Z. punctaria ab.). Bastelb., Stett. Ent. Zoit., $58: 1 / 2$, p. 12 (1897); Ill. Ent. Zeits., 3, p. 257, 273 (1898) (Z.). - Favre, Faun. Macrolep. Vallais, p. 244 (1899) (Z.). - Gauckler in Krancher, Ent. Jahrb. 1900 (1901) (Z.). Reb. in Spuler, Schmett. Eur. (E. Hofm., Großschmett., exl. 3) 2, p. 28, t. $71 \mathrm{a}$, f. 24 (1904) (Cod.); id., Berge's Schmetterlingsb. led 9) p. 325 (1910) (Cod.) - Vorbrodt \& Müll.-Rutz, Schmett. Schweiz, $2: 1, \mathrm{p}$. 30 (1913) (Cod.). - Prout in Seitz, Macrolep., 4, p. 147, t. 40 (1913). - Culot, Noct. \& Géom. Eur., 3, p. ध3, t. 14, f. 278 (1918) (E.). - Andres \& Seitz, Senckenbergiana, 7, p. 61 (1925) (Cosymbria). - Derenne-Mejers, Lambill., 28, p. 71 (1928), 29, p. 2 (1929); id., Faun. Belg., Géom., p. 22 (1929). - J. Joann., Lambill., 28, p. 90 (1928). - Stauder, Ent. Anz., 9, p. 177 (1929) (Cod.). - Vorbrodt, Mitt. Schweiz. Ent. Ges., 14:6, p. 327 (1930) (Cod.).

? unilinear.ia Scharfenberg in Behst. \& Scharfenb., Naturg. Schädl. Ins., 3, p. 691 (1805) [Deutschland].

gy ra ria Tr., Schmett. Eur., $6: 1$, p. 366 (1827) (Cab.). - Zell., Isis, 1847, p. 498 (1847) (Cab.); id., Stett. Int. Zeit., 10, p. 209 (1849) [Geom.]. - De la Harpe, Neu. Denks. Schweiz. Ges. Nat., $13: 1$, p. 39 (1853) (Eph.) (err. det.).

pupillaria Zell., Isis, 1847, p. 498 (1847) (Cab.); id., ? Stett. Ent. Zeit., 10, p. 209 (1849) [Geom.] (err. det., nec puppillaria Hb.).

schaefferaria De la Harpe, Neu. Denks. Schweiz. Ges. Nat., $14: 7$, p. 5, f. 1 (1855); id., l. c., 20, p. 11 (1861) (Eph.) (Schweiz). - Stdgr., Cat. Lep. Eur., p. 189 (1861) (Z.). 
? suppunctaria Culot, Noct. \& Géom. Eur., 3, p. 92, t. 14, f. 277 (1918) (Eph.) (err. det.).

ab. ruberrima Bastelb., Ill. Zeits. Ent., 3, p. 274 (1898). - Prout in Seitz, Macrolep., 4, p. 147 (1913) (Ungarn).

ab. gen. vern. mattia cata Bastelb., l. c., p. 275 (1898). - Stdgr. \& Reb., Cat. Lep. Pal., 1 , p. 277 (1901). - Vorbrodt \& Müll.-Rutz, Schmett. Schweiz, 2:1, p. 31 (1913). E. Turati, Atti Soc. Ital. Sci. Nat., $51: 3 / 4$, p. 326 (1913). - Prout in Seitz, l. c., t. 4o (1913).

ab. gen. aest. privataria Bastelb., 1. c. (1898). - Stdgr. \& Reb., l. c. (1913). Vorbrodt \& Mülll.-Rutz, l. c. (1913). - E. Turati, l. c. (1913). - Prout, t. c., p. 148 (1913). - Culot, Noct. \& Géom. Eur., 3, p. 94, t. 14, f. 279 (1918). - privata Prout, t. c., t. 5 c (1913).

ab. gen. aest. cir cu mda ta ria Bastelb., 1. c., p. 274 (1898). - Prout, 1. c. (1913).

Morphol ogie: Bastelb., Iris, 13, p. 82, t. 2 , f. 7 (1900) (mattincata). - ? E. Petersen, Jona. Zeits. Nat., 47, p. 182 (1911).

B i o l o gie: Bastelb., Ill. Zeits. Ent., 3, p. 273 (1898).

punctaria L., Syst. Nat., (od. 10) 1, p. 522 (1758) (Finlandia); (ed.12), $1: 2$, p. 859 (1767); id., Faun. Suec., (ed. 2), p. 328 (1761). Clerck, Ic. Ins. Rar., 1, t. 5, f. 11 (1759). - O. F. Müll., Jaun. Fridr., p. 49 (1764); Zool. Dan. Prodr., p. 125 (1776). - Hufn., Berl. Mag., 4, p. 512 (1767). - F., Syst. Ent., p. 620 (1775); id., Spec. Ins., 2, p. 242 (1781); id., Mant. Ins., 3, p. 185 (1787); id., Ent. Syst., $3: 2$, p. 132 (1794). Knoch, Beytr. Ins., 1, t. 13, t. 1, f. 4-6 (1781). - Göze, Ent. Beytr., $3: 3$, p. 281 (1781). - De Villers, Linn. Ent., 2, p. 291 (1789). - Brahm, Ins,-Kral,, $2: 1$, p. 398 (1791). - Schwarz, Neu. Raupenkal., 2, p. 722 (1791). - Bkh., Naturg. Eur. Schmett., 5, p. 258 (1794). - Esp., Schmett. in Abbild., $5: 2$, p. 44, t. 6, f. $7-9$, ? t. 7 , f. 2 (1794). - Ill., Schmett. Wien (n. ed.), p. 424 (1801). - Lasp. in Ill., Mag., 2, p. 161 (1803). - Scharfenberg in Bchst, \& Scharfenb., Nat. Schädl. Forstins., 3, p. 690 (1805). - Bchst., Getr. Abbild. Nat. Gegenst., 6, p. 86, t. 97 (1805); id., Forstinseltol., p. 121 (1818). - Rossi, Faun. Etrusca (ed. Ill.) 2, p. 306 (1808). Haw., Lep. Brit,, 2, p. 312 (1809) (Geometra). - Sepp., Beschou. Wonder. Gods, 4, p. 29 , t. 10 , f. $1-8(1810-21)$ (s. n.); in-

Zentralu. SüdEuropa, südl. Skandinavien, Kleinasien bis NordPersien 
dex (linearia). - Guér. in Tabl. Encycl. et Méth., 18, p. 25, t. 88, f. 21 (1818) (Phalaena). - ? Hb., Verz. Bek. Schmett., p. 303 (part.) (1825) (Cod.) - Tr., Schmett. Eur., $5: 2$, p. 248 (1825) (Caß.); 6:1, p. $352(1827)$ (Cab.). - Lep. de St. F. \& Serv. in Encycl. Méth., 10, p. 91 (1825) (Phalaena). - Freyer, Beitr. Gesch. Eur. Schmett., 2, p. 17 , t. 54 , f. 1 (1828) (Cab.). - Steph., Syst. Cat. Brit. Ins., 2, p. 127 (Cy.); id., Ml. Haust., 3, p. 200 (1831) (E.). - Dup., Hist. Nat. Lén. Eur., $8: 1$, p. 25, t. 171, f. 8 (E.). - Hb.-Gey., Samml. Eur. Schmett., Geom., t. '109, f. 574 (1834) (Geometra). - Wood, Index Ent., p. 88, t. 20, p. 531 (1835) (Phalaena). - Thon, In- u. Ausl. Schmett., p. 195, t. 63 , f. 954,955 (1837). - Zell., Isis, 1838, p. 670, 1839, p. 455,1847 , p. 495 (Cab.). - Bsd., Gen. et Ind. Meth., p. 220 (1840) (E.). - Rtzb., Forstins., 2, p. 197 (1840) (Geometra). Westw. in Humphr. \& Westw., Brit. Moths, 2, p. 26, t. 6, f. 22 (1843?) (E.). - Eversm., Faun. Lep. Volg.-Ural., p. 394 (1844) (Cab.). - H.-Sch., Syst. Bearb. Schmett. Eur., $3: 8$, p. 3 (1844); $3: 24$, p. 30 (1847) (E.); $6: 68$, p. 135 (1855) (Z.). - Selys, Mém. Soc. Roy. Liege, 2, p. 18 (1845) (E.) - Bertol., Nov. Comment. Bonon., 9, p. 144 (1849) (Cab.). - Led., Verh. Zool.-Bot. Ver. Wion, 3, p. 175 (sep. p. 11) (1853) (Z.). - Kayser, Deutschl. Schmett., p. 477, t. 147, f. 1 (1857-58) (Z.). - Guen., Spec. Gén. Lép., 9, p. 410 (1858) (E.). - Fologne, Ann. Soc. Ent. Belg., 3, p. 126 (1859) (E.). - Heinem., Schmett. Deutschl., 1, p. 707 (1859) (Z.). - Walk., List Lep. Ins., 22, p. 626 (1861) (E.). Snell., Vind. Ned. Macrolep., p. 571 (1867) (Z.). - Gavere, Tijd. Ent., 10, p. 212 (1867) (Z.). - Longstaff, Ent. Mo. Mag., 4, p. 36 (1867) (E.) (ab.). - Noleken, Iep. Faun. Estl. (Arb! Nat. Ges. Riga) 1, p. 233 (1868) (Z.). - Newm., Brit. Moths, p. 72 (1869) (Ephyra). - Stdgr., Cat. Lep. Eur. (ed. 2) 1, p. I53 (1871) (Z.); (ed. 3) 1, p. 277 (1901) (E.). - Berce, Faun. Ent. Frane., Lép., 5, p. 117 (1873) (E.). - Christoph, Hor. Soc. Ent. Ross., 10, p. 39 (1873) (Z.). - Foucart, Cat. Lép. Douai, p. 58 (1876) (E.). - Curo, Bull. Soe. Ent. Ital., 10, p. 100 (1878) (Z.). - Frey, Lep. Schweiz, p. 197 (1880) (Z.). - Harrison, Naturalist, 1886, p. 18 (1886) (E.) (ab.). - Poppius, Acta Soc. F. F. Fonn., $3: 3$, p. 121 (1887) (Z.). - Ch. Auriv., Nord.' Fjär., p. 206 (1891) (Z.). - Gmpbg., Nova Aeta Acad. Leop. Nat., $58: 4$, p. 287 (1892); $65: 3$, t. 15, f. 98 (1896) (Z.) - Moyr., Tr. Ent. Soc. Lond., 1892, p. 91 (1892); id., Handb. 
Brit. Lep., p. 245 (1895); id., Rev. Handb., p. 207 (1907) (L.). - A. Fuchs, Jahrb. Ver. Nat., 46, p. 99 (1893), 53, p. 48 (1900) (Z.). - Caradja, Iris, $6: 2$, p. 230 (1894), 9, p. 68 (1896) (Z.). - Tutt, Brit. Moths, p. 247 , t. 11 , f. 5 (1896) (E.). - Hormuz., Verh. Zool.-Bot. Ges. Wien, 49, p. 39 (1899) (Z.). - Delahaye, Mém. Soc. Agric. Angers (5) 2, p. 179 (1900) (E.). - Fleck, Bull. Soc. Sci. Bucarest, 9:1, p. 157 (1900) (Z.) (àb.). - Barr., Iep. Brit. Isl., 7, p. 308, t. 326 , f. $2-2 \mathrm{f}$ (1901) (E.). - Naufock, Jahresb. Wien. Ent. Vor., 12, p. 72 (1902) (E.). - W. Potersen, Iep. Estl., p. 116 (1902); (ed. 2) 1, p. 230 (1924) (E.). Oudemans, Verh. Akad. Wet. Amsterdam, (2) $10: 1$, p. 43 , f. 25 (1903) (E.). - Hulme, Butt. \& Motlis Countryside, p. 233, t. 24, f. $203 \mathrm{~A}$ (1903) (E.). - Reb. in Spuler, Schmett. Eur., (E. Hofm., Großschmett., ed. 3) 2 , p. 28 , t. 58 , f. 24 (1904) (Cod.); id.', Berge's Schmetterlingsb., (ed. 9), p. 325 (1910) (Cod.). - South, Moths Brit. Isl., 2, p. 136 , t. 53 , f. 10,11 (1908) (E.). Ribbe, Iep.-Faun. Andal. (Iris 23, Beih. 3/4), p. 311 (1912) (Cod.). - Vorbrodt \& Müll.-Rutz, Schmett. Schweiz, $2: 1$, p. 30 (1913) (Cod.). - Prout in Seitz, Macrolep., 4 , p. 148 , t. 40 (1913). - Klöcker, Danm. Fauna, 17, p. 22, t. 2, f. 38 (1915) (E.). Hoffmann \& Klos, Mitt. Naturw. Ver. Steiermark, 53, p. '80 (1917) (Cod.). - Culot, Noct. \& Géom., Eur., 3, p. 91, t. 14, f. 274, 275 (1918). - Dannehl, Ent. Zeits. (Frankf.), $40: 21$, p. 459 (1927) (E.). - J. Joann., Lambill., 28, p. 90 (1928). - DerenneMeyers, Faun. Belg., Géom., p. 22 (1929).

te uto naria L., Syst. Nat., (ed. 10) 1, p. 823 (1758) (Europa). - Wrnbgl, Beitr. Schmettk., 1, p. 222 (tentonaria err. typogr.), 259, 260 (1864) (Geometra).

ful ta ria De Villers, Linn. Ent., 2, p. 330 (1789) (Gallia mer.).

a mataria Reichenbach, Bildorgallerie der Thierwelt, p. 17, t. 76, f. $7 \mathrm{a}, 7 \mathrm{~b}$ (1835) (Geometra) (err. det., nec L. 1761).

p u n ctata H.-Sch. in Panz., Deutschl. Ins., 172, p. 102 (1840) (Idaea).

a c utaria Roquette, Allg. Doutsch. Nat. Zeits. (n. s.), 3, p. 350 (1857) (Ennomos) (sine loc.).

p un ctarium Wllgr., Index Geom. et Noct. Scand., p. 22 (1874) (Z.). - Reutti, Lep. Baden (ed. Meess \& Spuler), p. 116 (1898) (Z.).

ab. co $m m$ unifas ciata Donov., Nat. Hist. Brit. Ins., 13, p. 49, t. 456 (1808) - Prout in Seitz, 1. c. (1913). - unifasciata Donov., l. c. (1808). 
ab. subangularia Haw., Lep. Brit., 2, p. 313 (1809). - Prout, 1. c. (1913).

ab. in fus c a ta E. Reuter, Länsi Suomi, 5, p. 76 (1890); id., Ent. Tidskr., 11, p. 202 (1890). - Prout, 1. c. (1913).

ab. arcufera. E. Reuter, Iänsi Suomi, 5, p. 77 (1890); id., Ent. Tidskr., 11, p. 202, f. 2 (1890). - Prout, 1. c. (1915).

ab. cing u la ta A. Fuchs, Jahrb. Nass. Ver. Nat., 53, p. 48 (1900). - Prout, t. c., p. 149 (1913). - Vorbrodt \& Müll-Rutz, Schmett. Schweiz, $2: 6 / 8$, p. 639 (1914).

ab. $p$ ulcherrimata A. Fuchs, 1. c. (1900).

- F. Fuchs, Jahrb. Nass. Ver. Nat., 57, p. 403 (1904). - Prout, 1. c. (1913). - H. W. Barker, Entom., 23, p. 237 (1890) (s. n.). - Barr., 1. c., f. 2 b (1901) (s. n.).

ab. demptaria A. Fuchs, t. c., p. 49 (1900). - Prout, 1. c. (1913).

ab. venata Prout, 1. c. (1913). - Barr., l. c., t. 2 e (1901) (s. n.).

(Bornich)

(Bornich)

ab. ochreifusa Prout, l. c. (1913). - (England) ab. erythrescens Preissecker, Verll. Zool.Bot. Ges. Wien, 72, p. (94) (1923).

gen. aest. na evat a Bastelb., Iris, 13, p. 83 (1900). - Warnecke, Verh. Ver. Nat. Unterh. Hamb., 14, p. 223 (1910). - Prout, t. c., p. 148 (1913). - Vorbrodt \& Müll.Rutz, l. c. (1914). - Esp., l. c., t. 7, f. 1 (1794) (s. n.). - South, 1. c., f. 12 (1908). - Culot, l. c., f. 276 (1918) (s. n.). - ab. foliata A. Fuchs, l. c., p. 49 (1900). Prout, 1. c. (1913). - ab. radiomarginata J. Joann., Bull. Soc. Ent. Fr., 1908, p. 45 (1908). - Prout, 1. c. (1913). - D'Aldin, Encycl. Ent., Lép., $3: 4$, p. 175 (1930).

Morphologie: Bastelb., Iris, 13, p. 83, t. 3, f. 10, 13 (1900). - IV. Petersen, Mém. Acad. Sci. St. Pét., (8) 9:6, p. 95 (1900). - Pierce, Genit. Geom. Brit., p. 36, t. 23 (1914).

Biologie: Réaum., Mém. Hist. Ins., 2, p. 365 , t. 29, f. 1-5 (1736). - Wilkes, Engl. Moths \& Butt., p. 37, t. 83 (1747). - L'Admiral, Waarn. Verand. Ins.s p. 2, to 2 (1774). - Kühn, Beschäft. Berl. Ges. Nat. Fr., 3, p. 33 (1777). - Knoch, 1. c. (1781). - Esp., 1. c. (1794). - Hb., Gesch. Eur. Schmett., Iarv., 5, Geom., 1, Ampliss. I. a. b, 1 f. - Sepp, 1. c. (1810-21). - Freyor, 1. c. (1828). - De Haan in Lyonet Anat. (Mém. Mus. Hist. Nat. Paris, 19) p. 454 , t. 26 , f. 18-23 (1830); id., Rech. Anat. Ins., p. 555 (1832). - Ad. Spr., Isis, 1839, p. 124 (1839). - Rtzb., 1. c. $(1840)$. - Roquette, l. c. (1857). - Wilde, Pflanz. u. 
Raup. Deutsohl., 2, p. 376, t. 10, f. 3 (1861). - Porritt, Ent. Mo. Mag., 8, p. 183 (1871). Cole, Entom., 8, p. 125 (1875); id., Proc. Ent. Soc. Lond., p. 6, 7 (1877); id., Tr. Epp. For: Field Club, 1, p. 10 (1881). - Praun, Abbild. Eur. Schmett-Raup., t. I, f. 6 (1875). - O. Wilson, Larv. Brit. Lep., p. 101, t. 20, f. 6 (1880). - Goossens, Ann. Soc. Ent. Fr., (6) 4, p. 9 (1884). - Buckell, Ent. Rec., 3, p. 253 (1892); 4, p. 243 (1893). - Merrifield, 'Ir. Lint. Soc. Lond., 1893, p. 65 (1893). - B. Hofm., Raup. Großschmett. Eur., p. 176, t. 40, f. 12 , t. 50 , f. 75 (1893). - Buckler, Larv. Brit. Butt. \& Moths, 7, p. 62, t. 115, f. 6 (1897). Barr., l. c. (1901).

suppunctaria Zell., Isis, 1847, p. 496 (1847) (Cab.) Italien,

(Italien); id., Verh. Zool-Bot. Ges. Wien, Spanien, 18, p. 586 (1868) (Z.). - Led., Verh. Zool.Bot. Ver. Wien, 3, p. 175, 259 (sep p. 11, 95) (1853) (Z. punctaria var.?). - Stdgr., Cat. Lep. Eur., p. 66 (1861) (Z.); (ed. 2) 1, p. 153 (1871) (Z. punctaria var.); (ed. 3) 1, p. 278 (1901) (E.); id., Hor. Soc. Ent. Ross., 7 , p. $155(1870), 14: 4$, p. $441(1879)$ ( $Z$. punclaria var.). - Ad. Spr., Stett. Ent. Zeit., 28, p. 73 (1867) (Z.). - Calberla, Iris, 3, p. 60 (1890) (Z. punctaria ab.). Hormuzaki, Verh. Zool.-Bot. Ges. Wien, 49, p. 39 (1899) (Z.). - Bastelb., Iris, 13, p. 85 (1900) (Z.). - ? Ribbe, Lep.-Faun. Andal. (Iris 23, Beih. 3/4), p. 312 (1912). - Prout in Seitz, Macrolep., 4, p. 149, t. 5 e (1913). - Rob., Sitrungsb. Akad. Wiss. Wien, Math.-Nat. Kl., 126, p. 787 (1917). Culot, Noct. \& Géom. Eur., 3, p. 92 (part.) (1918) (nec t. 14, f. 277) (E.). - ?Weiß, Treb. Mus. Ci. Nat. Barcel., $4: 2$, p. 84 (1920) (E.). - Stauder, Ent. Anz., 9, p. 177 (1929). - J. Joann., Amat. Pap., 4, p. 171 (1929). - Vorbrodt, Mitt. Schweiz. Ent. Ges., $14: 6$, p. 327 (1930) (Cod.).

subpunctaria H.-Sch., Syst. Bearb. Schmett. Eur., (3: 35, t. 67, f. 417, 1848, non binom.), $6: 55$, p. 69 (1852) (E.). - Guen., Spec. Gén. Lép., 9, p. 411 (1858) (E.). Beroe, Faun. Ent. Franç., Iép., 5, p. 117 (1873) (E. punctaria var.). - ? A. Fuchs, Jahrb. Nass. Ver. Nat., 42, p. 218 (1889) (Z. punctaria var.). - Favre, Faun. MacroLép. Vallais, p. 244 (1899) (Z. punctaria var.). - Reb. in Spule1', Schmott. Eur. (E. Hofm., Großschmett., ed. 3) 2, p. 28 (1904) (Cod.); id., Berge's Schmetterlingsb., (ed. 9), p. 326 (1910) (Cod.). - Vorbrodt \& Müll.Rutz, Schmett. Schweiz, 2:1, p. 31, (1913) (Cod.). - Ch. Oberth., Et. Lép. Comp., 12, p. $152(1916)(E$.$) .$

Süd-

Frankreich,

Schweiz,

Südost-

Europa,

Kleinasien 
ab. (?) amabilis Schawerda, Verh. Zool.Bot. Ges. Wien, 71, p. (159) (1922) (Cod.); 73, p. (164) f. 25 (1923) (Herzegowina).

Morphologie: Bastelb., 1. c., t. 3, f. 12 (1900).

linearia Hb., Sarnml. Eur. Sahmett., Geom., t. 13, Zentralf. 68 (1798); id., Verz. Bek. Schmett., p. Europa u. 304 (1825) (Adactylotis). - Haw., Lop. Brit., Nord2 , p. 314 (1809) (Geometra). - Tr., Italien Schmett. Eur., $5: 2$, p. 438 (1825) (Cab.). bis Nord- Fisch.-Rössl. in Thon's Archiv, 1:2, p. Persien 27 (1827) (Cab.). - Stdg1., Cat. Lep. Eur., (ed. 2), 1, p. 153 (1871) (Z.); (ed. 3) 1, p. $277^{\circ}$ (1901) (E.) - Ch. Auriv., Nond. Fjäril., p. 206 (1891) (Z.). - Gmpbg., Nova Acta Acad. Leop. Nat., $58: 4$, p. 289 (1892), 65 : 3, t. 15, f. 99 (1896) (Z.). - Hormuzaki, Verh. Zool.-Bot. Ges. Wien, 49, p. 39 (1899) (Z.). - Oudemans, Verh. Akad. Wet. Amsterdam, (2) $10: 1$, p. 43 (1903) (E.). Reb. in Spuler, Schmett. Eur. (E. Hofm., Großschmett., ed. 3) 2, p. 28 , t. 58, f. 25 (1904) (Cod.); id., Berge's Schmetterlingsb., (ed. 9), p. 326, t. 40, f. 10 (1910) (Cod.). South, Moths Brit. Isl., 2, p. 137, t. 53, f. 13, 14 (E.). - Ribbe, Lep.-Faun. Andal. (Iris, 23, Beih. 3/4), p. 311 (1912) (Cod.). Vorbrodt \& Müll.-Rutz, Schmett. Schweiz, 2 : 1. p. 31 (1913) (Cod.). - Prout in Seitz, Macrolep., 4, p. 149, t. $5 \mathrm{c}$ (1913). - Klöcker, Danm. Faun., 17, p. 22, t. 2, f. 39 (1915) (E.). - Culot, Noct. \& Géom. Eur., 3, p. 94, t. 14, f. 280,281 (1918) (E.). - Strand, Archiv f. Naturg., 1919, ^. 4, p. 5. Meyr., Rev. Inandb. Brit. Irep., p. 208 (1927) (L.). - Ghika, Int. Ent. Zeits., $21: 40$, p. 375 (1928) (Cod.) (ab.). - Osthelder, Schmett. Südbayerns (Mitt. Münchn. Ent. Ges., 19, Beilage), p. 395 (1929) (E.). Derenne-Meyers, Faun. Belg., Géom., p. 23 (1929).

? l ut e o laria De Villers, Linn. Ent., 2, p. 329 (1789) (Gallia mer.). - Wernb., Beitr. Schmettkunde., 2, p. 227 (1864) (Geometra). trilinearia Bkh., Naturg. Eur. Schmett., 5, p. 502 (1794) (nom. praeocc.) - Tr., Schmett. Eur., 6:1, p. 367 (1827) (Cab.). - Fisch.-Rössl. in Thon's Archiv, 1:2, p. 25 (1827) (non binom.). - Steph., Syst. Cat. Brit. Ins., 2, p. 127 (1829) (Cy.); id., III. Haust., 3, p. 200 (1831) (E.). - Dup., Hist. Nat. Iép. Eur., $8: 1$, p. 23, t. 171, f. 6, $? 7$ (1830) (E.). - Wood, Index Ent., p. 88 , t. 20, f. 532 (1835) (Phalaena). - H.Sch. in Panz., Deutschl. Ins., 172, p. 102 (1840) (Idaea); id., Syst. Bearb. Schmett. Eur., $3: 24$, p. 30 (1847) (E.); id., N. Schm. 
Eur., 1, p. 4, f. 31 (1856) (Z.). - Westw. in Humphr. \& Westw., Brit. Moths, 2, p. 26 , t. 61, f. 23 (1843?) (E.). - Bertol. Nov. Comment. Bonon., 9, p. 166 (1849) (Cab.). - Zell., Zeits. Ent. Bresl., 5 : 17, p. 66, t. $1,1.5$ (1851) (E.). - Ghiliani, Mem. Acad. Turin, (2) 14, p. 235 (1852) (E.). - Lod., Verh. Zool.-Bot. Ver. Wien, 3, p. 175 (sep. p. 11) (1853) (Z. tritinearia [err. typogr.]). - Boheman, Vet. Ak. Handl., 1851, p. 133 (1853) (Cab.). - De la Harpe, Neu. Denks. Scbweiz. Ges. Nat., $14: 7$, p. 5 (1855) (E.). - Guen., Spec. Gén. Lép., 9, p. 411 (1858) (E.). - Heinem., Schmett. Deutschl., 1, p. 709 (1859) (Z.). - Zebrawski, Motyl. Krakowa, p. 185 (1860) (Cab.). - W Walk., List Lep. Ins., 22, p. 628 (1861) (E.). - Snell., Vlind. Ned. Macrolep., p. 571 (1867) (Z.). - De Roo van Westmaas in Sepp, Ned. Ins., 2, p. 75, t. 17, f. 1-14 (1867?) (E.). - Frey. Lep. Sehweiz, p. 197 (1880) (Z.). - Meyr., Tr. Ent. Soc. Lond., 1892, p. 91 (1892); id., Handb. Brit. Lep., p. 245 (1895). - Oudemans, Tijd. Ent., 40, Verslag, p. 20 (1898) (Z.). Barr., Lep. Brit, Isl., 7, p. 312 , t. 327 , f. 1-1 f (1901) (E.). - Hulme, Butt. \& Moths Countryside, p. 231, t. 24, f. 203 (1903) (E.) (neo $\mathrm{Hb} . ; 1787$ ).

linearium Wllgr., Index Noct. et Geom. Scand. (Bil. Vet.-Ak. Handl., $2: 4$ ), p. 22 (1874) (2.). - Reutti, Lep. Baden (ed. Meess \& Spuler), p. 116 (1898) (Z.).

ab. carnearia (Brd., M.S.) De la Harpe, 1. c. (1855) (Schwëiz).

ab. nigrosparsaria A. Fuchs, Stett. Ent. Zeit., 62, p. 136 (1901). - Prout in Seitr, Macrolep., 4, p. 149 (1913) (Deutschland). ab. fas ciat a Prout, Entom. 45, p. 4, t. 1, f. 7 (1912); id. in Seitz, Macrolep,, 4, p. 150 (1913). - Cockayne, Proc. Ent. Soc. Lond., 1 p. 5 (1926) (England).

ab. infus c a ta (Barr., 1. c., f. 1 e, 1901, s. n.) Prout in Seitz, Macrolep., 4, p. 150 (1913). - Ch. Oberth., Et. Lép. Comp., 12, p. 153, t. 395 , f. 3350 (1916). - Culot, t. c., p. 95 , f. 286 (1918).

ab. de $m$ ptaria (Barr., 1. c., f. 1 a, s. n.) Prout, 1. c. (1913).

ab. a p p r o $x$ imans (Barr., 1. e., f. $1 \mathrm{~d}$, s. n.) Prout, 1. c. (1913).

ab. bicolor Ch. Oberth., Et. Lép. Comp., 12, p. 153, t. 395 , f. 3351 (1916). - Culot, 1. c., f. 285 (1918) (Osnabrück).

ab. ophthalmaria Cb. Oberth., t. c., p. 154, f. 3352 (1916). - Culot, t. c., p. 94, f. 282 (1918) (Sarthe). - ophtalmaria Osthelder, 1. c. (1929). 
ab. simplificaria Culot, t. c., p. 95, f. 283 (1918) (France).

ab. triline at a Schawerda, Verh. Zool.-Bot. Ges. Wien, 71, p. 159 (1922) (Mostar).

ab. mesoorthia V. G. M. Schultz, Beitr. Lippisch. Großschmett., p. 19 (1930). gen. aest. strabonaria Zell., Zeits. Ent. Bresl., 5 : 17, p. 66, t. 1, f. 4 (1851) (E.). - Guen., Spece. Gén. Lóp., 9, p. 411 (1858)

(E.). - Heinem., Schmett. Deutschl., 1, p. 709 (1859) (Z.). - Ad. Spr., Stett. Ent. Zeit., 21, p. 85 (1S60), 23, p. 170 (1862) (Z.). - Rössler, Wien. Ent. Monats., 5, p. 215 (1861) (trilinearia gen. aest. strabonaria). - Zell., Stett. Ent. Zeit., 28, p. 73 (1867). - H. Doubl., Entom., 5, p. 225 (1870). - Snell., Vlind. Ned. Microlep., p. I171 (1882). - Hormuzaki, 1. c. (1899). A. Fuchs, Stett. Ent. Zeit., 62, p. 137 (1901). - Vorbrodt \& Müll.-Rutz, 1. c. (1913). - Prout in Seitz, Macrolep., 4, p. 149, t. 5 c (1913). - Ch. Oberth., Et. Lép. Comp., 12, p. 152, t. 395, f. 3349 (1916). Culot,. Noct. \& Géom. Eur., 3, p. 95, t. 14, f. 284 (1918). - Stirand, Archir f. Naturg., 1919, A. 4, p. 5. - hybridaria Selys, Mém. Soc. Roy. Liège, 2, p. 18, (n. nud., punctaria var.) 34 (1845) (punctularia var.). - Fologne, Ann. Soc. Ent. Belg., 3, p. 126 (1859) (punctaria var.). - Derenne-Meyers, Lambill., 28, p. 72 (1928) 29, p. 3 (1929). - J. Joann., Lambill., 28, p. 90 (1928). ? suppunctaria Zell., Stett. Ent. Zeit., 28, p. 73 (1867).

Morphol og i e: Bastelb., Iris, 13, p. 85, t. 3, f. 11 (1900). - Cockayne, Proc. Ent. Soc. Lond., 1, p. 26 (1926).

B i ol o g i e: Ad. Spr., Isis, 1839, p. 124 (1839). - Newm., Entom., 2, p. 17 (1864). - De Roo van Westmaas, 1. ¿. (? 1867). - H. Fischer, Schrift. Naturw. Ver. Harz, 1, p. 35 (1886). - Oudemans, Tijd. Ent., 39, p. XXXIV, $8 \dot{8}$ (1896). - Barr., l. c. (1901). - Linden, Ann. Sci. Nat. Zool., (8) 14, p. 100, t. 15, f. 79 (1902). - Rougemont, Bull. Soc. Neuchatel Sci. Nat, 31, p. 14 (1903). - Vorbrodt, Tris, $42: 1$, p. 74 (1928). V. G. M. Schultz, Int. Ent. Zeits., $23: 39$, p. 445 (1930). - I. P. Robert, Les Chenilles, p. 325 , t. 51 (1931).

sympathica Alph., Hor. Soc. Ent. Ross,, 17, p. 166, Kuldscha, t. 8 , f. 76 (1883) (Timandra) (Kuldscha). Fergana, - Meyr., Tr. Ent. Soc. Lond., 1892, p. 91 (1892) (Calothysanis). - Prout in Seitz, Macrolep., 4, p. 151, t. 5 c (1913).

a lbilineata Stdgr., Iris, 5, p. 159 (1892)

(Zonosoma) (Margelan). 
dyschroa Prout, Ann. Mag. Nat. Hist., (9) 1, p. 25 Trinidad, (1918) (Trinidad).

Fr.-

Guayana,

Pará

anaisaria Schaus, Tr. Amer. Ent. Soc., 27, p. 255 Süd-Ost(1901) (huj. gen.?). Brasilien

angeronaria Warr., Nov. Zool., 2, p. 92 (1895) (S. Süd-OstAmerica).

Brasilien

carolina E. D. Jones, Proc. Zool. Soc. Lond., 1921, Parana p. 344, t. 3, f. 5 (1921) (Anisodes).

arthura Schaus, Tr. Amer. Ent. Soc., 27, p. 254 Sao Paulo (1901) (Craspedia).

stella Butl., Tr. Ent. Soc. Lond., 1881, p. 337 (1881) Guayana (Acidalia) (Amazon.).

bis Süd-

Brasilien,

Paraguay,

nubicolor Th.-Mieg, Le Nat., 14, p. 235

Ecuador

(1892) (Acidalia) (Cayenne).

go s in a Schaus, Tr. Amer. Ent. Soc., 27, p. 254 (1901) (Craspedia) (Parana).

semirosea Butt., Tr. Ent. Soc. Lond., 1882, p. 368 Chile (1882) (E.) (Valparaiso).

ab. notigera Butl., l. c. (1882).

umbrata Butl., l. c. (1882) (E.) (Valparaiso). Chile

nanaria Walk., List Lep. Ins., 22, p. 633 (1861) Große (Ephyra) (Jamaica). - Hampson, Ann. Mag. Antillen, Nat. Hist., (7) 7, p. 253 (1901) (Ephyra). Bahama- Prout, Tr. Ent. Soc. Lond,, 1910, p. 215 Inseln, (1910) (Euephyra).

n a n u la r i a H.-Sch., Corr.-Bl. Zool.-Min. Ver.

Dominica Regensb., 24, p. 180 (1870) (Z.) (Cuba). Gdl., Ent. Cubana, 1, p. 380 (1881) (Z.).

var. (?) serrulat a Pack., Rep. Peab. Acard. Südl. Sci., 5, p. 73 (1873) (Texas); id., Mon. Geom. 'U. S., p. 366 (1876) (Euephyra). Gmpbg., Nova Acta Acad. Leop. Nat., $58: 4$, p. 295 (1892) (Euephyra). - Hulst, Tr. Amer. Ent. Soc., 23, p. 300 (1896) (L.). Dyar, List N. Amer. Lep., p. 292 (1902). Prout, Tr. Ent. Soc. Lond., 1910, p. 215 (1910) (Euephyra). - Barnes \& Irc Dunnough, Check List Lep. Bor. Amer., p. 103 (1917).

o bscura Druce, Biol. Centr-Amer., Lep. Het., 2, p. 536, t. 99 , f. 6 (1898) (Mexico). toraria Schans, M.S. (in Mus. Tring) (Mexico).

Biologie: Cockerell, Ent. News, 11, p. 556 $(1900)$.

subsimilis Warr., Nov. Zool., 7, p. 153 (1900) West(Euephyra). 
coccaria H.-Sch., Cor.-Bl. Zool--Min. Ver. Regonsb., Mexiko, 24, p. 180 (1870) (Z.) (Venezuela).

Zentral-

Amerika,

Venezuela,

Ecuador,

c ons p i cillaria Druce, Biol. Centr.-Amer.,

Poru

Lep. Het., 2, p. 102 (1892) (E.) (orr. det., nec Snell.).

biline aria Schaus, Tr. Amer. Ent. Soc., 27, p. 254 (1901) (Craspedia) (Mexico).

obcoecaria Warr., M.S. (nom. nov. pro coecaria).

acutaria Walk., List Lep. Ins., 26, p. 1674 (1862) Columbien (E.) (Venezuela).

und

Venezuela

bis

Bolivien

und

Brasilien

conspicillaria Snell., Tijd. Ent., 17, p. 45 , t. 3 , f. 7 (1874) (Z.) (Columbien).

impudens Warr., Nov. Zool., 11, p. 487 (1904) Galapagos(Perixcra ?) (Gandner-Insel). - Schaus, Inseln Zoologica, 5 : 2, p. 28 (1923) /Perixera (Co34 symbia)) (huj. gen.?).

\section{Genus Anisodes Guen.}

Guen., Spec. Gén. Lép., 9, p. 415 (1858) (typus urcearia Guen.s Hampson sel., 1889). - Walk., List Lep. Ins., 22, p. 639 (1861). Moore, Lep. Ceyl.6 3, p. 443. (1887). - Hampson, Il. Het.2 9, p. 147 (1889); id., Faun. Ind., Moths, 3, p. 446 (1895). - Thurn., Proo. Linn. Soc. N. Sth. Wales, 32, p. 689 (1908), - Prout, Ins Samioa, $3: 3$, p. 124 (1928); id. in Seitz, Macrolep., 16, p. 54 (1933).

Pis or a ca Walk., List Lep. Ins., 24, p. 1079 (1862) (typus lyciscaria Guen.). - Turn., Proc. Linn. Soc. N. Sth. Wales, 32 , p. 694 (1908);

Z eugma Walk., List Leip. Ins., 26, p. 1622 (1862). Swinh., Lap. Het. Oxf. Mus., 2, p. 370 (1900).

Perixera Meyr., Ir. Ent. Soc. Lond., 1886, p. 209 (1886); id., Proc. Linn. Soc. N. Sth. Wales, (2) 2, p. 836 (1888). Turn., ibid., 32, p. 687 (1908) (typus prionodes Meyr.).

Streptopteron Swinh., Tr. Ent. Soc. Iond., 1892, p. 9 (1892) (typus posticamplum Swinh.),

Trirachopoda Hampson, Ill. Het., 9, p. 147 (1893) (typus subroseata Hampson, nee Walk.).

Mesotrophe Hampson, t. c., p. 148 (1893) (typus maximaria Hampson [intortaria Guen.]).

Streptopterum (Anisodes sect.) Hampson, Faun. Ind., Moths, 3, p. 450 (1895) (pro Streptopteron Swinh.).

Conchocomet a Warr., Nov. Zool., 2, p. 91 (1895) (typus sabulosa Warr.)d

Phrissos celes Warr., Nov. Zool., 3, p. 114 (1896) (typus argyromma Warr.). 
Platisodes Warr., 1. c. (1896) (indescr.) (pro jocosa Warr.).

Dizuga Warr., t. c., p. 372 (1896) (typus parva Warr.). - Turn., Proc. Roy. Soc. N. Sth. Wales, 32, p. 697 (1908).

Plocucha Warr., t. c., p. 376 (1896) (typus irregularis Warr.).

St ibarostoma Warr., t. c., p. 380 (1896) (typus griseata Warr.).

Brach y c o la Warr., op. cit., 4, p. 48 (1897) (typus absconditaria Walk.). - Turn., Proc. Linn. Soc. N. Sth. Wales, 32 , p. 683 (1908):

Pachuthalia Warr., t. c., p. 220 (1897) (typus rotundata Warr.).

Xenoprora Warr., t. c., p. 226 (1897) (typus parallela Warr.).

E m mesura Warr., op. cit., 5, p. 20 (1898) (typus semicompleta Walk.).

Phryssosceles (Anisodes sect.) Hampson, Journ. Bomb. Nat. Hist. Soc., 12, p. 86 (1898) (pro Phrissosceles Warr.).

Crys iplocia Warr., Nov. Zool., 9, p. 357 (1902) (typus pulvinaris Warr.).

Eremocentra Warr., op. cit, 10, p. 367 (1903) (typus flavareata Warr.).

Prostenodes Warr., t. c., p. 370 (1903) (typus glomerata Warr.).

Morphologie: Hampson, Faun. Ind., Moths, 3, p. 446450 (1895). - Turn., t. c., p. 637-638, 683-697 (1908). Prout, 1. c. (1928).

recusataria Walk., List Lep. Ins., 26, p. 1623 (1862) Borneo,

(Z.) (Sarawak). - Swinh., Cat. Lep. Het. Kedah,

Oxf. Mus., 2, p. 370 (1900) (Z.). Singapore,

Banka-

Insel

var. parva Warr., Nov. Zool., 3, p. 372 (1896) Queensland, (D.) (Queensland). - Turn., Proc. Linn. Luisiaden, Soc. N. Sth. Wales, 32, p. 697 (1908) (D.). Woodlark,

Salomons-

Inseln,

Dampier-

Insel,

? Amboina

glomerata Warr., Nova Zool., 10, p. 367 (1903) Holl.(Per.?) ㅇ (Brit.-Neuguinea).

u. Brit.-

or comosa Warr., t. c., p. 371 (1903)

Neuguinea (Prost.) (Brit.-Neuguivea).

ab. condens a ta Warr., t. c., p. 367 (1903) (Per.?).

rotundata Warr., Nov. Zool., 4, p. 221 (1897) Malayische (Pach.) (Penang). Halbinsel,

Sumatra

gigantula Warr., Nov. Zool., 11, p. 508 (1904) Ecuador (Carabaya).

bis

e oraria Ch. Oberth., Et. Lép. Comp., 20, p.

Bolivien 216, t. 549, f. 4635 (1923) (Peru). 
annularis C. Feld., Reise Novara, Lep. Het., 2, t. Brasilien, 127, f. 30, 30a (1875) (Brasilien). Süd-Ost-

Peru

rhodostigma Warr., Nov. Zool., 11, p. 31 (1904) Peru, (Bolivia).

Bolivien

aquila Schaus, Ann. Mag. Nat. Hist., (8) 10, p. Costa-Rica 292 (1912).

conferta Warr., Nov. Zool., 7, p. 141 (1900). Jamaika

fastidiosa Dogn., Ann. Soc. Ent. Belg., 44, p. Ecuador, $(1900)$ (Ecuador).

griseomixta Warr., Nov. Zool., 14, p. 212 (1907). Süd-OstPeru

parciscripta Warr., t. c., p. 213 (1907). Süd-Ost-

Peru

punctulosa Warr., Nov. Zool., 11, p. 31 (1904) Peru, (pintada ab.) (Bolivia). Bolivien

spatara Dogn., Ann. Soc. Ent. Belg., 44, p. 439 Loja, (1900).

? Bolivion

antennaria E. D. Jones, Proc. Zool. Soc. Iond., Süd-Ost1921, p. 345, t. 3, f. 7 (1921). Brasilien

concinnipicta Prout, Ann. Mag. Nat. Hist., (9) 1, Columbien p. 314 (1918) (A. (Pis.)).

sordida Dogn., Hét. Nouv. Amér. Sud, 1, p. 22 Nord-Peru (1910).

ab. nigridisca Dogn., t. c., p. 23 (1910).

ab. ta minat a Dogn., l. c. (1910).

iners Prout, Nov. Zool, 27, p. 271 (1920) (A. (Pis.)). Süd-Ost-

Peru

oöthesia Prout, t. c., p. 272 (1920) (A. (Pis.)). Ost-Peru

poliotaria Dyar, Proc. U. S. Nat. Mus., 44, p. 309 Mexiko (1913).

pomidiscata Warr., Nov. Zool., 11, p. 510 (1904) Süd-Ost(sequ. ab.?).

imparistigma Warr., t. c., p. 59 (1904). Süd-OstPeru

sypharioides Prout, Nov. Zool., 27, p. 272 (1920) Peru (A. (Pis.)).

maculidiscata Warr., Nov. Zool., 11, p. 509 (1904). Peru

mezclata Dogn., Ann. Soc. Ent. Belg., 37, p. 160 Columbien (1893) (Ecuador). bis Peru

decorata Warr., Nov. Zool., 11, p. 509 (1904) (pr. Süd-Ostf. vel syn.?).

Peru 
spiculifer Warr., Nov. Zool., 14, p. 216 (1907). Ost-Peru

scintillans Warr., Nov. Zool., 14, p. 215 (1907) Costa-Rica (Carabaya).

pruneliiaria (part.) Druce, Biol. Centr.Amer., Lep. Het., 2, p. 103 (1892) (err. det., nec H.-Sch.).

plenifasciata Dogn. (scintillans ab.), Mem. Soc. Ent. Belg., 18, p. 163 (1911) (Colombie).

silas Schaus, Ann. Mag. Nat. Hist., (8) 10, p. 294 Costa-Rica, (1912) (Costa-Rica) (pr. var. vel syn.?). Panama

connexa Warr., Nov. Zool., 14, p. 214 (1907) Ost-Peru, (radiata ab.). - Prout, Nov. Zool., $37: 2$, p. PEcuador 234 (1932) (Huanuco).

ab. radiata Warr., l. c. (1907) (nec Warr. 1897).

suberea Dogn., Ann. Soc. Ent. Belg., 44, p. 440 Ecuador $(1900)$.

bipartita Warr., Nov. Zool., 7, p. 141 (1900) (Po- Südpayan).

Columbien, Peru

major Dogn. (aequalipunctata var.) Hét. Nouv. Columbien, Amér. Sud, 3, p. 24 (1911) (sequ. f. ?). ? Ost-Peru

aequilipunctata Dogn., Ann. Soc. Ent. Belg., 45, p. Ecuador, 175 (1901) (Loja).

parcisquamata Prout, Ann. Mag. Nat. Hist., (8) 6, Ost-Peru p. 240 (1910) (pr. ab.?).

rubrannulata Prout, l. c. (1910).

West-

Columbien

plenistigma Warr., Nov. Zool., 8, p. 452 (1901). Panama

lancearia Feld., Reise Novara, Lep. Het., t. 126, Columbien f. 28 (1875) (Columbien).

ab. nigrinotata Dogn., Hét. Nouv. Amér. Bolivien Sud, 1, p. 21 (1910).

dulcicola Dogn. (radiata var.), Hét. Nouv. Amér. Columbien Sud, 3, p. 24 (1911).

?Ost-Peru

hetcrostigma Dogn., Mém. Soc. Ent. Belg., 19, p. Columbien 134 (1912).

rufistigma Warr., Nov. Zool., 11, p. 510 (1904). Carabaya

cndospila Prout, Nov. Zool., 27, p. 273 (1920). Carabaya

parvidens Warr., Nov. Zool., 14, p. 214 (1907). Ost-Peru

gracililinea Warr., t. c., p. 212 (1907). Carabaya

atrimacula Dogn., Mém. Soc. Ent. Belg., 18, p. Columbien 163 (1911) (Loja) (pr. ab.?).

Ost-Peru

hieroglyphica Warr., Nov. Zool., 11, p. 28 (1904). Peru 
spissata Warr., Nov. Zool., 7, p. 144 (1900) (Brit. Guayana, Guiana).

Amazonen-

$n$ u da ria Butl., Tr. Ent. Soc. Lond., 1881, strom p. 335 (1881) (err. det., nec Guen.).

timotheus Schaus, Ann. Mag. Nat. Hist., (8) 10, p. Costa-Rica 295 (1912).

bipunctata Warr., Nov. Zool., 11, p. 27 (1904). Carabaya

zeuctospila Prout, Nov. Zool., 27, p. 274 (1920) Amazonas, (A. (Pis.)).

stramineata Warr., Nov. Zool., 7, p. 145 (1900). Ficuador

calama Prout, Nor. Zool., 27, p. 274 (1920) (A. Rio (Pis.)) (pr. subsp.?).

difficilis Prout, t. c., p. 275 (1920) (A. (Pis.)) Amazon, (stramineata subsp.?). $\quad$ Ost-Peru

subviolescens Warr., Proc. U. S. Mus., 30, p. 431 Fr.(1906) (Fr. Guiana). Guayana,

Amazon

inaequalis Warr., Nov. Zool., 9, p. 521 (1902) (Pis.) Angola, (Barotse). - Prout in Seitz, Macrolep., 16, Nyasa, p. 54 (1933).

ab. obliterata Pront, 1. c. (1933).

ab. polystict a Prout, 1. c., t. $5 \mathrm{~g}$ (1933). Madagaskar

ab. \& ubapicata Prout, 1. c. (1933).

Ieonaria Walk., List Lep. Ins., 22, p. 635 (1861) Sierra-Leone

(Ephyma?) (Sierra Leone). - Butl., Tr. bis

Ent. Soc. Lond., 1881, p. 336 (1881). - Kamerun,

Swinh., Tr. Ent. Soc. Lond., 1904, p. 561 Sao Thomé

(1904) (Pis.). - Bastelb., Int. Ent. Zeits., 3, p. 101 (1909). (Pis.). - Strand, Arch. Nat., 78 A : 12 , p. 95 (1913) (Pis.); id., Ent. Rundsch., 35, p. 35 (1918) (Pis.). Prout, Tr. Ent. Soc. Lond., $1927: 1$, p. 189 (1927); id. in Seitz, Macrolep., 16, p. 54, t. $6 \mathrm{~d}(1933)$.

ab. (?) maculata Bastelb., 1. c. (1909) (Congo).

poeciloptera Prout, Nov. Zool., 27, p. 301 (1920) Sierra-Leone (A. (Pis.)) (Ivory Coast); id. in Seitz, bis Macrolep., 16, p. 54, t. 6 c (1933). Congo

lutearia Dewitz ( $\sigma$, nec O), Verh. Loop.r.Car. Akad., Elfenbein42 , p. 84 , t. 13 , f. 17 (1881) (Ephyra) Küste, (Lagos). - Prout, 1. c. (1920) (A. (Pis.i); Lagos id. in Seitz, t. c., p. $55, \mathrm{t} .5 \mathrm{~h}, 5 \mathrm{i}$ (1933).

Dewitzi Prout, 1. c. (1920) (A. (Pis.)); id. in Seitz, Elfenbein1. c., t. 5 i (1933).

lutearia o Dewitz, l. c., f. 21 (1881) Nigeria (Ephyra) (err. det., nec lutearia $\sigma^{7}$ ).

ab. transmuta Prout, t. c., p. 302 (1920);

Congo

id. in Seitz, l. c. (1933). 
paratropha Prout, 1. c. (1920); id. in Seitz, l. c. Usambara (1933).

lyciscaria Guen., Spec. Gén. Lép., 9, p. 406 (1858) Süd-Afrika, (Ephyra) (Namaqua). - Warr., Nov. Zool., Kenya, 2, p. 97 (1895) (Pis.). - Oh. Oberth., Et. Madagaskar Lép. Comp., 12, p. 150 , t. 394 , f. 3343 (1916) (Ephyra). - Prout in Seitz, 1. c. (1933).

b it a c ta ta Walk., List Lep. Ins., 24, p. 1079 (1862) (Pis.) (S. Africa).

ab. co e caria Guen., Spee. Gén. Lép., 9, p. 406 (1858) (Ephyra). - Oh. Oberth., Et. Lép. Comp., 12 , p. 150 , t. 395 , f. 3346 (1916). - Prout, Ann. Mag. Nat. Hist., (9) 1, p. 26 (1918) (Pis.); id. in Seitz, 1. e. (1933). - deremptaria Walk., List Lep. Ins., 26, p. 1580 (1862) (Anisodes). - demissaria Walk., t. c., p. 1603 (1862) (Acidalia?). - caecaria Ch. Oberth., Iit. Lép. Comp., $12: 2$, p. 21 (1916).

ab. sang u inata Warr., Nov. Zool., 11, p. 467 (1901) (Pis.) (Natal).

hirtifemur Prout, Nov. Zool., $37: 2$, p. 235 (1932); Südid. in Seitz, l. c., t. 6 d (1933). Seitz, I. c. (1933).

diplosticta Prout, Ann. Mag. Nat. Hist., (9) 1, p. Elfenbein24 (1918) (Pis.); id. in Seitz, 1. c., t. 6 d Küste, (1933) (Cameroons).

deremptaria Dewitz, Verh. Leop.-Car. Kamerun, Akad., 42 , p. 85 , t. 2, f. 20 (1881) (err. det, nee Walk.). - diremptria (err. transcr.) W. F. Kiirb., Zool. Rec., 18, p. Ins., 207 (1882).

metamorpha Prout, Tr. Ent. Soc. Lond., 1925, p. Madagaskar 311 , t. 36, f. $23(1925)$; id. in Seitz, l. c., t. $5 \mathrm{i}(1933)$.

insitiva Prout (Ent. Mitt. $3: 7 / 8$, p. 244, N. 42, Süd-Indien, s. 1l., 1914); id., Nov. Zool., 27, p. 275 (1920) Formosa (A. (Pis.)) (Nilgiris).

mesotoma Prout, Nov. Zool., 27, p. 276 (1920) Hainan, (A. (Pis.)) (Hainan); id., Bull. Hill Mus., Birma, $4: 1$, p. 130 (1930).

Malakka

punctata Warr., Nov. Zool., 4, p. 222 (1897) (Pis.). Nord- Turn., Proc. Linn. Soc. N. Sth. Wales, Queensland $32: 4$, p. 695 (1908) (Pis.).

auricosta Prout, Nov. Zool., 23, p. 19 (1916) Buru, (Ptochophyle?) (Goliath-Berg) (huj. gen.?). Holl.-

eos Prout, t. c., p. 20 (1916) (Ptochophyle?). Goliath-

Berg

geranium Prout, Nov. Zool., 24, p. 313 (1917) Holl.(Ptochophyle). 
compacta Warr., Nov. Zool., 5, p. 426 (1898) ( $\mathrm{Pi}$ - Kei-Inseln, soraca) (Key-Islands). - Prout, Ins. Sa- Neuguinea, moa, $3: 3$, p. 125 (1928).

Luisiaden,

Bismarck-

Archipel

var. (?) niveostilla nom. nov. - niveo- Nordpuncta Turn., Proc. Linn. Soc. N. Sth. Queensland Wales, $32: 4$, p. 694 (1908) (Pisoraca) (nec Warr. 1897).

var. la $u$ tokensis Prout, Ann. Mag. Nat. Fidji Hist., (10) 3, p. 595 (1929).

obstataria Walk., List Iep. Ins., 23, p. 769 (1861) (Acidalia) (Sarawak). - Swinh., Cat. Lep. Het. Oxf. MLus., 2, p. 363 (1900) (Pis.). Hampson, Faun. Ind., Moths, 3, p. 449 (1895); id., Journ. Bomb. Nat. Hist. Soc., 12 : 1, p. 86 (1898). - Strand, Iris, 1910, p. 199. - Rothsch., Journ. F. M. S. Mus., $8: 3$, p. 139 (1920).

a c u t a Moore, Lep. Ceyl., 3, p. 445, t. 215, f. 9 (1887) (Dikoya).

subroseata Hampson, Ill. Het., 9, p. 38 (1893) (T.) (Pundaluoya) (err. det., nec Walk.).

Pannulifera Meyr. in Gardiner, Faun. Mald. \& Laccad., 1 : 2, p.. 126 (1902) (Hulule) (err. det., nec anulifera Hampson). ab. ignorata Walk., List Lep. Ins., 26, p.

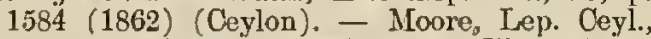
3, p. 445 (1887). - Hampson, Ill. Het., 9, p. 147 , ? t. 1699 , f. 2 (1893) (T.); id., Faun. Ind., Moths, 3, p. 449 (1895).

var. im $b$ ut a Warr., Nov. Zool., 4, p. 58 (1897) (Per.) (Khasia). - obstataria (part.) Swinh., Tr. Ent. Soc. Lond,, 1894, p. 178 (1894) (T.).

eryptorhodata Walk., List Lep. Ins., 26, p. 1682 Queensland, (1862) (Aspilates?) (Queenśland). - Turn., Proc. Linn. Soc. N. Sth. Wales, $32: 4$, p. 696 (1908); id., Tr. Roy. Soc. S. Austral., 46 , p. 270 (1922) (Pis.).

tenu is Warr., Nov. Zool., 14, p. 144 (1907) (Pis.) (Biagi) (var.?).

subdolaria Swinh., Proc. Zool. Soc. Lond., 1885, p. Indien, 856 (1885) (Bombay). Malay.-

simplex Warr., Nov. Zool., 10, p. 369 Halbinsel, (1903) (Pis.) (Aroa R.). - Turn., 1. c. Borneo, (1922) (Pis.).

decretaria Turn., Proc. Linn. Soc. N. Sth. Wales, $32: 4$, p. 697 (1908) (err. det., nec Walk.).

hirtipalpis Prout, Journ. F. M. S. Mus, $17: 1$, p. Kinabalu 52 , t. 10 , f. 11 (1932).

tricrista Prout, Nov. Zool., 32, p. 37 (1925). , : Neu-

Mindanao,

Brit.-

Neuguinea,

Queensland

mecklenburg 
sarawackaria Guen., Spece. Gén. Lép., 9, p. 420 Nond-

(1858) (Sarawak). - Ch. Oberth., Wt. Lép. Indien, Comp., 12, p. 158, t. 397, f. 3371 (1916) Perak, - J. Joann., Ann. Soc. Ent. Fr., 98, p. 481 Tonkin, (1929) (A. (Pis.)).

s a ra waka ria Walk., List Lep. Ins., 22, p. Formosa,

Borneo,

Ceram,

Neuguinea,

PNeumock-

lenburg

647 (1861).

lichenaria Swinh., Tr. Ent. Soc. Lond., 1892 , p. 8 (1892) (Khasia); id., Lep. Het. Oxf., Mus., 2, p. 369 (1900) (Per.). Hampson, Faun. Ind. Moths, 3, p. 450 (1895) (monetaria f.). - Prout, Ent. Mitt., 3, p. 244 (1914) (Pis.). - Candèze, Encycl. Lép., $2: 3 / 4$, p. 118 (1927).

o bs curata Warr., Proc. Zool. Soc. Lond., 1893 , p. 361 (1893) (Per.) (Sikkim).

rufiplaga Warr., Nov. Zool., 10, p. 367 (1903) (Mes.?); 14, p. 144 (1907) (Pis.) (Brit. New Guinea).

ab. stabilata Warr., Nov. Zool., 13, p. 91 (1906) (Per.); id., 1. c., 14, p. 144 (rufiplaga ab.) (1907) (Pis.).

ab. fasciata Warr., 1. c. (1907).

ab. nigriversa Warr., 1. c. (1907).

penumbrata Warr., Nov. Zool., 2, p. 97 (1895) Süd(Pis.). - Prout, Treubia, $7: 4$, p. 435 (1929). Celebes

var. a crobeles Prout, Bull. Hill Mus., 1: Buru, 2, p. 287 (1922) (Ceram); id., Treubia, Ceram l. c. $(1929)$.

ab. argyrostigma Prout, 1. c. (1922).

variospila Warr., Nov. Zool., 8, p. 24 (1901) (Pis.) Nord(India [Sikkim]) (sequ. var.?). - Prout, Indien, Ent. Mitt., 3, p. 244 (1914).

monetaria (part.) Hampson, Faun. Ind., Formosa Moths, 3, p. 450 (1895) (err. det., nec Guen.)!

falsareolaria o" Ch. Oberth. Et. Lép. Comp., $12: 1$, p. 156 (1916). - falseareolaria Ch. Oberth., op. cit., $12: 2$, p. 22, t. 396, f. 3367 (1916) (nec O) (Assam).

biformis Warr., M. S. in Mus. Tring (Pis.).

contrariata Walk., List Lep. Ins., 23, p. 770 (1861) Borneo, (Acidalia) (Sarawak). - Swinh., Cat. Lep. Malay.Het. Oxf. Mus., 2, p. 367 (obrinaria o) Halbinsel, (1900) (Per.).

Sumatra

patruelis Moore, Lep. Ceyl., 3, p. 444, t. 199, f. 10 Ceylon (1887). - Hampson, Journ. Bomb. Nat. Hist. Soc., $18: 1$, p. 51 (1907).

a b s c onditaria Hampson, II. Het., 9, p. 38 (1893) (Per.); id., Faun. Ind., Moths, 3, p. 447 (part.) (1895) (nec Walk.). 
subroseata Walk., List Lep. Ins., 26, p. 1583 (1862) Ceylon (hic pon.?).

minorata Warr., Nov. Zrool., 4, p. 48 (1897) (Br.) Kei- u. (Tenimber). - Prout, Nov. Zool., 32, p. 36 Tenimber(1925); id., Bull. Hill Mus., 4:1, p. 130 Inseln, (1930).

Hainan u.

Formosa

ephyrata Warr., Nov. Zool., 4, p. 60 (1897) (var.?)

(Ptochophyle) (Tenimber).

var. (? syn.) trib e le s Prout, Nov. Zool., 27, Sudest-

p. 276 (1920); id., 1. c., 32, p. 37 (1925). Insel,

P Nord-

Queensland

var. vinotincta Prout, Nov. Zool., 32, p. St.36 (1925).

Matthias-

Insel

discofera Swinh., Ann. Mag. Nat. Hist., (6) 14, Assam, p. 135 (1894) (Khasia). - J. Joann., Ann. Tonkin Soc. Ent. Fr., 98, p. 481 (1929) (A. (Pis.)). dis c ife ra Hampson, Faun. Ind., Moths, 3, p. 450 (1895). - Candéze, Encycl. Lép., $2: 3 / 4$, p. 118 (1927).

Iaiwana Wileman, Entom., 44, p. '30 (1911).

Formosa

alienaria Walk, List Lep. Ins., 26, p. 1586 (1862) (s. l.). - Prout, Nov. Zool., $37: 1$, p. 7 (1931); id., Journ. F. M. S. Mus., $17: 1$, p. 52 (1932).

Malay.-

Halbinsel,

Borneo,

? Siberut

maximaria Guen., Spec. Gén. Lép., 9, p. 419 (1858) (Borneo). - Walk., List Lep. Ins., 22, p. 646 (1861). - Swinh., Lep. Het. Oxf. Mus., 2, p. 369 (1900) (Mes.). - Ch. Oberth., Et. Lép. Comp., 12, p. 158, t. 396 , f. 3368 (1916). - ? J. Joann.s Ann. Soc. Ent. Fr., 98, p. 481 (1929) (A. (Meв.)).

immonstrata Walk., List Lep. Ins., 26, p. 1584 (1862) (Sarawak).

er ubescens. Warr., Nov. Zool., 3, p. 374 (1896) (Per.) (N. Borneo).

intortaria Guen., Spec. Gén. Lép., 9, p. 419 (1858) (Borneo). - Walk., List Lep. Ins., 22, p. 646 (1861). - ? Snell., Tijd. Ent., 24, p. 80 (1881). - Hampson, Ill. Het., 9, p. 38 (1893) (Mes.); id., Fuun. Ind., Moths 3, p. 450 (1895). - Swinh., Cat. Lep. Het. Oxf. Mus., 2, p. 369 (1900) (Mes.). - G. Semp., Reisen Philipp., (2) 6:6, p. 833

Borneo,

Singapore, ? Tonkin (1902) (Mes.). - A. Fuchs, Jahrb. Nass. Ver. Nat., 55, p. 84 (1902). - Oh. Oberth., Et. Lép. Comp., 12, p. 158, t. 396, f. 3369 (1916). - J. Joann., Ann. Soc. Ent. Fr., 98, p. 481 (1929) (A. (Mes.)).

expunctaria Walk., Journ. Linn. Soc. Zool., 3, p .194 (1859); id., List Lep. Ins.,

Ceylon,

Assam bis

Tonkin, Philippinen, Borneo, Sumatra, ? Colebes 
22, p. 645 (1861) (Singapore). - Swinh., Tr. Ent. Soc. Lond., 1894, p. 178 (1894) (Mes.).

responsaria Walk., List Iep. Ins., 22, p. 647 (1861) (Sarawak). - Swinh., Lep. Het. Oxf. Mus., 2, p. 369 (1900) (Pis.).

o visignata Moore. Lep. Ceyl., 3, p. 444 (1887) (Ceylon). - Butl., Proc. Zool. Soc. Lond., 1892, p. 132 (1892) (Mes.).

maximaria Hampson, IIl. Het., 9, p. 38 (1893) (Mles.); id., Faun. Ind., Moths, 3, p. 451 (1895) (err. det., nec Guen.).

nephelospila Meyr., Tr. Ent. Soc. Lond., 1889, p. Nouguinea, p. 487 (1889) (Per.) (Port Moresby). - AdmiraWarr., Nov. Zool., 3, p. 373 (1896) (M.). - litäts-, Turn., Proc. Linn. Soc. N. Sth. Wales, 32 : Salomons4, p. 694 (1908) (Pis.).

und

Luisiaden-

Inseln,

Nord-

Queensland

jocosa Warr., Nov. Zool., 3, p. 114 (1896) (Plat.?) Assam, (Khasia); id., l. c., 9, p. 355 (1902) (Br.). Pahang, - Hampson, Journ. Bomb. Nat. Hist. Soc., Nord-Öst$12: 1$, p. 86 (1898) (mometaria f.). - Prout, Sumatra Nov. Zool., 27, p. 302 (1920) (A. (B.)). var. clara Prout, l. c. (1920) (Br. New Neuguinea, Guinea).

Bismarck-, Trobriandu. D'Entrecasteaux-

Inseln

var. gly cidora Turn., Proc. Linn. Soc. N. NordSth. Wales, $32: 4$, p. 684 (1908) (Br.). Queensland

var. thysanopoda nom. nov. - fimbri- Salomonspedata Warr., Nov. Zool., 9, p. 355 (1902) Inseln (Br.) (Florida I.) (nec Walk., 1862).

cora Prout, Nov. Zool., 27, p. 303 (1920) (A. Holl.(Br.)) (Snow Mitns.).

Neuguinea

absconditaria Walk., List Iep. Ins., 26, p. 1580 Indien (1862) (S. Hindostan). - Hampson, Ill. bis Het., 8 , p. 29 , t. 156, f. 16 (1891); id., Formosa, Faun. Ind., Moths, 3, p. 447 (part.), ? f. Malay.203 (1895); id., Journ. Bomb. Nat. Hist. Halbinsel, Soc., $18: 1$, p. 51 (1907). - Swinh., Tr. ? Szechuan, Ent. Soc. Lond., 1894, p. 178 (1894); id., ? Mindanao, Cat. Lep. Het. Oxf. Mus., 2, p. 367 (1900) Java, (Per.). - Warr., Nov. Zool., 4, p. 48 (1897) (Br.). - Bastelb., Iris, 22, p. 173 (1909) (Br.). - Prout, Ent. Mitt., 3, p. 244 (1914) (Br.); id., Sar. Mus. Journ., $3: 2$, p. 174 (1926); id., Journ. Bomb. Nat. Hist. Soc., 31 : 1, p. 136 (1926). -. J. Joann., Ann. Soc. Ent. Fr., 98, p. 480 (1929) ( $A$. (Br.)i). 
? acritophyrta or West, Nov. Zool., 35 : 2, p. 254 (1930) (Mindanao) (err. det., nec typus).

clandestina Prout, Ann. Mag. Nat. Hist., (9) 2, Indien, p. 414 (1918) (A. (Br.); (Khasia). Ceylon, obrinaria (part.) Hampson, Faun. Ind., Malay.Moths, 3, p. 446 (1895) (err. det., nec Halbinsel, Guen.).

Borneo,

Java,

? Nias

paucinotata Warr., Nov. Zool., 8, p. 22 O (1901) Süd(Br.).

Celebes

niveopuncta Warr., Nov. Zool., 4, p. 48 o' (1897) Nord(Br.) (N. Queensland).

Australien, paucinotata 우 Warr., Nov. Zool., 8, p. Neuguinea, 22 (1901) (B.) („Australia's) (err. det.). Dampier-

obrinaria Turn., Proc. Linn. Soc. N. Sth. VulkanInsel, TVales, $32: 4$, p. 685 (1908) (Br.) (err. det., nec Guen.).

ab. indigens Warr., Nov. Zool., 13, p. 91 (1906) (? Per.) (Angabunga R.).

matthias Prout, Nov. Zool., 32, p. 37 (1925).

St.-

Matthias-

Insel

decolorata Warr., Nov. Zool., 4, p. 215 (1897) (Br.) (Lifu). - Prout, Ins. Samoa, 3:3, p. $124(1928)(A . \quad(B r)$.$) ; id., Tr. Ent.$ Soc. Lond., $77: 2$, p. 265 (1929) (A. $(B r))$.

Ioyalty-

Inseln,

Fidji,

Gresell-

schafts-

Inseln

posticamplum Swinh., Tr. Ent. Soc. Lond., 1892, Khasiberge p. 10, t. 1, f. 12 (1892) (Strept.).

p o st ic a m pla Hampson, Faun. Ind., Moths, 3, p. 450 (1895).

var. expunctor Prout, Journ. F. M. S. Kinabalu Mus, $17: 1$, p. 53 , t. 11, f. 17 (1932).

Warreni Dogn., Mém. Soc. Ent. Belg., 22, p. 9 Columbien (1913).

prunelliaria (Moritz, M. S.) H.-Sch., Samml. Außereur. Schmett., 1 , t. $\lceil 59]$, f. 329 (1855), p. 35 (s. n.) (1856), 81 (1858) (Zonosomai) (Venezuela). - Guen., Spec. Gén. Lep., 9 , p. 420 (1858). - Walk., List Lep. Ins., 22 , p. 642 (1861).

prunellaria H.-Sch., t. c., p. 61 (1858) (Zonosoma). - Dogn., Lép. Loja, 3, p. 75 (1894).

viator Prout, Nov. Zool., 27, p. 277 (1920) (Cara- Columbien, baya).

bis

Bolivien

Peru,

Bolivien

stricticata Warr., Proc. U. S. Mus., 30, p. 431 Mexiko, (1906) (Orizaba). 
Iateritiaria H.-Sch., Samml. Außereur. Schmett., 1, Venezuela, t. $[59]$, f. 332 (1855), p. 35 (1856) (Zono- Panama soma) (Venezuela). - Guen., Spec. Gén. bis Lép., 9, p. 488 (1858). - Walk., List Lep. Ins., 22, p. 642 (1861). - ? Möschl., Verh. Zool.-Bot. Ges. Wien, 31, p. 406 (1881) (Zonosoma). - ? Druce, Biol. Centr.-Amer., Lep. Het., 2, p. 103 (1892). - Dogn., I.ép. Loja, 3, p. 75 (1894). - Oh. Oberth., Et. Lép. Comp., 12, p. 157 (1916).

raspata Dogn., Ann. Soc. Ent. Belg., 44, p. 439 Ecuador (1900).

Dognini nom. nov.

$r u f i c$ e s Dogn., Mém. Soc. Ent. Belg., 22, p. 8 (1913) (magnidiscata var.) (Colombie) (nom. praeocc.)

seposita Prout, Nov. Zool., 29, p. 333 (1922) (Peru). Columbien

Bolivien,

?Mexiko,

?Surinam

Columbion,

Peru

bis

Bolivien

globaria Guen., Spec. Gén. Lep., 9, p. 417 (1858) (Colombie). - Walk., List Lep. Ins., 22, p. 642 (1861). - Oh. Oberth., Et. Lép. Comp., 12 , p. 157 , t. 396 , f. 3362 (1916).

metas pilata Walk., List Lep. Ins., 26, p. 1578 (1862) (Venezuela).

ordinata Walk., List Lep. Ins., 26, p. 1577 (1862). Jamaika,

? sypharia H.-Sch., Corr.-Bl. Zool.-Min. ? Cuba Ver. Regensb., 24, p. 181 (1870) (Zonosoma). - Gdl., Ent. Cubana, 1, p. 381 (Zonosoma) (err. det.).

co mplectaria Möschl., Abh. Senck. Ges., $14: 1$ (3), p. 70 (1886).

ab. congruaria Walk, t. e., p. 1578 (1862). - Schaus, Proc. Zool. Soc. Lond, 1896, p. 646 (1896).

ab. (?) a lbipupillat a Warr., Nor. Zool., 7, p. 141 (1900) (,S. America“).

flavidiscata Warr., Nov. Zool., 11, p. 28 (1904) Mexiko, (Vera Cruz) (pr. var.?).

¿globaria var. A. Guen,, Spec. Gén. Lép., 9, p. 417 (1858) (Brésil).

ruficeps Warr., Nov. Zool., 14, p. 215 (1907) (E. Columbien, Peru).

Columbien,

Venezuela

ab. in c e r t a Dogn., Mém. Soc. Ent. Belg., 18, p. 164 (1911) (Colombie).

sypharia Guen., Spec. Gén. Lép., 9, p. 416 (1858) Mexiko (Cayenne). - Walk., List Lep. Ins., 22, p. bis 641 (1861). - Dogn, Lép. Loja, 3, p. 75 (1894). - Ch. Oberth., Et. Lép. Comp., 12 , p. 156 , t. 396 , f. 3360 (1916).

fimbripedata Walk., List Lep. Ins., 26, p. 1579 (1862) (A.?) (Rio Janeiro).

? globaria (part.) Druce, Biol. Centr.Amer., Lep. Het., 2, p. 103 (1892) (err. det., nec Guen.).

Brasilien 
? binocellaria (part.) Druce, 1. c. (1892) (err. det., nec H.-Sch.)

? c a nfinaria (Walk. M. S.) Dogn., Ann. Soc. Ent. Belg., 44, p. 439 (1900) (indescr.).

binoceilaria H.-Sch., Samml. Außereur. Schmett., Venezuela. 1 , t. $[62\rceil$, f. 351 (1855); p. 36 (1856) Costa-Rica (Zonosoma) (Venezuela). - Walk., List Lep. bis Ins., 26, p. 1575 (1862) (Ephyra?). - Dogn., Bolivien Lép. Loja, 3, p. 75 (1894).

magnidis cata Warr., Nov, Zool., 11, p. 29 (1904) (Bolivia).

vineolincta Schaus, Ann. Mag. Nat. Hist., (8) 10, Costa-Rica p. 297 (1912) (praec. ab.?).

nivestrota Dogn., Ann. Soc. Ent. Belg., 57, p. 382 Panama, (1914). PColumbien

morbosa Dogn., Ann. Soc. Ent. Belg., 54, p. 113 Columbien (1910).

sopater Schaus, Ann. Mag. Nat. Hist., (8) 10, p. Costa-Rica 294 (1912).

subaeneseens Warr., Nov. Zool., 11, p. 31 (1904) Columbien, (Carabaya). Peru

graniilosa Dogn., Ann. Soc. Ent. Belg., 37, p. 159 Ecuador, (1893); Lép. Loja, 3, p. 75 (1894). ? Peru

aurantiata Warr., Nov. Zool., 11, p. 27 (1904) Carabaya, (Carabaya).
?Costa-Rica

? ros eigera Druce, Biol. Centr.-Amer., Lep. und Het., 2, p. 103 (1892) (Costa-Rica) (err. Panama det., nec Walk.).

ab. atridiscata Warr., Proc. U. S. Nat. Mus., 30, p. 428 (1906).

ignea Warr., Nov. Zool., 14, p. 212 (1907) (pr. f.?). Ost-Peru

tyehieus Schaus, Ann. Mag. Nat. Hist., (8) 10, p. Costa-Rica 296 (1912).

flavieornis Warr., Proc. U. S. Nat. Mus., 30, p. Carabaya 429 (1906).

ferruginala Warr., Nov. Zool., 7, p. 142 (1900). West-

Ecuador

rufulata Warr., Nov. Zool., 11, p. 510 (1904) (Ca- Costa-Rica rabaya).

bis

Bolivien

Uckendeni Prout, Nov. Zool., 27, j. 303 (1920). Columbien (Carabaya).

bis

Bolivien

pintada Dogn., Ann. Soc. Ent. Belg., 37, p. 159 Ecuador, (1893) (Loja); id., Lép. Loja, 3, p. 75 (1894). Peru

coenosata Warr., Nov. Zool., 14, p. 211 (1907). Ost-Peru

laseiata Dogn., Mém. Soc. Ent. Belg., 19, p. 134 Columbien (1912) (Cali).

aspera Warr., Nov. Zool., 8, p. 452 (1901) (Po- Columbien payan). 
mcdiolineata Warr., Nov. Zool., 11, p. 30 (1904). Carabaya brevipalpis Dogn., Mém. Soc. Ent. Belg., 22, p. 9 Bogota (1913).

khakiata Warr., Nov. Zool., 14, p. 213 (1907).

Ost-Peru

albidiscata Warr., Nov. Zool., 4, p. 438 (1857) Costa-Rica, (Euephyra) (Costa Rica). - Druce, Biol. Ost-Peru Centr.-Amer., Lep. Het., 2, p. 537 (1899) (Euephyra).

fulgurata Warr., Nov. Zool., 11, p. 508 (1904) (E. Peru).

llavistigma Warr., Nov. Zool., 14, p. 211 (1907). Paraguay

ocularis Warr., Nov. Zool., 7, p. 144 (1900) (Vene- Venezuela, zuela).

Fr.-

Guayana

costinotata Warr., t. c., p. 142 (1900) (Popayan). Costa-Rica bis

Bolivien

marginepunctata Dogn., Ann. Soc. Ent. Belg., 46, p. Ecuador 344 (1902) (= pr.).

illinaria Guen., Spec. Gén. Lép., 9, p. 416 (1858). Brasilien

palingenes Prout, Ann. Mag. Nat. Hist., (9) 11, Ost-Peru p. 308 (1923).

stigmatilinea Prout, Nov. Zool., 27, p. 278 (1920). Carabaya

lichenea Warr., Nov. Zool., 7, p. 143 (1900) (sequ. Jamaika ab.?).

urcearia Guen.. Spec. Gén. Lép., 9, p. 415 (1858) Jamaika, (Cayenne). - Walk., List Lep. Ins., 22, p. Mexiko 640 (1861). - Oh. Oberth., Et. Lép. Comp., bis 12 , p. 156 , t. 396 , f. 3358 (1916).

ordinata Walk., List Lep. Ins., 26, p.

Brasilien,

Paraguay 1579 (1862) (nec Walk., p. 1577) (Amazon). - Schaus, Proc. Zool. Soc. Lond., 1896, p. 646 (1896).

airectata Walk., t. c., p. I755 (1862) (nom. nov.).

ab. (?) importaria Möschl., Verh. Zool.Bot. Ges. Wien, 31, p. 407, t. 17, f. 17 (1881) (Surinam). - Druce, Biol. Centr:Amer., Lep. Het., 2, p. 104 (1892).

ab. $\operatorname{c}$ a $n$ d a ra Druee, "Biol. Centr.-Amer., Lep. Het., 2, p. 80, t. 4S, f. 26 (1892) (Bryoptera?! (Panama). - Dyar, Proc. U. S. Mus., 47, p. 231 (1914) (Anisodes).

ab. sylvia Druce, t. c., p. 539, t. 99 , f. 17 (1899) (Asthena) (Mexico).

diffusa Warr., Nov. Zool., 7, p. 146 (1900) (urcearia Guayana ab.) (Br. Guiana) (pr. ab.?).

maroniensis Dogn., Ann. Soc. Ent. Belg., p. 109 (1906) (Guyane franç.)

ab. (?) c e nt ra ta Dogn., Hét. Nouv. Amér. Sud., 1, p. 22 (1910) (Guyane franç.). 
dispergaria Möschl., Verh. Zool.-Bot. Ges. Wien, 31, Surinam, p. 406, t. 17, f. 15 (1881) (Zonosoma). ? Panama - ? Dyar, Proc. U. S. Nat. Mus., 47, p. 231

(1914) (Anisades) (urcearia ab.?).

superflua Warr., Nov. Zool., 4, p. 431 (1897) Venezuela (urcearia ab.?).

inquinata Dogn., Ann. Soc. Ent. Belg., 50, p. 110 Fr.(1906).

Guayana

Iantomaria Schaus, Tr. Amer. Ent. Soc., 27, p. 190 Venezuela (1900).

renifera Prout, Bull. Hill Mus., $1: 2$, 258 (1922) Br.- u. Fr.(Fr. Guiana); $1: 3$, t. 25, f. 7 (1924). Guayana

renistigma Prout, Ann. Mag. Nat. Hist., (8) 6, p. Chanchamayo 241 (1910); id., Bull. Hill Mus., 1 : 2, p. 258 (1922).

nigropustulata Warr., Nov. Zool., 7, p. 143 (1900) Venezuela (Tijuco). bis Fr.-

Guayana, Ecuador, Peru,

Brasilien

japaria E. D. Jones, Proc. Zool. Soc. Iond., 1921, Südostp. 344, t. 3, f. 6 (1921) (S. E. Brazil). Brasilien

bis

Guayana,

Bolivien,

Columbien

ruficosta Warr., Nov. Zool., 12, p. 45 (1905) (Br. Guatemala, Guiana). - Dyar, Proc. U. S. Mus., 47, p. Panama, 231 (1914).

Guayana

ochrieomata Warr., Nov. Zool., 11, p. 30 (1904). Nord-

Columbien

Ieucaniata Warr., Proc. U. S. Mus., 30, p. 430 Trinidad, (1906) (Br. Guiana).

Guayana

monera Schaus, Tr. Ámer. Ent. Soc., 27, p. 190 Mexiko, (1901) (Orizaba) (subpallida f.?). Tenezuela,

?Columbien

aguzala Dogn., Ann. Soc. Ent. Belg., 37, p. 160 Süd(1893) (Ephyra) (Loja); id., Lep. Loja, 3, Columbien, p. 75 (1894) (sequ. f.?).

Ecuador

subpallida Warr., Nov. Zool., 7, p. 145 (1900) (S. Mexiko, E. Brazil). ab. grisea Warr., 1. c. (1900) (subcarnearia Argentinien, ab).

ab. te nera Warr., 1. c. (1900).

Paraguay,

ab. stollaria Schaus, Tr. Amer. Ent. Soc. 27, p. 189 (1901). - figurata Warr., Nov. Zool., 12, p. 320 (1905). 
ab. potreria (Schaus, MS.) Warr., Proc.

U. S. Mus., 30, p. 430 (1906) (Mexico).

vuha Schaus, Proc. Ent. Soc. Wash., 31, p. 54, t. Süd4, f. 29 (1929). Brasilien

nudaria Guen., Spec. Gén. Lep., 9, p. 417 (1858). - Brasilien Walk., List Lep. Ins., 22, p. 641 (1861). Oh. Oberth., Et. Lép. Comp., 12, p. 156, t. 396, f. 3361 (1916).

terrens Warr., Proc. U. S. Mus., 30, p. 432 (1906). Mexiko

ignotaria Walk., List Lep. Ins., 26, p. 1576 (Ephy- Georgia u. $r a$ ?) (s. 1.) (sequ. f.?).

Florida

(Vereinigte

Staaten)

caducaria Möschl., Abh. Senck. Ges., $14: 3$, p. 70 Jamaika (1886).

subcarnearia Warr., Nov. Zool., 7, p. 145 (1900) Zentral- u. (S. E. Brazil) (pr. f.?). Süd-

Amerika

bis

Peru,

Brasilien

polysticta Prout, Nov. Zool., $37: 2$, p. 234 (1932). Costa-Rica, m ultipunctata Warr., Nov. Zool., 11, p. Columbien, (509) (1904) (S. E. Peru) (nom. praeocc., Peru nec Warr., 1899).

tolinta Schaus, Tr. Amer. Fnt. Soc., 27, p. 190 Süd-Ost(1901).

Brasilien

itinerans Prout, Nov. Zool., 29, p. 335

(1922).

proconcava Prout, Nov. Zool., $37: 2$, p. 235 (1932) Mexiko,

(Venezuela).

Costa-Rica,

Trinidad,

Venezuela,

Amazonen-

Strom

argenticristata Warr., Nov. Zool., 8, p. 452 (1901) Brasilien, (Sao Paulo). Paraguay

jonaria Schaus, Tr. Amer. Ent. Soc., 27, p. 190 Südost(1901).

Brasilien

megista Druce, Biol. Centr.-Amer., Lep. Het., 2, Mexiko p. 101, t. 51, f. 1 (1892) (Ephyra) (Guate- bis mala).

Ecuador,

Süd-Ost-

Peru,

?Cayenne

torsivena Warr., Nov. Zool., 11, p. 32 (1904). Bolivien,

Ecuador, ?Venezuela 
tharossa Druce, Biol. Centr.-Amer., Lep. Het., 2, Costa-Rica

p. 539 , t. 99, f. 16 (1899) (Asthena?) bis (Panama).

Brasilien

excavaria Schaus, Tr. Amer. Fnt. Soc., 27, p. 190 (1901) (S. E. Brazil).

bizaria E. D. Jones, Proc. Zool. Soc. Iond., 1921, p. Süd-Ost343 , t. 3, f. 3 (1921). Brasilien

Ilavipuncta Warr., Proc. U. S. Mus., 30, p. 429 Venezuela, (1906) (Br. Guiana). Br.-

Gr.-

spadix Prout, Nov. Zool., 29, p. 334 (1922).

Amazonenstrom

suspiciens Prout, t. c., p. 335 (1922).

Amazonenstrom

nebuligera Butl., Tr. Ent. Soc. Lond., 1881, p. Amazonen335 (1881) ơ (Rio Negro [,Napo" ex err.]). strom, coxaria Butl., 1. c., (1881) 웅. (err. det., Cayenne nee Guen.).

nodigera Butl., t. c., p. 334 (1881) (Amazon). Panama,

Columbien

bis

Surinam,

Amazonas,

Ecuador,

Peru,

Matto-Grosso.

coxaria Guen., Spec. Gén. Lep., 9, p. 416 (1858) (Cayenne). - Walk., List Lop., 22, p. 641 (1861). - Ch. Oberth., Et. Lép. Comp., 12, p. 156 , t. 396 , f. 3357 (1916). - ? Kaye \& Lamont, Cat. Trin. Lep. Het., p. 108 (1927).

Mexiko, ?Trinidad, Cayenne, Amazonenstrom, Süd-OstBrasilien

conifiniscripta Warr., Nov. Zool., 3, p. 374 (1896) Malay.(Per.) (Tenimber); id., l. c., 4, p. 394 Halbinsel, (1897).

p u n ct a t a Rothsch., Lep. Br. Orn. Un. Exp., p. 96 (1915) (Acidalia) (err. det, nec Warr., 1897 (Pis.)).

Borneo, Mindanao, Celebes, Sula, ab. (? var.) rubripuncta Warr., 1. c. Java bis 1897) (S.Celebes).

Tenimber, Kei-Inseln, Koer, Neuguinea

intermixtaria Swinh., Tr. Ent. Soc. Lond., 1892, p. Sikkim, 8 (1892) (Khasia); id., 1. c., 1894, p. 178 Assam, (1894). - Hampson, Faun. Ind., Moths, 3, Formosa p. 448 (1895); id., 1. c., 4, p. 563 (part.) (1896). 
interpulsata Walk., List Lep. Ins., 22, p. 642 (1861) Nord(India). - Hampson, Faun. Ind., Moths, 3, Indien, p. 4477 (1895) (part.). - Swinh., Cat. Malay.Lep. Het. Oxf. Mus., 2, p. 368 (1900) Halbinsel (part.). - Prout, Journ. F. M. S. Mus., (var.?), $17: 1$, p. $53(1932)$.

kinabalu

arenosaria Moore, Lep. Ceyl., 3, p. 445, t. 202, f. 1 Ceylon, (1887) (Dikoya). - Hampson, Hl. Het., 8, Indien, p. 29 (1891); 9, p. 38 (1893). - ? Swinh., Penang, Tr. Ent. Soc. Lond., 1894, p. 181 (1894) Siam(Craspedia).

Halbinsel

var. cretacea Warr., Nov. Zool., 5, p. 20 Neuguinea (1898) (Per.) (Woodlark I.). - lapidata bis Warr., op. cit., 6, p. 32 (1899) (Per.) (St. SalomonsAignan) (ab.). - ? Turn., Tr. Roy. Soc. S. Inseln, Austral., 46, p. 268 (1922) (part.) (Per.). ?Nond- venusta Warr., 1. c. (Per.) (St. Aignan) Queensland (ab.).

Curtisi Prout, Nov. Zool, 27, p. 303 (1920) Penang, (Penang).

Kedah

effeminala Prout, Ent. Mitt., 3, p. 294 (1914). Formosa

flavissima Warr., Nov. Zool., 14, p. 143 (1907) Kinabalu, (? Per.) (Biagi). - Prout, Juurn. F. M. Buru, S. Mus., $17: 1$, p. 53 (1932). Ceram, Holl.- $u$. Brit.-

ab. o phthalmicata Prout, Nov. Zool., 23, Neuguinea p. 25 (1916) (Dutch New Guinea).

acritophyrta West, Nov. Zool., 35:2, p. 254 (pars Luzon typ.) (1930).

radiata Warr., Nov. Zool., 4, p. 221 (1897) (?Per.). Celebes

frenaria Guen., Spec. Gén. Lép., 9, p. 421 (1858) Assam, (Borneo). - Walk., List Lep. Ins., 22, p. Malaya, 649 (1861). - Ch. Oberth., Et. Lép. Comp., Java, 12 , p. 158 , t. 397 , f. 3374 (1916).

pulverulenta Swinh., Tr. Ent. Soc. Iond., 1892, p. 9, t. 1, f. 8 '(1892) (Per.) (Khasia). - Hmpsn., Faun. Ind., Moths, 3, p. 448 (1895). - 'Turn., Proc. Linn. Soc. N. Sth. Wales, $32: 4$, p. 693 (1908); id., Tr. Roy. Soc. S. Austral., 46, p. 269 (1922).

pulverentula Swinh., op. cit., 1894, p. 247 (1894) (err. typogr.?).

ma cul ifera Swinh., Ann. Mag. Nat. Hist., (7) 6, p. 310 (1900) '(Per.; (Queensland).

$p l u m b$ eodis ca Warr., Nor. Zool., 10, p. 368 (1903) (Per.?) (Aroa R.).

c y clophora Turn., Proc. Linn. Soc. N. Sth. Wales, 32:4, p. 685 (1908) (Br.) (N. Queensland).

seriptata Walk., List Lep. Ins., 22, p. 650 (1861) Borneo, (A.?) (Borneo). - Swinh., Lep. Het. Oxf. Malay.Mus., 2, p. 369, t. 6, f. 20 (1900) (Pis.). Halbingel 
heydena Swinh., Tr. Ent. Soc. Lond., 1894, p. 178 Assam, (1894) (Cherrapunji). - Hmpsn., Faun. Ind., Tonkin Moths, 3, p. 449 (1895) (pr. var.?).

indecisa Warr., Nov. Zool., 14, p. 143 (1907) Holl.- u. (? Per.) (Biagi).

\section{Brit.-}

Neuguinea,

Ceram,

?Luzon

(var.)

obrinaria Guen., Spec. Gén. Lep., 9, p. 414 (1858) Ceylon, (Ephyra) (Coylon). - Walk., List Lep. Ins., 22, p. $639 \cdot(1861) \cdot($ Ephyra). - Frfld, Verh. Zool.-Bot. Ges. Wien, 17, p. 432, 448 (1867) (Zonosoma orbinaria) (err. typogr.). ? Hampson, III. Het., 9, p. 38 (1893) (Perixera); id., Faun. Ind., Moths, 3, p. 446 (part.) (1895). - Swinh., Tr. Ent. Soc. Lond., 1902, p. 663 (part.) (1902) (Per.). - ?? G. Semp., Reis. Philipp., (2) $6: 6$, p. 662 (1902) (Per.). - Ch. Oberth., Et. Lép. Comp., 12, p. 156, t. 395 , f. 3355 (1916) (Ephyra). - Prout, Ann. Mag. Nat. Hist., (9) 2, p. 415 (1918).

similaria Walk., List Lep. Ins., 26, p. 1582 (1862) 「Moulmein †. - Swinh., Tr. Ent. Soc. Lrond., 1890, p. 209 (1890). - Hampson, Journ. Bomb. Nat. Hist. Soc., $14: 4$, p. 652 (1903)

c ontractata Walk., t. c., p. 1585 (1862) (Sarawak).

ab. caligat a Walk., t. c., p. 1584 (1862) (Ceylon). - Moore, Lep. Ceyl., 3, p. 444, t. 199 , f. 4 (1887).

ab. nebulifera Warr., Nov. Zool., 5, p. 426 (1898) (Key Is.).

anulifera Hampson, IIl. Het., 9, p. 147, t. 169, f. Ceylon 15 (1593) (T.) (pr. ab.?). - annulifera Hampson, Faun. Ind., Moths 3, p. 448 (1895). - ?? G. Semp., t. c., p. 633 (1902) (nec Butl., 1889).

nebulosata Walk., List Lep. Ins., 26, p. 1583 (1862). - Moore, Lep. Ceyl., 3, p. 445, t. 199, f. 5 (1887). - Hampson, Ml. Het., 9 , p. 38 (1893) (Tri.); id., Faun. Ind, Moths, 3, p. 449 (1895) (obrinaria ab.?).

decretaria Walk., op. cit., 22, p. 650 (1862) (A.?) (Sarawak). - Swinh., Cat. Lep. Het. Oxf. Mus., 2, p. 368 (1900). - Prout, Sar. Mus. Journ., $3: 2$, p. 175 (1926); id., Journ. F. M. S. Mus., $17: 1$, p. 54 (1932) (obrinaria f.?).

p a l lida Moore, Lep. Ceyl., 3, p. 445, t. 201, f. 11 (1889). - Hampson, Faun. Ind. Moths, 3, p. 446 (1895) (obrinaria q-f.); 4, p. 563 (part.) (1896). - Plout, Ent. Mitt., 3, 244 (1914); id., Ann. Mag. Nat. Hist., (9) 2, p. 415 (1918). - J. Joann., Ann. Soc. Ent. Fr., 98, p. 480 (1929).
Burma,

Malay.-

Halbinsel,

Hainan,

Formosa,

Kei-Inseln
Ceylon

Ceylon, Malay.Halbinsel, Tonkin, Formosa, Borneo 
javensis Warr., Nov. Zool., 4, p. 216 (1897) (Conch.) Java, (pr. f.?).

?Sambawa

subsimilis Warr., t. c., p. 394 (1897) (Per.). Südœlebes

pyrrhocrica Prout, Sar. Mus. Journ., $3: 2$, p. 25, t. Borneo, 7, f. 36 (1926) (Borneo). Pahang,

Singapore

rubrisecta Warr., Nov. Zool., 5, p. 426 (1898) Kei-Inseln (Per.?) (vix obrinaria o-ab.?).

festiva Warr., Nov. Zool., 13, p. 90 (1906) (Per.?); Holl.- u. id., 1. c., 14 , p. 144 (1907) (Pis.) (Owen Brit.Stanley Range).

Neuguinea

subalbescens Warr., Nov. Zool., 10, p. 368 (1903) Brit.(Per.); id., 1. c., 14, p. 143 (1907) (Per.). Neuguinea

cxaucta Warr., Nov. Zool., 14, p. 143 (1907) (Per.) Holl.- u. (Biagi).

Brit.-

Neuguinea

tenuilinea Warr., Nov. Zool., 9, p. 359 (1902) (Per.) Guadalcanar, (Guadalcanar).

San

Christoval

recumbens Warr., t. c., p. 358 (1902) (P.er.) (Flo- Salomonsrida I.). Inseln

incumbens Prout, Nov. Zool., 27, p. 304 (1920). San

Christoval

epicoccastria Prout, 1. c. (1920).

Brit.-

Neuguinea

praetermissa Bastelb., Int. Ent. Zeits., 2, p. 38 ?Kei-Inseln, (1908) (Per.) (Fergusson-Insel). - Prout, Neuguinea Ins. Samoa, $3: 3$, p. 125 (1928).

bis

Salomons-

Inseln, ?Fidji

(var.?)

ampligutta Warr., Nov. Zool., 3, p. 376 (1896) Timor,

(Per. pallida ab.) (N. Queensland); id., 1. Nord-

c., 6, p. 336 (1899) (Per.). - Swinh., Tr. Queensland,

Ent. Soc. Lond., 1902, p. 663 (1902) (Per.). D'Entre-

casteaux

Luisiaden,

Bismarck-

Inseln

pilibrachia Prout, Nov. Zool., 27, p. 278 (1920) Brit.-

(Bougainville).

Neuguinea,

Dampier-

Insel,

Salomons-

Inseln 
sublanuginosa Warr., Nov. Zool., 10, p. 369 (1903) Brit.-

(Per.) (A roa R.).

Neuguinea,

Goodenough-

Insel

Turneri Prout, Nov. Zool., 27, p. 278 (1920) (A. Queensland, (Stib.)) (Queensland).

Neuguinea, pallida Turn., Proc. Linn. Soc. N. Sth.

Wales, 32, p. 691 (1908) (err. det., nec Moore).

Trobriand-,

Bismarck-

und

Salomons-

Inseln

griseala Warr., Nov. Zool., 3, p. 380 (1896) (Stib.) Süd-Indien, (N. Queensland). - Turn., t. c., p. 690 Formosa (part.?) (1908). bis Java, sy nt on a Meyr., Tr. Ent. Soc. Lond., 1897, Molukken, p. 72 (1897) (Per.) (err. det., nee Meyr. Nord1889).

Queensland und

Bismarck-

Inseln

furcata Warr., Nov. Zool., 3, p. 375 (1896) (Per.) Ceram, (Brit. Now Guinea); 10, p. 372 (1903) Nouguinea, (Stib.).

Ysabel-

Insel

longidiscata Warr., Nov. Zool., 11, p. 487 (1904) Queensland (Per.?).

pulverata Warr., Nov. Zool., 12, p. 424 (1905) (Stib.).

gris e ata (part.) Turn., 1. c. (1908) (nec Warr.).

leptopasta Turn., t. c., p. 691 (1908).

Nord-

Queensland

samoana Warr., Nov. Zool., 4, p. 216 (1897) (B.) Samoa,

(Upolo). - Prout, Ins. Samoa, 3:3, p. 125 Fidji,

(1928) (A. (X.)); id., Tr. Ent. Soc. Lond., Loyalty-

$77: 2$, p. 266 (1929) (A. (X.)). Inseln,

parallela Warr., t. c., p. 226 (1897) (X.) Gesell-

(Lifu). - Prout, t. c., p. 126 (1928) (var.?). schafts-

Inseln

(var.?)

illepidaria ? Guen., Spec. Gén. Lép., 9, p. 421 Nordindien

(1858) (una $Q$ condita) (Sarnwak). - bis

? Walk., List Lop. Ins., 22, p. 649 (1861). - Formosa,

G. Semp., Reisen Philipp., (2) $6: 6$, p. 632 Philippinen, (1902) (Emm.). - Swinh., Tr. Ent. Soc. Java u. Lond., 1902, p. 664 (1902) (Emm.). - Ch. Celebes

Oberth., Et. Lép. Comp., 12, p. 159, t. 397, f. 3375 (1916). - J. Joann., Ann. Soc. Ent. Fr., 98, p. 480 (1929) (A. (Emm.)). Prout, Bull. Hill Mus., 4:1, p. 130 (1930). s e micomple $t a$ Walk., List Lep. Ins., 22, p. 651 (1861) (A.?) (Sarawak). - Warr., Nov. Zool., 5, p. 20 (1898) (Emm.). - 
Swinh., Cat. Lop. Het. Oxf. Mus., 2, p. 595

(1900). - Prout, Sar. Mus. Journ., $3: 2$, p. 175 (1926).

im me moraria Walk., List Lep. Ins., 35, p. 1618 (1866) (Java).

strictaria Snell., Tijd. Ent., 24, p. 81,

t. 8, f. 7 (1881) (Celebes).

pulvinaris Warr., Nov. Zool., 9, p. 357 (1902) Vulkan-,

(Cryps.) (Guadalcanar).

Salomons-

Inseln

irregularis Warr., Nov. Zool., 3, p. 377 (1896) Humboldt(Ploc.). - Prout, Nov. Zool., $37: 2$, p. 234 Bai (1932) (A. (Ploc.)).

var. Rothschildi Prout, 1. c. (1932). - Schneeberge warreni Rothsch., Lep. Br. Orn. Un. Exp., (Holl.p. 96 (1915) (nom. praeocc., nec Dogn.). Neuguinea)

nigricosta Warr., Nov. Zool., 3, p. 382 (1896) Holl.- u. (Bardanes) (Humboldt Bay). Brit.-

Neuguinea

var. postposita Warr., Nov. Zool., 9, p. Salomons359 (1902) (Ploc.) (Guadaleanar). Inseln

cristata Warr., Nov. Zool., 6, p. 335 (1899) (Ploc.) Salomons(Tulagi).

Insoln,

Neu-

Pommern

bisecta Warr., Nov. Zool., 13, p. 90 (1906) (Per.?); Brit.14, p. 142 (1907) (Per.). Neuguinea

dimerites Prout, Journ. F. M. S. Mus., $17: 1$, p. Kinabalu 54 , t. 11, f. 10 (1932).

subrosea Warr., Nov. Zool., 13, p. 91 (1906) (Per.) Borneo, (Angabunga R.). - Prout, Sar. Mus. Journ. Ceram, $3: 2$, p. 174 (1926); id., Journ. F. M. S. Neuguinea Mus., $17: 1$, p. 54 (1932).

sordidata Warr., Nov. Zool., 3, p. 376 (1896) (Pis.) Singapore, (Humboldt Bay)

Kei-Inseln, gris ea Warr., Nov. Zool., 5, p. 427 (1898) Neuguinea, (Pis.?) (Kei-Inseln); 6, p. 336 (1899) (Per.). Sudest$s u b s i m i l i s$ Rothsch., Lep. Br. Orn. Un. Insel Exp., p. 96 (1915) (err. det., nec Warr. 1897 ).

var. (?) odontata Turn., Proc. Linn. Soc, NordN. Sth. Wales, 32, p. 688 (1908) (Per.). Queensland

pictimaculis Prout, Nov. Zool., $35: 1$, p. 63 (1929) Ceylon, (A. (Per.)) (Java).

Malay.-

Halbinsel,

PTonkin,

Borneo,

Java 
sublunata Swinh., Tx. Ent. Soc. Lond., 1904, p. 560 Elfenbein(1904) (Per.) (Aburi). - Prout in Seitz, Küste bis Macrolep., 16, p. 55, t. 6 d (1933). Nigeria

ab. ar e olaria Prout, 1. c. (1933).

ab. argentispila Prout, 1. c. (1933).

misella Prout, Nov. Zool., $37: 2$, p. 235 (1932) (nom. Nigeria nov.) (Nigeria); id. in Seitz, t. c., p. 56, t. $6 \mathrm{~d}$ (1933) (sequ. f.?).

inornat a Warr., Nov. Zool., 5, p. 241 (1898) (Pis.?); id., l. c., 6, p. 33 (1899) (Pis.) (nom. praeoces.).

ab. maculata Prout in Seitz, 1. c. (1933). ab. mediaria Prout in Seitz, l. c. (1933).

landanata Mab., Ann. Soc. Ent. Fr., 66, p. 227 Landana (1897). - Prout in Seitz, Macrolep., 16, p. 55 (1933).

la ud a nata Sharp, Zool. Rec., 34, p. Ins. 223 (1898).

orboculata Prout, Nov. Zool., 29, p. 335 (1922) (A. Madagaskàr (Per.)); id. in Seitz, t. c., p. 56, t. $6 \mathrm{e}$ (1933).

dotilla Swinh., Tr. Ent. Soc. Lond., 1894, p. 179 Assam, (1894) (Khasia). - Hampson, Faun. Ind. Penaug, Moths, 3, p. 448 (1895). - Prout, Bull. Hainan, Hill Mus., $4: 1$, p. 130 (1930). Celebes

faustina Prout, Nov. Zool., 27, p. 304 (1920) (A. Holl.- u. (Per.)) (Snow Mtns.).

Brit.-

Nouguinea

flavispila Warr., Nov. Zool., 3, p. 312 (1896) (Per.) Sikkim (Khasia). - Prout, Ins. Samoa, 3:3, p. 128 bis (1928) (Per.); id. in Bull. Hill Mus., 4:1, Hainan, p. 130 (1930); id., Nov. Zool., $37: 1$, p. 7 Hong-kong, (1931). - J. Joann., Ann. Soc. Ent. Fr., 98, Selangor, p. 480 (1929) (A. (Per.)). Mantawiin ter pul s ata (part.) Hampson, Faun. Ind., Inseln, Moths, 3, p. 447 (1895); id., Journ. Bomb. Borneo Nat. Hist. Soc., $12: 1$, p. 86 (1898) (nec (var.?), Walk.).

Sambawa u. Sumba (var.)

var. lophosceles Turn., Proc. Linn. Soc. Queensland, N. Sth. Wales, $32: 4$, p. 688 (1908) (Per.) Neuguinea, (Queensland). - Prout, Ins. Samoa, $3: 3$, p. Fergusson128 (1928) (Per.).

Insel

nepheloscia Prout, Stylops, 2, p. 26 (1933).

Buru

pauper Butl., Ann. Mag. Nat. Hist., (5) 20, p. 245 Neuguinea (1887) (Maleyta). - Prout, Ins. Samoa, bis $3: 3$, p. 125 (1928).

Salomons-

s yntona Meyr., Tr. Ent. Soc. Lond., 1889, Inseln p. 487 (1889) (Per.) (Port Moresby).

var. e gens Prout, Bull. Hill Mus., 1:2, p. Ceram 287 (1922).

var. c e ls a Prout, Nov. Zool., 33, p. 7 (1926). Neupommern 
prionodes Meyr., Tr. Ent. Soc. Lond., 1886, p. Fidji 209 (1886) "(Per.). - Prout, Ins. Samoa, $3: 3$, p. 125 (1928) (A. (Per.)).

perscripta Warr., Nov. Zool., 3, p. 326 (1896) (Per. Sikkim, pallida ab.) (Khasia). - Prout, Ent. Mitt., Khasiberge 3, p. 244 (1914) (pallida ab.).

thermosaria Walk., List Lep. Ins., 26, p. 1607 Malay.(1862) (Acidalia) (Sarawak). - Swinh., Cat. Halbinsel, Lep. Het. Oxf. Mus., 2, p. 369, t. 5, f. 22 Borneo, (1900) (Pis.).

m ult ipunctata Warr., Nov. Zool., 6, p. 336 (1899) (Per.) (Milne Bay). - Prout, Nov. Zool., 27, p. 305 (1920) (var.?).

Vulkan-

Insel,

Brit.-

Neuguinea

palirrhoea Prout, Nov. Zool., 27, p. 305 (1920) Salomons(A. (Per.)) (Vella Lavella). Inseln

obliviaria Walk., List Lep. Ins., 22, p. 643 (1861) Coylon (Ceylon). - Prout, Ann. Mag. Nat. Hist., bis (9) 2, p. 415 (1918); id., Ins. Samoa, 3:3, Samoa p. 127 (1928) (A. (Per.)); id., Journ. F. M. S. Mus., $17: 1$, p. 54 (1932).

suspicaria Snell., Tijd. Ent., 24, p. 80, t. 8, f. 6-6c (1881) (Celebes). - A. Pagenst., Jahrb. Nass. Ver. Nat, 41, p. 171 (1888) (suspiciarin). - Meyr., Tr. Ent. Soc. Lond., 1897, p. 172 (1897) (Per.).

obrinaria Moore, Lep. Ceyl., 3, p. 443, t. 199, f. 4 a (1887). - ? Meyr., Tr. Ent. Soc. Lond., 1889, p. 487 (1889) (Per.). Swinh., Tr. Ent. Soc. Lond., 1894, p. 178 (1894); ? id., Cat. Lep. Het. Oxf. Mus., 2, p. 367 (1900) (Per.): id., Tr. Ent. Soc. Lond., 1902, p. 663 (part.) (1902). (Per.). - Hampson, Faun. Ind., Moths, 3, p. 446 (part.) (1895) (err. det., nec Guen.).

ab. rufidorsata Warr., Nov. Zool., 3, p. 312 (1896) (Per.); id., l. c., 8, p. 24 (1901) (Phriss.) (Khasia). - rufannularia Warr., Nov. Zool., 4, p. 221 (1897) (Per.) (Samoa).

nesidica Prout, Nov, Zool., $37: 1$, p. 7 (1931) Mentawi(Mentawi Is.).

Inseln,

Langkawi-

Insel

roseofusa Warr., Nov. Zool., 3, p. 376 (1896) (Per.). Nord-

Borneo

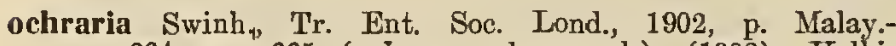
664 , p. 665 (ochracea, laps. cal.) (1902) Halbinsel, (Singapone) (sequ. f.?).

Banguey-

Insel,

Borneo,

Holl.-

Neuguinea 
argyromma Warr (Phriss.) (Khasia). - Hampson, Journ. Indien bis Bomb. Nat. Hist. Soc. $12: 1$, p. 86 (1898). Tonkin, - J. Joann., Ann. Soc. Ent. Fr., 98, p. Malay.480 (1929) (A. (Per.)). Halbinsel, manetaria Hampson, IIl. Het., 9, p. 30 Borneo, (1893) (Per.) (err. det.); id., Faun. Ind. Neuguinea, Moths, 3, p. 450 (part.) (1895) (err. det.). Neu-

g a et a Swinh., Tr. Ent. Soc. Lond., 1902, p. Pommern 665 (1902) (Borneo).

ab. heterospila Warr., Nov. Zool., 4, p. (Penang) 222 (1897). - areolaria 은 Guen., Spec. Gén. Lép., 9, p. 418 (1858) (err. det., nec o'). - falsareolaria $\$$ Ch. Oberth., Et. Lép. Comp., $12: 1$, p. 157; falscareolaria id., ibid., 2, p. 22 , t. 396 , f. 3366 (1916) (nec o').

hypocris Prout, Ins. Samoa, $3: 3$, p. 127 (1928). Samoa

argentosa Prout, Nov. Zool., 27, p. 278 (1920) (A. Ceylon, (Perr.)) (Borneo). - monetaria var. A Indien, Guen., Spec. Gén. Lép., 9, p. 418 (1858). - Malay.Ch. Oberth., Et. Lép. Comp., 12, p. 157, t. Halbinsel, 396 , f. 3364 (1916).

Sumatra,

Borneo,

Mindanao

monetaria Guen., Spec. Gén. Lép., 9, p. 458 (1858) Ceylon, (Borneo). - Walk., List Lep. Ins., 22, p. Nordindien, 646 (1861). - Warr., Nov. Zool., 8, p. 24 Malay.(1901). - Swinh., Tr. Ent. Soc. Lond., Halbinsel, 1902, p. 664 (1902) (Pis.). - Strand, Iris, Tonkin, 1910, p. 199. - Oh. Oberth., Et. Lép. Comp., Bornco 12 , p. 157 , t. 396 , f. 3363 (1916). - Prout, Nov. Zool., 27, p. 278, 279 (1920) (A. (Per.)); id., Sar. Mus. Journ., $3: 2$, p. 176 (1926). - J. Joann., Ann. Soc. Ent. Fr., 98, p. 481 (1929) (A. (Per.)).

ab. areolaria Guen., Spec. Gén. Ins., 9, p. 418 O $^{7}$ (nec Q) (1858). - Walk., List Lep. Ins., 22, p. 646 (1861). - Ch. Oberth., Et. Lép. Comp., 12, p. 157, t. 396, f. 3365 (1916). - Prout, Nov. Zool., 27, p. 279 (1920) (Borneo).

ab. argentispila Warr., Proc. Zool. Soc. Lond., 1893, p. 361 (1893). - Hampson, Faun. Ind., Moths, 3, p. 448 (part.) (1895). - Swinh., Cat. Lep. Het. Oxf. Mus., 2, p. 368 (1900). - Prout, l. co, (1920). - Joannis, l. c., (1929).

ab. hyperythra Swinh., Ann. Mag. Nat. Hist., (6) 14, p. 134 (1894). - Prout, 1. c. (1920); id., Sar. Mus. Journ., 3:2, p. 176 (1926) (Khasia).

ab. pleniluna Warr., Nov. Zool., 4, p. 394 (1897), 8, p. 24 (1901) (Per.?). - Prout, l. c. (1920) (Penang). 
va1. (?) homostola Meyr., Tr. Ent. Soc. Talaut Lond., 1897, p. 72 (1897) (Per.). - Swinh., Tr. Ent. Soc. Lond., 1902, p. 663 (1902) (Per.).

ceramis Meyr., Tr. Ent. Soc. Lond., 1886, p. 209 Coram, (1886) (Per.) (Solomon Is.). - Prout, 1. Saparoea, c. (1902) (monetaria c.).

le ucopelta Lower, Proc. "Linn. Soc. N. Sth. Wales, 23, p. 42 (1898) (Per.) (Queensland).

monetaria Turn., Proc. Linn. Soc. N. Sth. Wales, $32: 4$, p. 687 (1908) (err. det., nec Guen.).

ab. inornata Warr., Nov. Zool., 4, p. 216 (1897) (B.8). - Prout, Nov, Zool., $37: 2$, p. 235 (1932) (Banda Is.).

rudis Prout, Nov. Zool., 27, p. $305^{\circ}(1920)$.

Banda-

und

Kei-Inseln, Queensland,

Neuguinea

bis

Salomons-

Inseln

Holl.-

Neuguinea,

?Nond-

Borneo

sclota Turn., Proc. Linn. Soc. N. Sth. Wales, 32 : Nord-

4, p. 692 (1908) (N. Queensland). - Prout, Queensland,

1. c. $(1920)$

St. Aignan

subrubrala Warr., Nov. Zool., 12, p. 10 (1905) Guizo, (? Mes.) (Guizo). Neuab. $u$ s tipenn $i$ s Warr., t. c., p. 423 (1905) Georgien (Per.) (New Georgia).

sabulosa Warr., Nov. Zooł., 2, p. 91 (1895) (Conch.) Java, (Palawan [err. pro Palabuan]). Sarawak, Luzon

obviata Prout, Nov. Zool., $37: 2$, p. 235 (1932) Ceram, (Biagi). stram ineata Warr., Nov. Zool., 14, p. 141 (1907) (Eremocentra).

argyromma Rothsch., Lep. Br. Orn. Un. Holl.- u. Exp., p. 96 (1915) (A. (Phriss.)) (err. det.) (ab.).

panlophyrta Prout, 1. c. (1932).

Brit.-

Neuguinea

Brit.-

Neuguinea

flavirubra Warr., Nov. Zool., 3, p. 375 (1896) Nord(Per.?) (N. Queensland). - Turn., Tr. Roy. Queensland, Soc. S. Austral., 46, p. 268 (1922) (Per.). Neuguinea, niveopuncta O Warr., Nov. Zool., 4, p. Dampier48 (1897) (B.) (err. det., nec $\sigma^{7}$ ).

flavareata Warr., Nov. Zool, 10, p. 367 (1903) (Erem.) (nec. typ.).

u. AdmiralitätsInseln, ab. $t r a n s v e r s a t a$ Warr., t. c., p. 58 (1897) Luisiaden (Per.) (Queensland).

var. (? syn.) flavareata Warr., t. c., p. Malay.215 (1897) (Br.) (Penang); id., 1. c., 10, Halbinsel, p. 367 (part.) (1903) (Erem.). 
denticulata Hampson, Faun. Ind. Moths, 3, p. 447 Sikkim, (1895) (Naga). - Prout, Journ. F. M. S. Assam, Mus., $17: 1$, p. 55 (1932).

? monetaria Swinh., Cat. Lep. Het. Oxf. Kinabalu, Mus., 2, p. 369 (1900).

ab. mediusta Warr., Nov. Zool., 3, p. 114 ?Ceylon, (1896) 'Per.) (Khasia). - ? Bastelb., Iris, Ceram 22, p. 173 (1909) (Br.). (var.?)

var. (? syn.) glareosa Warr., Nov. Zool., Holl.-

14, p. 141 (1907) (Erem.) (Biagi). u. Brit.-

ab. fuscisecta Warr., l. c. (1907) (? = Neuguinea ab. mediusta).

porphyropis Meyr., Proc. Linn. Soc. N. Sth. Wales, Neu-

(2) 2, p. 837 (1888) (Per.) (N. Sth. Wales). südwales, - Peth.-Baker, Proc. Zool. Soc. Lond., Queensland, 1905 : 1, p. 94, (1905). - Turn., Proc. Linn. PFidji Soc. N. Sth. Wales, $32: 4$, p. 686 (1908) (Br.).

microsticta Turn., Tr. Roy. Soc. S. Austral., 42, Lordp. $278(1918)(B r . ?)$ (hic pon.?).

insigniata Warr., Nov. Zool., 7, p. 143 (1900) (Brit. Columbien Guiana). ab. li po se ma Dogn., Mém. Soc. Ent. Belg., Cayenne, 18, p. 164 (1911) (Guyane fr.). Peru,

Bolivien, Süd-OstBrasilien

castraria Schaus, Tr. Amer. Ent. Soc., 27, p. 191 Süd-Ost(1901).

Brasilien,

?Amazonas

(var. vel ab.?)

melitia Druce, Biol. Centr.-Amer., Lep. Het., 2, p. Guatemala 104 , t. 51 , f. 3 (1892).

var. o bliquaria Schaus, Tr. Amer. Ent. Venezuela Soc., 27, p. 191 (1901) (Sao Paulo). - bis abruptaria Warr., Proc. U. S. Mus., 30, p. Cayenne, 427 (1906) (Fr. Guiana).

Bolivien, Brasilien

transecta Schaus, Ann. Mag. Nat. Hist., (8) 10, Costa-Rica, p. 295 (1912) (Costa Rica).

Panama

prunelliaria (part.) Druce, Biol. Centr.Amer., Lep. Het., 2, p. 103 (1892) (err. aet.).

dispilota Prout, Nov. Zool., 27, p. 306 (1920) (Fr. Columbien, Guiana). $\quad$ Guayana

decussata Scheller \& Sepp in Sepp, Surin. Vlind t Guayana, 3, p. 287, t. 132 (1855) (Phalaena) (Su- Amazonenrinam). Strom, de line a ta Warr., Proc. U. S. Mus., 30, p. Süd428 (1906) (Fr. Guiana).

Brasilien

(var.) 


\section{Genus Pleuroprucha Saalm.}

Saalm. in Möschl., Abh. Senckenb. Ges., 16, p. 238 (1890) (typus molitaria Möschl.), - Schaus, Tr. Amer. Eint. Soc., 27, p. 193 (1901). - Prout, Nov. Zool., $37: 2$, p. 236 (1932) (emend. pro:) Plauranprucha Möschl. (MS.) teste Saalm., 1. c. (1890).

„Leptomeris Guen." Gmpbg., Nova Acta Acad. Leop. Nat., $49: 4$, p. 333, 34 (1887); $65: 3$, p. 305 (1896) (nec Hib.).

A pa li la c ta Möschl., to e. (1890) (typus pyrrhularia Möschl.).

De pta li a "Hulst, Tr. Amer. Ent. Soc., 23, p. 299 (1896) (typus insularia Hulst = insulsaria Guen.). - Schaus, l.c. (1901).

Il e miptilota Warr., MS. (in Mus. Brit.).

archigetes Prout, Nov. Zool., $37: 2$, p. 236 (1932) Venezuela (Rio Demerara [,Potaro R." ex err.]). bis Fr.-

Guayana, ?ZentralAmerika

protopages Prout, 1. c. (1932) (Maroni R.). Fr.-

Guayana,

Pará

rudimentaria Guen., Spec. Gén. Lép., 9, p. 407 Antillen, (1858) (Ephyra) (Haiti). - Walk., List ZentralLep. Ins., 22, p. 632 (1861) (Eph.). - Butl., Amerika Tr. Ent. Soc. Lond., 1881, p. 333 (1881) bis (Eph.). - Schaus, Tr. Amer. Ent. Soc., 27, Brasilien p. 193 (1901) (D.). - Dyar, Proc. U. S. und Mus., 47, p. 234 (1914) (H.). - Ch. Oberth., Et. Lép. Comp., 12, p. 150 , t. 394, f. 3344 (1916). - Kaye \& Lamont, Cat. Trin. Lep., p. 107 (1927) (Anisodes). - - Prout, 1. c. (1932).

?calidata Walk., List Lep. Ins., 26, p. 1596 (1862) (Acidalia?) (Amazon'). - Butl., t. c., p. 339 (1881) (Acid.). - Druce, Biol. Dentr.-Amer., Lep. Het., 2, p. 120, t. 52, f. 24 (1892) (Acid.).

? dis p un ctata Möschl., Verh. Zool.-Bot. Ges. Wien, 31, p. 409 (1881) (Acidatia) (Paramaribo).

rubescens Dogn., Ann. Soc. Ent. Belg., 50, p. 111 Fr.$(1906)$ (D.).

Guayana

pyrrhularia Möschl., Abh. Senck. Ges., 16, p. 242 Porto Rico (1890) (A.).

molitaria Möschl., t. c., p. 238 (1890) (L.). Porto Rico, ?Dominica

roseipuncta Warr., Nov. Zool., 4, p. 442 (1897) Venezuela, (Surinam). - Schaus, Tr. Amer. Ent. Soc., Surinam, 27, p. 193 (1901) (D.).

?Brasilien

obscurior Schaus, 1. c., (1901)' (D.).

Mexiko 
ochrea Warr., Nov. Zool., 4, p. 442 (1897) (Venezuela).
Venezuela, Br.Guayana, Bolivien, ?Brasilion

atomaria Schaus, t. c., p. 193 (1901) (D.) (Ori- Mexiko zaba) (pr. var.?).

bis

Costa Rica

insulsaria Guen.. Sppec. Gén. Lép., 9, p. 469 (1858) (Acidatia) (Amérique sept.). - Walk., List Lep. Ins., 22, p. 718 (1861) (Acidalia). Pack., Mon. Geom. U. S., p. 335, t. 10, f. 52 (1876) (Acidalia). - Gmpbg., Nova Acta. Acad. Iroop. Nat., $65: 3$, p. 305 (1896) (Leptomeris). - Schaus, 1. c. (1901) (D.). - Hampson, Ann. Mag. Nat. Hist., (7) 7, p. 253 (1901) (Chrysocraspoda insulsatia [err. typogr.]). - Prout, Tr. Ent. Soc. Lond., 1910, p. 215 (1910). - Dyar, Proc. U. S. Mus., 47, p. 234 (1914) (D.) - Ch. Oberth., Et. Lép. Comp., 12, p. 170, t. 399, 7. 3416 (1916) (Acidalia). - Barnes \& Mc Dunnough, Chock List Lep. Bor. Amer., p. 103 (1917). - Großbeck, Bull. Amer. Mus. Nat. Hist, 37, p. 88 (1917). - Ainslie, Ohio Journ. Sci., 23, p. 89 (1923). - Forbes in Leonard, Ins. New York, p. 592 (1928).

placidaria Guen., Spec. Gén. Lép., 9, 469 (1858) (Acidalia) (Amérique sept.). Walk., List Lep. Ins., 22, p. 718 (1861) (Acidalia). - Pack., Mon. Geom. U. S., p. 352 (1876) (Acidalia) - Dyar, List N. Amer. Lep., p. 295 (1902) (Leptomeris). Ch. Oberth., Et. Lép. Comp., 12, p. 170, t. 400 , f. 3417 (1916) (Acidatia). - Barnes \& Mc Dunnough, Check List Lep. Bor. Amer., p. 102 (1917) (Acidalia). - ? Kaye \& Lamont, Cat. Trin. Lep. Het., p. 108 (1927).

invariata Walk., List Iep. Ins., 26, p. 1596 (1862) (Acidalia) (E. Florida). Schaus, 1. c. (1901) (D.).

persimilata Grote, Proc. Ent. Soc. Philad., 1, p. 347, t. 3, f. 5 (1863) (Acidalia) (New York); id., Can. Ent., 3, p. 103 (1871) (Acidalia) (ab.).

insularia (err. transer.) Hulst, Ent. Amer., 3, p. 175 (1887) (Acidatia); id., Fnt. News, 6, p. 71 (1895) (Acidalia); id., Tr. Amor. Ent. Soc., 23, p. 299, 300 (1896) (D.). Dyar, List N. Amer. Lep., p. 291 (1902) (D.).

Biologi e: Pack., l. a., t. 11, f. 32 (1876). Hulst, l. c. (1887). - Ainslio, 1. c. (1923).
Maine bis Texas, Florida, Mexiko bis Panama, Wostind.Inseln, ?Trinidad 
asthenaria Walk., List Lep. Ins., 22, p. 737 (1861) Westindische (Acidalia) (Venezuela). - Schaus, 1. c., Inseln, (1901) (D.). - Prout, l. c. (1910) (insul- Südsaria f.); id., Mitt. Münchn. Ent. Ges., Amerika $21: 1$, p. 18 (1931) (pr. var.?).

i m parat a Walk., op. cit., 26, p. 1598 (1862) (Acidalia) (Jamaica).

in $s$ u l s a ria Kaye \& Lamont, Cat. Trin. Lep. Het., p. 110 (1927) (D.).

tropicaria Schaus, MS. (in coll. E. D. Jones). - truncaria (err. transer.) Edwards, Proc. Ent. Soc. Lond., $2: 3$, p. 58 (1928) (D.) (Brazil).

Biologie: Prout, Can. Ent., 23, p. 369 (1912).

numitoria Druce, Biol. Dentr.-Amer., Lep. Het., Mexiko, 2, p. 121, t. 52, f. 25 (1892) (Acidalia?) Costa-Rica, (Mexico). - Schaus, 1. c. (1901) (D.). Panama

paranaria E. D. Jones, Proc. Zool. Soc. Lond., S.-O.1921, p. 344, t. 3, f. 4 (1921) (Anisodes). Brasilien 14

\section{Genus Bytharia Walk.}

Walk., List Lep. Ins., 31, p. 195 (1864) (typus marginata Walk.).

Longipalpa A. Pagenst., Zoologica, 29, p. 143 (1900) (typus circumalucta A. Pagenst.).

Silva spica Schultze, Philipp. Journ. Sci., $28: 4$, p. 572 (1925) (typus baletensis Schultze).

Morphologie: Schultze, l. c., text-fig. 1 (1925).

uniformis Swinh., Tr. Ent. Soc. Lond., 1902, p. Java, 643 (1902) (Sumatra). - Prout, Nov.Zool., Sumatra, 27, p. 307 (1920); id., Journ. F. iI. S. Borneo, Mus., $17: 1$, p. 55 (1932).

Luzon,

ba letensis Schultze, Philipp. Journ. Sci., Celebes $28: 4$, p. 572 , t. 1 , f. 1 (1925) (S.) (Luzon).

marginata Walk., List Lep. Ins., 31, p. 195 (1864). Ceram

- Swinh., Cat. Lep. Het. Oxf. Mus, 2, p .325 (1900); id., Tr. Ent. Soc. Lond., 1902 , p. $642(1902)$.

var. atrimargo Warr., Nov: Zool., 3, p. Fergusson298 (1896) (Panaethia) (Fergusson I.); p. Insel, 86 (1897) (Xanthomima). - latimargo, Neuguinea Warr., op. cit., 5, p. 10 (1898) (Etna Bay).

lucida Warr., Nov. Zool., 6, p. 14 (1899). - Swinh.' t. c., p. 613 (1902).

Nell-

Hannover

var. (?syn.) circumducta A. Pagenst., NeuZoologica, 29, p. 143 (1900) (L.) (Neu- Jrmmern, pommern). - circumdata Swinh., l. c. (1902). Neu-

Meck\}enburg

var. angusticincta Prout, Nov. Zool., 27, Rendova, 3 p. 306 (1920) (Rendova).

Choiseul 


\section{Genus Prasinochrysa Warr.}

Warr., Nov. Zool., 7, p. 160 (1900) (typus detracta Walk.).

detracla Walk., List Lep. Ins., 31, p. 169 (1864) Columbien, (Chrysauge) (Tenezuela). - Dyar, Proc. U. Panama, S. Nat. Mus., 47, p. 332 (1914) (,Pyrinia“). Tenezuela, fructidora Th.-Nieg, Bull. Soc. Ent. Fr., Brit.1894, p. 109 (1894) (Eurymene) (Pérou). Guayana, cucharis Warr., Nov. Zool., 7, p. 160 Amazonen(1900) (err. det,, nec Drury). Strom,

Peru, Matto-Grosso

quadriplaga $\mathrm{C}$. Felder, Reise Novara, Lep. Het., 2, Fr.2 t. 133, f. 18 (1875) (Numeria) (Guiana). Grayana,

\section{Genus Xanthyris C. Felder}

$X a n t h y r i s$ (Bsd. in litt.) C. Felder, Wien. Ent. Monats., 6, p. 230 (1862) (typus flaveolata L.).

Eugyris Bsd., Lép. Guat., p. 94 (1870) (typus flaveolata L.).

Pachiosia Butl., Ann. Mag. Nat. Hist., (4) 20, p. 128 (1877). - W. F. Kirby, Zool. Rec., 14, p. Ins. 155 (1879) (Pechiosea) (typus flaveolata L.).

supergressa Bastelb., Deutsch. Ent. Zeits., 1909, p. Columbien, 318 (1909) (Columbien). Ecuador

flaveolata L., Syst. Nat. (ed. 10) 1, p. 525 (1758); Costa-Rica (ed. 12) $1: 2$, p. 867 (1767); id., Mus. bis Peru, Lud. Ulr., p. 396 (1764) ("Indiis"). - Cayenne, Cram., Uitl. Kap., $1: 8$, p. 139 , t. 88, f. C Süd-Ost(1776). - Göze, Ent. Beytr., 3:3, p. 321 Brasilien (1781). - F., Spec. Ins., 2, p. 258 (1781); id., Mant. Ins., 2, p. 200 (1787) (flavecolata, exr. typogr.); id., Ent. Syst., $3: 2$, p. 176 (1794). - Stoll, Essai Ord. Syst. Lep., p. 26 (1782). - Gm., Linn. Syst. Nat., 5 : 1, p. 2472 (1790). - [A. Lcht.] Cat. Mus. Zool., p. 127 (1796) (flaveolataria). - Brown, Book Butt., Sphinx. \& Moths, 3, p. 92, t. 32 (1834) (Phal. flaviolata). - Erichs, in Schomburgk, Reis. Br. Guiana, 3, p. 606 (1848) (Euprepia). - O. Feld., Wien. Ent. Monats., 6, p. 230 (1862). - Walk., List Lep. Ins., 35, p. 1878 (1866). - Bsd., Lép. Guat., p. 94 (1870) (Eugyris). - Butl., Ann. Mag. Nat. Hist., (4) 20, p. 128 (1877) (Pachiosia); id., Tr. Ent. Soc. Lond., 1878, p. 61 (1878) (Pachiosia). - Ch. Auriv., K. Sv. Vet.-Ak. Handl. (n. s.) 19 (Recensio Lep. M. L. U.), p. 167 (1882) (Eugyris).

I conograpli ie: Seba, Thesaurus, 4, t. 7 , f. 7,8, t. 13 , f. 3,4 (1765). 
involuta Bastelb., Deutsch. Ent. Zeits., 1909, p. 317 Amazonen(1909) (Pebas).

superba Druce, Ann. Mag. Nat. Hist. 197 (1903) (Flavinia) (Cuzco).

(7) 11, p. Cuzco,

Ia $\mathrm{Paz}$

var. planilimbat a Warr., Nov. Zool., 12, Chanchamayo, 4

p. 316 (1905) (Chanchamayo). Ucayali

\section{Genus Micropos Hb.}

Micropus Hb., Zutr. Exot. Schmett., 1, p. 24 (1818) (indescr.) (nom. praeocc., nec Wolf 1810).

Micropos Hb., Verz. Bek. Schmett., p. 347 (1826) (typus longalis $\mathrm{Hb}$.).

S c a ptia Walk., List Liep. Ins., 2, p. 376 (1854) (typus intercepta TValk.).

A t y ria Warr., Nov. Zool., 2, p. 84 (1895) (err. det., nec H.-Sch!).

S micropus Warr., Nov. Zool., 2, p. 85 (1895) (nom. nov.).

Micropiu us W. F. Kirby in Hb. Zutr. (ed. Wytsman), p. 69 (1912) (nom. nov.).

laeta Walk., List Lep. Ins., 31, p. 179 (1864) Mexiko (Flavinia) (Mexico). - Druce, Biol. Centr.Amer., Lep. Het., 1, p. 150 (1885); 2, p. 406 (1897) (Flavinia). - W. F. Kirby, Syn. Cat. Lep. Het., 1, p. 404 (1892) (Cyllopoda).

pseudis is Bsd., Lep. Guat., p. 93 (1870) (Xanthyris) (Nicaragua). - W. F. Kirby, l. c. (1892) (Cyllopodx).

is is Warr., Nov. Zool., 2, p. 84 (1895), 7 , p. 125 (1900) (Atyria) (err. det., nec Hb.).

var. o $p$ \& Druce, Proc. Zool. Soc. Loud., 1885, p. 529 (1885) (Flavinia). - W. F. Kirby, l. c. (1892) (Cyllopoda). - Warr., Nov. 7 , p. 125 (1900) (Atyria). - laeta var. b Walk., List Lep. Ins., 31, p. 180 (1864) (Flavinia).

bogotensis Dogn., Het. Nouv. Amér. Sud, 13, p. 7 Bogota (1917) (Flavinia) (pr. ab., prob.).

mamillifera Warr., Nov. Zool., 7, p. 125 (1900) West(Atyria?).

bis

Columbien,

Venezuela,

PSurinam

Bogota

intercepla Walk., List Lep. Ins., 2, p. 372 (1854) Mexiko

Ecuador

(Chrysauge (Flavinia)) (Honduras). - bis

Druce, Biol. Centr.-Amer., Lep. Het., 1, p. Nicaragua

151, t. 14 , f. 8 (1885) (Flavinia). - W. F. Kirby, Syn. Cat. Lep. Het., 1, p. 403 (Cyllopoda).

a ntho lia Walk., List Lep. Ins., 2, p. 376 (1854) (Chrysauge (sicaptia); (Nicaragua). - Druce, 1. c. (1885) (Scaptia).

a $d$ u $n$ c a Bsd., Lép. Guat.s p. 93 (1870). (Xanthyris) ("Nicaragua" [Mexico, sec. Druce 1). 
marginata Dogn., Ann. Soc. Ent. Balg., 47, p. 271 Chiriqui

(1903) (S.).

a l cid a mea (part.) Druce, Proc. Zool. Soc.

Lond., 1890, p. 498 (1890); id., Biol. Centr.-

Amer., Iep. Het., 1, p. 407 (1890) (Flavinia) (err. det.).

ochra Druce, Ann. Mag. Nat. Hist., (7) 3, p. 297 Venezuela (1899) (Micropus). - Warr., Nov. Zool., 8, p. 438 (1901) (Cyllopoda).

longicorpus Warr., 1. c. (1901) (Cyllopoda).

Iongalis Hb., Zutr. Exot. Schmett., 1, p. 24, f. Guayana, 131, 132 (1S18) (Microputs) (Brasilien); id., Pará Ver7. Bek. Schmett., p. 317 (1826)! - Walk., List Lep. Ins., 2, p. 377 (1854) (Chrysauge (Micropus)). - H.-Ssh., Samml. AuBereur. Schmett., 1, p. 20 (1856) (Atyria). - Warr., Nov. Zool., 2, p. 85 (1895) (S.). - W. F. Tirby in $\mathrm{Hb}$., Zutr. (ed. Wytsman), p. 69, t. 514 (23), f. 131, 132 (1912) (Micropinus). ab. latifas ciata Warr., 1. c. (1895) (S.).

var: angusta Warr., l. c. (1895) (S.). - Columbien augusta (err. typogr.) Dogn., Ann. Soc. Ent. Belg., 47, p. 271 (1903) (S.).

elegans Druce, Proc. Zosl. Soc. Lond., 1885, p. 529, Feuador, t. 32, f. 20 (1885) (Micropus) (Ecuador). - Columbien, Dogn., Lép. Loja, 2, p. 39 (1891) (Mi- Venezuela cropus).

simplex C. Feld., Reise Novara, Lep. Het., 2, t. Bogota, 105, f. 11 (1874) (Atyria). - ? Möschl., ?Surinam Verh. Zool.-Bot. Ges. Wien, 27, p. 659 (1877) (Atyria). - Warr., Nov. Zool., 2, p. 85

9 (1895) (S.).

\section{Genus Paratyria Warr.}

Warr., Nov. Zool., 2, p. 85 (1895).

darna Sehaus, Proc. Zool. Soc. Lond., 1892, p. 286 Süd-Ost(1892) (Flavinia); id., Amer. Lep., 1, p. Brasilien 17, t. 2, f. 25 (1892) (Cyllopoda). - Warr., 1. c. $(1895)$.

\section{Genus Cyllopoda Dalm.}

Dalm., Anal. Ent., p. 102 '(1823) (typus claudicula Dalm.). Walk., List Lep. Ins., 7, p. 1778 (1856). - Warr., Nov. Zool., 2; p. 84, 85 (1895). - Prout, Ann. Mag. Nat. Hist. (8) 6, p. 239, 240 (1910); id., Nov. Zool., 24, p. 391 (1917).

Flavinia (Chrysauge group 2) Walk., List Iep. Ins., 2, p. 369 (1854) (typus postica Walk.). - Druce, Biol. Centr.Amer., Lep. Het., 1, p. 150 (1885). 
claudicula Dalm., Anal. Ent., p. 102 (1823). - Brasilien Walk., List Lep. Ins., 7, p. 1778 (1856). W. F. Kirby, Cat. Isep. Het., 1, p. 403, (1892). - Prout, Nov. Zool., 23, p. 173 $(1916)$.

dichroa Perty, Del. Anim. Artic. Bras. (3), p. 161 , t. 32 , f. 8 (1833) (Callimorpha). Walk., List Lep. Ins., 2, p. 371 (1854) (Chrysauge (Flavinia)). - W. F. Kirby, l. c. (1892). - Strand, Archiv f. Naturg., 1920, A. T, p. 136 (Flavinia). - Prout, t. c., p. $174(1916)$.

angusta Warr., Nov. Zool., 4, p. 419 (1897).

Bolivien

jatropharia L., Syst. Nat. (ed. 10), 1, p. 523 (1758) (ed. 12) $1: 2$, p. 865 (1767); id., Mus. Lud. Ulr., p. 395 (1764) (America). - Olerek, Ic. Ins. Rar., 2, t. 55, f. [3] (1764). - F., Syst. Ent., p. 627 (1775); id., Spec. Ins., 2, p. 250 (1781); id., Mant. Ins., 2 , p. 193 (1787); id., Ent. Syst., 3:2, p. 154 (1794). - Göze, Ent. Beytr., 3:3, p. 214 (1781). - Gm., Linn. Syst. Wint., 1:5, p. 2469 (1790). - Ch. Auriv., K. Sv. Vet.Ak. Handl., (n. 8.) 19 (Recensio Lep. M. L. U.) p. 166 (1882) (Atyria). - W. F. Kirby, Cat. Lep. Het., 1, p. 403 (1892). Dogn., Ann. Soc. Ent. Belg., 44, p. 214 (1900) (Atyriodes). - Prout, Entom., 41, p. 78 (1908). - Kaye \& Lamont, Cat. Trin. Lep. Het., p. 110 (1927).

jatrophae Hb., Zutr. Exot. Schmett., 2, p. 31 (1822) (Atyria).

o siris Druce, Biol. Centr.-Amer., Lep. Het., 1, p. 150 (1885) (F.) ((,Costa Rica“" [err. loc.?]).

var. $p$ u $t a$ Strand, Archiv f. Naturg., $86 \mathrm{~A}: 7$, p. 137 (1921) (Trinidad) $0^{7}$; id., Entom. Nachrichtsbl., 1, p. 24 (1927) (? ab. loc.).

var. osiris Oram., Uitl. Kap., 2 : 10, p. 28, t. $115 \mathrm{E}$ (1777) (Surinam). - Stoll, Essai Ord. Syst. Lép., p. 26 (1782), - Guér. in Tabl. Encycl. et Méth., 18, p. 25, t. 88, f. 7 (1818) (Phalaena). - Verloren, Cat. Lop. Crameri, p. 53 (1837) (Callimorpha). Walk., List Lep. Ins., 2, p. 370 (1854) (Chrysauge (Flavinia)). - H.-Sch., Samml. AuBereur. Schmett., 1, p. 20 ((1856) (Atyria). - ? Möschl., Verh. Zool.-Bot. Ges. Wien, 27, p. 659 (1877) (Atyria). - Prout, Entom., 41, p. 78 (1908); id., Tr. Ent. Soc. Lond., 1910, p. 230 (1910). - jatropharia Cram., t. c., p. 151 (1777). - osirisaria A. Lcht., Cat. Mus. Zool., p. 127 (1797). latimargo Warr., Nov. Zool., 4, p. 420 (1897) (Rio Demerara). - (s. nom.) Merian, Ins. Surin., t. 72, f. [1] (1705) (icon., s. text.).

Ostl. Br.- 
var. o vata TVarr., Nov. Zool., 14, p. 198 (1907) (Carabaya). - jatropharia Missn. in Stübel, Reisen, p. 75 (1890) (Flavinia). postica Druce, Biol. Centr.-Amor., Lep. Het., 1, p. 115 (18S5) (Flavinia) (err. det., nec Walk.). - pseudisis Warr. olim., in Mus. Brit. (err. det., nec Bsd.).

postica Walk., List Ẽep. 'Tns., 2, p. 371 (1854) (Chrysauge (Flavinia)). - ? Dogn., Lép. Loja, 2, p. 39 (1891) (Flavinia). - W. F. Kirby, Cat. Lep. Het., 1, p. 404 (1892). Prout, Nov. Zool., 23, p. 174 (1916) (Atyria). gibbifrons Prout, Nov. Zool., 24, p. 391 (1917).

nigrivena Prout, t. c., p. 392 (1917).

latiflava Warr., Nov. Zool., 12, p. 312 (1905) (Colombia).

expansifascia Prout, Nov. Zool., 24, p. 391 (1917) (Charaplaya).

breviplaga Dogn., Ann. Soc. Ent. Belg., 50, p. 108 (1906) (Pérou [Nord-Est]).

versicolor Dogn., Ann. Soc. Ent. Belg., 52, p. 17 (1908) (Loja) (ub. vel var.?).

roxana Druce, Proc. Zool. Soc. Lond., 1885, p. 529, Ost-Peru t. 32 , f. 19 (1885) (Flavinia) - W. F. Kirby, Cat. Lep. Het., 1, p. 404 (1892).

angustislriga Warr., Nov. Zool., 11, p. 14 (1904). Süd-OstPeru

bipuncta Warr., Proc. U. S. Mus., 30, p. 409 Peru 12

\section{Genus Afyriodes Warr.}

Warr., Nov. Zool., 2, p. 84 (1895) (typus approximans Warr., err. det., nec Walk.). - Prout, Ann. Mag. Nat. Hist., (8) 6, p. 239 (1910) (Cyllopodic sect.).

parapostica Dogn., Ann. Soc. Ent. Belg., 44, p. 214 Ecuador (1900).

jalapac Schaus, Ent. Amer., 6, p. 45 (1890) (Fla- Mexiko vinia) (Jalapa). - W. F. Kirby, Cat. Lep. bis Het., 1, p. 404 (1892). - Strand, Archiv f. Ecuador Naturg., 1920, A. 7, p. 135 (Flavinia). cyrene Druce, Biol. Centr.-Amer., Lep. Het., 1, p. 406 (1897) (Flavinia) (err. det., nec Druce 1885).
Honduras,

Nicaragua,

icuador

Bolivien

enezuela,

Venezuela, ?Brasilien

Brasilien

Br.-

Guayana,

?Brasilien

Bolivien

Nord-Ost-

Peru,

?Süd-
Ecuador 
albiventris Walk., List Lep. Ins., 7, p. 1657 (1856) Venezuela, (Lyces) (Amazon Valley). - Schaus, Proc. AmazonenZool. Soc. Lond., 1896, p. 636 (1896) (Fla- Strom, vinia).

ab. cren u la t a Warr., Nov. Zool., 4, p. 419 Ost-Peru

(1897) (Rio Ucayali). - vilina Druce, Ann. Mag. Nat. Hist., (9) 3, p. 297 (1899) (Flavinia) (E. Peru).

isthmica Prout, Ann. Mag. Nat. Hist., (8) 6, p. Peru, 239 (1910) (Cyllopoda).

?Columbien

globulata Warr., M.S. (in Mus. Tring)

figulatum Dogn., Ann. Soc. Ent. Belg., 50, p. 107 (1906).

cyrene Druce, Proc. Zool. Soc. Loud., 1885, p. 529 (1885) (Flavinia) (Bolivia). - W. F. Kirby, Cat. Lep. Het., 1, p. 404 (1892) (Cyllopodx). - Prout, Ann. Mag. Nat. Hist., (8) 6, ј. 439 (1910) (C. (A.)).

ab. coro $i$ c a $n$ is Strand, Archiv f. Naturg., Bolivien, 86 A : 7, p. 138 (1921) (Cyllopodu) of (Bo- Peru livien); id., Entom. Nachrichtsbl., 1, p. 24 o' (1927) (Cyllopoda).

pachiteac Strand, Archiv f. Naturg., 86 A. 7, p. Peru: $139 \sigma^{\top}$ (1921) (Cyllopoda).

Pachitea

jancira Schaus, Proc. Zool., Soc. Irond., 1892, p. 286 Rio do (1892) (Flavinia); id., Amer. Lep., p. 17, Janeiro t. 2, f. 20 (1892) (Cyllopoda). - Dogn., Ann. Soc. Ent. Belg., 44, p. 214 (1900). Strand, Archiv f. Naturg., 1920, A. 7, p. 139 (Cyllopoda).

var. lugens Prout, Nov. Zool., 27, p. 311 Parana (1920) (Cyllopola). - approximans Warr., (Süd-OstNov. Zool., 2, p. 84 (1895) (err. det., nec Brasilien) 8 Walk.)

\section{Genus Afyria H.-Sch.}

Hb., Ztr. Exot. Schmett., 2, p. 31, 1822 (indescr.). - H.-Scl.; Samml. Außereur. Schmett., 1, p. 19 (1856) (typus isis Hb.). Burm., Descr Rép. Arg., 5 (Lép. 1) f. 419 (1878). - Prout, Tr. Ent. Soc. Lond., 1910, p. 229 (1910).

Fla vinia (part.) Walk., List Lep. Ins., 2, p. 369 (1854). Druce, Biol. Centr. Amer., Lep. Het., 1, p. 150 (1885) (nec sect. typ.).

Phalcidona (part.) Walk., List Lep. Ins., 31, p. 135 (1861) (err. pro Phalcidon. Walk. 1854) (nec. sect. typ.). - Druce, Biol. Centr-Amer., Lep. Het., 1, p. 145 (1885).

mnemosyne Prout, Nov. Zool., 23, p. 174 (1916). Peru

aleidamea Druce, Proc. Zool. Soc. Lond., 1890, Ecuador, p .498 (1890) (F.) (Ecuado1 ). - W. F. Peru Kirby, Cat. Lep. Het., 1, p. 402 (1892) (Cyllopoda). 
allogaster Prout, Ann. Mag. Nat. Hist., (9) 2, p. Peru 415 (1918).

limbata Butl., Ann. Mag. Nat. Hist., (4) 12, p. 228 Peru, (1873) (Chrysauge $(F)$.$) (Tuasampilla, Bolivien$ Peru).

eireumdata Missn. in Stübel, Reisen, p. 101, 130, t. Peru, 4, f. 22 (1890) (Chrysauge) (Rio Pongo) Bolivien, - W. F. Kirby, Cat. Tep. Het., 1, p. 404 ?Ecuador, (1892) (Cyllopoda). - Dogn., Lép. Loja, 3, ?Argentinien p. $72(1894)$ (F.) (pr. f. vel syn.?). (var.?)

centralis Dogn., Mém. Soc. Ent. Belg., 18, p. 158 Peru (1911) (Cyllopada).

Iemonia Druce, Proc. Zrool. Soc. Lond., 1890, p. Ecuador 499 (1890) (F.).

eompensata Dogn., Ann. Soc. Ent. Belg., 50, p. Peru 108 (1906) (Hillapani) (Cyllopoda).

albifrons Prout, Nov. Zool., 23, p. 174 (1916). Peru

quicha Schaus, Proc. Zool. Soc. Lond., 1892, p. 286 Peru (1892) (F.); id., Amer. Lep., p. 18, t. 2, f. 23 (1892) (Cyllopoda). - Strand, Archiv f. Naturg., 1920, A. 7, p. 137 (Flavinia).

isis Hb., Zutr. Exot. Schmett., 2, p. 31, f. 385, Columbien, 386 (1822) (Brasilien). - M.-Sch., Samml. Paraguay, Außereur. Schmett., 1, p. 20 (1856). - Brasilien Burm., Descr, Rép. Arg., 5 (Lép. 1), p. 420 (1878). - W. F. Kirby, Cat. Lep. Het., 1, p. 403 (1892) (Cyllopoda). - Prout, Ann. Mag. Nat. Hist., (9) 1, p. 31 (1918) (F.).

app roximans Walk., List Lep. Ins., 2, p. 370 (1851) (Chrysange) (s. loc.).

ab. b o e ta Mabilde, Guia Pract. Coll. Ins., p. 229 , t. 24, f. 7 (1896) (Atyrias) (err. transar. et det., nec laeta Walk.).

ab. alegrensis Strand, Archiv f. Naturg., $86 \mathrm{~A}: 7$, p. 137 (1921) (quicha var.) (Porto Alogre); id., Fnt. Nuchrichtsbl., 1, p. 24 (1927) (Flavinia).

dubia Schaus, Proc. Zool. Soc. Tond., 1892, p. Brasilien 286 (1892) (F.); id., Aner. Lep., p. 17, t. 2, f. 25 (1892) (Cyllopoda) (pr. f.?).

is is Prout, Tr. Ent. Soc. Lond., 1910, p. 230 (1910).

stenoehora Prout, Ann. Mag. Nat. Hist., (9) 1, p. Brasilien

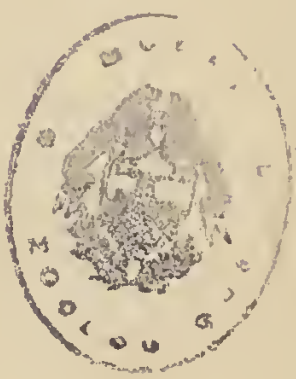
31 (1918) (F.).

approximans (part.) Walk., 1. c. (1854) (F.) (nee typ.).

ehibeha Schaus, Pros. Zool. Soc. Lond., 1892, p. 287 Peru bis (1892) (F.) (Peru); id., Amer. Lep., p. 18, Argentinien, t. 2, f. 21 (1892) (Cyllopoda). - Prout, Tr. Brasilien Ent. Soc. Lond., 1910, p. 229 (1910) (A.); Mitt. Münch. Ent. Ges., $21: 1$, p. 17 (1931) $(F$.$) .$

o siris Burm., Desır. Rép. Arg., 5 (Lép. 1), p. 420 (1878) (err. det, nec Oram.).

ab. tenu is Warr., Nov. Zool., 12, p. 312 (1905) (Cyllopoda) (S. E. Brazil). 
basina Bsd., Lép. Guat., p. 93 (1870) (Xanthyris) Peru bis (Guatemala, err. loc.; Venezuela, err. loc.?). Argentinien, - Druce, Biol. Centr.-Amer., Lep. Het., 1, Brasilien p. 151 (1885) (F.). - W. F. Kirby, Cat. Lep. Het., 1, p. 403 (1892) (Cyllopoda). Prout, Mitt. Münchn. Ent. Ges., $21: 1$, p. 18 (1931) (F.).

cast in a Bsd., 1. c. (1870) (Xanthyris) (Santa Catharina).

semidivisa. Warr., Nov. Zool., 4, p. 420 (1897) (Cyllopoda) (Entre Rios). - Prout, Tr. Ent. Soc. Lond, 1910, p. 230 (1910).

e ion Druce, Ann. Mag. Nat. Hist., (7) 3, p. 296 (1899) (F.) (Bolivia).

cuneifera Warr., Nov. Zool., 11, p. 15 (1904) (Cyllopoda chibcha ab.) (Paraguay).

gracillima Warr., Nov. Zool., 14, p. 197 (1907) Paraguay (Cyllopoda).

Durnfordi Druce, Ann. Mag. Nat. Hist., (7) 3, Paraguay, p. 296 (1899) ( $F$.) (Buenos Aires). . Brasilien,

ab. (?) attenu ata Warr., Nov. Zool., 7, Argentinien p. 135 (1900) (Cyllopoda) (Sao Paulo).

quadriradiata Weym., Berl. Ent. Zeits., 46, p. 303, Westt. 5, f. 15 (1901) (Cyllopoda). Ecuador

subdichroa Dogn:, Ann. Soc. Ent. Belg., 44, o. 214 Ecuador (1900).

dichroides Prout, Nov. Zool., 23, p. 173 (1916) (S. Peru, Brazil) (pr. f.?).

Bolivien, Brasilien

radiata Warr., Proc. U. S. Mus., 30, p. 410 (1906) Süd-Ost(Cyllopoda) (huj. gen.?).

Brasilien

volumnia Druce, Ann. Mag. Nat. Hist., (7) 3, p. Ost-Peru $296(1899)(F)$.

cruciala Warr., Proc. UT. S. Nat. Mus., 30, p. 404 Bolivien 71906) (sequ. var. vei a.b.?).

f. mat utine ll a Strand, Archiv f. Naturg., Mapiri $86 \mathrm{~A}: 7$, p. 136 (1921) (F.); id., Entom. (Bolivien) Nachrichtsbl., 1, p. 24 (1927).

matutina Walk., List Lep. Ins., 31, p. 135 (1864) Peru (Phalcidona). - W. F. Kirby, Cat. Lep. Het., 1, p. 403 (1892) (Cyllopoda). - Strand, Archiv f. Naturg., 86, A. 7, p. 136 (1921) (Flavinia).

ab. malanciata Strand, 1. c. (1921) (F.); Malankiata id., Entom. Nachrichtsbl., 1, p. 24 (1927). (Poru)

nanipennis Warr., Nov. Zool., 7, p. 125 (1900): (Cyl- Ostlopoda).

Ecuador

vespertina Walk., List Lep. Ins., 31, p. 135 (1864) Columbien, (Phaleidona) (Bogota). - Druce, Biol. Amazonas Centr.-Amer., Lep. Het., 1, p. 145 (1885) (Phalcidona). - W. F. Kirby, Cat. Lep. Het., 1, p. 403 (1892); id., Handb. Lep.; 3 , p. 187, t. 89 , f. 2 (1897) (Cyllopoda). - 
Warr., Nov. Zool., 4, p. 420 (1897) (Cyllopoda).

o se ra Bsd., Lép. Guat., p. 93 (1870) (Xanthyris) (Honduras \& Mexique, ex err.).

ab. reducta C. Felder, Reise Novara, Lep. Het., t. 105, f. 12 (1874) (Bogota).

velata Druce, Biol. Centr-Amer., Lep. Het., 1, p. Panama,

151 , t. 14 , f. 9 (1885) (F.) (Chiriqui). - ?Costa-Rica

W. F. Kirby, Cat. Lep. Het,, 1, p. 404 27

(1892) (Cyllopoda).

\section{Genus Formiana Druce}

Druce, Proc. Zool. Soc. Lond., 1885, p. 528 (1885).

maenades Druce, l. c., t. 32 , f. 18 (1885) (Bolivia). Peru,

meander W. F. Kirby, Cat. Lep. Het., 1,

Bolivien 1 p. 404 (1892) (err. transer.).

\section{Genus Oncopus H,-Sch.}

H.-Sch., Samml. Außereur. Schmett., 1, p. 19 (1856) (typus citrosa Hb.-Gey.).

Neochorista Warr., Nov. Zool., 4, p. 440 (1897) (typus transpecta $\mathrm{Hb}$-Gey).

transpecta Hb.-Gey., Zutr. Exot. Schmett., 4, „1. 8 Columbien, f. 605,606 (1832) (Paida) (,St. Thomas" - Walk., List Lep. Ins., 2, p. 513 (1854) (Paidia). - H.-Sch., Samml. Außereur. Schmett., 1, p. 20 (1856) (transepta, err. transcr.). - W. F. Kirby, Cat. Lep. Het., p. 363 (1892); Itb. Zutr. (ed. Wytsman) p. 36 (1912) (Euphanessa). - Warr., Nov. Zool., 4, p. 440 (1897) 7 .).

citrosa Hb.-Gey., Zutr. Exot. Schmett., 4, p. 18, Brasilien i. 661, 662 (1832) (Paidia) ("Java" [err. loc.]). - Walk., 'List Lop. Ins., 2, p. 513 (1854) (Paidia?). - H.-Sch., Samml. Außereur. Schmett., 1, p. 20 (1856). - TV. F. ñirby, Cat. Lep. Het., p. 362 (1892); id., in H.b. Zutr. (ed. Wytsman) p. 36 (1912) (Pa2 gara) (pr. ab.?).

\section{Genus Myrice Walk.}

Walk., List Lep. Ins., 2, p. 555 (1854) (typus transiens Walk.). Warr., Nov. Zool., 12, p. 315 (1905).

Asiona. Walk., t. c., p. 5577 (1854) (typus inaequalis Walk.):

He migymnodes Warr., Nov. Zool., 1, p. 377 (1894) (typus nitida Warr.); 11, p. 17 (1904). 
nilida Warr., Nov. Zool., 1, p. 377 (1894) (Hemi- Costa-Rica, gymnodes).

?Panama

transiens Walk., List Lep. Ins., 2, p. 555 (1854) Taboga(Venezuela). Insel,

? inaequalis Druce, Biol. Centr. Amer., Trinidad, Lep. Het., 2, p. 409 (1897) (Asiona) (err. Venezuela, det.?).'

Amazon, Matto-Grosso

Steinbachí Prout, Nov. Zool., 37 : 2, p. 236 (1932). Bolivien

inaequalis Walk., List Lep. Ins., 2, p. 557 (1854) Amazon, (Asiona).

Peru

(var.?)

cinctata Dogn., Le Nat., 13, p. 126 (1891) 우 Loja (Eudule) (huj. gen.?).

on ne or in a Dogn., Ann. Soc. Ent. Belg., 38, 5 p. 240 (1894) (Eudule).

\section{Genus Epicosymbia Warr.}

Warr., Nov. Zool., 4, p. 54 (1897) (typus dentisignata Walk.). Prout, Nov. Zool.j 22, p. 324 (1915); id. in Seitz, Macrolep., p. 16, p. 56 (1933).

An a cosymbia Prout, Ann. Trans. Mus., 3, p. 96 (1913); id., Nov. Zool., 22, p. 324 (1915) (typus perśstrigulata Prout). - Epicosymbia sect. B Prout in Seitz, Macrolep., 16, p. 56 (1933).

dentisignata Walk., List Lep. Ins., 26, p. 1605 Transvaal, (1862) (Acidalia ?) (Natal). - Prout, Ann. Natal Transv. MLus., 3, p. 197 (1913); id. in Seitz, Macrolep., 16, p. 56 (1933) (denticulata [err. typog1:] et dentisignata). - Janse, Check List S. Afr. Lep. Het., p. 95 (1917).

perrufa Warr., Nov. Zool., 4, p. 54 (1897) (Natal).

perstrigulata Prout, Ann. Trunsv. Mus., 3, p. 197, Transvaal, t. 12 , f. 10 (1913) (A.); (Transvaal); id. Südin Seitz, Macrolep., 16, p. 56, t. $6 \mathrm{~d}$ (1933). Rhodesia - Janse, 1. c. (1917) (A.).

chrysoparalias Prout, Nov. Zool., 24, p. 432 (1917) Goldküste, (Anacosymbic); id. in Seitz, Macrolep., 16, ? Elfenbeinp. 56, t. 6 e (1933) (pr. var.?).

küste,

? Sudan

albivertex Swinl1., Tr. Ent. Soc. Lond., 1892, p. 15 Nord-Indien (1892) (Idaca); id., 1. c., 1894, p. 179 (1894) (Dithalma) (Khasia); id., Cat. Lepp. Het. Oxf. Mus., 2, p. 372 (1900) (Erythrolophus). - Hampson, Faun. Ind., Moths, 3, p. 455 (1895) (Erythrolophus). - Prout, Ann. Transv. Mus., 3, p. 197 (1913) (A.). 
vax. a n c ill la ria Warr., Nov. Zool., 2, p. 99 Süd-Indien (1895) (Semaeopus) (S. America, err. loc.). conspersa Warr., Nov. Zool., 7, p. 107 (1900) Sumba, (Sumba) (pr. var.?).

Timor

spectrum Prout, Nor. Zool., 30, p. 192 (1923); id. Uganda in Seitz, Macrolep., 16, p. 56, t. 5 i (1933).

nitidala Tarr., Nov. Zool., 12, p. 387 (1905) (Co- Transvaal, symbia?) (Natal). - Janse, Check List S. Natal Afr. Lop. Het., p. 95 (1917) (Anacosymbia). - Prout in Seitz, Macrolep., 16, p. 56 (1933).

ab. sub fasciata Prout, 1. c. (1933). albivertex Warr., Nov. Zool., 7, p. 93 (1900). 7 (Isoplenia) (nom. praeocc.) (Natal).

\section{Genus Isoplenia Warr.}

Warr., Nov. Zool., 4, p. 56 (1897). - Prout, Nov. Zool., 22, „p. 324 (1915); id. in Seitz, Macrolep., 16, p. 56 (1933).

trisinuala Warr., Nov. Zool., 4, p. 56 (1897) (Natal). Goldküste, - Hampson, Proc. Zool. Soc. Lond., 1910, Nigeria, p. 476 (1910) (Erythrolopha). - Janse, Angola, Check List S. Afr. Lep. Het., p. 95 (1917). Nyasa, - Prout in Seitz, Macrolep., 16, p. 56, t. 5 e Delagoa, 1 (1933).

Kapland

\section{Genus Isoplenodia Prout}

Prout, Nov. Zool., $37: 2$, p. 237 (1932); id. in Seitz, Macrolep., 16 , p. 56 (1933)。

arrogans Prout, Nov. Zool., $37: 2$, p. 237 (1932); Madagaskar id. in Seitz, Macrolep., 16, p. 57, t. $6 \mathrm{e}$ (1933).

ab. de gener Prout, Nov. Zool., 37 :, p. 237

(1932); id. in Seitz, Macrolep., 16, p. 57

1 (1933) (? sp. div.).

\section{Genus Lissoblemma Warr.}

Warr., Nov. Zool., 9, p. 500 (1902). - Prout, Nov. Zool., 22, p. 324 (1915); id. in Seitz, Macrolep., 16, p. 57 (1933 (typus viridifusi Warr.).

hamularia Snell., Tijd. Ent., 15, p. 79, t. 6, f. f. 11 Sierra-Loone, (1872) (Decetia) (Neder-Guinea). - Prout Nigeria in Seitz, Macrolep., 16, p. 57, t. $6 \mathrm{f}$ (1933). bis viridifusa Warr., Nov. Zool., 9, p. 500 Angols (1902) (Upper Congo). 
Iunuliferata Walk., List Lep. Ins., 26, p. 1522 Süd-Indien (1862) (Decetia) (Canara). - Hampson, $n$ ll. Het., 8, p. 9, t. 142 , f. 1,5 (1891) (Somatina); id., Faun. Ind. Moths, 3, p. 466 (1895) (Somatina). - Warr., Nov. Zool., 9, 2 p. 500 (1902).

\section{Genus Anfanepsia Turn.}

Turn., Proc. Linn. Soc. N. Sth. Wales, $32: 4$, p. 673 (1908).

poliodesma Turn., 1. c. (1908).

Queensland 1

\section{Genus Difhalama Meyr}

Meyr., Proc. Liun. Soc. N. Sth. Wales, (2) 2, p. 835, 840 (1888) (typus cosmospila Meyr.). - Prout, Nov. Zool., 22, p. 324 (1915).

S o matima Turn., Proc. Linn. Soc. N. Sth. Wales, $32: 4$, p. 672 (1908) (err. det., nec Guen.).

punctilinea Swinh., Ann. Mag. Nat. Hist., (7) 9, Westp. 167 (1902, Mar.) (Sterrha).

Australien

tetrasticha Lower, Tr. Roy. Soc. S. Austral., 26, p. 250 (1902, Dec.) (Leptomeris). - Turn., Proc. Linn. Soc. N. Sth. Wales, 32:4, p. 666 (1908) (Loptomeris).

cosmospila Meyr., Proc. Linn. Soc. N. Sth. Wales, Neusüdwales, (2) 2, p. 840 (1888) (Now South Wales). Victoria - Lowor, Vict. Nat., 10, p. 116 (1893). Turn., Proc. Linn. Soc. N. Sth. Wales, 32 : 4, p. 672 (1908) (Somatina).

persalsa Warr., Nov. Zool., 9, p. 116 (1902, Jul.) West(Cinglis). - Turn., Proc. Linn. Soc. N. Sth. Australien Wales, $32: 4$, p. 693 (1908) (Cinglis persalea, err. typogr.).

io paria Swinh., Tr. Ent. Soc. Lond,, 1902, p. 659 (1902, Nov.) (Sterrha).

desueta Warr., Nov. Zool., 9, p. 358 (1902) (Ly- West4 cauges?).

Australien

\section{Genus Trichosferrha Warr.}

Warr., Nov. Zool., 11, p. 515 (1904) (typus olivata Warr.).

olivata Warr., 1. c. (1901) (Carabaya). Ost-

Ecuador,

Ost-Peru

apiozona Prout, Ann. Mag. Nat. Hist., (9), 1, p. 26 Brasilien 2 (1918) (Hamalia). 


\section{Genus Xysirofa Hulst}

Hulst, Tr. Amer. Ent. Soc., 23, p. 301 (1896). - Prout, Nov. Zool., 25 , p. 88 (1918).

rubromarginaria Pack., Proc. Bost. Soc. Nat. Hist., Vereinigte 13, p. 391 (1871) (Acidalia) (California). - Staaten,

Barnes \& Mc Dunnough, Check List Lep. Südl.

Bor. Amer. p. 101 ((1917) (hepaticaria Brit.-

form.). - Barnes \& Benjamin, Pan-Pacif. Columbien

Ent., 4 : 2, p. 89 (1927).

ferruminaria Zill., Verh. Zool.-Bot. Ges.

Wien, 22, p. 478 (1872) (Acidalia) Texas).

- Pack., Mon. Geom., U. S., p. 360 (1876)

(Acidalia). - Gmpbg., Nova Acta Acad.

Leop. Nat., $54: 5$, p. 543 (1890) (Cleta).

rubromarginuta Pack., Mon. Geom. U.

S., p. 339 , t. 10, f. 57 (1876) (Acidalia).

- Gmpbg., t. c., p. 507 (1890) (Arrhostia).

var. (?) vol ucrata Hulst, Ent. Amer., 2, p. '185 (1887) (Acidalia) (Missouri). Grossbeck, Tr. Amer. Ent. Soc., 33, p. 341 (1907) (Eois). - Barnes \& Benjanin, l. c. (1927). - hepaticaria Hulst, Ent. Amer., 2, p. 222 (1887), 4, p. 49 (1888) (Acidalia); id., I'r. Amer. Ent. Soc., 23, p. 301 (1896). Dyar, List N. Amer. Lep., p. 293 (1902); id., Proc. U. S. Mus., 27, p. 901 (1904). Großbeck, Tr. Amer. Ent. Soc., 33, p. 341 (1907) (Xytrota) (err. dot., nec Guen.).

? var. erythrata Hulst, Bull. Brookl. Soc., 3, p. 42 (1880) (Cidaria); id., Ent. Amer., 4, p. 49 (1888) (Acidalia).

\section{Genus Acratodes Guen.}

Guen., Spec, Gén. Lép., 10, p. 57 (1858) (typus phakellurata Guen.).

Le ptostales Möschl., Abh. Senck. Ges., 16, p. 238 (1890) (typus oblinataria Möschl.).

So matina (part.) Guen., t. c., p. 10 (1858). - Prout, Nov. Zool., 25, p. 88 (1918) (nec sect. typ.).

noctuata Guen., t. c., p. 11 (1858). - - Ch. Oberth., Haiti Et. Lép. Comp., 20, p. 217, t. 549, f. 4843 (1923) (S.).

virgota Schaus, Tr. Amer. Ent. Soc., 27, p. 256 Jamaika (19011) (Haemalea).

phakellurata Guen., Spec. Gén. Lép., 10, p. 57 Haiti (1858).

intamiataria Möschl., Abh. Senck. Ges., 16, p. 241 Porto-Rico (1890) (Syllexis).

suavala (Dyar, Psyche, 9, p. 106, Sept. 1900, in- Florida, descr.) Hulst, Journ. N. Y. Ent. Soc., 8, Bahamap. 217 (Dec. 1900) (Eois) (S. Florida). - Inseln Dyar, List N. Amer. Lep., p. 297 (1902). - 
Barnes \& Mc Dunnough, Contr. Lep. N. Amer., 3 : 3, p. 173 (1916); id., Check List Lep. Bor. Amer., p. 101 (1917) (Xystrota). - Grossbeck, Bull. Amer. Mus. Nat. Hist., 27, p. 92 (1917) (Eois).

fusaria Hampson, Ann. Mag. Nat. Hist., (7) 7, p. 253 (1903) (S.) (Nassau I.) (var.?).

B i o log ie: Dyar, 1. c. (1900). - Grossbeck, 1. c. (1917).

Davisi Grossbeck, Bull. Amer. Mus. Nat. Hist., 27, Florida p. 90 (1917) (Eois). - Barnes \& Mc Dunnough, Contr. Lep. N. Amer., $3: 4$, p. 219 (Eois), p. 266, t. 18, f. 5 (1917) (Xystrota). roseicosta Barnes \& Mo Dunnough, op. cit., 2:3, Süd-Texas p. 123, t. 8 , f. 7,8 (1913) (Xysirota); $3: 3$, p. 173 (1916) ( $X$. suzata race?).

cazeca Druce, Biol. Centr.-Amer., Lep. Het., 2, p. Mexiko 120 , t. 52, f. 21 (189?) (Acidalia).

c o mpensata (part.) Druce, t. c., p. 116 (1892) (Ac.).

oblinataria Möschl., Abh. Senck. Ges., 16, p. 239 Porto-Rico, (1890) (L.).

scintillans Warr., Nov. Zool., 11, p. 37 (1904) (Lipomelia) (Br. Guiana).

Jamaika,

Venezuela, Brit.-

Guayana

praepedilaria Möschl., 1. c. (1890) (L.) (Porto- Bahamarico). Inseln se misignat a Dogn., Ann. Soe. Ent. Belg., bis 50, p. 110 (1906) (Lipomelia) (Venezuela). Grenada, flexifascia Prout, Tr. Ent. Soc. Lond., Veneruela 1910 , p. 222 , t. 48 , f. 21 (1910) (Hamalia) (Buenos Aires) (var.?).

adela Dogn., Lo Nat, 12, p. 18 (1890) (Acidalia) Costa-Rica ('Lamora); id., Lép. Loja, 2 , p. 63, t. 6, bis 1. 20 (1891) (Acidalia). - Prout, 1. c. Bolivien, (1910) (Hamalia); id., Nov. Zool., 25, p. 88. Thinidad, (1918) (Somatina). - Schaus, Ann. Mag. Guayana, Nat. Hist, (8) 9, p. 430 (1912) (Ditha- Brasilien dama). - Kaye \& Lamont, Cat. Trin. Lep. Het., p. 109 (1927) (Dithalama).

delila Schaus, Ann. Mag. Nat. Hist., (8) 9, p. 430 Honduras (1912) (Dithadama, err. transer.) (Costa bis lica). - Dyar, Proc. U. S. Nat. Mus., 47, Panama, p. 232 (1914) (Dithadama).

Fr.-

Guayana

pulida Dogn., Ann. Sac. Ent. Belg., 37, p. 427 (1893) Panama (Ephyra) (Loja); id., Lep. Loja, 3, p. 75 bis (1894) (Ephyra). - Dyar, Proc. U. S. Bolivien Mus., 47, p. 234 (1914) (Haemalea pullida, err. typogr.).

villicostala Warr., Proc. U. S. Mus., 30, p. 460 Trinidad, (1906) (Ptychopoda) (Fr. Guiana). Guayana,

Amazonas,

Peru

griseocostala Warr., Nov. Zool., 11, p. 40 (1904) West(Ptychopoda). 
admirabills Ch. Oberth., Et. Ent., 7, p. 33, t. 2, f. Ecuador, 10, 12 ((1883) (Ephyra) (Peru). - Dogn., Peru, Lép. Loja, 2, p. 42 (1891) (Ephyra). - Bolivien, Schaus, Ann. Mag. Nat. Hist., (8) 9, p. 430 Süd-Ost(1912) (Dithadama). - Prout, Nov. Zool., Brasilien 25, p. 88 (1918) („Lipomelia").

angulata Schaus, t. c., p. 429 (1912) (Dithadama) Br.-

(err. transcr.) (Costa Rica). - Dyar, Proc. Honduras,

U. S. M Lus., 47, p. 232 (1917) (Dithadama). Costa-Rica,

?admirabilis Druce, Biol. Contr.-Amer., lep. Panama,

Het., 2, p. 105 (1892) (Aselloles). Ecuador,

? Mexiko

exaeta Prout, Nov. Zool., 25, p. 88 (1918) (S.). Süd-Ost-

\section{Genus Hamalia $\mathrm{Hb}$.}

Ha malea Hb., Zutr. Exot. Schmett., 2, p. 11 (1822)

(indeser.). - Warr. et al., passim.

Hamalia Hb., Verz. Bek. Schmett., p. 309 (1826). -

Prout, Tr. Ent. Soc. Lond., 1910, p. 222 (part.) (1910)

(typus delotaria Hb.).

ustaria Guen., Spec. Gén. Ióp., 9, p. 484 (1858) Fr.-

(Acidalia). - Walk., List Lep. Ins., 22, p. Guayana

736 (1861) (Acidalia). - Ch. Oberth., Et.

Lép. Comp., 12, p. 172, t. 400 , f. 3433 (1916) (sequ. ab.?).

delotaria Hb., Zutr. Exot. Schmett., 2, p. 11, f. Venezuela, 235, 236 (1822) (Haem.); Verz. Bek. Guayana,

Schmett., p. 309 (1826) (Surinam). - Guon., Amizonas

Spec. Gén. Lép., 9, p. 484 (1858) (Acicklia).

- Walk., List Lep. Ins., 22, p. 736 (1861)

(Acidalia). - W. F. Kirby, in Hb. Zutr. (ed. Wytsman), p. 64, t. 532 (41), f. 235 , 236 (1912) (Idaea).

partita Dogn., Ann. Soc. Ent. Belg., 44, p. 215 Ecuador (1900) (Acidalia) (Ecuador).

de lo ta ria Dogn., Lép. Ioja, 2, p. 42 (1591) (Acidalia). - Druce, Biol. Centr.-Amer., Lep. Het., 2, p. 119 (part.) (1892) (Aci-

3 dalia).

bis

Bolivien

\section{Genus Crypsifyla Warr.}

Warr., Nov. Zool., 7, p. 152 (1900) (typus quinquelineata Dogn.).

imitans Dogn., Ann. Soc. Ent. Belg., 44, p. 216 Costa-Rica, (1900) (Acidalia) (Zamora) (huj. gen.?'). Ecuador

bis

Bolivien

micaceata Walk., List Lep. Ins., 22, p. 736 (1861) Venezuela, (Acidalia) (Venezuela). 
mantaria E. D. Jones, Proc. Zool. Soc. Lond., 1921, Südostp. 345, t. 3, f. 10 (1921) (Haemátia) (pr. Brasilien f.?).

quinquelineata Dogn., Le Nat., 12, p. 10 (1890); Ecuador id., Lép. Loja, 2, p. 63 , t. 6, f. 18 (1891) bis (Acidalia) (Loja) (nec quinquelinearia Pack., Bolivien 1871, - ata Pack., 1876, ex err.). Warr., Nov. Zool., 7, p. $152(1900)$.

confusaria Dogn., MS. (Acidalia) (nom. 3 nov.).

\section{Genus Scelolophia Hulst}

Hulst, Tr. Amer. Ent. Soc., 23, p. 301 (1896) (typus formosa Hulst.). - Dyar, List N. Amer, Lep., p. 294 (1902). - Prout, Tr. Ent. Soe. Lond., 1910, p. 220, 225 (1910); id., Proe. Zool. Soc. Lond., Lond., 1916, p. 147 (1916). - Barnes \& Mc Dunnough, Check List Lep. Bor. Amer., p. 101 (1917).

Calyptocome Warr., Nov. Zool., 7, p. 148 (1900) (typus pannaria Guen.). - Dyar, Ins. Ins. Mens., 1:7, p. 79; $1: 9$, p. 120 (1913).

W a u ch u la Hulst, Journ. N. Y. Ent. Soc., $8: 4$, p. 216 (1901) (typus rubrotincta Hulst).

Iaevitaria Hb.-Gey., Zutr. Exot. Schmett., 5, p. 20, Florida f. 873,874 (1837) (Eois) (Georgia). - bis Texas, Guen., Spec. Gén. Lép., 9, p. 471 (1858) Bermuda, (Acidalia). - Walk., List Lep. Ins., 22, ?Californien p. 719 (1861) (Acidatia). - Hulst, Tr: Amer. Ent. Soc., 23, p. 303 (1896) (Leptomenis). - Warr., Nov. Zool., 2, p. 95 (1895) (Eois). - Dyar, List N. Amer. Lep., p. 296 (1902) (Eois). - F. M. Jones, Ent. News, 21, p. 167 (1910) (Eois). - Barnes \& Mc Dunnough, Oheck List Lep. Bor. Amer., p. (1917). - Grossbeck, Bull. Amer. Mus. Nat. Hist., 37, p. 91 (1917) (Eois) (huj. gen.?).

flo $r i d$ a ta Pack., Mon. Geom. U. S., p. 319, t. 10, f. 36 (Eacacidalia) (Texas). - Gmpbg., Nova Acta Acad. Leop. Nat., $54: 5$, p. 543 (1890) (Cleta).

uniformata Warr., Nov. Zool., 7, p. 151 (1900) Trinidad (Craspedia) (sequ. f.?).

subroseata Guen., Spec. Gén. Lép., 9, p. 482 (1858) Venezuela, (Acidalia) (Cayenne). - Walk., List Lep. Cayenne, Ins., 22, p. 735 (1861) (Acitlatia). - Ch. Amazonas, Oberth., Et. Lép. Comp., 12, p. 171, t. 400, Bolivien, f. 3431 (1916) (Acizlatia). circum duct a Warr., Nov. Zool., 7, p. 153 (1900) (Hacmalea) (Venezuela).

i norn at a Warr., Nov. Zool., 11, p. 33 (1904) (C.) (Venezuela). - ? Dyar, Ins. Ins. Mens., 1, p. 87 (1913) (Cinglis).

penumbrata Warr., Nov. Zool., 7, p. 154 (1900) Dominica (Haemalea). 
Ilamaria Schaus, Pros. U. S. Nat. Mus., 27, p. 193 (1901) (Haemalea).

Süd-Ost-

Brasilien,

Paraguay

roseoliva Warr., Nov. Zool., 7, p. 148 (1900) (C.) St. Vincent, (St. Vincent). - Prout, Tr. Ent. Soc. Lond., Grenada, 1910 , p. 225 (1910). - Dyar, Ins. Ins. ?Dominica, Mens., 1, p. 87 (1913) (C.). ?St. Lucia, PTobago

var. (? syn.) carnearia Dyar, t. c., p. 85 Jamaika (1913) (C.).

Iesmogramma Dyar, 1. c. (1913) (C.) (Fr. Gui- Trinidad, ana).

t u $r$ b a t a (part.) Prout, Tr. Ent. Soc. Lond., Guayana, 1910 , p. 226 (1910) (? Walk. 1862) (pr. Pará var.?).?

rivuiaria Dyar, 1. c. (1913) (C.).

Panama, ?Honduras

dclectabiliaria Möschl., Abh. Sencl. Ges., 16, p. Porto-Rico 236 (1890) (Zonosoma).

variabiiis Dyar, Ins. Ins. Mens., 1, p. 85 ((1913) Cuba (C.) (praec. var. vel syn.?).

Iepaticaria Guen., Spec. Gén. Lép., 9, p. 471 (1858) Maryland (Acidatia). - Walk., List Lep. Ins., 22, p. 719 (1861) (Acidalia). - Pack., Mon. Geom. U. S., p. 352 (1876) (Acidalia) - Ch. Oberth., Et. Lép. Comp., 12, p. 171, t. 400, 1. 3421 (1916) (Acidalia). - Barnes \& Benjamin, Pan-Pacif. Ent,, 4:2, p. 89 (1927).

Crossii Hulst, Can. Ent., 32, p. 105 (1900) (Eois) (Florida). - Dyar, List N. Amer. Lep., p. 297 (1902) (Eois); id., Ins. Ins. Mens., 1, p. 120 (1913) (C.). - F. M. Jones, Ent. News, 21, p. 167 (1910) (Eois). -

crossi Barnes \& Mc Dunnough, Check List Lep. Bor. Amer., p. 101 (1917).

B i ologie: F. M. Jones, 1. c. (1910).

phorearia Guen., Spec. Gén. Lép., 9, p. 470 (1858) (Acidalia) (Haiti). - Walk., List Lep. Ins., 22, p. 726 (1861) (Acid.). - Möschl., Abh. Senck. Ges., 16, p. 236 (1890) (Zonosoma). - Hampson, Ann. Mag. Nat. Hist., (6) 16, p. $332(1895), \quad(7) 7$, p. 253 (1901) (Rhodostrophia). - Prout, Tr. Ent. Soc. Iond., 1910, p. 225 (1910). - Dyar, Ins. Ins. Mens., p. 86 (1913) (C.).

c o n ces s a ta Walk., List Lep. Ins., 22, p. 659 (1861) (Hyria) (Jamaica).

flavicostaria Möschl., Abh. Senck. Ges., $14: 3$, p. 71 (1886) (Anisodes) (Jamaica).

rubrotincta Hulst, Journ. Y. Y. Ent. Soc., $8: 4$, p. Florida 216 (1901) (W.). - Dyar, Ins. Ins. Mens., 1 , p. 84 (1913) (C.) (sequ. ab.?).

Florida,

BermudaInseln

BahamaInseln, Jamaika bis St. Vincent 
purpurascens Hulst, Cam. Ent., 32, p. 105 (1900) (Eois). - Dyar, List N. Amer. Lep., p. 296 (1902) (Eois); id., Ins. Ins. Mens., 1, p. 84 (1913) (C.). - Barnes \& Mu Dunnough, Check List Lep. Bor. Amer., p. 101 (1917).

nycteis Druce, Biol. Centr.-Amer., Lop. Het., 2, p. Mexiko 122 , t. 52, f. 28 (1892) (Acidalia). (Mexico). bis Nordignifer Dyar, Proc. U. S. Nat. Mus., 47, Columbien p. 234 (1914) (C.) (ex err. pro ignifera Warr.)

var. (?ab.) a st o ta Schaus, Tr. Amer. Fnt. Venezuela Soc., 27, p. 256 (1901) (Haemalea).

var. ign ifera Warr., Proc. U. S. Nat. Mus., Guayana 30, p. 436 (1906) (Crypsityla) (Fr. Guiana). - Dyar, Ins. Ins. Mens., 1, p. 84 (1913) (C.).

var. latifasciata Bastelb., Ent. Zeits. WestStuttg., 22, p. 159 (1908) (C.?). Columbien, ? Peru

lltioralls Prout, Nov. Zool., 27, p. 307 (1920). West-Peru

amechana Dyar, Ins. Ins. Mens., 1, p. 84 (1913) Mexiko (C.).

penthemaria Dyar, t. c., p. 81 (1913) (C.) (s. 1.). Cuba

purpurata Warr., Proc. U. S. Nat. Mus., 30, p. Mexiko 438 (1906) (Crypsityla turbata var.). Dyar, t. c., p. $80^{\circ}(1913)$ (C.).

subrubella Warr., t. c., p. 437 (1906) (Crypsityla) Fr.(Maroni R.). - Dyar, t. c., p. 83 (1913) Guayana, (C.) (sequ. var.?).

subrosea Warr., Nov. Zool., 12, p. 321 (1905) (Cryp- Costa-Rica sityla) (Colombia); ? Proc. U. S. Mus., 30, bis Peru, p. 437 (1906) (Crypsityla). - Schaus in Paraguay, coll., see. Dyar, t. c., p. 84 (1913). Süd-Ostconcoloraria E. D. Jones, MS. (err. det., Brasilien nec Dogn.).

ptyctographa Dyar, t. c., p. $83^{\circ}(1913)$ (C.) (Ta- Costa-Rica boga I.).

bis Ecuador, Amazonas, Bolivien

rectimargo Dyar, l. c. (1913) (C.).

Venezuela

hegeter Dyar, 1. c. (1913) (C.) (Costa Rica). Costa-Rica, Fr.Guayana

phryctaria Dyar, t. c., p. 82 (1913) (C.). Mexiko, Ecuador

pappasaria Dyar, 1. c. (1913) (C.) (?pannaria Cuba, subsp.). - ? Kaye \& Lamont, Cat. Trin. ?Trinidad Iep. Het., p. 110 (1924). 
? subroseata H.-Sch., C. B. Zool-Min. Ver. Regensb., 24, p. 181 (1870) (Acidalia) (nom. praeoce, nec Guen. 1858).

olivaceata Warr., Nov. Zool., 8, p. 455 (1901) (Cras- Tenezuela pedia) (? sequ. f.).

pannaria Guen., Spoc. Gén. Lép., 9, p. 470 (1858) Südöstl. (Acidalia) (Amér. sept.). - Walk., List Vereinigte Isp. Ins, 22, p. 7/8 ((1861) (Acid.). - Staaten, Pack., Mon. Geom. U. S. p. 336 , t. 10, f. Bermuda53 (1876) (Acid.). - Hulst, Ent News, 6, Inseln, p. 73 (1895) (Acid.); P id., Tr. Amer. Ent. Jamaika, Soc., 23, p. 302 (1896) '(Leucophthalmia). Mexiko - Warr., Nov. Zool., 7, p. 148 (1900) (C.). bis - J. B. Smith, Rep. Ins. N. Y., 1909, p. 498 (1909). - Prout, T1. Ent. Soc. Lond, 1910 , p. 226 (1910). - Dyar, Ins. Ins. Mens., 1, p. 82 (1913) (C.). - Ch. Oberth., Et. Lép. Comp., 12, p. 170 , t. 400 , f. 3418 (1916) (Acid.). - Barnes \& Mc Dunnough, Check List Lep. Bor. Amer., p. 101 (1917).,

o $r$ e $m$ a la ria Walk., List Lep. Ins., 26, p. 1614 (1862) (Acid.) (s. loc.).

$p u r p u r i s$ ata. Grote, Can. Ent., 3, p. 103 (1871) (Acid.) (Alabama). - Grossbeck, Tr. Amer. Ent. Soc., 33, p. 341 (1907); id., Bull. Amer. Mus. Nat. Hist., 37, p. 89 (1917). F. M. Jones, Ent. News, 21, p. 167 (1910). - Dyar, Ins. Ins. Mens., 1, p. 120 (1913) (C.).

concoloraria Druce, Biol. Centr.-Amer., Lep. Het., 2, p. 121 (1892) (Acid.) (err.' det.).

form s a Hulst, Tr. Amer. Ent. Soc., 23, p. 302 (1896) (Texas). - Dyar, List N. Amer. Lep., p. 294 (1902).

gu st a ria Schaus, MS. (Mexioo).

ab. borrigaria Warr., Proc. U. S. Mus., 30, p. 436 (1906) (Crypsityla) (Mexico).

turbata Walk., Tist Lep. Ins., 26, p. 1755 (1862) (Cambogia) (Brazil). - Warr., Proc. U. S. Mus., 30, p. 437 (part.) (1906) (Crypsityla). - Prout, Tr. Ent. Soc. Lond., 1910, p. 226 (1910). - Dyar, Ins. Ins. Mens., 1, p. 87 (1913) (C.) (pr. var.?).

$p$ u $r$ u $r$ ia Schaus, MS. (S.-O. Brasilien).

terminala (Zell., MS.) Guen., Spoc. Gén. Lép., 9, p. 483 (1858) (Acidalia) (Colombio). Walk., List Lep. Ins., 22, p. 735 (1861) (Acid.). - Hampson, Ann. Mag. Nat. Hist. (6) 16 , p. 332 (1895); id., 1. c., (7) 7, p. 253 (1901) (Somatina). - Dyar, Ins. Ins. Mens., 1 p. 82 (1913) (d.).

rescindaria Walk., List Lep. Ins., 22, p. 742 (1861) (Acid.) (Venezuela).

devolutaria Möschl., Abh. Senck. Ges., 16, p. 239 (1890) (Leptostales) (Portorico). insutaria Möschl., t. c., p. 240 (1890) Columbien, Penezuela, Peru

Argentinien,

Bolivion,

Brasilien

ZentralAmerika, Westindische Inseln, Columbien und Guayana bis Argentinien und SüdBrasilien 
nursica Druce, Biol. Centr-Amer., Lep. Het., 2, p. 119, t. 52, f. 19 (1892) (Acidalia) (Panama).

tragmentata Warr., Nov. Zool., 11, p. 32 (1904) (C.) (Br. Guiana). - Dyar, Ins. Ins. Mens.y 1 , p. 80 (1913) (C.); p. 82 (C. fragmentaria).

ab. exanimaria Dyar, t. c., p. 79 (1913) (C.) (Cuba).

concoloraria Dogn., Io Nat, 12, p. 19 (1890) Costa-Rica (Acidalia); id., Lép. Ioja, 2, p. 63, t. 6, f. bis 19 (1891) (Acidalia) (Loja). - Dyar, Ins. Ecuador, Ins. Mens., 1, p. 81 (1913) (C.). ? Peru

catagompha Dyar, t. c., p. 86 (1913) (C.) (Panama). GorgonaInsel, Panama, ? Fr.Guayana (var.?)

geroeoma Dyar, 1. c. (1913) (C.).

Fr.34

Guayana

\section{Genus Somatina Guen.}

Guen., Spec. Gén. Lép., 10, p. 10 (1858). - Walk., List Lep. Ins., 23, p. 805 (1861). - Moore, Lop. Ceyl., 3, p. 539 (1887). Fampson, Faun. Ind., Moths, 3, p. 463(1895). - Swinh., Tr. Ent. Soc. Lond., 1904, p. 565 (1904). - Prout in Soitz, Macrolep., 4, p. 44 (1913), 16, p. 57 (1933); id., Nov. Zool., 22, 'p. 324, 328 (1915) (typus anthophitata Guen.).

Nebess a Walk., Proc. Nat. Hist. Soc. Glasg., 1 : 2, p. 37 õ (1869) (typus chalyboeata Walk.).

Prasonesis Meyr., Tr. "Ent. Soc. Lond., 1889, p. 486 (1889) (typus microphylla Meyr.).

So matinopsis Warr., Nov. Zool., 3, p. 379 (1896) (typus nigridiscata Warr.).

subviridata Warr., Nov. Zool., 8, p. 10 (1901) Sierra-Leone (Problepsis) (Sierra Leone). - Prout, Nov. bis Zool., 22, p. 328 (1915); id. in Seitz, Ma- Elfenbeincrolep., 16, p .57, t. 5 i (1933). küste

cenlrophora Prout, Nor. Zool., 22, p. 328 (1915) Süd-Afrika (Cape Colony); id. in Seitz, l. c., t. $5 \mathrm{i}$ (1933).

elenophora Prout, 1. c. (1915) (Nairobi); id. in Kenya, Seitz, l. co, t. 6 f (1933). - Janse, Check Transvaal, List S. Afr. Lep. Het., p. 95 (1917). Süd-West-

latonaria Wligr., ofv. K. Sv. Vet.-Ak. Afrika Handl., $32: 1$, p. 119 (1875) (Argyris) (err. det., nec Guon..).

nigridiscata Warr., Nov. Zool., 3, p. 379 (1896) Süd-Java (Somatinopsis).

maeandrata Prout, Nov. Zool., 32, p. 38 (1925) Kedah (zequ. f.?). 
obscuriciliata Wehrłi, Mitt. Münchn. Ent. Ges., Süd-Ost14 , t. 1, f. 2 (1924), 15, p. 49 (1925) China (Lienping).

discata Warr., Nov. Zool., 16, p. 126 (1909). - Hainan Prout, Bull, Hill Mus., 4: 1, p. 430 (1930) (sequ. ab.?).

plynusaria Walk., List Lep. Ins., 26, p. 1581 (1862) Indien, (Anisodes) (Silhet). - Moore, Proc. Zool. Birma, Soc. Lond., 1867, p. 641 (1867) (Anisodes). Hainan, - Cotes \& Swinh., Cat. Moths Ind., pars 4, Formosa p. 585 (1888). - Hampson, Ill. Het., 8, p. 9 (1891); id., Faun. Ind., Moths, 3, p. 464 (1895). - Swinh., Lep. Het. Oxf. Mus., 2, p. 376 (1900). - Prout, Ent. Mitt., 3, p. 240 (1914); id., Bull. Hill Mus., 4:1, p. 131 (1930).

purpurascens Moore, Irep. Ceyl., 3, p. 540, t. 202, f. Ceylon, 2, 2 a (1887) (Ccylon). - Hampson, Ill. Süd-Indien Het., 9, p. 39 (1893) (anthophilata); Faun. Ind., Moths 3, p. 464 (anthophilata f.) (1895) (pr. f.?).

anthophilata Guen., Spec. Gén. Lép., 10, p. 11 Indien, (Somatina); Atlas, t. 18, f. 2 \& explic., p. 3 (Somxtodes) (1858) (Bombay). - Walk., List Lep. Ins., 23, p. 806 (1861). - Swinh., Proc. Zool. Soc. Lond., 1885, p. 859 (1885); Tr. Ent. Soc. Lond., 1890 , p. 191 (1890); id., Ann. Mag. Nat. Hist., (6) 12, p. 151 (1893); id., Cat. Lep. Het. Oxf. Mus., 2, p. 376 (1900). - Warr., Proc. Zool. Soc. Lond., 1888, p. 298 (1888). - Hampson, Ill. Het., 8 , p. 9 (1891); id., Faun. Ind., Moths 3, p. 464 , f. 209 (1895). — ? G. Semp., Reisen Philipp., (2) 6:6, p. 634 (1902). - Tams, Journ. N. H. Soc. Siam, $6: 3$, p. 274 (1924). - J. Joann., Anu. Soc. Ent. Fr., 98, p. 475 (1929). - Prout, Bull. Hill Mus., 4:1, p. 131 (1930):

congruaria Walk., Char. Undescr. Lep., p. $9 S$ (1869) (Anisodes) (N. India) (nom. praeoce., neo Walk. 1862).

ossicolor Warr., Nov. Zool., 5, p. 244 (1898). Sumba

rufifascia Warr., Nov. Zool., 3, p. 379 (1896). - Queenslaud Turn., Pros. Linn. Soc. N. Sth. Wales, $32: 4$, p. 672 (1998).

ab. maculata Warr., Nov. Zool., 5, p. 244 (1898). - Tur.: l. c. (1908).

ab. sordida Warr., l. c. (1898). - Turn., t. c., p. 673 (1908).

ab. $r u b r i d i s c a$ Swinh., Lep. Het. Oxf. Mus., 2, p. 376 (1900). - Turn., t. c., p. 698 (1908).

rosacca Swinh., Tr.. Ent. Soc. Lond., 1894, p. 182 Assam, (1894) (Khasia). - Hampson, Faun. Ind., Penang Moths, 3, p. 465 (1895).

var. $a$ n a e m i c a Prout, Ent. Mitt., 3, p. 240 Formosa (1914). 
microphylla Moyr., Tr. Ent. Soc. Lond., 1889, p. Neuguinea, 486 (1889) (P.) (Br. New Guinea). Nordc a t a c is s a Turn., Proc. Linn. Soc. N. Sth. Queensland Wales, $32: 4$, p. 677 (190S) (Trygodes) (N. Queensland).

irregularis Warr., Nov. Zool,, 5, p. 239 (1898) (Co- Gambia symbia?) (Nigeria); 6, p. 295 (1899) (Li- bis pomelia). - Prout in Seitz, Macrolep., 16, Congo p. 57, t. $6 \mathrm{f}$ (1933) (huj. gen.?).

rlodochila Prout, Nov. Zool., 37:2, p. 237 (1932); Westid. in Seitz, l. c., t. 5 i (1933). Kivu

syneorus Prout, Nov. Zool., 22, p. 326 (1915) (Ga- Sierra-Leone, boon); id. in Seitz, t. c., p. 58, t. $5 \mathrm{k}$ Kamerun, (1933) (sequ. f.?).

Gabun

impunctulata Warr., Nov. Zool., 8, p. 9 (1901) Sierra-Leone (Craspedia). - Prout in Seitz, l. c., t. $5 \mathrm{k}$ (1933).

chalyboeata Walk., Tr. N. H. Soe. Glasg., 1, p. 375 Gambia, (1869) (N.) (Congo). - Prout iu Seitz, l. Elfenbeinc., t. $5 \mathrm{k}$ (1933).

chaly beat a Swinh., Tr. Ent. Soc. Lond., 1904, p. 564 (1904). - Prout, Nov. Zool., 22 , p. 327 (1915).

fungifera Warr., Nov. Zool., 16, p. 113 (1909). - Kasai Prout in Seitz, 1. c., t. $5 \mathrm{k}$ (1933).

aplcipuncta Prout, Nov. Zool., 22, p. 327 (1915); Goldküste id. in Seitz, l. c., t. $5 \mathrm{k}$ (1933).

postlineata Warr., Nov. Zool., 6, p. 335 (1899) ? Indien (Leptomeris) ("India" [err. loc.?]). Hampson, Journ. Bomb. Nat. Hist. Soc., 14 : 4 p. 652 (1903) (Erythrolophus).

scdata Prout, Ann. Transv. Mus., 8:3, p. 155 Natal, (1922) (Natal); id. in Seitz, Macrolep., 16, Madagaskar p. 58 , t. 6 f (1933).

b a pta Warr. MIS. in Mus. Tring (Leptomeris) (Madagascar).

Iraus Prout, Nov. Zool., 23, p. 276 (1916); id. in UbangiSeitz, l. c., t. $5 \mathrm{k}$ (1933). Schari-

Tschad

lia Prout, Nov. Zool., 22, p. 327 (1915) (Comoro ComoroIs.); id. in Seitz, 1. c., t. $6 \mathrm{f}$ (1933). Inseln, Madagaskar

Ioscia Prout, Nov. Zool., $37: 2$, p. 238 (1932); id. Südin Seitz, l. c., t. $5 \mathrm{k}(1933)$. Rhodesia

centrofasciaria Leech, Ann. Mrag. Nat. Hist., (6) Zentral20, p. 100 (1897) (Acidalia). - Prout in China Seitz, Macrolep., 4, p. 45, t. 5 f (1913).

mendicaria Leoch, Ann. Mag. Nat. Hist., (6) 20, Zentral- u. p. 100 (1897) (Acidalia) (W. Ohina). - WestProut in Seitz, Macrolep., 4, p. 45, t. $5 f$ China (1913).

sublucens Warr., Nov. Zool., 14, p. 142 (1907) Holl.- u. (Organopoda) (Biagi) (huj. gen.?). guinea 
nucleata Warr., Nov. Zool., 12, p. 390 (1905) Sierra-Leone (Sierra Leone). - Prout, 'Tr. Ent. Soc. bis Lond., $1927: 1$, p. 189 (1927); id. in Seitz, Macrolep., 16, p. 58, t. $6 \mathrm{f}$ (1933).

Congo,

Sao-Thomé

indicataria Walk., List Lep. Ins., 23, p. 809 (1861) China, (Argyris) (N. China). - Butl., Ill. Het., Ost3, p. 43, t. 51, f. 8 (1879) (Argyris). - Sibirien, Graeser, Berl. Ent. Zeits., 32, p. 390 (1889) Korea, (Problepsis). - Leech, Tr. Ent. Soc. Lond., Japan 1889, p. 146 (1889) (Acidlatia); id., Ann. Mag. Nat. Hist.i, (6) 20, p. 110 (1897) (Dithatama). - Mey1., Tr. Ent. Soc. Lond., 1892 , p. 91 (1892) (Dithalama). - Stdgr.' Iris, 10, p. 21 (1897) (Acidalia); id., Cat. Iep. Pal., 1, p. 276 (1901) (Dithalama). - Püng., Iris, 10, p. 363 (1898) (Problepsis). - Swinh., Oat. Lep. Het. Oxf. Mus., 2, p. 375 (1900) (Dithalama). - Herz, Ann. Mus. St. Pét., 9, p. 338 (1905) (Dithalama). - Prout in Seitz, Macrolep., 4, p. 44, t. 5 a (1914); id., Nov. Zool., 35 : 4, p. 297 (1930).

vestalis Butl., Ann. Mag. Nat. Hist., (4) 16, p. 419 (1875) (Argyris) (Natal); id., Proc. Zool. Soc. Lond., 1894 , p. 593 (1894) (Problepsis). - Swinh., Tr. Ent. Soc. Lond., 1904, p. 564 (1904) (Problepsis). - Prout, Nov. Zool., 24, p. 430 (1917); id. in Seits, Macrolep., 16, p. 58, t. $6 \mathrm{~g}$ (1933).

virginalis Prout, 1. c. (1917) (Uganda); id. in Gambia, Seitz, 1. c., t. $6 \mathrm{~g}$ (1933).

? ves talis J. Joann. Bull. Soc. Ent. Ital., 44, p. 136 (1912) (Problepsis) (? err. det., nee Butl.).

Sierra-Leone.

Congo,

Ost-A frika,

Nyasa

mozambica Th.-Mieg, Le Nat., 27, p. 192 (1905) (Problepsis). - Prout in Seitz, 1. c. (1933).

eurymitra Turn., Tr. Roy. Soc. S. Austral., 50, p. Queensland 124 (1926).

omicraria F., Supp. Eut, p. 452 (1798) (India Coylon, orient.). - Ch. Auriv., Ent. Tidskr., 18, Indien, p. 165 (1897) (Problepsis). - Prout, Nov. Bali Zool., 22, p. 328 (1915 (- ata). -

q $e x t r u s u t a$ Walk., List Lep. Ins.b 22, p. 637 (1861) (Ephyra) (Ceylon). Swinh., Proc. Zool. Soc. Lond., 1885, p. 306 (1885), 1886, p. 441 (1856) (Argyris); id., Tr. Ent. Soc. Lond., 1894, p. 183 (1894) (Problepsis extrusaria); id., Cat. Lep. Het. Oxf. Mus., 2, p. 375 (1900) (Dithalama). Moore, Lep. Ceyl. 3, p. 539, t. 202, f. 7 (1887) (Problepsis). - Hampson, Il. Het. 8, p. 9 (1891); id., 1. c., 9, p. 39 (1893) (Problepsis); id., Faun. Ind., Moths 3, p. 463 (1895).

c a n a Hampson, Faun. Ind., Moths, 3, p. 463 (1895) (Punjab). 
Iriocellata Bastelb., Int. Ent. Zeits., 2, p. 33 (1908) Nord(Problepsis) (Cap-York) (pr. I.?).

Australien,

c a n a Turn., Proc. Linn. Soc. N. Sth. Wales,

? Sumba

$32: 4$, p. 675 (1908) (Problepsis). - Ch. Auriv., Ark. Zool., $13: 2$, p. 34 (1920)! (Problepsis) (nee Hampson?).

transveliens Prout, Nov. Zool., 25, p. 81 (1918); Hainan id., Bull. Hill Mus:, 4:1, p. 131 (1930).

pythiaria Guen., Spec. Gén. Tép., 10, p. 14, t. 20, Abyssinien f. 6 (1858) (Argyris). - Walk., List Lep. Ins., 23, p. 808 (1861) (Argyris). - Prout in Seitz, Iacrolep., 16, p. 5s, t. $5 \mathrm{k}$ (1933).

probleptica Prout, Nov. Zool., 24, p. 429 (1917); Nordid. in Seitz, 1. c., t. 5 i (1933).

figurata Warr., Nov. Zool.s, 4, p. 61 (1897) (Natal): ?Abyssinien, - Prout, Ḿ́m. Soc. Zool. Fr., 29:5, p. 395 (1932); id. in Seitz, t. c.v p. 59, t. $6 \mathrm{~g}$ (1933).

p y thiaria Wllgr., Ofv. K. Vet.-Akad. Förh., $32: 1$, p. 119 (1875) (Argyris) (vix Guen.).

ab. rufit a cta Warr., Nov. Zool., 12, p. 390 (1905) (Natal). - Prout in Seitz, 1. c. (1933).

var. candida Prout, Nov, Zool., $37: 2$, p. Uganda 238 (1932); id. in Seitz, l. c. (1933).

var. transfigurata Prout, Nov. Zool., 29, Madagaskar p. 336 (1922); id. in Seitz, l. c. (1933).

accraria Swinh., Tr. Ent. Soc. Lond., 1904, p. 565 Goldküste (1904) (pr. f.?). - Prout in Seitz, l. c. 42 (1933).

\section{Genus Problepsis Led.}

Ca lo pt er a Friv., K. Magyar Term. Tars. Evkon., 1, p. 185 (1846) (typus ocellata Friv.). - H.-Sch., Syst. Bearb. Schmett. Eur., 2, p. 97 (1846). - Berge, Schmetterlingsb. (ed. 2), p. 147 (1851) (nom. praeocc.).

Problepsis Ied., Verh. Zool.-Bot. Ver. Wien, 2, p. 74 (1852). - Stdgr., Cat. Lep. Eur., (ed. 2), 1, p. 152 (1871). - Moore, Lep. Ceylo; 3, p. 538 (1887). - Meyr., Tr. Ent. Soc. Lond., 1892 , p. 90 (1892). - Hampson, Faun. Ind., Moths, 3, p. 461 (1895). - Reb. in Berge, Schmetterlingsb., (ed. 9), p. 324 (1910). - Strand, Entom. Rundsch., 28, p. 122 (1911). - Prout in Seitz, Macrolep., 4, p. 49 (1913); 16, p. 59 (1933). - (nom. nor. pro Caloptera).

Argyris Guen., Spec. Gén. Lép., 10, p. 12 (1858). Walk., List. Lep. Ins., 23, p. 804 (1861). - Gmpbg., Nova Acta Acad. Leop. Nat., 49, p. 324, 342 (1887) (typus ommatophoraria Guen.).

Eue phyra Gmpbg., t. c., p. 328 (1887) (nec Pack.).

Problepsiodes Warr., Nov. Zool., 6, p. 336 (1899): (typus conjunctiva Warr.).

craspediata Warr., Nov. Zool., 4, p. 222 (1891); N.-O.10 , p. 370 (1903). 
var. langimacula Warr., Nov. Zool., Brit.-

10 , p. 370 (1903) (craspediata ab.). Neuguinea

var. rotifera Prout, Nov. Zool., 23, p. 17 Holl.-

(1916).

Neuguinea

ochripicta Warr., Nov. Zool., 8, p. 10 (1901) (S. Sao Thomé, Thomé). - Prout in Seitz, Macrolep., 16, Principe p. 59, t. 8 a (1933).

flavistigma Swinh., Tr. Fnt. Soc. Lond., 1904, p. Sierra Leome 561 (1904). - Prout in Seitz, 1. c. t. 8 a (1933).

ochripicta (part.) Warr., Nov. Zool., 8, p. 10 (1901) (nec typ.).

var. dilatistigma Prout, Nov. Zool., 24, Kenya p. 432 (1917); id. in Seitz, l. c., (1933).

rorida Plout, Nov. Zool., $37: 2$, p. 238 (1932); id. Nyasa in Seitz, l. c., t. 8 b (1933).

latonaria Guen., Spec. Gén. Iuép., 10, p. 14(1858) Caffraria (Argyris). - Walk., list Trep. Ins., 23, p. 807 (part.) (1861) (Argyris). Wligr., Ofv. Vet.-Al. Handl., $32: 1$, p. 119 (1875) (Argyris). - Ch. Oberth., Et. Iép. Comp., 20, p. 218, t. 550, f. 4651 (1923) (Arqyris). - Prout in Sieitz, 1. c., t. $8 \mathrm{~b}(1933)$.

milis J. Joann., Lép. Hét. Mascar. (Livre Cent. Mauritius Soc. Ent. Fr.), p. 438, t. 23, f. 10 (1932).

aegretta C. Feld., Reiss Novara, Lep. Het., t. 128, Natal, f. 14 (1875). - Prout, Nov. Zool., 24, p. Kapland 431 (1917); id. in Seitz, Macrolep., 16, p. p. 58, t. 8 a (1933). - Janso, Chook List S. Afr. Lep. Het, p. 95 (1917).

d igam mata Warr., Nov. Zool., 4, p. 59 (1S97) (nec W. F. Kirby, 1896).

var. insculpta Prout, l. c. (1917) (Ki- Uganda, kuyu Escurpment); id. in Seitz, 1. c. (1933). Kenya - aegretta (part.) Swinh., Tr. 1nt. Soc. Iond., 1904, p. 564 (1904). - egretta. Hale Carpt., Pioc. Ent. Soc. Lond., $7: 1$, p. 22 (1932).

B i o 1 o g i e: Hale Carpt., 1. c. (1932).

meroearia Saalm., Lep. Madag., p. 218, t. 5, f. 67 Madagaskar (1884). - Prout in Seitz, 1. c., t. 8 b (1933).

merocaria W. F. Kirby, Zool. Rec., 21; p. Ins. 216 (1885). - A. Pagenst. in Voeltzkow, Reise Ostafr., 2 : 2, p. 101 (1907).

var. (? syn.) mayottaria Ch. Oberth., 12t. Mayotte Lép. Comp., 20, p. 219, t. 550, f. 4652 (1923) (A.). - Prout in Seitz, l. c., t. 8 b (1933).

rigammata W. F. Kirby, Ann. Mag. Nat. Hists, Sierra Leone, (6) 18, p. 396 (1896) (Natal). - Karsch Uganda, in Werther, Mittl. Hochl. Deutsch-Ost-Afr., Konya bis p. 312 (1898). - Prout, Nov. Zool., 24, p. Griqua430 (1917); id., Mém. Soc. Zool. Fr., 29: land 
5, p. 395 (1932); id. in Seitz, l. c., t. 8 a (1933).

latonaria var. Walk., List Lep. Ins., 22, p. 807 (1861) (A.). - Butl., Ann. Mag. Nat. Hist., (4) 16, p. 419 (1875) (A.) (nec Guen.).

? pythiaria Wllgr., Ofv. Vet.-Ak. Förh., $32: 1$, p. 119 (1875) (A.) (exr. det., nec Guen.).

a e gretta (part.) Swinh., Tr. Ent. Soc.s Lond., 1904, p. 564 (1904) (Natal) (nec Feld.).

similinotala Prout, Nov. Zool., 24, p. 431 (1917) Fr.(Upper Congo); id. in Seitz, t. c., p. 60, Guinea, t. 8 a (1933).

Kamerun,

Congo,

? Nyasa

Neumanni Prout, Nov. Zool., $37: 2$, p. 238 (1932); Süd-Westid. in Seitz, l. c., t. 8 b. (1933).

Abyssinien

evanida Prout, (Treubia 7 , p. 425, 1929, nom. nud.) Buru Stylops 2, p. 26 (1933).

argentea Warr., Nov. Zool., 7, p. 107 (1900). Dammer-

Insel,

? Timor

clemens T. P. Luc., Proc. Linn. Sor. N. Sth. Wales, Queensland (2) 4, p. 1093 (1890). - Turn., Proc. Linn. Soc. N. Sth. Wales, $32: 4$, p. 674 (1908).

margaritata Warr., Nov. Zool., 3, p. 377 (1896).

plenorbis (Warr., MS.) Prout, Nov. Zool., 24, p. Sumatra 309 (1917); id., Bull. Hill Mus., $1: 2$, p. 261 (1922), $1: 3$, t. 25 , f. 13 (1924).

apollinaria Guen., Spec. Gén. Lép., 10, p. 555 Nord-Indien, (vice appollinaric Guen., t. c., p. 13) (1858) Birma, (A.) (Borneo). - Walk., List Lep. Ins., Sirm, 23, p. 809 (1861) (A.). - Meyr., Proc. Borneo, Linn. Soc. N. Sth. Wales, (2) 2, p. 838 Colobes, (1888). - Turn., Proc. Linn. Soc. N. Sth. Nouguinea, Wales, $32: 4$, p. 675 (1908). - Strand, Queensland, Iris, 1910 , p. 199. - C. Oberth., Et. Lép. BismarckComp., 20, p. 218, t. 549, f. 4648 (1923). Archipel a p pollinaria Swinh., Lep. Het. Oxf. Mus., (varr.?) 2, p. 375 (1900). - Prout, Nor. Zool, 24, p. 312 (1917). - Tams, Journ. Nat. Hist. Soc. Siam, $6: 3$, p. 274 (1924).

argentifila Warr., MS. (in Mus. Tring). var. he micyclata Warr., Nor. Zool., 4, Kei-Inseln, p. 59 (1897).

? Jobi

var. candidior Prout, l. c. (1917).

Süd-Indien

var. deparcata Prout, Nov. Zool., 32, p. 37 (1925).

St.-Matthias-

Insel

var. Wilemani West, Nov. Zool, $35: 2$, Luzon p. $255(1930)$. - ?deliaria G. Semp., Reisen Philipp., (2) $6: 6$ (1902) (noc Guen.). 
ocellala Friv., Magy. Temész. Tars. Evk., 1, p. Griechenland, 185 , t. 3, f. 1 (1845) (C:). - H.-Sch., Syst. Kreta,

Bearb. Schmett. Eur., 2, p. 97 (1846), t. Klein-Asien,

Bombyces, 23, f. 125, 126 (1848) (C.). - Syrien,

Led., Verh. Zool.-Bot. Ver. Wien, 2, p. 74 Cypern

(1852); id., Wien. Ent. Monats., 1, p. 99 (1857) (C.). - Walk., List Lep. Ins., 6, p. 1377 (1855) (C.); id., l. c., 23, p. 807 (1861) (A.). - Stdgr., Cat. Lep. Eur., p. 66 (1861); (od. 3) 1, p. 276 (1901); id., Hor. Soc. Ent. Ross., 7, p. 154 (1870). Snell., Tijdschr. Ent., 7, p. 198 (1864). Gmpbg., Nova Acta Acad. Leop. Nat., 49, p. $348(1887)$; ? 65 , t. 14, f. 1 (1896) (A.). - Meyr., Tr. Ent. Soc. Lond., 1892, p. 90 (1892). - Reb. in Spuler, Schmett. Eur., (ed. 3) 2, p. 27, t. 71 a, f. 22 (1903); id., Berge's Schmetterlingsb., (ed. 9), p. 324, t. 40, f. 5 (1910); id., Verh. Zool.-Bot. Ges. Wien, 65, p. (52) (1915); id., Ann. Hofmus. Wien, 30, p. 135, f. 'ّ (1916). Prout in Seitz, Macrolep., 4, p. 49, t. 5 a (1913). - Culot, Noct. \& Géom. Eur., 3, p. 86 , t. 13, f. 224 (1918).

o m matophor a ria Guen., Spee. Gén. Lép., 10, p. 13 (1858) (A.). - Millière, Ann. Soc. Linn. Lyon, 17, p. 51 (Icon. 2, p. 461), t. 98, f. 1 (1869) (A.).

var. (?) cinerea Butl.: Proe. Zool. Soc Nördl.

Lond., 1886, p. 387 , t. 35 , f. 8 (1886) Pandschab

(Argyria). - Warx., Proc. Zool. Soc. Lond., 1888, p. 298 (1888). - Hampson, Faun. Ind. Moths, 3, p. 462 (1895). - Prout, l. c. $(1913)$.

Morphologie: Led., 1. c. (1857).

metallopictala A. Pagenst., Jahrb. Nass. Ver. Nat., Amboina, 41, p. 174 (sep. p. 90) (1888) (A.) (Am- Ceram boina).

ve $n u s$ Th.-Mieg, Le Nat., 27, p. 192 (1905) (Amboina).

achlyobathra Prout, Bull. Hill Mus., $2: 1$, p. 47 Sumatra, (1928) (S. W. Sumatra). Perak

Iransposila Warr., Nov. Zool., 10, p. 370 (1903). Br.-

Neuguinea

vulgaris Butl., กll. Het, 7 , p. 43 , t. 125, f. 2 Indien, (1889) (Kangra). - Hampson, Ill. Het. Malay. 8, p. 9 (1891). - Prout in Sejtz, Macrolep., Halbinsel, 4, p. 50, t. 7 b (1913); id., Bull. Hill Mus. ? Tonkin, $4: 1$, p. 131 (1930).

de liaria Walk., List Lep. Ins., 23, p. 808 (1861) (A.). - ? Seitz, Macrolep 4,4 , t. 5 a Hainan, (1913) (non binom.) (err. det., nec Guen.). China

o cellata Moore, Proc. Zool. Soc. Lond., 1867 , p. 645 (1867); id., Journ. As. Soc. Bengal, 53 (2):3, p. 237 (1884). - Cotes 
\& Swinh., Cat. Moths Ind., 2, p. 187 (1887) (err. det., nec Friv.).

delphiaria ? Walk., List Lep. Ins., 23, p. 808 (1861) (A.). - Hampson, Faun. Ind., Motlis, 3, p. 462, f. 208 (1895). Leech, Ann. Mag. Nat. Hist., (6) 20, p. 109 (1897). - Swinh., Lep. Het. Oxf. Mus., 2, p. 375 (1900); id., Tr. Ent. Soc. Lond., 1902, p. 665 (1902). - ? Candèze, Encycl. Lép., $2: 3 / 4$, p. 119 (1927) (err. det., nec Guen.).

ab. a t te nu ata Warr., Nov. Zrool., 16, p. 126 (1909) (S. India).

albidior Warr., Nov. Zool:, 6, p. 33 (1899) (Kiulu). Nord-Indien, - Prout in Seitz, Macrolep., 4, p. 50 (1913) (deliaria f.); id., Ent. Mitt., 3, p. 240 (1914) (deliaria a.); id., Sar. Mus. Journ., $3: 2$, p. 177 (1926).

a pollinaria Butl., I1l. Het., 7, p. 7 (1889) (err. det.).

deliaria form. Swinh., ? Tr. Ent. Soc. Loud., 1890 , p. 191 (1890); id., 1. c., 1894, p. 183 (1894). - Haumpon, Fauu. Ind., Moths, 3, p. 462 (1895). - Leech, Ann: Mag. Nat. Histi, (6) 20, p. 110 (part.) (1897). - Matsumura, Ins. Mats., $4: 3$, p. 136 , t. 2, f. 20 (1930) (var.) (nec Guen.).

magna Warr., Nov. Zool., 13, p. 92 (1906) (Anga- Neuguinea bunga R.) (pr. f.?).

deliaria Guen., Spec. Gén. Lép., 10, p. 13 (1858) (A.) (Ceylon). - Walk., List Lep. Ins., 23 , p. 808 (part:) (1861) (Ceylon). Moore, Lep. Ceyl., 3, p. 539, t. 202, f. 6 (1887). - Hampson, Ill. Het.s, 8, p. 9 (1891); 9, p. 39 (1893); id, Faun. Ind., Moths, 3, p. 462 (part.) (1895). - Prout in Seitz, Hacrolep., 4, p. 50 (1913). - Ch. Oberth., Et. Ifép. Comp., p. 20, p. 218, t. 550 , f. 4650 (1923).

korinchiana Rothsch., Journ. F. M. S. Mus., $8: 3$, Westp. $139(1920)$.

Sumatra

cueireola Prout in Seitz, Macrolep., 4, p. 50, t. China $7 \mathrm{~b}$ (1913).

deliaria (part.) Ieech, Ann. Mag. Nat. Hist., (6) 20, p. 110 (1897) (nec Guen.).

paredra Prout, Nov. Zool., 24, p. 312 (1917). West-

China

maxima Th.-Mieg, Lo Nat., 27, p. 192 (1905) Japan (huj. gen.).

discophora Fixsen in Romff., Mém. Lép., 3, p. 348, China, t. 15, f. 4 (1887) (Korea). - Stdgr., Cat. Korea, Lep. Pal., 1, p. 276 (part.) (1901). - Japan Strand, Ent. Rundsch., 28, p. 122 (1911). Prout, Nov. Zool., 35:4, p. 298 (1930). - 
superans Leech, Ann. Mag. Nat. Hist., (6) 20 , p. 110 (part.) (1897). - ? Herz, Ann. Mus. St. Pét., 9, p. 339 (1905) (nec Butl.).

pho ebearia Matsumura, Thous. Ins. Jap., supp., 2, t. 27, f. 13 (1910) (err. det.).

superans Butl., Cist. Ent., 3, p. 122 (1885) (A.) Japan, (Japan). - Ieech, Ann. Mag. Nat. Hist., Ussuri, (6) 20 , p. 110 , (part.) (1897). - Strand, Korea, Ent. Rundsch., 28, p. 122 (1911). - Prout China in Seitz, Macrolep., 4, p. 50, t. 5 a (1913). - Matsumura, Ins. Mats., $4: 3$, p. 135, t. 2, f. $18(1930)$.

phoebearia Erschoff, Trukl. Soc. Ent. Ross., 4, p. 164, 197 (1870) (nom. nov.). - Gmpbg., Nova Acta Acad. Leop. Nat., $49: 4$, p. 349 (1887) (Euchyra). - Meyr., Tr. Ent. Soc. Lond., 1892, p. 90 (1892). - Stdgr., Iris, 10, p. 21 (1897); id., Cat. Lep. Pal., 1, p. 276 (1901). - Prout in Seitz, Macrolep., 4, p. 51, t. 5 a $(1913)$.

deliaria Brem., Lep. Ost.rSib., p. 79, t. 7 , f. 3 (1864) (A.) (nom. praeocc., nec Guen.) (Ussuri). - Strand, Ent. Rundsch., 28 , p. 122 (1911).

plagi a ta Butl., Tr. Ent. Soe. Lond., 1881, p. 22 (1881) (Japan).

longipannis Prout, Nov. Zool., 24, p. 310 (1917). Khasiberge

crassinolata Prout, l. c. (1917) (Khasis).

Sikkim, Assam, Tibet,

China

confuncliva Warr., Proc. Zool. Soc. Lond., 1893 p. 358 (1893) (Sikkim); id., Nov. Zool., 6, p. 336 (1899) (Problepsiodes). - Hampcon, Faun. Ind., Moths, 3, p. 463 (1895).

var. subjunctiva Prout, Nov. Zool., 24, Hainan

p. 309 (1917); id., Bull. Hill Mus., $4: 1$, p. 131 (1930).

delphiaria Guen., Spec. Gén. Lép., 10, p. 14 (1858) Birma bis (A.) ("Inde Centrale"). - Prout, Nov. Zool., Singapore, 24, p. 311 (1917). - Ch. Oberth., Et. Tonkin, Lép. Comp. 20, p. 218, t. 549, f. 4649 Sumatra, (1923). - J. Joann. Ann. Soc. Ent. Fr., Borneo, 98, p. 475 (1929). Palawan

argentisqu a ma Warr., Nov, Zool., 6, p. 337 (1899) (Problepsiodes) (Penang).

? de liaria Seitz, Macrolep., 4, t. 5 a (1913) (non binom.) (err. det., nec Guen.).

lucifimbria Warr., Nov. Zool, 9, p. 359 (1902). Celebes (Problepsiodes).

sancta Meyr., Proc, Linn. Soc. N. Sth. Wales, (2) 2, p. 839 (1888). - Turn 4 , Proc. Linn. Soc. N. Sth. Wales, $32: 4$, p. 675 (1908). Tillyard, Ins. Austral. \& N.Z., p. 452, t. 33, 37 f. 25 (1926). 


\section{Genus Lipomelia Warr.}

Warr., Proc. Zool. Soe. Lond., 1893, p. 359 (1. Aug. 1893). - Prout, Ann. Mag. Nat. Hist., (8) 6. p. 243 (1910).

De fo a Swinh., Ann. Mag. Nat. Hist., (6) 12, p. 151 (1. Aug. 1893).

Subusta Warr., Proc. Zool. Soc. Loud., 1893, p. Nord-Indien, 1893 , p. 360 , t. 32 , f. 24 (1893) (Sikkim). Birma, - Hampson, Faun. Ind., Moths, 3, p. 465 Formosa (1895) (Somatina). - Swinh., Lep. Het. Oxf. Mus., 2, p. 376 (1900) (Somatina). us ta ta Swinh., Ann. Mag. Nat. Hist., (6) 12, p. 151 (1893) (D.) (Khasia); id. Tr. 1

Ent. Soc. Lond., 1894, p. 182 (1894) (D.).

\section{Genus Nobilia Walk.}

Walk., List Lep. Ins., 24, p. 1098 (1862). - Swinh., Cat. Lep. Het. Oxf. Mus., 2, p. 377 (1900). - Prout, Nov. Zool., 22, p. 324 (1915); id., 1. c., 38:1, p. 1 (1932) (typus turbata Walk.).

Morphologie: Prout, 1. c. (1932).

obliterata Warr., Nov. Zool., 4, p. 220 (1897) (Bor- Borneo, neo). - Prout, Nov. Zool., 38:1, p. 2 Perak (1932).

cupreata A. Pagenst., Jahrb. Nass. Ver. Nat., 41, Amboina p 178 (1888) (Plutodes). - Prout, t. c., p. 3 (1932).

ne b u lo s a Warr., Nov. Zool., 4, p. 58 (1897). avellanea Prout, 1. c. (1932) ( $\mathrm{N}$. India). p. 650 (1867). - Candèze, Encycl. Lép., tu r b a ta Moore, Proc. Zool. Soc. Lond., 1867, 2:3/4, p. 118 (1927). - turbala (part.) Hampson, Faun. Ind. Moths, 3, p. 465 (1895) (Somatina). - Swinh., Lep. Het. Oxf. Mus., 2, p. 377 (1900). - Prout, Nov. Zool., 24, p. 307 (1917). - Rothsch., Proe. Ent. Soc. Lond., 1922, p. 132 (1923) (nec Walk.).

lurbala Walk., List Lep. Ins., 24, p. 1098 (1862) (Sarawak). - Hampson, l. c. (part.) (1895) (Somatina). - Strand, Iris, 1910, p. 199 (Somatina (Nobilia)). - Prout, Nov. Zool., 24 , p. 307 (part.) (1917); 38:1, p. 4 (1932); id., Sar. Mus. Journ., 3:2, p. 176 (1926); id., Journ. F. M. S. Mus., $17: 1$, p. 55 (1932). - Rothsch., l. c. (part.) (1923).

strigularia Snell. in Veth, Midden-Sumatra, 4:1 (2), p. 57 (1880) (Plutodes). - A. Pagenst., Jahrb. Nat. Ver. Nat., 41, p. 178 (1888) (Plutodes (Omiza)).

erotica Prout, Nov. Zool., $38: 1$, p. 4 (1932).

N.-Indien,

Birma, Malayische Halbinsel, Sumatra, Borneo, Formosa

Ostpegu, Teuasserim, Malayische Halbinsel, Sumatra, Java, Borneo, Mindanao, Mindoro

Celebes 
aphrodite Prout, t. c., p. 5 (1932) (Aroa River). Neuguinea, t ur b a ta (part.) Rothsch., 1 .c. (1923). D'Entrecasteaux, Ron, BismarekArchipel

strigata Warr., Nov. Zool., 3, p. 112 (1896) (Bor- Borneo, 7 neo). - Prout, l. c. (1932).

Malay.-

Halbinsel, Süd-Java

\section{Genus Ignobilia Prout}

Prout, Nov. Zool., $37: 2$, p. 238 (1932).

urnaria Guen., Spec. Gén. Lép., 9, p. 406 (1858) Malayische (Ephyra) (Borneo). - Oh. Oberth., Et. Lép. Halbinsel, Comp., 12, p. 150, t. 394, f. 3341 (1916), Sumatra, (Ephyra). - Prout, Nov. Zool., $37: 2$, p. Borneo, 1 239 (1932).

Palawan

\section{Genus Orthoserica Warr.}

Warr., Nov. Zool., 3, p. 113 (1896) (typus rufigrisea Warr.). Hampson, Journ. Bomb. Nat. Hist. Soc., 12 : 1, p. 87 (1898) (Somatina sect.'). - Prout in Seitz, Macrolep., 4, p. 415 (1915) (Somatini sect.).

rulfigrisea Warr., Nov. Zool., 3, p. 113 (1896) (Kha- Khasiberge, sia). - Hampson, Journ. Bomb. Nat. Hist. Malayische Soc., $12: 1$, p. 88 (1898) (S.). Halbinsel

var. mirandaria Leech, Ann. Mag. Nat, ZentralHist., (6) 19, p. 427 (1897) (Boarmia) Clina (Ichang). - Prout in Seitz, Macrolep., 4, 1 p. 415, t. 25 a (1915) (S.).

\section{Genus Craspediopsis Warr.}

Warr., Nov. Zool., 2, p. 93 (1895) (typus pallivittata Moore). Pront in Seit?, Macrolep., 4, p. 45 (1913).

Erythrolophus sect. 2B Hampson, Faun. Ind. Moths, 4, p. 563 (1896).

sinuosaria Leech, Ann. Mag. Nat. Hist., (6) 20, p. West108 , t. 8, f. 1 (1897) (Rhodostrophia). - China Prout in Seitz, 4, p. 45, t. 5 d (1913).

acutaria Leech, t. c., p. 91 (1897) (Acidalia). - Zentral- u. Prout, l. c., t. 5 e (1913).

China 
inaequata Warr., Nov. Zool., 3, p. 109 (1896): Nord-Indien (Khasia).

pallivittatus (part.) Hampson, Journ.

Bomb. Nat. Hist. Soc., $12: 1$, p. 87 (1898)

(E.) (err. det., nec pallivittata Warr.).

bimaculata Warr., Nov. Zool., 2, p. 94 (1895). - Khasiberge

Hampson, Faun. Ind., Moths, 4, p. 563 (1896) (E. pallivittatus nb.). - Swinh., Cat. Lep. Hot. Oxf. Mus., 2, p. 372 (1900).

b i punctata Warr., Nov. Zool., 3, p. 109 (1896) (en: transer.).

pallivittata Moore, Pros. Zool. Soc. Lond., 1867, Bengal, p. 641 (1867) (Anisodes) (Bengal). - Wa- Assam terh., Aid Ident. Ins, 1 , t. 151, f. 3 (1884) (Craspedia). - Swinh., 'Ir. Ent. Soc. Lond., 1894, p. 181 (1894) (Craspedia). - Swinh., Tr. Fint. Soe. Lond., 1894, p. 181 (1894) (Craspedia); Cat. Lep. Het. Oxf. Mus., 2, p. 371 (1900). - Warr., Nov. Zool., 2, p. 93 (1895).

pallivittatus Hampson, Faun. Ind., Moths, 3, p. 454 (1895); id., 1. c., 4, p. 563 (1896) (E.); id., Journ. Bomb. Nat. Hist. Soc., 12 : 1, p. 87 (1898) (E.).

rectata Walk., MS. (in coll. Druce) (Chorodna).

per'similis Moore, Lep. Coll. Atk., p. 254 (1888) Nond-West(Craspedia). - Warr., Proc. Zool. Soc. Lond., Indien 1888 , p. 322 (1888) (Craspedia); id., Nov. Zool., 3, p. 109 (1896). - Prout in Seitz, 6 Macrolep., 4, p. 45, t. 7 a (1913).

\section{Genus Discomiosis Prout}

Prout, Nov. Zool., 24, p. 324, 325 (1915) (typus anfractilinea Prout); id. in Seitz, Macrolep., 16, p. 60 (1933).

areioeentra Prout, Ann. Transv. Mus., $8: 3$, p. 155 Sǜ(1922) (Zululand); id. in Seitz, l. c., t. $6 \mathrm{~g}$ Rhodesia (1933).

crescentifera Prout, Nov. Zool., 22, p. 326 (1915). - Janse, Check List S. Afr. Lep. Het., p. 95 (1917) (err. det.).

crescentifera Warr., Nov. Zool., 9, p. 504 (1902) Ugogo, (Sterrha) (Barotse). - Prout, Ann. Transv. Barotse, Mus., 8:3, p. 156 (1922); id. in Seitz, Ma- Rhodesia crolep., 16, p. 60 , t. 51 (1933).

aniraetilinea Prout, Nov. Zool., 22, p. 325 (1915); Kenya irl., Móm. Soc. Zool. Fr., $29: 5$, p. 395 (1932); irl. in Seitz, l. c., t. $6 \mathrm{~g}$ (1933).

synnephes Prout, l. c. (1915); id. in Seitz, l. c., Kenya, 4 t. $6 \mathrm{~g}$ (1933). 


\section{Genus Tricentroscelis Prout}

Prout, Pros. Zool a Soc. Iond., 1916, p. 147 (1916); id., Mém. Soc. Zool. Fr., $29: 5$, p. 395 (1932); id. in Seitz, Macrolep., 16, p. 60 (1933).

protrusifrons Prout, Proc. Zool. Soc. Lond., 1916, Br.-

p. 147 (1916) (Brit. Somaliland); id., Mém. Somaliland, Soc. Zool. Fr., $29: 5$, p. 395 (1932); id. in Kenya 1

Seitz, l. c', t. $6 \mathrm{~g}$ (1933).

\section{9 bis. Genus Dualana Strand}

Strand, Archiv f. Naturg., 80 A, 1, p. 43 (1914) (typus veniliformis Strand); id., Entom. Nachrichtsbl., 1, p. 24 (1927).

veniliformis Strand, Archiv f. Naturg., $80 \mathrm{~A}, 1$, p. Kamerun 43 (1914) (Dualana) (Kamerun); id., Ent.

1 Nachrichtsbl., 1, p. 24 (1927).

\section{Genus Antitrygodes Warr.}

Warr., Nov. Zool., 2, p. 90 (1895) (typus divisaria Walk.). Swinh., Irep. Hret. Oxf. Mus., 2, p. 374 (1900). - Prout in Seitz, Macrolep., 16, p. 60 (1933).

Trygodes Hampson, Faun. Ind., Moths, 3, p. 460 (1895). - Turn., Proc. Linn. Soc. N. Sth. Wales, $32: 4$, p. 676 (1908) (ex err., nec Guen.).

cuneilinea Walk., List Lep. Ins., 26, p. $1752(1862)$ "India“, (Geometra). - Hampson, Faun. Ind. Moths, "Birma, 3, p. 461 (1895) (Trygodes). - Warr., Nov. Siam Zool., 3, p. 293 (1896). - Swinh., Lep. Het. Oxf. Mus., 2, p. 374 (1900).

dysmorpha Prout, Nov. Zool., 22, p. 329 (1915) Nigeria, (N. Nigeria); id. in Seitz, Macrolep., 16, Kamerun p. 60 , t. 51 (1933).

dentilinea Warr., Nov. Zool., 4, p. 47 (1897) (Sierra Sierra Leone Leone). - Hampson, Proc. Zool. Soc. Lond., bis 1910 , p. 477 (1910) (Trygodes). - Prout Gabun, in Seitz, 1. c., t. $6 \mathrm{~h} \mathrm{(1933).} \mathrm{Kenya,}$ Madagaskar

acinosa Prout, Nov. Zool., $37: 2$, p. 239 (1932); id. S. Thomé in Seitz, t. c., p. 61, t. 51 (1933).

dentilinea Prout, Tr. Ent. Soc. Lond, 1927 , p. 189 (1927) (err. det., nec Warr.). callibotrys Prout, Ann. Mag. Nat. Hist., (9) 1, Fr.p. 27 (1918) (Upper Kasai); id. in Seitz, Guinea, l. c., t. 51 (1933). Congo, Uganda, Madagaskar (ab. vel var.) 
vicina Th.-Mieg, Le Nat., 29, p. 271 (1907) (Try- Sikkim, godes) (Khasis) (sequ. var.?).

Assam,

? Südchina

agrata O. Felder, Reise Novara, Lep. Het., t. 128, Borneo, f. 19 (1875) (Trygodes) (,Molukken" Celebes, [? Celebes]).

restricta Warr., MS. (in Mus. Brit.) (Celebes).

?'divisaria G. Semp., Reisen Philipp., (2) $6: 6$, p. 634 (1902) (nec Walk.).

parvimacula Warr., Nov. Zool., 3, p. 293 (1896) Buru,

(Trobriand Is.). - Prout, Nov. Zool., 24, Obi, p. 308 (1917).

? agrata A. Pagenst., Jahrb. Nass. Ver. Nat., 41, p. 173 (1888) (Trygodes) (err. det., nec Feld.) (praec. var.?).

divisaria Turn., Proc. Linn. Soc. N. Sth. Wales, $32: 4$, p. 676 (1908) (Trygodes).

Amboina,

Key-Inseln,

Neuguinea,

D'Entre-

casteaux,

Trobriand-

Inseln,

Lusiaden,

Queensland,

? Neu-Meck-

lenburg

var. privativa Prout, Nov. Zool., 24, p. 307 Rook-Insel,

(1917) (Rook I.). - a g ra la A. Pagenst., Neu-

Zoologica, 12 (29), p. 149 (1900) (Trygodes) (err. det., nec Feld.).

pommern,

Admiralitäts-

Inseln

(var.?)

subaequalis Prout, Nov. Zool., 24, p. 308 (1917). Ysabel-

Insel

pirimacula Prout, Nov. Zool., 23, p. 16 (1916), Holl.- u. Brit (Dutch New Guinea). Neuguinea

divisaria Walk., List Lep. Ins., 23, p. 927 (1861) Indien, (Macaria) (Canara). - Moore, Proc. Zool. Malay.Soc. Lond., 1867, p. 642 (1867); id., l. ed, Haibinsel, 1877, p. 621 (1877) (Trygodes). - Butl., Sumatra, Proc. Zool. Soc. Lond., 1892, p. 132 (1892) Java, (? T.). - Hampson, Ill. Het., 9, p. 40 Borneo (1893) (Trig.); id., Faun. Ind., Moths, 3, p. 440,441 , f. 207 (1895) (T.). - Swinh., Tr. Ent. Soc Lond., 1894, p. 184 (1894) (T.);-id., Cat. Lep. Het. Oxt. Mus., 2, 374 (1900). - Prout, Ent. Mitt., 3, p. 3240 (1914); id., Journ. F. M. S. Mus., $17: 1$, p. 55 (1932). - Rothsch., Journ. F. M. S. Mus., $8: 3$, p. 139 (1920). - Candèze, Encycl. Ent. Lép., $2: 3 / 4$, p. 118 (1927) (Trig.). - J. Joann., Ann. So. Ent. Fr., 99, p. 475 (192)). var. perturba ta Piout, l. c. (1914).

Morphologie: Prout, t. c., p. 241 (1914). 


\section{Genus Paofa Hulst}

Hulst, Tr. Amer. Ent. Soc., 23, p. 295 (1896) (Typus fultaria Grote).

Iultaria Grote, Can. Ent., 14, p. 174 (1882) (Ly- Arizona thria). - Hulst, Tr. Amer. Ent. Soc., 23, p. 295 (1896). - Dyar, List N. Amer. Lep., p. 292 (1902). - Barmes \& Me Dunnough, Check List Lep. Bor. Amer., p. 103 (1917).

saruncaria Schaus, Tr. Amer. Ent. Soc., 27, p. 241 Mexiko

2 (1901) (Lythria?) (pr. var.?).

\section{Genus Stigma Alph.}

Alph., Hor. Soc. Ent. Ross., 17, p. 164 (1883). - Prout in Seitz, Macrolep., 4, p. 86 (1913).

Morphologie: Sterneck, Zeits. Osterr. Ent. Ver., 17, p. 70 (1932).

kuldschaensis Alph., 1. e., t. 8, f. 83, t. 9, f. 98 Kuldscha (1883); id. in Romff., Mém., 9, p. 50 (1897). - Meyr., Tr. Ent. Soc. Lond., 1892, p. 86 (1892) (Emmiltis). - Stdgr. \& Reb., Cat. Lep. Palaearct., 1, p. 278 (1901). - Prout in Seitz, Macrolep., 4, p. 87, t. 5, f. 9 (1913).

var. ne grita Th.-Mieg, Le Nat., 27, p. 103 Issyk-kul, (1905). - Prout, l. c. (1913). - atraria Alexander1 B.-Haas, Iris, 19, p. 137 (1906).

kette

\section{Genus Oar Prout}

Prout in Seitz, Macrolep., 4, p. 86 (1913).

Morphologie: Sterneck, Zeits. Osterr. Ent.-Ver., 17, p. 70 (1932).

pratana F., Ent. Syst., $3: 2$, p. 161 (1794), - Coque- Nordafrika, bert, IIl. Ie. Ins., 2 , p. 71 , t. 17, f. 6 a $-6 \mathrm{c}$ Südspanien, (1801) (Barbaria). - Weber, Nomencl. Ent., Südp. 132 (1795). - Rogenh., Verh. Zool.-Bot. frankreieh Ges. Wien, 25, p. 801 (1876) (Cleta (Acidalia)). - Bohatsch, Wien. Ent. Zeit., 4, p. 143 (1885) (Acidalia). - Stdgr. \& Reb., Oat. Lep. Pal., 1, p. 349 (1901) (F'idonia). Reb. in Spuler, Schmett. Eur. (E. Hofm. Großschmett., ed. 3) 2, p. 113 , t. 71 b, f. 15 (1905) (Fidonia). - Mab., Ann. Soc. Ent. Fr., 75, p. 34 (1906) (Fidonia). - Chrétien, Le Nat., 31, p. 54 (1909) (Fidonia). - Prout in Seitz, Macrolep., 4, p. 86, t. 7 e (1913). Culot, Noct. \& Géom. Eur., 4, p. 134, t. 64, f. $1274(1920)$ (Fid.). - Turati \& Zanon, 
$\Delta$ tti Soc. Ital. Sci. Nat., 61, p. 155 (1922). Turati, ibid., 63, p. 113 (1924). - Rothsch., Ann. Mag. Nat. Hist., (9) 16, p. 206 (1925). pratamaria Turt., Syst. Nat., 3, p. 246 (1802).

re au muraria Millière, Iconogr., 2, p. 2, t. 51, f. 1-3 (1864) (Cleta) (Montpellier)! (ab.?). - Stdgr., Cat. Lep. Eur., (ed. 2) 1, p. 145 (1871) (Acidalia). - Gmpbg:, Nova Acta Acad. Leop. Nat., 54, p. 540 (1890) (Cleta). - Meyr., Tr. Ent. Soc. Lond., 1892, p. 96 (1892) (Cleta).

megearia Ch. Oberth., Et. Ent., 6, p. 84, t. 3, f. 8 (1881) (Fidonia) (Oran). Meyr., T1. Ent. Soc. Lond., 1892, p. 86 (1892) (Emmiltis).

e ct y p a ta Mab., Bull. Soc. Ent. Fr., 1888, p. 58 (1888) (Oidaria) (Gabès, Tunisie).

var. oppressa Walk., Entom., 5, p. 154 Aegypten, (1870) (Ciduria) (Hor. Tamanib, Suakin). Sudan,

- obscuraria Beth.-Baker, Tr. Ent. ? Sinai, Soc. Lond., 1894, p. 45, t. 1, f. 12 (1894): Arabien (,Fid. megiaria var." (Alexandria). Stdgr. \& Reb., Cat. Lep. Pal., 1, p. 349 (1901). - Prout in Seitz, 1. c. (1913). nigrescens Hampson, Proc. Zool. Soc. Lond., 1896 , p. 267 , t. 10, f. 2 (1896) (Emmiltis) (Aden) (var. div.?). - ? prata n a Knencker, Verh. Naturw. Ver. Karlsruhe, 21, p. 138 (1909). - Reb. \& Zerny, Denks. Akad. Wiss. Wien, 93, p. 433 (1917).

- Andres \& Seitz, Senckenbergiana, 6, p. 73 (1924).

var. mortuaria Stdgr., Iris, $10: 2$, p. 314 Palästina (1898); id., Cat. Lep. Pal., (ed. 3) 1, p. 350 (1901). - Prout in Seitz, Macrolep., 4, p. 86 (1913). - Culot, l. c., f. 1275 (1920).

Biologie: Chrétien, l. c. (1909).

1

\section{Genus Dasybela Turn.}

Turn., Proc. Linn. Soc. N. Sth. Wales, $32: 4$, p. 667 (1908) (typus achroa Lower).

Dasybella Sharp, Zool. Rec., 45, p. 343 (1910).

achroa Lower, Tr. Roy. Soc. S. Austral., 26, p. 229 Tasmanien (1902) (Emmiltis). - Tum., Proc. Linn. Soc. N. Sth. Wales, $32: 4$, p. 667 (1908); id., Pap. \& Proc. Roy. Soc. Tas., 1925, p. 128 (1925).

argillina Lower, Tr. Roy. Soc. S. Austral., 39, p. Neu2 378 (1915) (Emmiltis ?) (huj. gen.?). Südwales 


\section{Genus Holarcfias Prout}

Prout in Seitz, Macrolep., 4, p. 85 (1913). - Barnes \& Mc Dunnough, Contrib. Lep. N. Amer., 3 : 3, p. 173 (1916).

Morphologie: Sterneck, Zeits. Osterr. Ent. Ver., 17, p. 70 (1932).

sentinaria Hb.-Gey., Zutr. Exot. Schmett., 5, p. Nördl. u. 9 , f. 823,824 (1837) (Haematopis) (Labra- westl. dor). - Guen., Spec. Gén. Lrép., 10, p. Canada 171 (1858) (Lythria?). - Walk., List Lep. Ins., 24 , p. 1056 (1862) (Lythria?). Stett. Ent. Zeit., 31, p. 368 (1870) (Acidalia). - Stdgr., Oat. Lep. Eur., (ed. 2), 1, p. 150 (1871), (ed. 3), 1, p. 272 (1901) (Acidalia). - Pack., Mon. Geom. U. S., p. 342 , t. 10, f. 61 (1876) (Acidulia). Gmpbg., Nova Acta Acad. Leop. Nat., 54 : 5, p. 542 (1890) (Cleta). - Meyr., Tr. Ent. Soc. Lond., 1892, p. 89 (1892) (Sterrha). - Hulst, Tr. Amer. Fint. Soc., 23, p. 303 (1896) (Leptomeris); id., Ent. News, 11, p. 529 (1900) (Emiltis). - A. Pagenst. in Roemer \& Schaudinin, Faun. Arct, $2: 2$, p. 305 (1902) (Acidalin). - Dyar, List N. Amer. Lep., p. 295 (1902) (Leptomeris)..Prout in Seitz, Macrolep., 4, p. 85 (1913). - Barnes \& Mo Dunnough, Oheck List Lep. Bor. Amer., p. 102 (1917).

s puriaria Christoph, Stett. Ent. Zeit., 19, p. 312 (1858) (Aspilates). - Möschl., Wien. Ent. Manats., 4, p. 372, t. 10 , f. $6,7(1860)$. (Acidalia). - Walk., List Lep. Ins., 26, p. 1593 (1862) (Acidalia) (Labrador).

gracilior Butl., Ann. Mag. Nat. Hist., (6) 12 , p. 16 (1893) (Thamnonoma) (W. (anada).

var. $r u f i n a r i a$ Stdgr., Cat. Lep. Eur., p. Sibirien 64 (1861) (Acidalia) (nom. nov.). - Gmpbg., Nova Acta Acad. Leop. Nat., $54: 5$, p. 530 (1890) (Cleta). - rufularia Eversm.; Bull. Mosc., $24: 1$ (2), p. 641 (1851) (Acid.) (nom. praeoce, nec H.-Sch., 1847). - Guen., Spec. Gén. Lép., 9, p. 450 (1858) (Acid.) (Sibérie or.). - Snell., Tijd, Ent., 6, p. 197 (1861) (Acid.). - Erschoff, Bull. Mosc., $43: 2(3 / 4)$ p. 315 (1871) (Acid.). $-r u$ fociliaria Brem., Mróm. Acad. St.-Pét., (7) 8 , p. 78, t. 7, f. 2 (1864) (Acidalia, (Ostsibirien). - Stdgr., Cat. Lep. Eur., (ed. 2) 1 , p. 146 (1871), (ed. 3) 1, 272 (1901) (A.); id., Iris, 10, p. 18 (1897) (A.). Hedem., Hor. Soc. Ent. Ross., 16, p. 243 (bis, recte 259] (1881) (A.). - Gmpbg., Nova Acta Acad. Leop. Nat., $54: 5$, p. 530 (1890) (Cleta). - Meyr., Tr. Ent. Soc. Lond., 1892, p. 89 (1892) (Sterrha). Herz, Iris, $11: 2$, p. 260 (1899) (A.); id., 
Ann. Mus. Zool. St.-Pét., 8, p. 84 (1903) (A.). - ruficiliaria Prout in Seitz, Macrolep., 4, p. 85 (1913).

var. rufinularia Stdgr., Cat. Lep. Pal., Sajan (ed. 3) 1, p. 272 (1901) (Sajan or. sum. (montic.) mont.). - Her\%, Ofv. Finska Vet.-Sac. Förh. $45: 15$, p. 13 (1902). - Prout in Seitz, 1. c. $(1913)$.

californiaria Pack., Proc. Bost. Soc. Nat. Hist., 13, Californien, p. 390 (1871) (Acidalia). - Barnes \& Mc ? Colorado Dunnough, Contrib. Lep. N. Amer., $3: 3$, p. 173 (1916); iid., Check List Lep. Bor. Amer., p. 102 (1917) (sequ. ab.?).

pacificaria Pack., Proc. Bost. Soc. Nat. Hist., 13, p. 391 (1891) (Acidalia). Gmpbg., Nova Acta Acaid. Leop. Nat., 58 : 4, p. 235 (1892) (Cnidia).

californiata Pack., Mon. Geom. U. S., p. 341 (1876) (Acidalia). - Butl., Parp., 1, p. 221 (1881) (Emmiltis). - Gmpbg., 1. c., (1892) (Cnidia).

magnetaria Guen., Spec. Gér. Lép., 9, p. 450 (1858) Westl.

(Acidalia) (Californie). - Hulst, Tr. Amer. Vereinigte Ent. Soc., 23, p. 303 (1896) (Leptomeris). Staaten - Dyar, List N. Amer. Lep., p. 295 (1902) (Leptomeris). - Barnes \& Mc Dunnough, Contrib. Lop. N. Amer., 3:3, p. 173 (1916); iid., Check List Lep. Bor. Amer., p. 102 (1917).

rubrolinearia Pack., Proc. Bost. Soc. Nat. Hist., 16, p. 28 (1874); id., Mon. Geom. U. S., p. 343 , t. 10 , f. 62 (1896) (Acidalia) (California).

rubrolineata Gmpbg., 1. c. (1892) /Cni3 dia).

\section{Genus Anfilycauges Prout}

Prout in Seitz, Macrolep., 4, p. 51 (1913).

pinguis Swinh., Tr. Ent. Soc. Lond,, 1902, p. 660 Tonkin, (1902) (Emmittis) (Formosa). - Prout in Hainan, Seitz, Macrolop., 4, p. 51, t. 5 b (1913); id., Süd-China, Ent. Mitt., 3, p. 241 (1914); id., Bull. Schanghai, 1 Hill Mus., $4: 1$, p. 131 (1930).

Chusan-

Inseln,

Formosa

\section{Genus Leucoxena Warr.}

Warr., Nov. Zool., 7, p. 94 (1900). - Prout in Seitz, Macrolep., 16; p. 61 (1933).

lactea Warr., 1. c. (1900). - Fawcett, Proc. Zool. Kenya

Soc. Lond., 1916, p. 731 (1916) (Induna).

- Prout, Mém. Soc. Zool. Fr., $29: 5$, p.

396 (1932); id. in Seitz, Macrolep., 16, p.

61 , t. $6 \mathrm{~g}$ (1933). 


\section{Genus Scopula Schrank}

Schrank, Fran. Boica, 2 : 2, p. 162 (1802). - Prout, Entom., 39, p. 266 (1906), 42, p. 4 (1909); id., Tr. City Lond. Ent. Soc.s 20, p. 25, 30 (1911); id., Nov. Zool., 23, p. 17 (1916); id.,' Ann. S. Afr. Mus., $17: 1$, p. 48 (1917); id., Ins. Samoa, 3:3, p. 128 (1928); id. in Seitz, Macrolep., 16, p. 61 (1933). - Janse, Check List S. Afr. Lep. Het., p. 95-97 (1917). - Derenne-Meyers, Faun. Belg., Géom., p. 11 (1929) (typus paludalis Schrank).

A o idalia Tr., Schmett. Eur., $5: 2$, p. 438 (1825), 6:2, p. 5 (1828). - Dup., Hist. Nat. Lép. Eur., $7: 2$, p. 108 (1829), 8:1, p. 70 (1830); id., Cat. Méth., p. 174 (part.) (1845). - Steph., I11. Haust., 3, p. 309 (1831). - Bsd., Gen. et Ind. Meth., p. 221 (part.) (1840). - Brd., Mém. Soc. Emul. Doubs., 2 : 2, p. 139 (sep. p. 121) (part.) (1846). - Heydenreich, Syst. Verz. Eur. Schmett., p. 15 (part.) (1856). - Staint., Man. Brit. Butt. \& Moths, p. 44 (part.) (1858). - Stdgr., Cat. Lep. Eur., p. 64-66 (part.) (1861); (ed. 2) 1, p. 150-152 (sect. B) (1871); (ed. 3) 1, p. 272276 (1901). - Walk., List Lep. Ins., 22, p. 680 seq. (part.) (1861). - Peyrhff., Cat. Lóp. Als., 1 (Bull. Soc. Colmar 2) p. 109-111 (part.) (1862); (ed. 2) 1, p. 112116 (part.) (1880). - Const., Cat. Lép. Saone-et-Loire. p. 200 (part.) (1866). - Rössler, Jahrb. Nass. Ver. Nat, $19 / 20$, p. 196 seq. (A. div. 2) (1866); 33/34, p. 168 (part.) (1881). - Snell., Vlind. Ned. Macrolep., p. 550 seq. (part.) (1867). - Berce, Faun. Ent. Franç., Lep., 5, p. 127 (part.) (1873). - Bang-Haas, Nat. Tidskr., (3) 9, p. 476-479 (part.) (1874). - Ramann, Sehmett. Deutschl., 4, p. 332 (part.) (1876). - Sp.-Schneid. in Siebke, Enum. Ins. Norveg., 3, p. 81, 82 (part.) (1876). - Gdl., Ent. Cubana, 1, p. 382 (1881) (Agidalia, err. typogr.). - Roüast, Ann. Soc. Linn. Lyon (n. s.) 29, p. 337, 338 (part.) (1883). - Bau, Haadb. f. Schmett.-Sammler, p. 232-235 (Acid. C) (1886). - Gmpbg., Nova Acta Acad. Leop. Nat., 49 : 4, p. 323-333 (part.) (1887); 54:5, p. 481-544 (part.) (1890); $58: 4$, p. 223-269 (part.) (1892). - Poppius, Aota Soc. F. F. Fenn., $3: 3$, p. 127-134 (purt.) (1887). - Meyr., Proc. Linn. Soc N. Sth. Wales, (2) 2, p. 536, 841 (part.) (1888). - V. Ström, Danm. Sommerf., p. 341 (part.) (1891). - Ch. Auriv., Nond. Fjär., p. 201, 205 (part.) (1891). Korb, Sclimett. Mittel-Eur., p. 170-175 (part.) (1894). - Kappel \& W. F. Kirb., Brit. \& Eur. Butt. u. Moths, p. 181-187 (part.) (1895). - Cholodkovsky, T. Hofm., Atlas Babochok Evr., p. 236, 240-243, t. 57, 58 (part.) (1897). Strand, Nyt Mag. Naturvid., 37, p. 53 (1899); id., 1. c., 39 , p. 30 (1901); id., 1. c., 40, p. 142,164 (1902). $\rightarrow$ Barr., Lep. Brit. Isl., 8, p. 1 (part.) (1901). - Rougemont, Cat. Lep. Jura (Bull. Soo. Neuchatel, 29 \& 31), p. 168-171 (part.) (1903). - A. Fuchs in Spuler, Schmett. Eur., (E. Hofm., GroBschmett., ed. 3) 2, p. 21-26 (part.) (1904). Strand, Nyt Mag. Naturvid., 42, p. 115, 138 (1904). - Prout, Entom., 42, p. 3 (1909); id. in Seitz, Macrolep., 4, p. 51 (1913). - Reb., Berge's Schmetterlingsb., (ed. 9), p. 319-324 (Acid. B) (1910). - Slastshersky, Hor. Soc. Ent. Ross., $40: 1$, p. 98-102 (part.) (1911). - Schawerda, Jahresb. Wien. Ent. Ver., 24, p. 140 (1914). - Blaschke, Raup. Eur., 
p. 4, 20, 25, 26, 85, 86, 131, 167 (1914). - Barnes \& Mc Dunnough, Oheck List Lep. Ber. Amer., p. 101 (1917). Hoffmann \& Klos, Mitt. Nat. Ver. Steier., 53, p. 56-78 (part.) (1917). - Strand, Archiv f. Naturg., 1919, A. 4, p. 5, 24. - Turn., Tr. Roy. Soa S. Austral., 46, p. 265 (1922). - Eckstein, Schmett. Deutschl., 4, p. 11-13 (part.), t. 49 , f. 4 (1923). - Chrétien, Amat. Pap., 4, p. 33, 49, 65, 81, 97 (1928). — Gaede, Tierwelt Deutschl., 14 (Schmett., 2 : Nacht.) p. 233-237, f. 144 (Acid, Gr. 2) (1929) (nom. praeocc., nec $\mathrm{Hb}, 1818$ ).

I d a e a (part.) Tr., Schmett. Eur., 5 :2, p. 446 (1825); 6 : 2, p. 258 (1828). - Children, Philos. Mag. (n. s.) 6, p. 464 (1829). - Ad. Spr., Isis, 1839, p. 110 (part.) (1839); 1843, p. 263, 264 (part.) (1843). - Heydenreich, Syst. Verz. Eur. Schmett., (ed. 2), p. 17 (1846), (ed. 3), p. 59 (1851) (part.). - Hocv., Handb. Dierk., (ed. 2) 1, p. 467 (1849). - Bertoloni, Nov. Comment. Bonon., 9, p. 171 (part.) (1849) (Idéa). - Butl., Proc. Zool. Lond., 1883, p. (1883). - Moore, Lop. Ceyl., 3, p. $452-455$ (part.) (1887). - Gmpbg., Nova Acta Acad. Leop. Nat., 54 : 5, p. 482, 485 (part.) (1890). - W. F. Kirby, Butt. \& Moths, Eur., p. 324 (part.) (1903) (typus floslactata Haw. $=$ remutata Sehiff., nec I.).

$L$ e $p$ t o meris Hb., Ver\%. Bek. Schnett., p. 310 (1826). Meyr., Tr. Ent. Soc. Lond., 1892, p. 89 (1892); id., Handb. Brit. Lep., p. 240 (1895); id., Tr. N. Z. Inst., 49, p. 264 (1917). - Hulst, Tr. Amer. Ent. Soc., 23, p. 303 (1896). Dyar, List N. Amer. Lep., p. 294, 295 (1902). - Prout, Entom., 38, p. 8 (1905). - Turn., Proc. Linn. Soc. N. Sth. Wales, $32: 4$, p. 655 (1908) (typus umbelaria Hb.).

Arrostia Hb., Verz. Bek. Schmett., p. 311 (1826). H.-Sch., Samml. AuBeneur. Sehmett., 1, p. 36 (1856). 'Heinem., Schmett. Dreutsclil., 1, p. 712 (1859). - Gartner Verh. Naturf. Ver, Brïnn, 4, p. 24 (1866) (Arrnostis). Fritsch, Denks. Akad. Wien, Math.-Nat. Cl., 41 :1, p. 94, 95 (1879). - Gmpbg., Nova Acta Acad. Leop. Nat., 49 : 5, p. 482, 494 (1890). - Prout, Entom., 38, p. 8 (1905); id., Tr. City Lond. Ent. Soc., 20, p. 26 (1911). - Linstow, Int. Ent. Zeits., 6, p. 42 (1912) (typus marginepunctata Göze = immutaria Hb.).

Craspedia Hb. (Zutr. Exot. Sohmett., 3, p. 33, 1825, indescr.) Verz. Bek. Schmett., p. 312 (1826). - Moore, Lep. Coyl., 3, p. 451 (1887). - Hampson, Faun. Ind. Moths, 3, p. 426 (1895). - Prout, Tr. City Lond. Ent. Soc., 20, p. 26 (1911) (typus ornate Scop.).

Dos ith e a Dup., Hist. Nat. Lép., $7: 2$, p. 208 (1829) ; 8 : 1, p. $43(1830)$; id., Diet. Univ. Hist. Nat., 5, p. $120(1844)$; id., Cat. Méth., p. 271 (18t5). - Brd., Mém. Soc. Emul. Doubs, 2 : 2, p. 139 (sep. p. 120) (1846). - Peyrhff., Bull. Soc. Colmar, 2, p. 108, 109 (1862) (typus ornata Scop.).

$C$ y $m$ a $t$ i $d$ a Sodoffsky, Bull. Mosc., $10: 6$, p. 91 (1837) (nom. nov., pro Acidalia).

Cy $m$ a to ide 8 Zell. in Agassiz, Nomencl. Zool., Index Univ., p. 112 (1846) (emend. pro Cymatida).

Pylarge H.-Sch., Samml. AuBereur. Schmett., 1, p. 36 (1856). - Heinem., Schmett. Deutschl., 1, p. 712 (1859). Fritsch, Denks. Akad. Wien, Math.-Nat. Ö., 41 :1, p. 94, 95 (1879) - Meyr., Handb. Brit. Lep., p. 244 (1895). Prout, Entom., 38, p. 8 (1905); id., Tr. City Lond. Ent. Soc., 
20, p. 26, 27, 32 (1911); id. in Seitz, Macrolep., 4, p. .52 (1913) (A., sect. P.); id., ed. german., p. 52 (A. soct. $P y$ larpe [err. typogr.]). - Turn., Proc. Linn. Soc. N. Sth. Wales, $32: 4$, p. 668 (1908). - Linstow, Int. Ent. Zeits., 6 , p. 42 (1912) (typus fumata Steph.).

Phyletis Guen., Spec. Gén. Lép., 10, p. 169 (1858). Warr., Nov. Zool., 4, p. 222 (1897). - Swinh., Tr. Ent. Soc. Lond., 1904, p. 560 (1904). L Prout, Mém. Soc. Zool. Fr., $29: 5$, p. 403 (1932) (typus silonaria Guen.).

Lycauges Butl., Ann. Mag. Nat. Hist., (5) 4, p. 373 (1879). - Moore, Lep. Ceyl., 3, p. 455 (1887). - Hampson, Faun. Ind. Moths, 3, p. 435 (1895) (Crasp., sect. II). Prout, Tr. City Lond. Ent. Soc., 20, p. 26, 31 (1911); id. in Seitz, Macrolep., 4, p. 53 (1913) (sub A. scct. Pylarge) (typus lactea Butl.).

Ca lothysanis Butl., Tr. Ent. Soc. Lond., 1881, p. 342 (1881) (err. det., neo $\mathrm{Hb}$. sect. typ.).

Trich o clada Meyr., Tr. Ent. Soc. Lond, 1886, p. 208 (1886); id., Proc. Linn. Soc. N. Sth. Wales, (2) 2, p. 838 (1888) (sub Problepsis). - Hampson, Faun. Ind., Moths 3, p. 426 (1895) (Crusp. syn.). - Prout, Ins. Samoa, $3: 3$, p. 128 (1928) (sub Scopula) (typus epigypsa Mryr.).

Run e c a Moore, Lep. Coll. Atk., p. 252 (1888) (typus ferrilineata Moore).

Cle ta (part.) Gmpbg., Nova Acta Acad. Leop. Nat., $54: 5$. p. 483,525 (1890) (nec Dup.).

Strania Gmpbg., 1. c., p. 483 (1890); $58: 4$, p. 268 (1892) (nec Dup.).

C nidia (part.) Grapbg., op. cit., 58:4, p. 223 (1892) (nec sect. typ.).

L ong u la Stdgr., Iris, 5, p. 157 (1892) (typus extraordinaria Stdgr.).

S yne lys Hulst, Tr. Amer. Ent. Soc., 23, p. 300 (1896). Dyar, List. N. Amer. Lep., 292 (1902) (typus enucleata Guen.).

A cidalina Stdgr., Iris, $10: 2$, p. 269 (1898)i (typus decolor Stdgr.).

In $d$ u n a Warr., Nov. Zool., 4, p. 55 (1897) (typus rufisalsa Warr.). - Janse, Check List S. Afr. Lop. Het., p. 07 (1917).

Pleionocentra Warr., Nov. Zool., 5, p. 242 (1898) (typus plionocentra Prout = minorata Warr., err. det.).

Chlorocraspedia Warr., Nov. Zool., 6, p. 292 (1899) (typus ansorgei Warr.).

T $i$ ma nd ra Barr., Lep. Brit. Isl., 8, p. 72 (1901) (err. det., nec Dup.).

E mm ilt is Warr., Nov. Zool., 8, p. 23 (1901). - Swinh., Tr. Ent. Soc. Lond,, 1904, p. 554 (1904) (nec Hb., H.-Sch! restr.).

Lipocentris Warr., Nov. Zool., 12, p. 389 (1905) (typus rubriceps Warr.).

Psile phyra Bastolb., Int. Ent. Zeits., 3, p. 101 (1909) (typus bilineala Bastelb.).

E o is Mosher, Bull. Ill. State Lab. Nat. Hist., $12: 2$, p. 128, 131 (1916) (nec Hb.).

U s to c idalia (part.) Sterneck, Zeits. Öst. Ent. Ver.; 17 : $9 / 10$, p. 70 (1932). 
Morphologie: Swinton, Soc. Fint., 23, p. 148 (1908). Linstow, Int. Ent. Zeits., 6, p. 42 (1912). - Pierce, Genit. Geom. Brit., p. $32-35$, t. 21, 22 (1914). - Mosher, 1. c.: (1916) (Eois). - Sterneck, Zeits. Ost. Ent. Ver., $17: 9-12$, p. $70,77-84$, t. 7 (1932); $18: 1$, p. $1-6,18: 2$, p. $9-14$ (1933).

B i o lo g i e: Fenn \& Buckell, Ent. Rec., 3, p. 175, 255 (1892). - Blaschke, 1. c. '(1914) (Acidalia). - Chrétien, 1. c. (1928) (Acidalia). - Boldt, Int. Ent. Zeits., 23:24, p. 286, 287 (1929).

Phänologie: Fritsch, 1. c. (1879) (Arrhostia \& Pylarge).

rufisalsa Warr., Nov. Zool., 4, p. 55 (1897) (In.) Rhodesia (Natal). - Hampson, Proc. Zool. Soc. Lond., bis 1910, p. 477 (1910) (In.). - Pront, Mém. Griqualand Soc. Zool. Fr., $29: 5$, p. 397 (1932); id. in Seitz, Macrolep., 16, p. 61, t. 6 h (1933).

exiguaria (part.) Swinh., Tr. Ent. Soc. Lond., 1904, p. 560 (1904) (In.) (err. det., nec: Walk.).

var. pallidisals a Prout, I. c. (1932); id. in Kenya, Seitz, 1. c. (1933). - exiguaria (part.) Tanganjika Swinh., I. c. (1904) (err. det., nec Walk.).

palpilera Prout, Ann. S. Afr. Mus., 19, p. 583, t. 17, Süd-Westf. 26 (1925); id. in Seitz, 1. c., t. $6 \mathrm{~h}$ (1933). Afrika

pliyletis Prout, Ann. 'Transv. Mus., $3: 4$, p. 197, t. Transvaal 12, f. 34 (1913) (Lyc.); id. in Seitz, t. c., p. 62, t. 6 h (1933). - Janse, Check List S. Afr. Lep. Het., p. 97 (1917).

molaris Prout, Ann. Transv. Mus., 8:3, p. 156 Süd(1922); id. in Seitz, l. c. (1933). Rhodesia

monotropa Prout, Ann. S. Afr. Mus., 19, p. 581, t. Süd-West17 , f. $24(1925)$; id. in Seitz, 1. c., t. $6 \mathrm{~h}$ Afrika (1933).

palleuca Prout, t. c., p. 582, t. 17, f. 19 (1925) (S. Süd-WestW. Africa); id. in Seitz, t. c., p. 61, f. 6 h Afrika, (1933).

N'Gami

curvimargo Warr., Nov. Zool., 7, p. 92 (1900) (In.) Rhodesia (N. Rhodesia). - Mampson, Proc. Zool. Soc. bis Lond., 1910, p. 477 (1910) (I.). - Lo Cerf Kenya, in Voy. M. Rothsch., Lep. Het., p. 443 (1922) ? Transvaal (I.). - Prout, Mém. Soc. Zool. Fr., $29: 5$, p. 397 (1932); id. in Seitz, t. c., p. 62, t. 6 h (1933).

bilineata Bastelb., Int. Ent. Zeits., 3, p. 101 (1909) Nyasa, (Psil.) (Nyasa). - Prout in Seitz, 1. c. Tanganjika, (1933).

Rhodesia

ab. $n$ u bicincta Hampson, Proc. Zool. Soc. Lond., 1910, p. 477 (1910) (In.) (N. E. Rhodesia).

pelloniodes Prout, Ann. Trans. Mus., 8:3, p. 156 Oranje(1922); id. in Seitz, 1. c. (1933):. 
albida Warr., Nov. Zrool., 6, p. 294 (1899) (In.) Sierra-Ieone (Usoga). - Prout in Seitz, 1. c., t. $6 i$ bis (1933). p u ra Swinh., Ann. Mag. Nat. Hist., (8) Uganda 3 , p. 94 (1909) (In.) (Uganda). - candida Warr. MS. in Mus. Tring.

cineraria Leech, Ann. Mar. Nat. Hist., (6) 20, p. Korea, 102 (1897) (Ac.) (Japan). - Prout in Seitz. Japan, Macrolep., 4, p. 52, t. 3k (1913) (Ac.); id.; RiukiuNov. Zool., 35:4, p. 298 (1930). - Ster- Inseln neck, Iris, 42, p. 137 (1928) (Ac.).

impersonata Walk., List Lep. Ins., 23, p. 758 (1861) China, (Ac.) (Foochow). - Leech, Ann. Mag. Nat. Süd-Japan Hist., (6) 20, p. 101 (1897) (Ac.). Swinh., Cat. Iep. Het. Oxf. Mus., 2, p. 362 (1900) (Cr.). - Prout, Tr. City Lond. Ent. Soc., 20, p. 31 (Pyl.); id. in Seitz, Macrolep., 4, p. 52, 53, t. $3 \mathrm{k}$ (1913) (Ac.). - Sterneck, Iris, 41:2, p. 155 (1927) (Ac.). var. macescen 8 Butl., Ann. Mag. Nat. Hist., (5) 4, p. 439 (1879) (Ac.). (Japan). Leech, t. c., p. 103 (1897) (Ac.). - Prout in Seitz, Macrolep., 4, p. 53, t. $3 \mathrm{k}$ (1913); id., Nor. Zool., 35:4, p. 298 (1930).

var. a ceurataria Christoph, Bull. Mose., Amur $55: 2$, p. 47 (1881) (Ac.). - Graeser, Berl. Ent. Zeits, 32:2, p. 384 (1889) (Ac.). Gmpbg., Nova Acta Acad. Leop. Nat., 54 : 5 , p. 529 (1890) (Cleta). - Stdgr., Iris, 10, p. 18, t. 1, f. 12 (1897) (Ac.?). - Prout in Seitz, Nacrolep., 4, p. 53, t. $3 \mathrm{k}$ (1913). Sterneck, Zeits. Osterr. Ent.-Ver., 17, p. 78 (1932) (Ac.).

ab. (?) m и scularia Stdgr., l. c., f. 13

(1897) (Ac.?) (Amur). - Prout, Tr. City Iond. Ent. Soc., 20, p. 32 (1911) (Pyl.); id. in Seitz, Macrolep., 4, p. 53 (1913).

gastonaria Ch. Oberth., Et. Ent., 1, p. 60, t. 3, f. Algerien 7 (1876) (Eubotia) (Oran). - Stdgr. \& Reb., Cat. Lep. Pal., 1, p. 355 (1901) (Eubolia). - Prout in Seitz, Macrolep., 4, p. 53 (1913), p. 416 (1915) (Ac.). - Culot, Noet. \& Geom. Eur., 4, p. 149, t. 68, f. 1355 (1920) (Eubotia).

ga sto naria var. ob s curaria Bang-Haas, Iris, 24, p. 49 (1911) (Eubolia).

ab. candicans Prout in Seitz, l. c., t. $5 \mathrm{~b}$ (1913); p. 417 (1915). - gastonaria B.Haas, 1. c. (1911). - Culot, t. c., t. 67, if. 1340 (1920). - hollaria (Ch. Oberth., M.S.) Culot, t. c., p. 149 (1920).

var. I u te of a sciata Rothsch., Nov. Zool., Süd20, p. 132 (1913) (Ac.). - Prout in Seitr, Algerien Macrolep., 4, p. 417 (1915) (Cleta?).

picta Warr., Nov. Zool., 4, p. 62 (1897) (Sterrha) Transvaal (Weenen), - Janse, Check List S. Afr. bis Lep. Het., p. 97 (1917). - Prout in Seitz, Kalahari, Macrolep., 16, p. 62 (1933). 
ab. fulvilinea Warr., Ann. S. Afr. Mus., $10: 12$, p. 483 (1914) (Sterrha) (Zululand). - Prout, 1. c., f. 6 i (1933).

impieta (Warr. M.S.) (Janse, Chock List S. Afr. Transvaal, Lep. Het., p. 96, 1917, nom. nud.) Prout, Basutoland, Ann. Transv. Mus., $8: 3$, p. 157 , t. 1, f. 9 Kapland (1922) (Transvaal); id. in Seitz, l. c., t. 6 i (1933).

flexio Prout, Ann. S. Afr. Mfus., $17: 1$, p. 48 Kapland (1917); $19: 4$, t. 16 , f. 2 (1925); id. in Seitz, l. c., t. 6 i (1933).

obliquiseripta Warr., Nov. Zool., 4, p. 62 (1897) Transvaal, (Sterrha) (Natal). - Prout, Ann. Transv. Natal Mus., $3: 4$, p. 126 (1913) (Pyl.).

gazellaria Janse, Check List S. Afr. Lep. Het., p. 96 (1917). - Prout, Bull. Soc. Lép. Genève, $6: 1$, p. 22 (1928); id. in Seitz, t. c., p. 63, t. 6 i (1933) (err. det., nec Wilgr.).

promethes Prout, Bull. Soc. Lép. Genève, 6:1, p. Transvaal, 22 (1928) (Mitt. Schweiz. Ent. Ges., 14:3, Kapland Beilage, p. 22, 1929) (S. (Pyl.)) (Basutoland); id. in Seitz, Macrolep., 16, p. 63, t. 6 i (1933).

sevandaria Swinh., Tr. Ent. Soc. Lond., 1904, p. Uganda, 553 (1904) $O$ (Lycauges) (Br. E. Africa). Kenya - Prout, Mém. Soc. Zool. Frr., 29 : 5, p. 399 (1932); id. in Seitz, l. c., (1933).

a peararia Swinh., t. c., p. 556 (1904) (Emm.) (Br. E. Africa). - Prout in Seitz, l. c., t. ol (1933).

acyma Prout, Mém. Soc. Zool. Fr., $29: 5$, p. 399 Kenya (1932); id. in Seitz, 1. c., t. 81 (1933).

teclnessa Prout, t. c., p. 398 (1932); id. in Seitz, Kenya 1. c. (1933).

subobliquata Prout, $\Lambda$ nn. Transv. Mus., $3: 4$, p. Transvaal 198 , t. 12 , t. 5 (1913) (Pyl.); id., Bull. Soc. Lép. Genève, $6: 1$, p. 22 (1928); l. c., t. 81 (1933).

gazellaria Wilgr., Wien. Ent. Monats., 7, p. 151 Transvaal (1863) (Ac.) (Caffraria or.); id., Ofv. Vet. bis Ak. Förh., $29: 1$, p. 54 (1872) (Ac.).

gazella Dist., Nat. in Tranv., p. 240 (1892) (Ac.) (err. transcr.).

punctilineata Warr., Nov. Zool., 4, p. 63 (1897) (Sterrha) (Natal). - Janse, Check List S. Afr. Lep. Hot., p. 97 (1917). Prout in Seitz, 1. c., t. $6 \mathrm{i}$ (1933).

ab. griseolineata Warr., Nov. Zool., 7, p. 94 (1900) (Sterrha) (Natal). - Prout, l. c. (1933).

ab. fus cut a Prout, 1. o. (1933).

concurrens Warr., Nov. Zool., 4, p. 57 (1897) (Ly- Nord-Ostcauges). - Prout in Seitz, l. c., t. $6 \mathrm{i}$ (1933). Rhodesia,

'Kenya

anaitisaria Walk., List Lep. Ins., 23, p. 795 (1861) Sürl-Indien (Ac.?). - Hampson, Ill. Het., 8, p. 30 (1891) (Lyc.); id., Faun. Ind., Moths, 3, 
p. 435 (1895) (Cr.). - Swinh., Cat. Lep. Het. Oxf. Mus., 2, p. 364 (1900) (Lyc.).

anaitaria Prout, Nov. Zool., 29, p. 337 (1922) (err. transcr.).

dimoera Prout, l. c. (1922).

de fa mataria Hampson, Ill. Het., 8, p. 30 (1891) (Lyc.); id., Faun. Ind., Moths, 3, p. 435 (1895) (Cr.) (err. det.).

emissaria Walk., List Jep. Ins., 22, p. 751 (1861) Ceylon, (Ac.) [Moulmein]. - Swinh., Tr. Ent. Soc. Indien Lond., 1890, p. 213 (1890) (Id.). - Hamp- Formosa, son, Faun. Ind., Moths, 3, p. 435 (1895) $(C r$.$) ; id., Journ. Bomb. N. H. Soc., 12$ : 1, p. 83 (1898) (Cr.). - Prout, Nov. Zool., 29, p. 337 (1922); id., Bull. Hill Mus., 4:1, p. 131 (1930) - Tums, Journ. N. H. Soc. Siam, 6, p. 274 (1924) (Lyc.). - Candèze, Encycl. Lép., $2: 3 / 4$, p. 118 (1927) (Lyc.). - J. Joann., Ann. Soe. Ent. Fr., 98, p. 478 (1929).

defamataria Walk., List Lep. Ins., 22, p. 752 (1861) (Ac.) (Ceylon). - Swinh., Proc. Zool. Soc. Lond., 1885 : 4, p. 857 (1886) (Ac.); id., Cat. Lep. Het. Oxf. Mus., 2, p. 364 (1900); id., Tr. Ent. Soc. Lond., 1902, p. 631 (1902) (Lyc.). - Moore, Lep. Ceyl., 3 , p. 456, t. 102, f. 2 (1887) (Lyc.). Hampson, Ill. Het., 9, p. 40 (1893) (Lyc.). - G. Semp., Reisen Philipp., (2) 6:6, p. 631 (1902) (Lyc.).

ab. mollis Warr., Nov. Zool., 3, p. 373 (1896) (Lyc.) (Khasia).

var. l a c te a Butl., Ann. Mag. Nat. Hist., (5) Japan, 4, p. 373 (1879) (Lyc.) (Japan). - Pryer, China

Tr. As. Soc. Jap. $12: 2$, p. 67 (1884) (Lyc.). (pr. p.) - Prout, Tr. City Lond. Ent. Soc., 20, p. 31 (1911) (Lyc.); id. in Seitz, Macrolep., 4, p. 54, t. $3 \mathrm{~g}$ (1913) (Ac.). - Hori, Dobuts. Zasshi Tokyo, 38, p. 32 (1926) (Ac.). Marumo, Joum. Coll. Agric. Tokyo, $8: 2$, p. 157 (1927). - ? Sterneck, Iris, 41, p. 156 (1927) (Ac.). - e miss a ria Leech, Ann. Mag. Nat. Hist., (6) 20, p. 103 (1897) (Ac.). var. proxima Butl., Tr. Ent. Soc. Iond., Queensland, 1886, p. 435 (1886) (Lyc.) (Queensland). - NeuTurn., Proc. Linn. Soc. N. Stl. Wales, $32: 4$, Südwales p. 669 (1908) $\left(P_{y} l.\right)$.

Philippinen,

Celebes,

Sumatra

bis

Sumbawa

Biologie: Prout, 1. c. (1911).

Donovani Dist., Nat. in Transv, p. 240, t. 2, f. 4 Marokko, (1892 Apr.) (Lyc.) (Transvaal). - Prout in Aegypten, Seitz, Macrolep., 4, p. 54 (1913), 16, p. 63, Syrien, t. 6 i (1933). - Janse, Check List S. Afr. Nigerien, Lep. Het., p. 96 (1917) (denovani, err. Uganda, typogr.).

Barotje,

e $x$ tra o rdina ria Stdgr., Iris, 5, p. 157, t. Transvaal, 2, f. 7 (1892 Jun.) (Long.) (Syrien). - Natal, Andres, Bull. Soc. Ent. Egypt., $1912: 3$, p. Madagaskar 
97 (1913) (Long.). - Prout in Seitz, Macrolep., 4, t. $3 \mathrm{~g}$ (1913) (Ac.).

e $x$ tr e m a t a Warr., Nov. Zool,, 4, p. 57 (1897) (Lyc.) (P. Niger).

orthoscia Meyr., Proc. Linn. Soc. N. Sth. Wales, West-

(2) 2, p. 861 (1888) (Ac.). - Turn., ibid.; Australien $32: 4$, p. 670 (1908) (Pyl.).

loxosema 'I'urn., t. c., p. 669 (1908) (Pyl.). Victoria

Iersicallis Prout, Tr. Ent. Soc. Lond,, $77: 2$, p. Marquesas267 (1929).

oxystoma Prout, t. c., p. 266 (1929).

Marquesas-

Inseln

crebospila Lower, Tr. lloy. Soc. S. Austral., 26, p. Queensland 250 (1902) (Pyl.). - Turn., Proc. Linn. Soc.

N. Sth. Wales, $32: 4$, p. 668 (1908) (Pyl.).

episcia Meyr., Proc. Linn. Soc. N. Sth. Wales, (2) West-

2, p. 863 (1888) (Ac.) (W. Australia). - Australien,

Turn., ibid., $32: 4$, p. 668 (1908) (Pyl.). Neu-

Südwales

megalocentra Meyr., t. c., p. 862 (1888) (Ac.). - Süd-

Turn., 1. c., p. 670 (1908) (Pyl.) (huj. Australien gen.?).

Andresi Draudt, Int. Ent. Zeits., 5, p. 374 (1912) Aegypten

(Ac.). - Prout in Seitz, Macrolep, 4, p. 54 (1913) (Ac.).

dapharia Swinh., Tr. Fnt. Soc. Lond., 1904, p. 552 Kenya, (1904) (Lyc.). - Le Corf in Voy. M. VictoriaRothsch., Lép. Hét., p. 447 (1922) (Lyc.). See

- Prout, Mém. Soc. Zool. Fr., 29 :5, p. 400

(1932); id. in Seitz, Macrolep., 16, p. 63,

t. $6 \mathrm{k}(1933)$.

neophyta Prout, Nov. Zool., 29, p. 337 (1922) (S. Columbien (Pyl.)).

aibidulata Warr., Nov. Zool., 4, p. 432 (1897) (Cr.) Süd-Ost-

biancaria Schaus, MS. in coll. E. D. Jones Brasilien (1d.).

anccllata Hulst, Ent. Amer., 2, p. 186 (1887) (Ac.) Felsen(California); id., Tr. Amer. Fnt. Soc., 23, Gebirge p. 301 (1896) (Cinglis). - Dyar, List N. Amer. Lep., p. 294 (Cinglis). - Dod, Can. Ent., 38, p. 256 (1906) (Cinglis). - Taylor, ibid., 40, p. 85 (1908) (Cinglis). - Barnes \& Mc Dunnough, Check List Lep. Bor. Amer., p. 102 (1917) (Ac.). - Bluckmore, Check List Macrolep. Brit. Col., p. 34 (1927). var. (?syn.) catenes Druce, Biol. Centr.- Mexiko Amer., Lep. Het., 2, p. 120 , t. 52 , f. 22 (1892) (Ac.).

fuscata Hulst, Ent. Amer., 2, p. 187 (1887) (Ac. Felsenquinquelineata var.); id., Tr. Amer. Ent. Gebirge Soc., 23, p. 301 (1896) (Cinglis) (Arizona). - Dyar, List N. Amer. Lep., p. 294 (1902) (Cinglis). - Barnes \& M[o Dunnough, Check List Lep. Bor. Amer., p. 102 (1917) (Ac.). 
- Blackmore, Check List Macrolep. Br. Col., p. 34 (1927).

Iutcolata Hulst, Bull. Brookl. Ent. Soc., 3, p. 42 Colorado (1880) (Cidaria); id., Ent. Amer., 2, p. 222 (1887) (Ac.); id., Tr. Amer. Ent. Soc., 23, p. 301 (1896) (Cinglis). - Barnes \& Mc Dunnough, Check List Lep. Bor. Amer., p. 102 (1917) (Ac.).

Elwesi Prout, Bull. Hill Mus., $1: 2$, p. 261 (1922); Altai $1: 3$, p. 624, t. 25, f. 10 (1924).

ansulata Led., Hor. Soc. Ent. Ross., 8, p. 19, t. 2, Nord-Persien f. 6 (1871) (Ac.). - Gmpbg., Nova Acta Acad. Leop. Nat., $54: 5$, p. $497^{\prime}(1890)$ (Ar.). - Meyr., Tr. Ent. Soc. Lond., 1892, p. 89 (1892) (Lept.). - Stdgr. \& Reb., Cat. Lep., Pal., 1, p. 275 (1901) (Ac.). - Prout in Seitz, Macrolep., 4, p. 55 (1913) (Ac.).

var. a du l teraria Ersch., Lep. Fedchenko's Caspi-See Roise, p. 60, t. 4, f. 61 (1874) (Ac.) (Tur- bis Ili kestan). - Christoph in Romft., Mém. Iép., 5, p. 48 (1889) (Ac.). - Stdgr., Iris, N, p. 157 (1892) (Ac.); id., Cat. Lep. Pal, 1, p. 275 (1901) (Ac.). - Meyr., Tr. Ent. Soc. Lond., 1892, p. 69 (1892) (Lept.). - Prout in Seitz, Macrolep., 4, p. 55, t. 41 (1933).

ab. $c h a r a c t e r i s t i c a$ Alph., Hor. Soc. Ent. Ross., 17, p. 162, t. 9 , f. 87 (1882) (Ac.) (Kuldscha). - Stdgr., Iris, 5, p. 157 (1892) (Ac.). - Meyr., I. c. (1892) (Lept.). Pront in Seit\%, 1. c. (1913) (Ac.).

annublata Stdgr., Iris, 5, p. 156 (1892) (Ac.) Transcaspien, (Samarkand); id., Cat. Lop. Pal., 1, p. 275 Zerafschan (1901) (Ac.). - Meyr., 1. c. (1892) (Lept.). - Prout in Seitz, Maclolep!, 4, p. 56, t. 41 (1913) (Ac.).

cumulata Alph., Hor. Soc. Ent. Ross., 17, p. 160, Transcaspien t. 8 , f. 69 (1882) (Ac.) (Kuldscha); id., bis Ili Rev. Russe, 4, p. 8 (1904) (Ac.). - Stdgr.' Iris, 5, 154 (1892) (Ac.); id., Cat. Lep. Pal., 1, p. 273 (1901) (Ac.). - Meyr., Tr. Ent. Soc. Lond., 1892, p. 89 (1892) (Lept.). - Prout in Seitz, Macrolep., 4, p. 56, t. $4 \mathrm{~h}$ (1913) (Ac.).

creta ria Stdgr., Iris, 5, p. 154 (1892) (Ac.) (Central-Asien).

rubellata (Ramb., Cat. Syst. Lép. Andal., t. 21, f. 3, Spanien, 1866, non binom.) Stdgr., Cat. Iep. Eur. Portugal, (ed. 2) 1, p. 146 (1871) (Ac. consanguinaria syn.?). - Gmpbg., Nova Acta Acad. Leop. Nat., $58: 4$, p. 231 (1892) (Cn.). - ? A. Fuchs in Spuler, Schmett. Eur. (E. Hofm. Großschmett., ed. 3) 2, p. 7 (1904), t. 71 a, f. 5 (1907) (Ac.). - Püng., Iris, $21: 4$, p. 296 (1909) (Ac.). - Prout in Seitz, Macrolep., 4, p. 56, t. 31 (1913) (Ac.). - Sheldon, Entom., 46, p. 332 (1913) (Ac.). - Wehrli, Iris, $41: 1$, p. 52 (1927) (Ac.). - Zerny, Eos, 3, p. 398 (1927) (Ac.). 
r u bellaria (Ramb., MS.) Bsd., Gen. et Ind. Meth., p. 222 (1840) (Ac.) (nom. nud.).

beckeraria Zapater \& Korb, An. Soc. Esp. Hist. Nat., 21, p. 145 (1892) (Ac.). - Stdgr. \& Reb., Cat. Lep. Pal., 1, p. 273 (part;i) (1901) (Ac.). - Homberg, Bull. Soc. Ent. Fr., 1907, p. 71 (part.) (1907) (Ac.;. Reb., Berge's Schmetterlingsb. (ed. 9), p. 320 (1910) (Ac.). - Mendes, Broteria (Zool.), $9: 2$, p. 105 (1910), $10: 3$, p. 174 (1912) (Ac.) (nec Led.).

consanguinaria Mendes, Broteria, 2, p. 56 (1903) (Ac.) (err. det., nec Led.). consanguinaria? Ribbe, Iris, 23, p. 303 (1912) (Ac.).

Morphologie: Püngeler, 1. c. (1909).

Biologie: Mendes, l. c. (1910). - Wehrli, l. c. (1927).

ruficolor Prout, Nov. Zool., 23, p. $27 i$ (1916) (Ac. Transvaal (Pyl.)); id. in Seitz, Macrolep., 16, p. 63 (1933).

irrulata Warr., Nov. Zool., 12, p. 391 (1905) Kapland (Sterrha). - Prout in Seitz, l. c., t. $6 \mathrm{k}$ (1933).

nepheloperas Prout, Proc. Zool. Soc. Lond., 1916, Galla, p. $150(1916)$ (Ac. $\left.\left(P_{y l}.\right)\right)$ (Somaliland); Somaliland, in Seitz, t. c., p. 64, t. $6 \mathrm{k}$ (1933).

Aegypt.

Sudan,

Cutch,

A jmir

ocellicineta Warr., Nov.Zool., 8, p.9-(1901) (Cr.). - Kenya Prout, Proc. Zool. Soc. Lond., 1916, p. 151 (Ac. (Pyl.)); id. in Seitz, 1. c., t. 5 f (1933).

anoista Prout, Nov. Zool., 22, p. 329 (1915) (Pyl.); Kamerun Seitz, 1. c., t. 5 f (1933).

sublilala Christoph, Stett. Ent. Zeit., 28, p. 236 Sūd(1867) (Ac.) (Sarepta). - Stdgr., Cat. Lep. RuBland, Eur., (ed. 2) 1, p. 152 (1871); (ed. 3) 1, p. Trans276 (1901) (Ac.). - Millière, Icon., 3, p. caucasien 271, t. 132, f. 9 (1869) (Ac.). - Gmpbg., Nova Acta Acad. Leop. Nat,, $54: 5$, p. 491 (1890) (Id. decorata var. s.). - Moyr., Tr. Ent. Soc. Lond., 1892, p. 88 (1892) (Sterrha). - A. Fuchs in Spuler, Schmett. Eur. (E. Hofm., ed. 3) 2, p. 26, ?t. 71 b, f. 28 (1904) (Ac.). - Vorbrodt \& Müll.-Rutz, Schmett. Schweiz, 2:1, p. 27 (1913) (Ac.). - Prout in Seitz, Macrolep., 4, p. 80, t. $4 \mathrm{~m}$ (1913) (Ac.). - Culot, Noct. et Géom. Eur., 3 , p. 85 , t. 13 , f. 252 (1918).

aemulata Hulst, Tr. Amer. Ent. Soc., 23, p. 303 Florida, (1896) (Lept.). - Barnes \& Mo Dunnough, ? Texas Check List Lep. Bor. Amer., p. 101 (1917). - Großbeck, Bull. Amer. Mus. Nat. Hist., 37, p. 90 (1917) (Lept.). 


\section{W. J U N K, Verlag und Antiquariat für Entomologie Berlin W. 15}

Pars 35, 37, 39: F. Bryk, Papilionidae. 1929-1930. 675 p.

36: I. Yenstetter, Heliconiidae. 1929. $136 \mathrm{p}$.

38, 40, 41, 44: H. Stiehel, Riodinidne 1930-1931. 795 p.

42: F. Bryk, Dioptidac. $1930.65 \mathrm{p}$.

43, 46, 4S: M. Fraede, Satyridae. 1931. 759 p.

47: II. II. Shepard, Hesperidae: Subfom. Pyrginae I. 1931. 144 p.

49: \%. Concle, Drepanidae. 1931. $60 \mathrm{p}$.

50: M. Gacde, Mimallonidae. 1931. $21 \mathrm{p}$.

51: H. Stichel, Brassolidie. 1932. $115 \mathrm{p}$.

52: H. Haede, Iganaidie. 1932. $39 \mathrm{p}$.

53, 00: G. Talbot, Pieridae I, I1. 1932-1934. 384 p.

54: H. Sticliel, Amathusidae [inel, Discophoridae et Hrantidae]. 1933. $171 \mathrm{p}$.

55, 56, 5S: H. Seluissler, Saturniidae I-III. 1932-1934. 484 p.

57: H. II. Sheparl, Heperidae: Subfam. Ismeninae. 1933. 55 p.

Im Druck:

59: M. Gacde, Notodontidae.

62: F. Bryk, Lymantriidae.

63: L. B. Prout, Geometridae: Subfam. Sterrhinac II.

64: G. Talbot, Pieridne 111.

65: II. Sehiissler, Saturniidae IV (ultima pars).

In Vorbereitung:

F. Bryk, Zygacnidae (exel. Zygatena speeies palaearet.), Danaididae.

A. Busck, Stenomidae.

T. A. Collier, Lasineampidae.

J. Draeseke, Lycacnidac.

I. Gredle, Pyrahididae, "Tincoidea":

1. Incurvaridae 5. Opostegidae 9. Momphidae

2. Tischeriidae 6. Tineidae 10. Elachistidae

3. Heliozlidae 7. Gelechiidae 11. Cosmopterygidae

4. Neptieulidae 8. Coleophoridae 12. Lyonetiidae

13. Cemiostomidae.

II. Schiissler, Fupterotidae, Thunmetopoeidae, Bombyeidae, Endromiidae, MLrphiclae.

H. H. Shepard, Hesperijdae III et IV.

H. Stichel, Nymplatinae, Apaturinae.

E. Strand, Noctuidae.

G. Talbot, Pieridae IV. 
Lieferung 1 von

Animalium CavernarumCafalogus

\author{
Cafalog der \\ Höhlen-Fauna \\ Cafalogue of the \\ fauna of the caves \\ Cafalogue de la \\ Herausgegeben von der \\ faune cavernicole \\ Gesellschaft für Höhlenforschung und Höhlenkunde zu Berlin. \\ Bearbeitet von

\section{Dr. B e n n o Wo if} \\ Vorstand des Haupt-Verbandes Deutscher Höhlenforscher.
}

136 Seiten in Groß-Oktay . . . . . . . . Preis RM 18.Subscriptions-Preis") für Abnehmer des ganzen Werkes " 13.50

Diese Arbeit enthält die Aufstellung der gesamten lebenden Tierwelt in Höhlen, deren Größe dem Mensehen den Zutritt ermöglieht. - Die Fauna der Micro-Cavernen (wie Maulwurfsnester ete.) und die der Erdspalten, sowie die Quellenfauna sind also nicht mitberücksichtigt. - Das Werk behandelt nicht nur die Tierwelt, die nur in Höhlen vorkommt und sich dem Höhlenleben besonders angepaßt hat, sondern die Gesamtheit derjenigen in Höhlen vorkommenden Tiere, von denen nachgewiesen ist, daß sie in einer irgendwie gearteten biologisehen Beziehung zu den Höhlen stehen. Ausgeschlossen sind nur solehe Tiere, von denen ganz offenbar ist, daß es an solcher biologisehen Beziehung fehlt.

Die Einteilung des Werkes ist so übersichtlieh getroffen, daß der Benutzer des Werkes stets sofort die von ihm gewünschte Literatur über die Höhlen und deren Fauna findet:

1. Hinweis auf die IIerkunft der Iöhlentierwelt, ihre Lebensbedingungen und ihre Anpassung an das Höhlenleben.

2. Die gesamte Höhlenfauna-Literatur (in Wcrken und Zeitschriften).

3. Die Höhlen geographisch geordnet (also nach Erdteilen, Ländern etc.) soweit ein Vorkommen lcbender Ticre in der Literatur bekannt ist. - Angabe der auf jede einzelne Höhle sich beziehenden Literatur in faunistisch-geordneter Übersicht.

4. Die Tierwelt systematisch und geographisch nach Fundorten (Höhlen) geordnet.

5. Register der Tiernamen (Synonyme besonders gekennzeiehnet).

Ich bitte daher den ausführlichen Prospekt umgehend zu verlangen.

W. Junk, Berlin W. 15, Sächsische Str. 68.

*) Der ermäßigte Subscriptions - Preis hat nur bis zum Erscheinen der zweiten Lieferung Gültigkeit.

Druck von Gustav Feller, Neubrandenburg. 